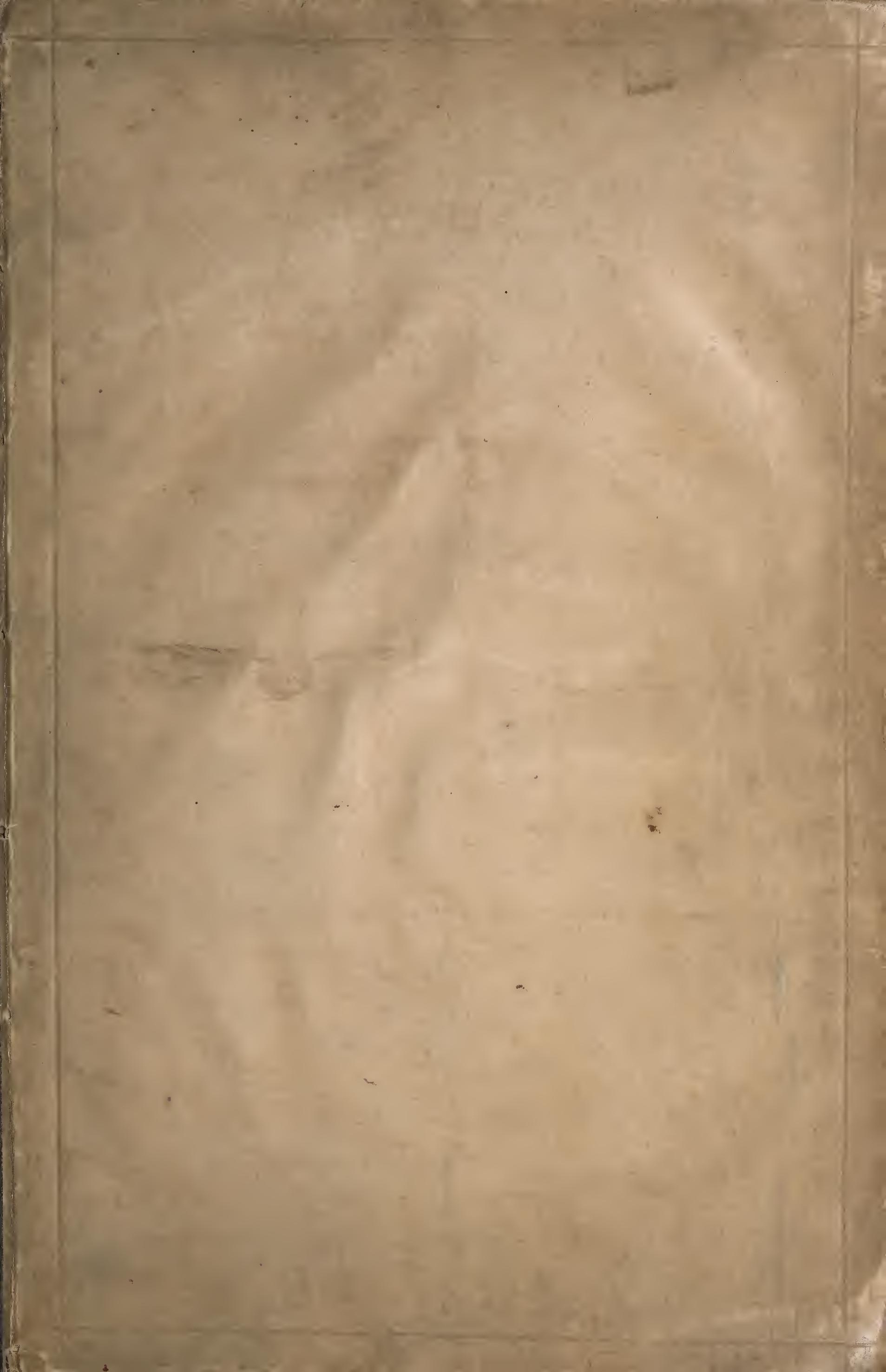






Utrecht, 2, so. 


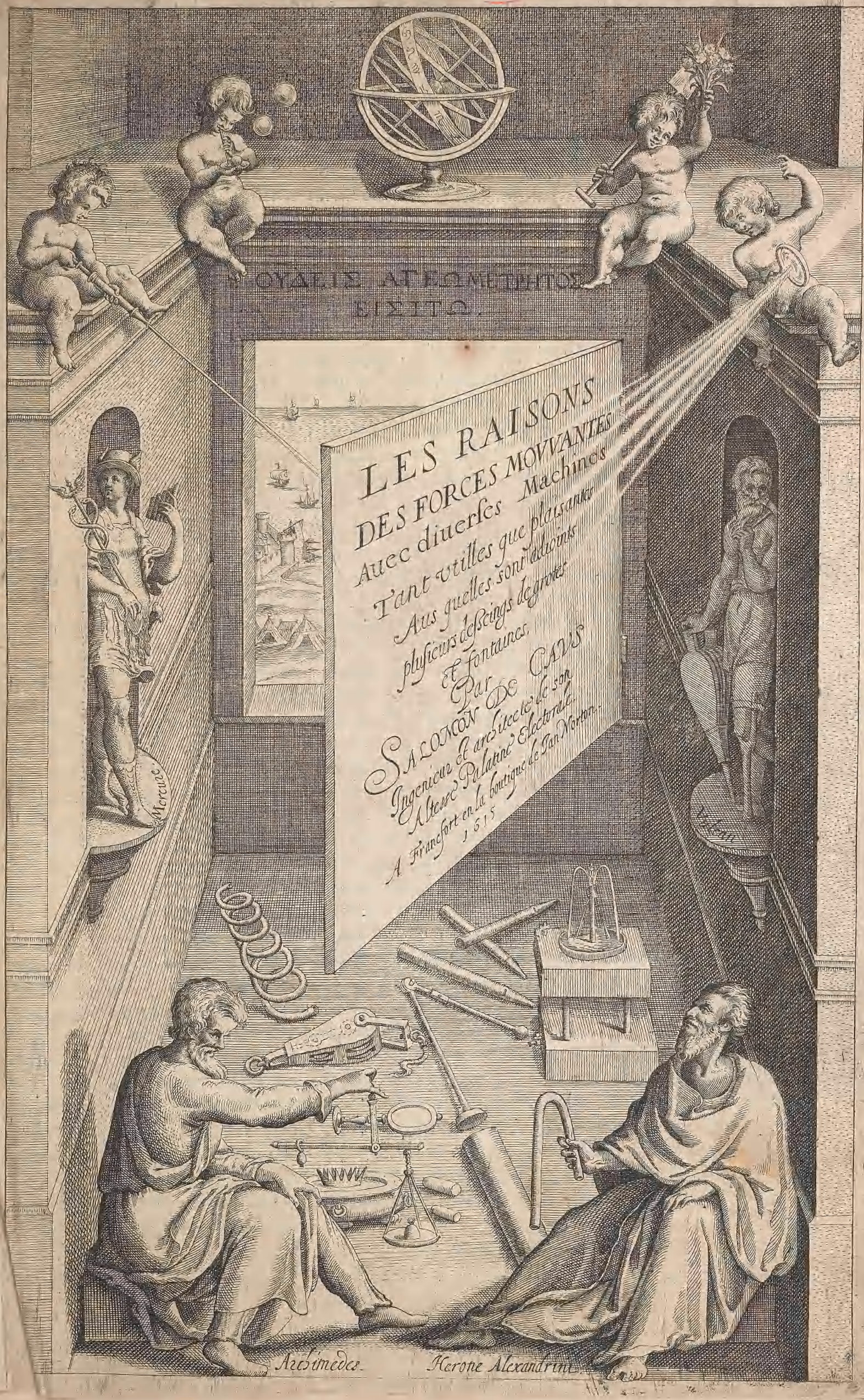




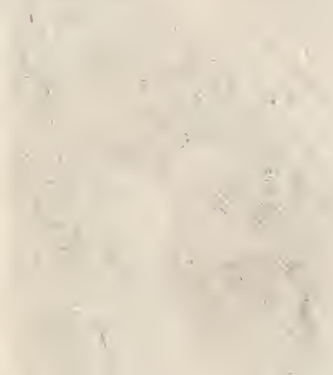

vt

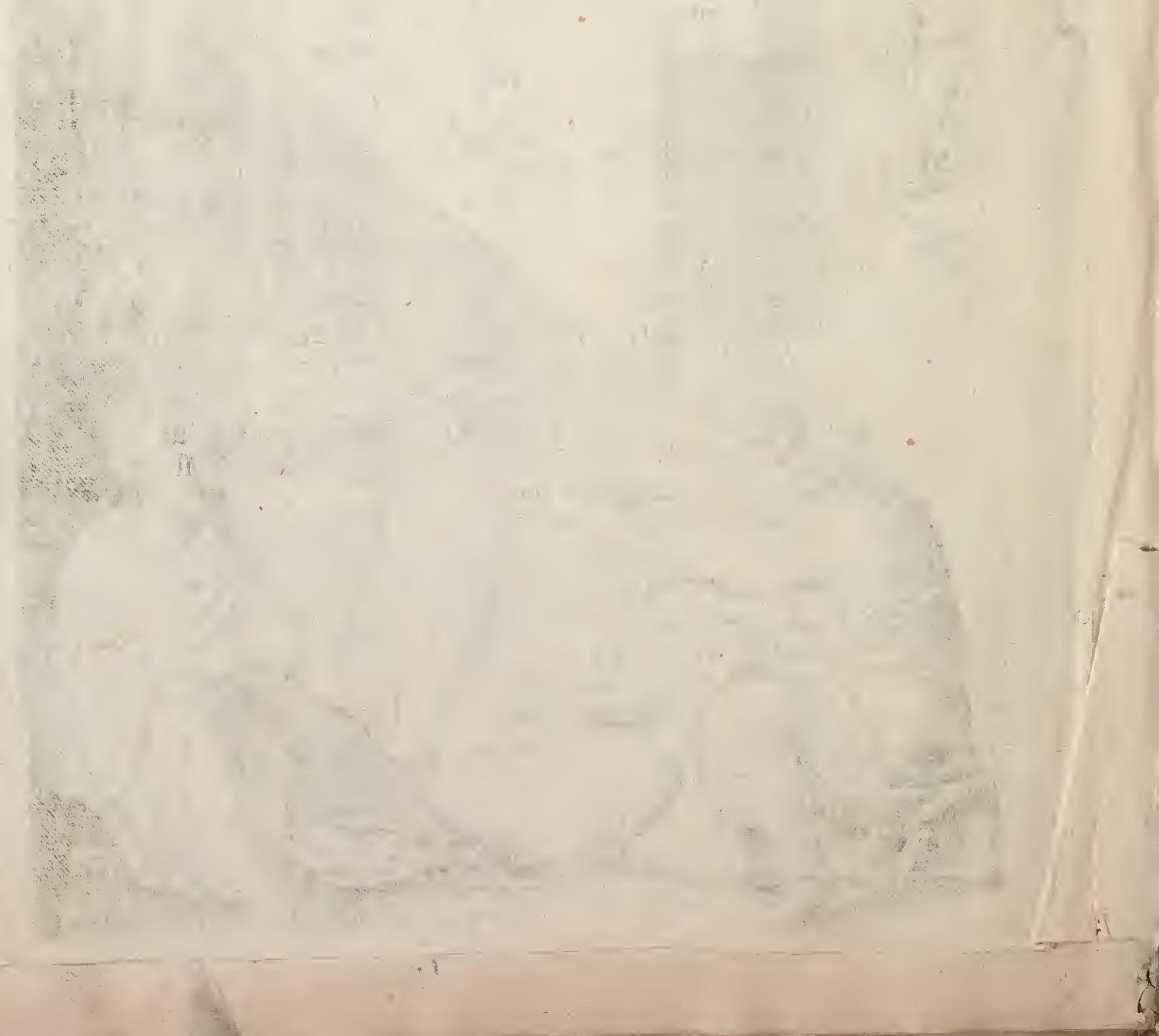




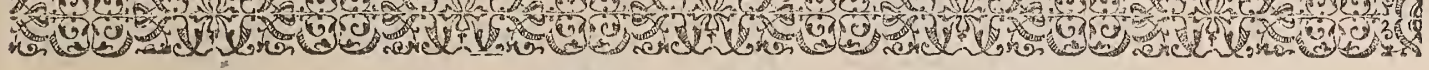
$A U R O Y$

\section{T R E S-C H R E S T I E N}

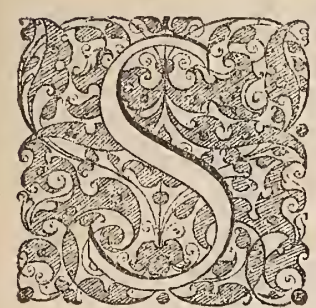

I $\mathrm{R}$ E,

A pres auoir mis fin à ce prefent liure, traitant de la raifon des forces mouuantes, \& de plufieurs machines, iay doubté fçauoir fi ledit liure meritoit d'eftre dediéà Voftre Maiefté, d'autantqu'aucuns pourront penfer, que ceft art eft pluftoft propre pour des arrifans que pour vn Roy, lequel doibe pluftolt employer fon temps à bien gouuerner fes fubiects, à craindre Dieu pour en donner vn general ex. emple, \& à fe faire redoubter à fes ennemis, cestrois chofes font tres requifes à vn Prince, carce font les trois colonnes qui fouftiennent fon Eftat, Et cn outre pour gouuerner vn fi grand nombre de peuple, il fera bon quil foit, non feulement affifté d'vn nombre de gens verf́ces en toutes fortes d'arts \& fçiences, mais auri queluy melme foir aucunement entendu, \& fpecialement aux fciences des mathematiques, \& à celles qui defpendent d'icelles, a celle fin de n'eftre fubiect a croire aucuns flateurs, lefquels voyant vn PRINCE ingnorant d'icelles, \& qu'il fe prefente ocafion de quelque ouure, luy font croire tout autrement que ladite aure ne peut reufir, tellement que cela toume quelque fois a la honte \& defplaifir durdit PR INCE, Vitruue excellent Architecte de fon temps, fait mention en l'Efpitre de fon fecond liure, adreffante à $\mathrm{l}$ Empercur Julius Cefar que l'Architecte Dinocrate de Macedone, homme doüé de belle reprefentation de corps, \& dehautes imaginations, vint trouuer Alexandre le Grand, luy reprefentant par fon difcours, qu'il auoit formé le mont Arhos en fon idée à la refemblance d'vne ftatue dhomme, laquelle pourroit tenir en fa main gauche vneville fpacieufe, \& en fa droire, vne tafle qui receuroit tous les feuues des montaignes d'a. lentour, \& dela fe defcharger dans la mer, Alexandre trouua le defeing fort beau, mais confiderant la difficulté qu'il y auroit à aporter des viores en ladite ville, eftancle païs d'alentour fort fterilen'y voulut entendre, \& du depuis fitbaftir par ledit Arcbitecte, la ville d'Alexandrie, qui eft encoresa prefent, ainfile iugement d'Alexandre furpaffa celuy de Dinocrates dautant que cegrand ouurage eut ché faic en vain, les Princes font foutent folicitez de tels Architectes \& ingenie urs (pluftof remplis de vaines imaginations que debons fondements) pourleurfire en treprendre des ouurages lefquelles ne peuuent aporter aucunevtiliténiplaifr, tellementquequand lédirs Princes font aucunement entendus, ils peuuent clairement voir par leurs deffeings que loveure ne peut eftre faite fuiuant leursimaginations, toutes ces raifons. SIRE mont donnéla hardieffedevous prefenterleditliure, ou en 7 ou 8 . fueilles, font reprefentéesles raifons des forces mou. uantes le plus briefuement \& fuccintementquil ma efté poffible, apres fuiuent quelqques machines aucunes vtilles \& d'autres plaifantes, \& entre les autres Voftre Maiefté en pourra recognoiftre quelques vnes qui peuuent eftre agirées.par le feul moyen de la temperature de l'air, lequel fe venant à elchauffer parle moy en du Soleil, ou a fe refroidir par fon defaut, anime lefdites machines, \& parce moyen l'on peut faire des chofes admirables, \& fi ie peux entendreque Voftre Maiefté prenne quelque plaifrr à ce mien petic cuure, cela me donnera courage de l'augmenter de quelques autres gentils deffeings, quil plaifedoncques à Voftre dite Maiefté le prendre en gré, atendant que iaye moyen de la feruir en chofes plus grandes, ieprie Dieu m'en fairela grace, \& a vous, SI fre maintenu en fa faincteprotection \& vous combler de fes graces, DeHeielberg ce 15. de Feburier I 6 I 5 .

De Vogfre CHaiefót le tres-obeigant fubiect.

S. de Caus, 



\section{A MONSIEVR DE CA V S}

A NACROTICHE SUR SON NOM.

$S \quad$ i les noms ont en eux quelque force ET priffance

A wecgla lageffe, lion timpofa le nom,

$L \quad$ e f̧auoir vray le rend, dont l'immortel renom,

O fte aux meilleurs eAutbeurs l'honneur des ta naiffancs:

$M$ ais fl l'efprit des morts, rentre en autre Jubftance,

$O$ u pe gliffe infenfible, auec noftre raifon,

$N$ ous penferons de voir celuy de Salomon

$D$ eslié de fon corps, faire au tien refidence

$E$ estant en ton auril,s s lage E' entendis

$C$ onioindre la fcience anec la modeftie,

$A$ yant vn eprit meur au printemps de ta vie,

$V$ ne animable douceur coniointe a la vertu,

$S \quad y$ que legrand Dieut' a, d'ornement reweftu.

I. L. M.

A U T R E,

-s y mes versefgallo yent tes excellents merites,

A nec laverité, ton beaunom glorieux,

in onverroit esclairer, dansla voufte descieux,

o utes rares vertus, (eroyent aulong defcriptes,

A ais nimonpeud'e.prit, nimefmesles charites,

- Sent touchercenom, plain de fograndrenom,

Z ayanscomprisencore, toutestesgrands merites,

$\forall$ emoyientreprend trop, voulant loüer ce nom,

is $n$ ces versmal fonnans, pour loüer Salomon,

(2) arceftronnom fcauant, außif causant eft-is,

1. yant deslong temps fait, preune deta vertu,

$\checkmark$ itrune E Archimedes E le fubtil Heron,

is onticy furpasfel du fage Salomon. 


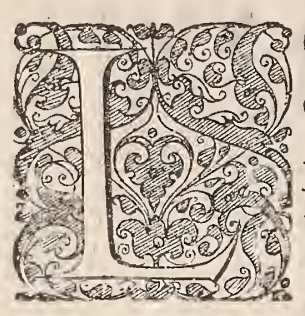

\section{OVYS par la grace de Dieu Roy de France \&} de Navarre, à nos amez \& feaulx Confeillers les gens tenant nos Cours de Parlements, Baillifs, Senefchaux, Prevofts ou leurs Lieutenans \& autres nos Jufticiers \& officiers quil aparciendra falut. Noftre bien aimé Salomon de Caus Maiftre Ingenieur eftant de prefent au fervice de noftre Cher \&z biē aiméCoufin le Prince Electeur Palatin. Nous à faict dire \& remonftrer que de Puis quelquie temps. Il fe feroir emplové en la compofition de quelques liures fcauoirl'vn Intitulé les Raifons des Forces mounanzes auec plufieurs macbines tant veilles que platfantes. Vn auec De la Theorie o pratique de Mufique. Vn autre troifieme ou font demonftés les Conftructions de quelques machines Hidranliques. Er le quatriefme contient plufieurs deffeings de grotes Artificielles \& Fontaines, tous lefdicts Liures Vtilles \& profitables au public. Mais d'autant qu'il craint que fur les Copies qu'il cn pourroit faire Imprimer, ault res Libraires \& Imprimeurs de ceftuy, noltre royaume fi pourroient Ingerer de les faire Imprimer \& mettre en vente. Le fruftrant par ce moyen de fes frais $\& x$ labeurs, nous requerant humblement nos lettres, à ce neceffa1res A CES CALS Es defirant gratifier lediet de CA us comme cftant noftre fubied, \& l'Inciter d'autant plus à continuer de profiter au public $\&$ mefmes à fin qu'il fe puife rembourcer des frais qu'il à peu faire tant pour l'Imprimerie defdiets Liures que pour les tailles doulces des Figures qui font dedans, nous luy anons Permis \& O Otroyé comme de noftre grace fpecial pleine puiflance \& auctorité Royal, luy Permettons \& Oetroyyons par ces Prefentes de faires Imprimer lefdicts Liures par tel Imprimeur que bon luy femblera \& mefmes de le faire vendre 8 diftribuer par telles perfonnes quail voudra choifir \& ce durant le terme de fix ans à compter du jour que lefdicts liures feront achevez d'imprimeur pendant lequel temps hous defendons à tous Imprimeurs \& Libraires de ceftuy noftre Royaume de Imprimer ou faire Imprimer lefdiets Liures ou aucuns d'lceulx à peine d'amande arbitraire vn tiers à nous l'autre aux pauures, \& le troifieme au denonciateur $\&$ mefmes de confifcation de tous lefdiets Liures dont ils feront trouués faifis $S$ y vous MANDons que du contenu de noftre joufte permiffion, vous laifiez jouir \& vfer plainement \& paifiblement celuy ou ceux qui aurent permiffion dudıts de $\mathrm{C} \Delta$ us fans foufrir qui leur foit faidt aucun empefchement Car tel eft noftre plaifr , donnéa Paris le dixfeptiefme jour de Uatobre l'An de grace mil fix cens quatorze \& de noftre regne le cinquiefme.

\section{Parle Roy en for Confeil}




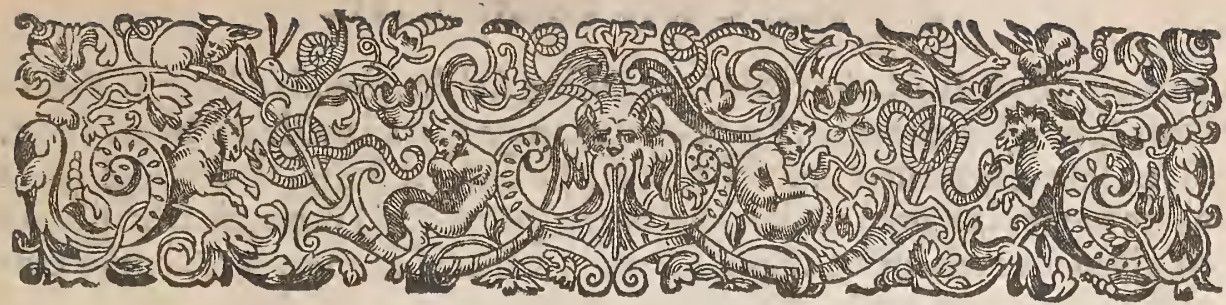

\section{E P I S T R E}

Au bening Lecteur,

\section{Oùl'Autheur monftre ce que celt que machi- ne \&lespremiersinuenteurs d'icelles,enfemblel vtilitéque lon peut tirer dece prefent Liure.}

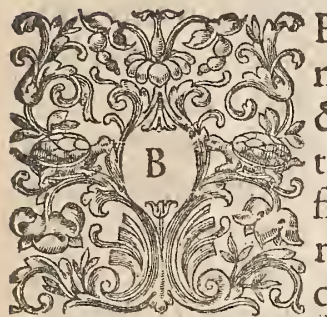

ENING LECTEUR, ayant à ce prefent liure à traiter une diuerfité de machines, il ne fera mal a propos de monftrer ce que fignifiece mor, \& les premiers inventeurs dicelles, \& auffi l'urilicé que lon en peut tirer, premierement ce mot de machine, comme dit Vitruue fignifie un' aflemblage \& ferme conionction de charpenteric, ou autre vitrexuchapo materiel, ayant force \& mouuement, foit de foymefme, où par quel. 1 . moyen que ce foit, \& y en a de trois genres: l'une appellee des Grecs Acrobactique, \& eft celle qui fert à monter toutes fortes de fardeaux en haut, dont fe feruent les Charpentiers \& Maffons, \& mefmementles Marchands, à tirer toutes fortes de marchandifés hors des Nauires, le fecond genre eft dit Pneumatique, lequel acquiert mouuement par leau \& I'air, dont il y a diuerfes machines, feruantes à la decoration de grotes \& fontaines, le troifiefme eft dit des Grecs banaufon qui fert a efleuer tirer \& porter de lieu à autre toutes fortes de fardeaux, \& mefmement a feruir de force à faire plufieurs chofes à nous dificilles fans ceft aide, comme Moulins à vent \& a eau, Pompes, preffoirs à vis, Orologes, Balances, Souflets à Forgerons, \& plufieurs autres chofes defquelles il feroit fort difficille de fe paffer, quant aux premiers inuenteurs d'icelles. Lefcriture fainte nous rend tefmoignage, que luba fur inuenceur des inftruments de Mufrque, \& Tubal-cain forgeur de tous engins de fer \& d'arain, les Payens ont creu Gene Cha. cefte invention eftre venue de Vulcan, lequel ils ont depuis adoré, comme ils ont fait pitre to tous ceux lefquels ont efté les premiers inventeurs des chofes neceffaires à l'homme, mais de ces premiers inuenteurs n'auons aucune cognoiffance d'aucune machine par eux inventee, comme de ceux qui ont fuiui depuis, entre lefquels Archimedes a laifsé plufieurs chofes par luy inuentees, comme lá vis dont fait mention Diodore Sicilien, Diodere Silequel dit qu'Egypte fut merueilleufement fecourüe contre les inondations du Nil. Par cilien en forn la vis d'Archimedes, il inuenta auffi plufieurs machines de guerre pour deffendre la byfioure arsville de Siracufe que Marcellus tenoit affe gee, commePlutarque recite, toures lefquel- premier. les machines ont efté delaiffees depuis quel'úage du Canon eft venu. Viron le tēps d'Ar- Prutarquas chimedes, eftoit (Stefibie duquel Vitruue fair mention, \& dit qu'il fut inuenreur de Marcellar. plufieurs machines dites des Grecs Preumatiques \& Hidrauliques, (ceft à dire eaux Vitrusulichantans.) Ce futluy qui inuenta de mefurer le tempsauec le cours de leau, laquelle in- pres 9 . Cha uention n'a plus efté en ufage, depuis que les Orologes à rouës dentelees ont efté in. uentees, apres luy vint un Filon Bifantin, duquel Herone Alexandrin fait mention, difant auoir efrit quelque chofe de ceft art, \& depuis ledit Filon, eft venu Herone Ale- Heroneare xandrin, lequel nous a laifsé trois liures. Sauoir un intitulé Spiritali, traitant diuers $\int_{a}$ Maching problefmes des effets de lair \& leau, \& le fecond de la machine mouuante, dite des frabilso 


\section{Epiftre au Lecteur.}

Grecs Automatij, \& le troifiefme, de la machine ftable, depuis eft venu Vitruue du" quel les cfcrits font affez cogneus. Et quelque temps apres la ville de Rome fut deftruite par les Gots, lefquels ruinerent les plus belles Oeuures d'Italie, \& des lors les peuples de l'Europe nefirent plus aucun compte des arts, jufques à la venue de trois grands Princes, lefquels vivoient tous en vn mefme temps, à fauoir l'Empereur Charles cinquierme, le Roy François premier, \& le Roy d'Angleterre Henry huitiefme, lefquels eftoient tous curieux de reftablir les arts enfevelis defi longues annees, \& un peu apres eux vint le Pape Sifte V.lequel fit reftablir à Rome plufieurs ruines, qui auoient efté faites par les Gots, \& en outre fit faire diuers ouurages rares, \& à les nombrer, un grand Volume ne fufiroit, quandaux hommes d'art rares, qui ont efté du temps de l'Empereur Charles $V$. il y en a eu plufieurs en Alemagne, entre lefquels Albert Durer, a efté recogneu vn des plus excellens de fon temps, \& auffi du mefme temps eftoient en Italie Michel l'Ange; \& Raphael d'Urbin, affez, cogneux par leurs ou ures, un peuapres font venus en. France Pierre Ramus, Oronce Finé .. \& plufieurs autres grands perfonnages, pour les Mathematiques, peinture, \& Architecture, mais pour reuenir à ceux qui ont eu cognoiffance des Machines mouuantes \& Hidrauliques, peu en ont efcrit de noftre temps, bien eft vray, que Jacob Beffon, Auguftin Ramelly, \& quelques autres ont mis en lumiere quelques Machines par eux inventees fur le papier, mais peu d'icelles peuuent auoir aucun effect, \& ont creu, que par vne multiplication de roües dentelees, lefdites machines auroient effect, felon leur penfee, \& $n^{\prime}$ ont pas confideré, que ladite multiplication eft liee auec le temps, comme il fera monfté en fon lieu : Et quand à I'vtilité que l'on peut tirer de ceft art, il eft certain qu'il eft grand, \& de nombrer la quantité \& diuerfité des machines qui font faites pour le feruice de l'homme il feroit prefque impoffible, quand à celles qui font en ce liure, les vnes font faites pour l'v tilité commune, \& les autres, pour le plaifir \& ornement des Palais \& Jardins, dont ay fait l'experiēce de la plus part, \& quand aux difcours que i'ay fait fur chacune figure, aucuns le pourroient trouver un peu trop prolixe, d'autant que ie recite quelquefois u. ne mefme chofedeux fois, cela ay-ie mieux aiméfaire, que de laiffer aucune chofe qu'el. le nefoit parfaitement entendue, car fi le Lécteur n'entend la chofe eftant propofee d'une façon, il entendra peut eftre de l'autre propofition. 


\section{Des forces moirizantes.}
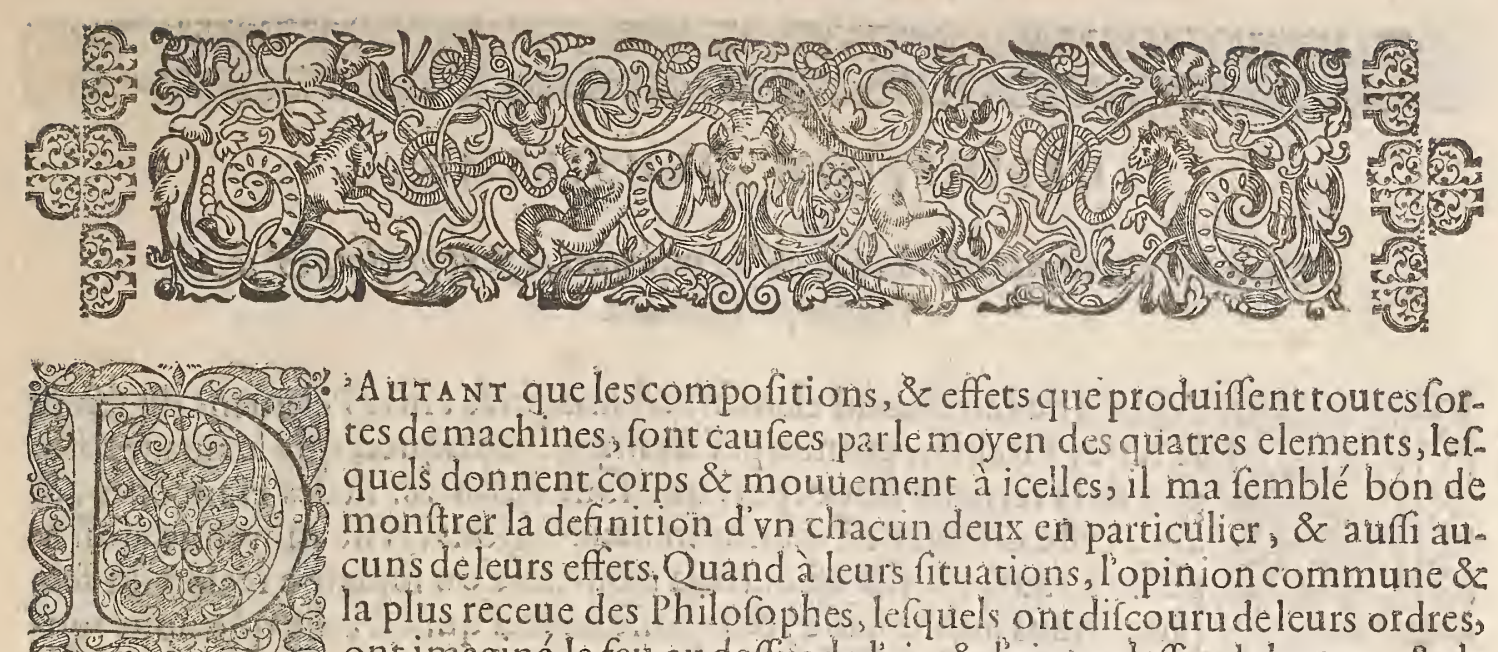

A u T A N Tue les compofitions, \& effets que produiffent toutesfortes de machines, font caufees parle moyen des quatres elements, lefquels donnent corps \& mouuement à icelles, il ma femblé bon de monftrer la definition d'vn chacun deux en particúlier, \& auffi au. cuns de leurs effers, Quand à leurs fituations, lopinion commune \& la plus receue des Philoro phes, le quels ont difcouru deleurs ordres, ont imáginé le feu au deffus de l'air, \& l'air au deffus de la terre, \& de leau, les deux premiers eftans legers voulans roufiours monter en haur, \& les deux der. niers pefans, voulans tò ufiours defcendre en bas.

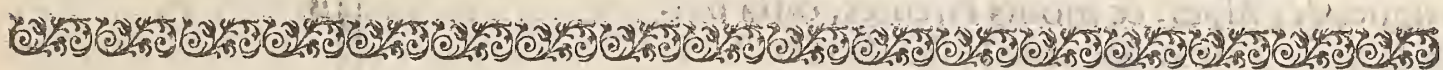

\section{DEFINITION PREMIERE.}

\section{Le Feu, eft in element lumineux, chand tres-fec Es trés-leger, lequel par fa chaleur fait grande violence.}

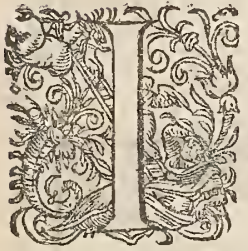

L y a de deux efpeces de feu, l,vn eflementaire,lequel n'eft fubiect à corrü. ption, lequel ie croy eft re la chaleur du Soleil, car tout autre feu ou chaleur eft fubiet à nourriture, \& $x$ ce qui eft fubiet à perir, don ques la chaleur proce. dantedu corps du Soleil, eft le feull feu elementaire, la feconde efpece de feu. Subi quand à ce qu'il eft dit lumineux , c'e ft à caufe du Soleil, qui eft la vraye lumiere naturelle, \& mefmement la lumiere artificielle procedè du feu materiel. La fecheté auffiy eft, céla re voic en ce qu'ileft directement ennemi de l'humide, \& mermes qu'il cherche à le de. ftruire, \& les chofes mefimes que nous eftimons feches, font encores a fechees parle feu, comme par exemple, cen'eft pas chofe commune, que de croire quil y aye aucune humidité au plomb, toutesfois l'experience nous monftre, que le plomb en table dequoy font coutertes les maifons \& Eglifes, fe defeche fi fort auec le temps par la chaleur du Soleil, quil fe cofine \& recire en dedans; \& fi lerdites tables font fortatachees contre le bois, \& qu'il ne fe puiffe recirer en dedans, leidites tables fe creueront en plufieurs places, le feu eftauffi dit trefleger pour plufieurs raifons, premierement à caufe de fa fituation, en ce qu'ileft au deffus desautres elemens, \& auffi que nous voyons le feu. materiel monter en haut, auec grande legereté, \& femble (comme ont dic aucuns Philofophes qưil veut retourner au lieu de fon origine, \& quand à la violence du feu, la plus grande procede du feu materiel, chacun fait le dommage qu'il fait ou il fe met, foit par accident, ou entreprife deliberee, en Sicille le feu s'eft mis dedans la concaui. té du mont Gibella, autrement dit Atrna, lequel brufle il y a fort long temps, toutesfois il y a aparence que ce feu prendra fin, quand toute la matiere fulfuree qui l'entretient finira, la violence auffi de plufieurs inuentions de machines de guerre, eft admirable, lefquelles fe font auec la poudre à canon, ainfi le feu materiel nous fert auffi bien à faire du mal, comme à faire du bien, \& quand au feu elementaire, il y a aucu. nes machines en ce liure, lefquelles ont mouuement par le moyen d'iceluy, comme l'efleuation des eaux dormantes, \& autres machines fuiuantes icelles non demonftrees par cy deuant. 


\title{
Livre premier,
}

๘\%

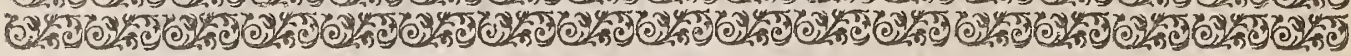

\author{
DEFINITION DEUSIESME.
}

\section{L'air, eft on element froit, fec, EO leger, lequel Je peut preffer, Ev $\int e$ rendre fort violent.}

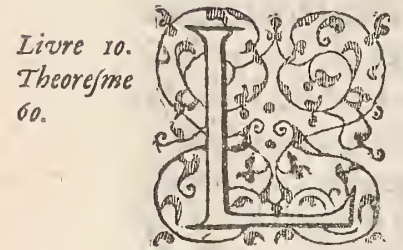

A place de l'air fecond element eft imaginee entre l'element du feu, \& la terre, Vitellion prouue par fes lingnes optiques, que le nuage $s^{2} \| l o ̈$ gne de la terre de 52000 .pas, qui font viron 26. lieües Françoifes, \& ainfi cefte diftance eft diuifee en deux regions, l'vne moyenne ainfi apellee à caufe qu'elle eft entre la troifiefme ou fupreme region du feu, (dont nous auons parlé) \& la baffe region qui eft celle que nous touchons, quand à la moyenne elle eft froide, \& remplie de nuages, \& brouillats. Lexperience nous en donne congnoiffance aux hau tes montaignes des Alpes, \& monts Pirenees, ou la neige fe maintient aucoeur de l'Efté, \& la bafferegion, comme i'ay dit, eft celle que nous touchons, ou l'air eft beaucoup plus chaud qu'aux montaignes, la raifon eft, à caufe de la reflection des rais du Soleil,lefquels donnant fur le plan de la terre, \& nepouuans paffer outre, s'areftent \& efchauffent l'air le plus bas; mais aux montaignes, les rais du Soleil ne donnent pas vne telle reflection, ains gliffent au long dicelles, \& fpeciallement aux coftees qui ne font oppofees au Mydi,ie dis donques que lair eft vn element froid, \& qui n'a autre chaleur, que celle qui luy, eft donnee du Soleil. Il n'a auffi aucun ehumidité en fa nature, cōme aucuns ont volludireaucūs, ce qui fera demonftré à la definition de leau. II eft auffi dit leger, car quelque quantité quil y aye d'air dans vn vaiffeau, il n'en fera plus pe-

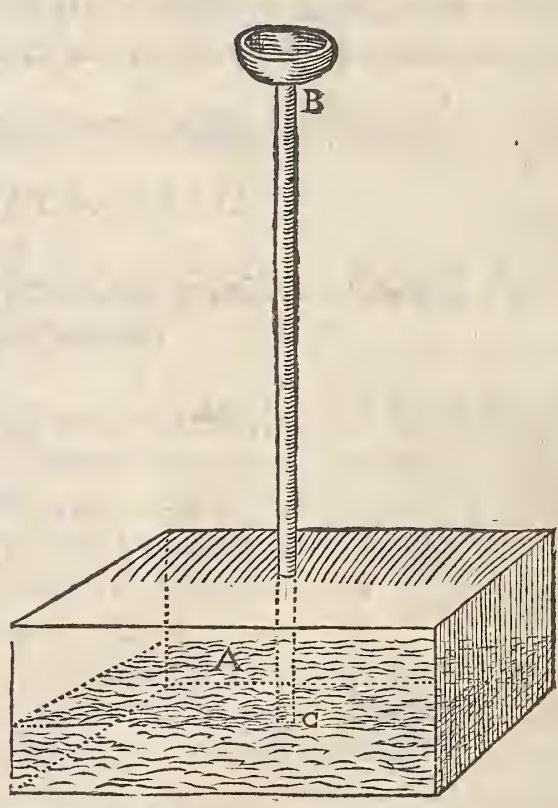
fant, \& quand à ce qu'il eft dit icy qu'il fe peut preffer, i'en donneray icy vn exemple. Soit vn Vaiffeau de plomb ou de cuivre, bien clos, \& foudé tout à lentour marqué $A$. auquel il y aura vn tuyau marqué $B$. C. duquel le bout $C$. aprochera pres du fond dudit vaiffeau viron vn pouce, \& au bout $B$. y aura vn petit recipien pour receuoir leau, laquelle verferez dedans ledit recipien, \& de là defcendra au vaiffeau, \& d'autät que l'air qui eft dedans ledit vaiffeau ne peut fortir, \& qu'il faut qu'il y aye quelque place, on ne pourra emplir ledit vaiffeau, \& fi le tuyau B. C. eft dix où douze pieds haut, il y, entrera viron iufques au tiers d'eau, tellement que l'air fe preffant, caufera $v$ ne compreffion, \& fera mefme enfler le vaiffeau, sil neft fort efpais, ce qui demonftre que l'air fe preffe, \& que cefte compreffion fait violence. Comme il fe pourra voir en diuerfes machines en ce liure, mais la violence fera grande, quand leau s'exale en air par le moyen du feu, \& que ledit air eft enclos, comme par exemple, foit vne balle de cuiure d'vn pied ou deux en diametre, \& efpaiffe d'vn pouce, laquelle fera remplie d'eau par vn petit trou,lequel fera bouché apres bien fort auec vn clou, en forte queleau ny air n'en puiffe fortir, il eft certain que fi lon met ladite balle fur vn grand feu, en force qu'elle deuienne fort chaude, quil fe fera vne compreffion fi violente, que la balle creuera en pieces, auec bruit femblable à vn perart. 


\section{Des forces mounuantes.}

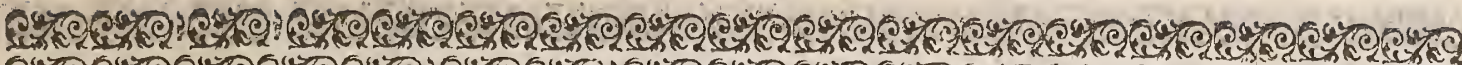
Ex

\section{DEFINITION TROISIESME.}

\section{Leak, eff un element bumide, pefant ES coulant, lequel ne fe peut preffer estant enferré.}

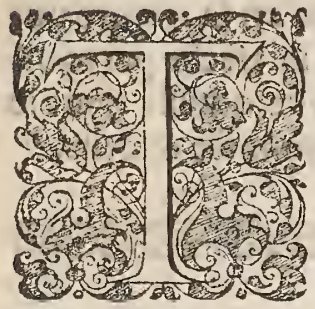

Outes lesparties de ceft element, font directement contraires à la nature du feu, l'humidité eft en leau,comme la chaleurau feu, \& de ces deux parties contraires, fe peut faire des machines admirables, commeil en fera par cy apres aucunes monftrees. L'eau eft aufi dite pefante, mais toutesfois toutes les eaux ne font de pareil pois: Pline recitant la nature de plufieurs eaux differentes, dit quau territoire de Carra en Efpaigne, il y a deux fontaines proches l'vne de l'autre, defquelles leurs natures font tant diuerfes,quetout ce qui eft misfau deffus de l'vne va 103. à fond,\& lautre porte tout ce que lon met deffus, comme auffi fait le Lac de Sodome, \& le fleuue Aretufe, la raifon de cecy vient de la pefanteur de leau, laquelle eftant plus pefante en comparaifon quele corps que lon mer dedans, le reiette en haut,à caufe que la plus grande pefanteur veut tenir le plus bas lieu, comme nous voyons par exemple, que le fer \& le plomb flotent au deffus du vif argent, car le vif argent eftant le pluspefant en efgualite de corps, veut tenir le plus bas lieu, \& au contraire, leau la plus legere ne peut fuporter aucune chofe de pefant. Pline recite encore pour chofe efmerueillable, que la pierre de ponce nage fur leau eftant en grande piece, mais eftant mife en poudre va tout à fond, la caufe en eft aifee à donner, car la pierre de ponce eftant de nature po. reuffe, \& remplie d'air, ne peut aller à fond, à caufe que l'air enclos dedans lefdites pores, veut tenir le hautlieu,comme fa nature le porte, mais eftant ladite pierre en poudre, \& quill ny a plus d'air meflé auec pourla porter, elle ira au fond, le mefime eft en plufieurs autres chofes, les pieces de bois auffi flotent fur leau, lefquelles eftans foyees en poudre vont au fond, incontinent que la foyeure ou poudre eft abreuee d'eau, par fem. blable raifon auffi, les grandes Nauires chargees de plomb, \& autres pefantes Marchandifes, font fupportees fur leau, à caufe que l'air eftant dans la concauité defdites $\mathrm{Na}$ vires, les emperche d'aller à fond, quand à ce qu'il eft dit, que ceft vn element coulant, cela fe trouue affez congneu par experience, refte à monftrer comme il ne fe peut preffer comme fait le feu \& l'air, \& en donneray vn exemple. Soit vn vaiffeau de cuiure röd, contenane trois ou quatres mefures d'eau, auquel y aura vn petit trou, pour emplír ledit vaiffeau, \& apres fi lon aiufte le bout d'vne Seringue au trou dudit vaiffeau, \& que lon voulutpouffer leau deladite Seringue dedans ledit vaiffeau, on trouuera par effect, qu'il ne fera polfible de faire entrer dauantage d'eau, que ce qui y eft entré volontairement, leau donques nefe pourra preffer pour faire aucune violence, comme fait lair où le feu, mais la violence de leau, confifte en fa pefanteur, quand elle defend des lieux hauts, ou bien quand elle eft efmeüe par le vent, on ne peut point dire cercainement, fi leau eft chaude, participante de la nature du feu, ou fi elle eft froide, participante de la nature de l'air,mon opinion eft, qu'elle n'eft ny chaude ny froide, de fa nature, mais eftant aidee du feu, ou de l'eau, reçoit par accident la chaleur ou la froidure.

$$
\text { B } 2 \text { DEFI- }
$$




\section{Livre premier,}

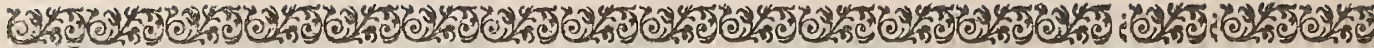

DEFINITION QUATRIESME.

\section{La terre, est un element Jec, pefant E Jolides.}

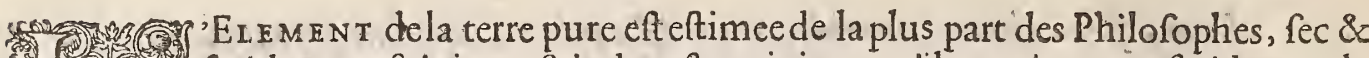
- 3 , froid,toutesfois ie ne fais decefteopinion, quily aye aucune froidure en la (2) d. Colle qui luy eft preftee du Soleil,ceft element nefe trouue en fa nature pur a commelesautres, car nous ne pouuons dire, que la terre foit feche par tout, d’autant qu'elle eft meflee de lhumidité, fa pefanteur fe monftre, en ce qu'elle tient le plus bas lieu des elemens,\& fa folidité en la maffe ronde \& ferme,compofee d’icelle.

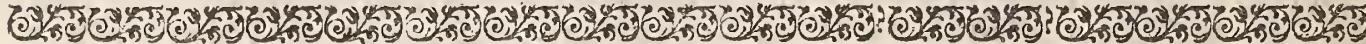

\section{T HEORES ME PREMIER.}

\section{Les parties des elemens femeslent en femble, pour vn temps, puis chacunre- tourze en fon lieu.}

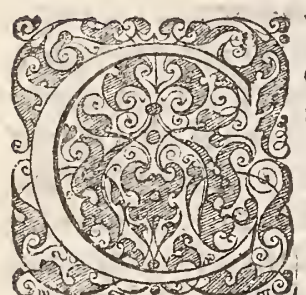

Es t chofe affeż cogneüe, que tout ce qui a efté cree par la prouidence diuine, eft compofee \& mixtionnee des elemens, com meauffitoutes lesfabriques \& compofitions que l' homme peut effectuer,com. me par exemple,le bois \& toute autre chofe que laterre procree, font mixtionnees du fec, \& del'humide, \& mefmement font deuenus

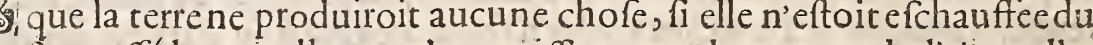
Solerl, \& ce qui elt pouffé hors icelle prend accroiffance par le moyen de l'aèr, tellement que la nature ayant donné croiffance à quelque chofe que ce foit, parle moyen des elemens, vient apres àfe deftruire par le moyen d'iceux, chacun element retournäten fonlieu, comme par exemple, le bois fe deftruit par le moyen dela chaleur, l'humidité sefuapore en haut,par extraction que fait la chale ur.Laquelle vápeur venant à monter auec la chaleur,iufquesà la moyenne region, fe qquittent l'vn lau. tre, puischacun retourne en fon lieu, l' humidi. té retombant fur la terre, qui eft-ce que nous apellons pluye, \& fur ce fubiect ie reprefenteray icy yn exemple. Soir vn vaiffeau de cuiure rond marqué A. bien clos \& foudé tout à lentour,

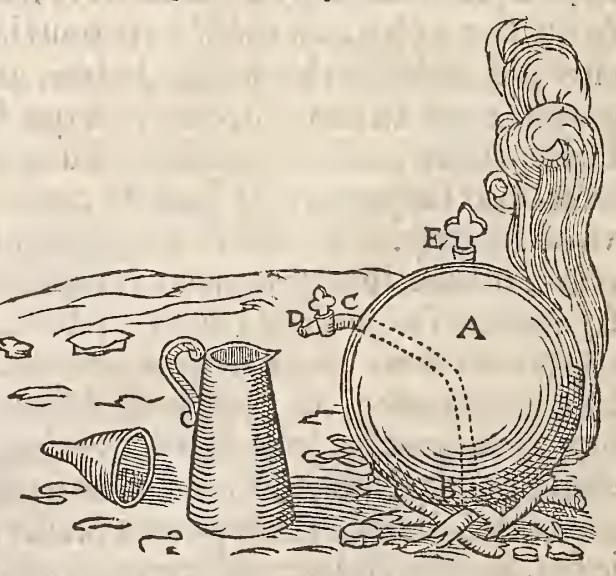
auquelily aura vn tuyau marqué $B$. C. dont l'vn des bouts $B$. aprochera du fond, autant qu'il faut pour laiffer pafferl'eau, \& lautre bout $C$. fortira dehors le vaiffeau,auquel il y aura vn robinet marqué $D$. pour ouurir \& fermer quand befoing fera, \& y aura auffi vn foufpiral en haut marqué $E$. apres faut mettre de l'eau dans ledit vaiffeau par le fouf. piral, iufques à vne certaine quantité, \& file vaiffeau contient trois pots, lony en metrra iuftement vn pot, apres faudra mettreledit vaiffeau furle feu viron 3.04 , minutes, \& laifferle fou fpiral ouuert, puis retirer ledit vaifeau du feu, \& v n peu apres faudra retirer l'eau dehors par le foúpiral, \& trouuerez que partie de ladite eau, s'eft efuaporee par la chaleurdu feu, apres faudra remplir la melure du pot commeil eftoic auparauant, \&reme. tre l'eau dedans le vaiffeau, \& alors faudra bien boucher le foufpiral \& le robinet, \& remetre le vaiffeau fur le feu,auffilong temps comme la premiere fois, puis le retirer, \& le laifer refroidir de foymefime, fans ouurir le foufpiral, \& apres qu'il fera bien refroidi, 
faudra retirerl'eaúde dedans, \& y trouuerez iuftement la mefme quantité que lon y au. ra mife,tellement quili fe peut voir que l'eau s'eftoic efuaporee (la premiere fois que lon a mis le vaiffeau fur le feu ) eft retournee en eau la feconde fois que ladite vapeur a efté enferree dans le vaiffeau, \& qu'il s'eft refroidy deluy mefme, il fe pourra encores faire $v$ ne autre demonftration de cecy, c'eft apres que lon aura mis la mefure de l'eau dedans le vaiffeau, il faudrabien boucher le foufpiral \& ouurir le robinet $D$. puis metre ledit vaiffeau deffus le feu, \& metrelepot deffoubsle robinet, alors leau du vaiffeau s'eflemera par la chaleur du feu, \& forcira par le robinet D. mais ils en faudra viron la fiziefme ou huitiefmepartieque touteladite cau neforte, à caufe que la violence de la vapeur qui caufe leau de monter, eft prouenue de ladite eau, laquelle vapeur forcira apres que leau fera fortie par le robinet auec grande violence, il y a encores vn autre exemple au vifargent autrement dit mercure, quieft vn mineral coulant, lequel eftant efchauffé par le feu, s'exale tout en vapeur, \& fe mefle auec l'air pour vn temps, mais apres que ladite vapeur eft refroidie, elleretourne en fa premiere nature de vif argent, \&l'experiencele monftre, d'autant que filon met quelque vaiffelle doree dans vne chambre ou lon aura fait efuaporerdu vif argent, ladite vapeur s'atachera toute contre ladite vaiffelle, \& lon trouuera apres que ceft purvifargent, mais la vapeurdeleau eft beaucoup plus legere, aulfi elle monte comme nous auons dit,iufques en la moyenne region.

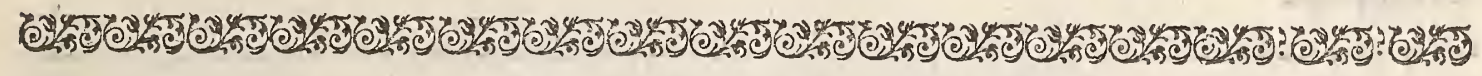
THEORESME II.

\section{Iln'y a rien à nous congneu de vuide.}

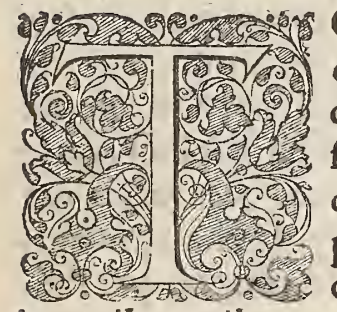

O ur cequieft congneu à l'homme eft remply des quatres elements, $c^{\prime} e f t$ pourquoy il ne peut rien auoir de vuide à nous congneu, \& de penfer(commeont voulu dire aucuns) quily a vne vacuité au deffus des elemens, ceft vne opinion fans preuue ny demonftration, \& de dire auffi, que tout ce grand efpáce eft rempli d'air, il ny a nulle aparence, car la diuine pro uidence n'a rien fait dinutile, \& filedic efpainnutile,car il ny a aucune creature qui aye à faire d'airau deftus rempli d'air, il feroit \& pour ne point errer en cefte opinion, il vaudra mieux dire que ce grand efpace eft rempli d'vne cinquiefme effence à nous incongneue. Epicure a efté vn des plus excelens Philofophes defon temps, toutefois fon opinion touchant les Atomes \& le vuide, Digegenes eft fort contraire à vn exemple que ie donneray icy, il dit qu'il n'y a rien qui ne foit corps, en la vie, \& que les Atomes font corps indiuidus \& folides, \& quily en a de deux fortes, les vnscompofez, (nous les nommons corps folides) \&les autres fimples, defquels fe fait la cópofition des cho. fes, laquelle compofition eft faite par l'acrochement des Atomes folides, (car il n'en admet point d'autres) lefquels fe viennent à tourner, virer \& entrechoquer parla caue vuidedu mödel'exem.
ple donques que ie demonftreray fera vn vaifeau de plomb ou
cuiure marqué A. clos \&bouché decouscoftez,lequel fera rem. pli d'eau par le foufpiral B. \& apres foit ledit foufpiral bien fermé,alórs fí lon fait vn petit trou au bas du vaiffeau au lieu C. il ne fortira aucune eau, dautant que vacuité ne peut eftre faite audit vaiffeau, mais fi lon donne place à l'air pour entrer audit vaiffeau, en ouurant le foufpiral B. alors Leau fortira, \& l'arr fe mettra en fa place.Orfi lopinion d'Epicure eftoit vraye, \& que la nature voudroic permettre le vuide (comme il dit quil y a entre chacun Atome) leau fortiroit, encores que le foufpiral fut fermé,d'autant quec'eft vn element pefant $\&$ coulant, ainfi nous dirons que la nature ne permet rien de vuide à nous cogneu. 


\section{Livre premier,}

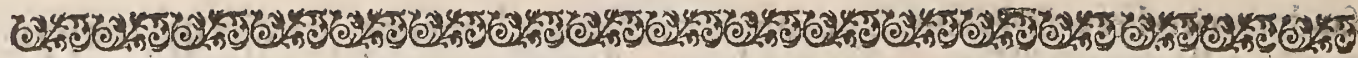

\section{AVTRE DEMONSTRATION QVE LE VVIDE ne pesst estre en la nature.}

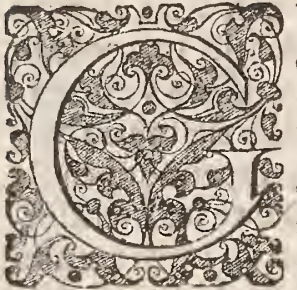

ENER ALE EMENT toutes les machines dites pneumatiques, fe fone en tant que la nature ne peut rien fouffrir de vuide, \& en donneray encores vne demonftration, ffoit vn vaiffeau marqué A. B. C. D. bien clos \& foudé de tous coftez, auquel il y aura vn tuyau, E. F. duquel l'vn des bouts $F$. aprochera du fond fans y toucher, en for. te qu'il y aye diftance, pour laiffer paffer entre ledic bout F. \& le dra boucher, \& verfer de leau dedansledit vaiffeau par le tuyau E. F. il eft certain quill y entrera quelque quantité deau dedans. Carl'air fepreffera (comme a efté monftré,) \& fe fera vne compreffion dair audit vaiffeau, de forte qu'il n'y pourra plus rien entrer, mais fi lon ouure le foufpiral, alors l'air qui eftoit en la place de leau fortira, \& à mefure que lon emplira ledit vaiffeau d'eau, l'air fortira par le foufpiral.

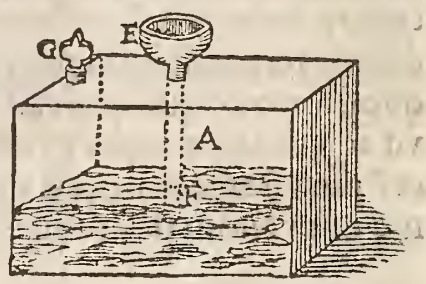

THEORES M E III.

\section{Quand leau monte par faute de vacuité, c'eft pour defcendre plus bas que fon niveau.}

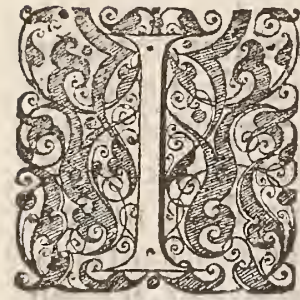

L. $₹$ A CINe moyens diuers, pour faire efleuer leau plus haut que fon niueau, \& de chacun moyen il y a plufieurs machines differentes:Le premier eft, par faute de vacuité : Le fecond, par fon propre moyen, Le troifiefme par aide du feu, Le quatriefme par l'air, \& le cinquiefme par machines compofees diuerfement, conduites par force d'hommes ou de cheuaux, ie demonftreray de chafcun mo. yen vn exemple, \& commenceray par celuy qui fait monter par faute de vacuire. Sort donques vn vaiffeau plain d'eau marqué $A$. auquel il y aura vn fifon njarqué B.C. dontl'vne desiambes fera dansle vaiffeau, \& lautre dehors.Puisfaut auoir vn tuyau marqué $D$, fait en forte qu'il fe puiffeaiufter dedanslebout C.du fifon, apresfaudraboucherle bour dudittuyau, \& lemplir d'eau,puisl'aiufterbieniufte dedās ou dehorsle bout du fifon C. puis ouurir le bout d'embas, \& alors leauforrant dudit tuy au, atire cellequi eftdans le vaiffeauau long du fifon, d'autant que vacuité ne peut eftre faiteaudittuyau, \& alors que ladite eau aura pris fon cours, lon pourra ofter le tuy au D.\&leau continuera fon cours, iufques àce qu'elle vienne au niveau du bout C. \& alors elle ceffera,ainfi lon peut voir pare eft exemple, que fi leau monte en haut par lefifon, que ceft pour de-

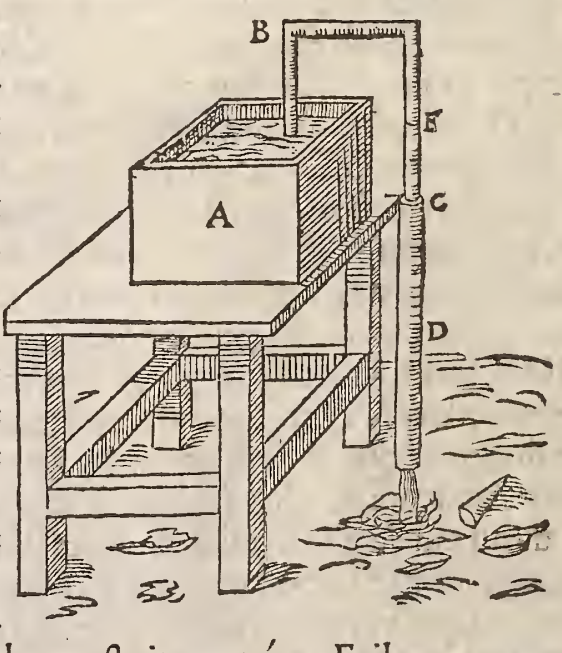
fcendre plusbas quefon niveau, carfile bout de dehors eftoit coupé en $E$. il ne courroic nullement, ainfi par laide du tuyau D.leau monte par faute de vacuité, d'autant que la pefanteur qui eft en la iambe dufifon, eft plus pefanteque celledelalambe de dedans. Et quandà la longueur du tuyau $D$. il doiteftre vn peu plus long. Que fi le fifon depuis la ruperficie de leausiufques à la marqque E. \& aufi gros queleditfifon, ou vn peu plus: 
D'autant qu'il faut que ledit tuyau D. contienne autant d'eau en longueur, ou vn peu d'avantage, comme ledit fifon contient d'air, depuis la fuperficie de leau, iufques à la marque $E$. qui eft le niueau de leau, ainfi ceft exemple demonftre, que fi lon atire l'air qui eft dedans le fifon ( foit par la bouche en afpirant, ou par le tuyau D.) leau fuiura, pour ne fouffrir vacuité , \& aura fon cours, pourueu quelle defcende plus bas que

Ce prefent THE ORESM a efté mal entendu de ceux qui ont traduit Herone, lefquels Herone $\beta$ izmonftrent à atirer leau par vn gros fifon, aiuftant vn vaiffeau au bout dudit fifon, au ritali The lieu du cuyau $D$. lequel vaiffeau ne peut faire nul effect, d'autant qu'il natirera leau orefmes. en la iambe B. du fifon B. non plus que la hauteur, comme eft ledit vaiffeau, \& encores qu'il contienne autant deau ou plus que ledit fifon, fi eft-ce que ladite eau, ne s'efleuera plus haut,que lefpeffeur ou hauteur dudit vaiffeau.

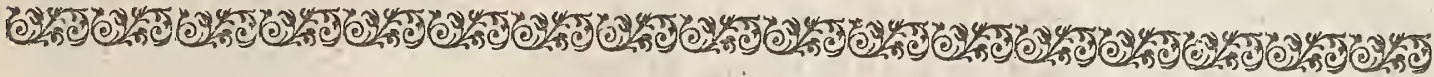

\section{T H E O R E S M E IIII.}

\section{L'eau ne pent monter par fon propre moyen; ficen'est pour defcendre plus
bas que fon niveau.}

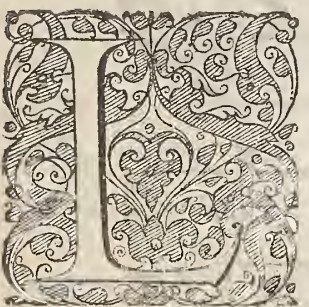

E fecond möyen de faire monter l'eau, eft parfon propre moyen, \& ce fera en ceftefaçon, foic vn vaiffeau plein d'eau marqué A. auquel. y aura vne piece de drap longue de demi pied \& large d'vn pouce, laquelle faudra mouiller toute outre, \& fera mife au vaiffeau, en forte qu'vn des bouts foit dans iceluy, \& l'autre bout dehors, alors leau qui fera au bout de dehors, attirera.par fa pefanteur celle qui eft dans le vaiffeau, \& la fera monter au long de la pieçe du drap, (comme il fe fait au fifon) iufques à ce

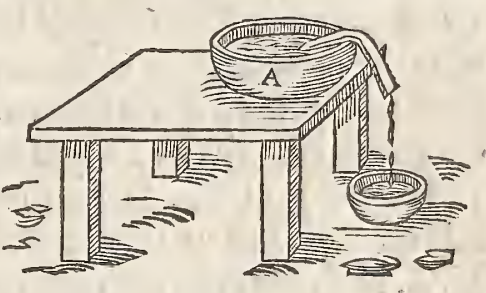
que leau du bout de dedans foit au niueau du bout de dehors, \& alors elle ceffera de courir.

2\%

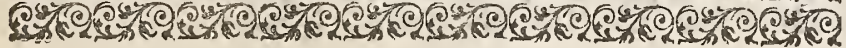

\section{THEORESME V.}

\section{L'eau montera par aide du feu, plus baut que fon niveau.}

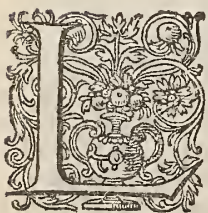

E troifiefme moyen de faire monter, eft par laide du feu, dont il fepeut faire di. uerfes machines,i'en donneray icy la demonftration d'vne.Soit vne balle de cuimarquee A. bien foudee tout à lentour,à laquelle il y aura vn foufpiral marqué D. par ou Ion mettraleau, \& auffi vn tuyau marqué B. C. qui fera foudé en haut de la balle, \& le bout C. aproche. ra pres du fond, fans y toucher, apres faut emplir ladite balle d'eau par le foufpiral, puis le bien reboucher \& le mettre fur le feu, alors la chaleur donnant contre ladite balle, fera monter toute leau, parle tuyau B. C.

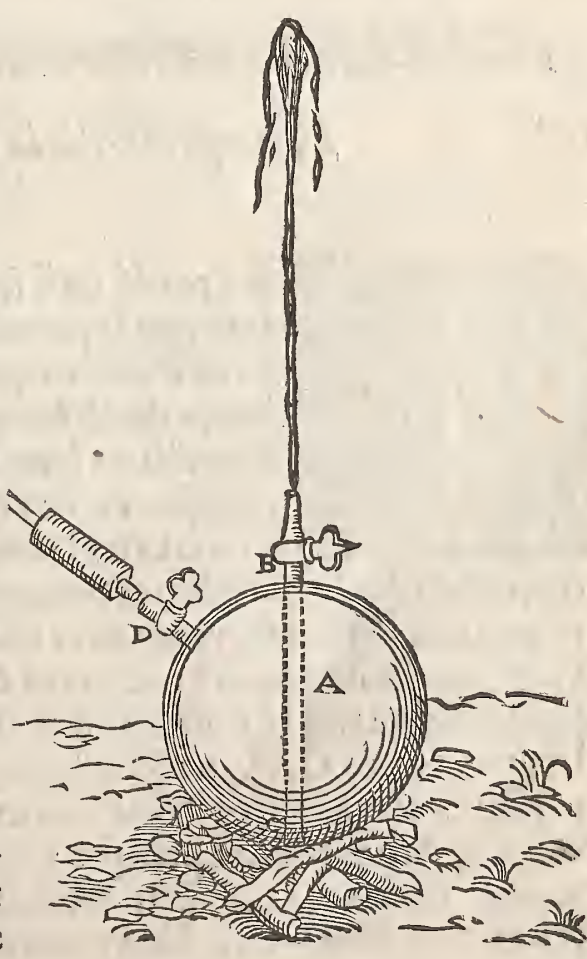




\section{Livre premier,}

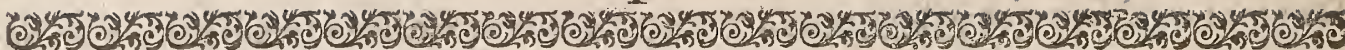

THEORES M E VI.

\section{L'eawnepeut monter par laide de lair fl ce n'est pour degcendre plus bas que fon niveau.}

Herone ßpi ritali 36 .

Theore.

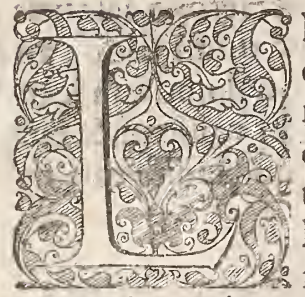

E quatriefme moyen de faire monter l'eau, eft par laide de l'air, \& en donneray auffi vn exemple parla machine de Herone, laquelle eft d'vne invention fort gentille \& fubtille. Soyent deux Vaiffeaux marquees A: \& B. bien clos, \& foudees de tous coftéz, \& pofez l'vn fur lautre, felon la diftance quelon veut faire monter l'eau, \& 3. tuyaux C.D. E.F. G.H. feront foudees aufdits vàiffeaux, en la manierequi fenfuit, trauersle cofté de haut dudit vaiffeau, \& le bout D. aprochera autant du fond du vaiffeau B. comme il faut pour laiffer paffer leau,apresfoitletuyau E F. foudéle boutE. fur le coftéde hautdu vaiffeau B. \& leboüt Faprochera autant du coftéde haut du vaiffeàu A:cồne il faut pour laiffer pàffer làir,foit lautrè tuyau G. H.foudéa trauersle coftéde haut du, vaiffeau A. en forte que le bout H. foit feulement autant diftant du fond du vaiffeau comme il eft befoing pourlaiffer paffer leau, \&y aura auffi vn foufpiral miarqué I. parlequel le vaiffeau A. fera rempli, \& apres le faudra bien boucher \& verfer de leau dans le petit recipien aù deffus du vaiffeaù $A_{\text {: }}$ làquelle eau defcendra parle tuyau C. D.àu vaiffeau dé bas; lequel eftāt ferréde tous cofteż, lair ne pourra fortir que par le tuyau

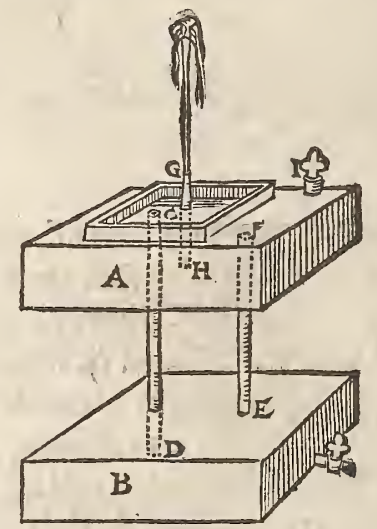
E.F. pour aler àu vaiffeau de haut, \& ne pouuantencorè fortir pouffera l'eaù parle tuyau H. G. laquelle tomberà dànsle petit recipien, \&defcendra parle tuyau C. D. \&durera ce mouuement tantqư'il y aura de leaụ dans le vaiffeau de haut.

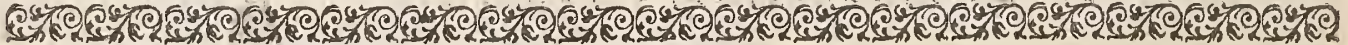

\section{Démonftration de la bauteur que la precedente machine fait monter leair.}

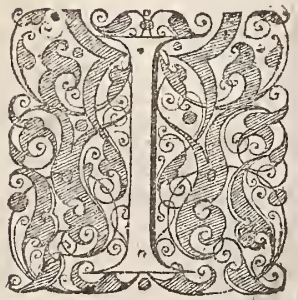

Ax penfé quill feroit bon dedemonftrer la hatuteur que la precedente machine monte fon eau, \& ce d'autant que celles quifont deffeignees aux liures de Herone \& Cardan, ne peuuent ietter leur eau en haut, quand le vaiffeau de haut eft prefque vuide, d'autant que les vaiffeaux font ioints l'vn àlautre, faris diftanceentre deux,donques quand ladite machine commencera de courir,leau defcendan. te parle tuyau C.D. fera monter celle du vaiffeau dehaut (aut tuyau G) depuis H. iufques à L. d'autant que ladite diftance eft pareille à C.D.mais quand le vaiffeau eft prefque vuide, alors là hauteur de l'eau au tuyau $C_{+}$D. nè fera fi grande, car le vaiffeau de bas eftant prefque plein acourfit ladite hauteur, de la hauteur dudit vaiffeau, $\&$ celuy de haut eftant prefque vuide, alonge la hauteur du tuyau G.ainfi rabatant l'efpeffeur des deux vaiffeaux, l'eau montera au

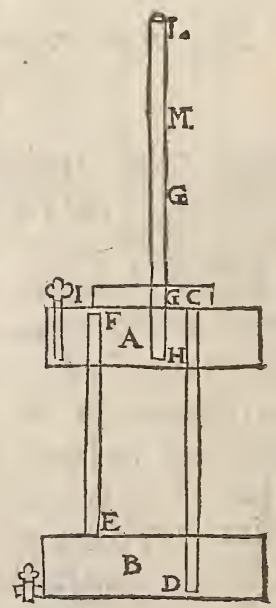
point M.quand la machine viendra à faillir. 


\section{Des forces mouuantes.}

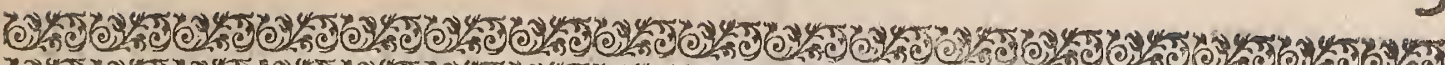

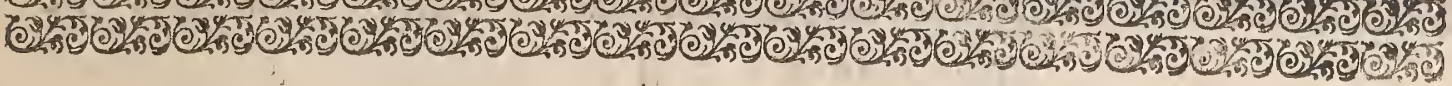

\section{THEORESME VII.}

\section{L'eau peut monter en haut par diverfes machines conduites par fa force,
mejme, ou autre que ce foit.}

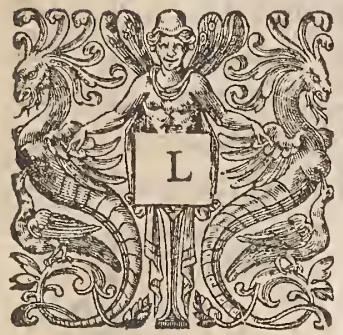

A diverfité des machines propres pour leuer leau eft grande, \& cntre toutes celles qui ont efté inventees en lantiquité illy en a vne de l'invention d'Archimedes, dequoy parle Diodore Sicilien, \& dic qu'Egypte a efté affechee par la vis d'A rchimedes, Vitruue auffi en Diodore Sse fait mention, comme auffi fait Cardan, \& dit qu'vn de Rubeis Mi calen en for lanois penfant eftre le premier inventeur de cefte Machineen devint Hiftoireanfol deioye, \&à dire vray, ceux qu'ignorent les proportions des for- premie. ces mouuantes, iugeront cefte machine eftre vn moyen de faire vn Vitruwe. li mouvement continuel, d'autant qu'jls penferont que l'eau fe haufant par ladice machine fera capable de la faire tour. ner, la fabricque en fera telle, faut auoir vn tuyeau de plomb où de cuivre, \& le tourneren façon de vis, comme la figure le demonftre, apres le faut pofer en pente comme la diagonale d'vn quarré, l'vn des bouts dedans, qui tournera fur vn pivot, \& l'autre bout fera apuiécontrevnemuraille ou piece de bois, en forte qu’il puiffe eftre tourné par

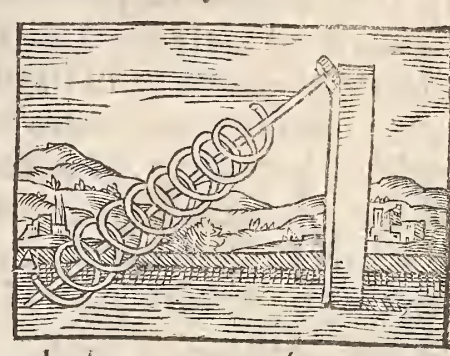
ure X.Chap. $X I$. Cardanen Safubtilite force d'homme, ou autré que ce foir, \& alors quand le bout du bas marqué A. vient à fe haufer en tournant, l'eau qui fera dans la vis, defcendra toufiours dans ledit tuyau, \& en fin fe trouuera en haut, de forte que l'eau en defcendant par ceft inftrument, monte toufiours, iufques à ce qu'elle forte, la confideration de cefte machine eft admira. ble, car la propofition d'icelle fe contredit, d'autant que l'eau defcendante par icelle monte en haut.

\section{TH E O R E S M E VIII.}

\section{Aux machines propres pour lever l'eau, la pe fanteur de ladite eau fe mefure, parfa hauteur.}

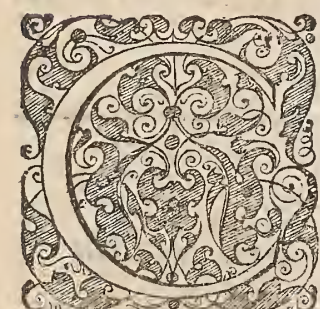

'Elt une chofe affez cogneue, que s'il y a vn trou aü fond d'vn vaiffeau plein d'eau, que l'eau fe vuidera plus vifte au commencement qua la fin, \& la raifon eft, que leau eftant de plus grande hauteur, pefe d'avantage, \& contraint celle de bas de fortir plus vifte, le mefme eft à vn tuyau qui fera au fond d'vn vaiffeau, car le vaiffeau fera bien pluftoft vuide, fi le tuyau eft long, que quand il eft court, la car fi leau ef car fi l'eau eft 24,pieds en bas, elle fera bien plus forte à tirer en haut, que celle qui n'a que I2. pieds, encores que le tuyau de I2. pieds fut beaucoup plus gros que celuy de 24. \& quil contint d'avantage d'eau, car la quantité de l'eau, ne rendra point la machine plus pefante à tirer, mais bien la longueur. 


\title{
Livre premier,
}

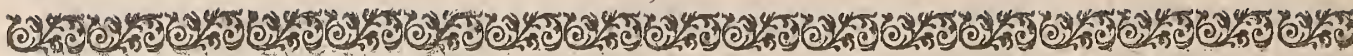

TH EORES ME IX,

\author{
Lair paffe à travers leaw quand ul est prefsé.
}

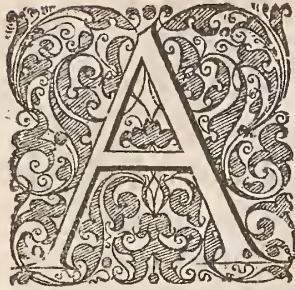

U cus s hommes fe font fort abufez en la conftruction de plufieurs machines, lefquelles apres avoir efté faites n'ont pas reuffi, ny fair l'effect ainfi quils penfoient, d'autant qu'lls ont ingnoréles raifons des forces mouuantes, ainfi ont fait ceux qui ont traduit Heron, lefquels on fait beau coup de leurs figures fauffes, \& reciteray vne fur ce fubject, pour mon ftrer que l'air paffe à travers de leâu, le cinquantecinquiefme problefme elt figuré de la façon. Soit vn vafe marqué A. B. fur la bafe L. K. M. N, auquel y aura 3. fifons comme la figure le demonftre, \& à chafcun diceux, il y aura vn petit tuyau court marqué F. G. H. lefquels feront plus gros que les fifons, en forte que leau defdits fifons puiffe paffer entre deux, ainfi verfant de leau dans le vafe A.B. quand elle viendra en la fuperficie du fifon $\mathrm{E}$. ledit fifon vuidera toure leau que l'on auoit mife dans ledit vafe, \& alors le petit tuyau $\mathrm{H}$. reftera plein d'eau, \& apres que lon remettra de leau dans le vafe, (dit le traducteur) ladite eau fe haufera iufques àla fuperficie C. fans qu'elle coure par le fifon E. d'autant dit-il que leau eftant au tuyau $\mathrm{H}$. empefchera l'air de forcir dufifon, \& par confequent d'avoir fon cours, ce qui ne peut eftre, car ledit tuyau $\mathrm{H}$. eftant court commeil eft figuré, l'air bouillonnera à trauers de leau, incontinent que leau furpaffera la

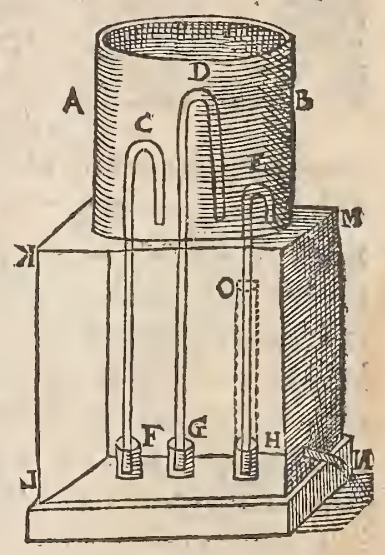
fuperficie E. de la hauteur du tuyau $H_{+} \&$ ainfi pour empefcher ceft accident, il faudroit que ledit tuyau $\mathrm{H}$. fut auffi haut, comme les lignes ocultes $O$. \& en faire aux autres cuyaux F. G. autant.Car il eft certain que leau fe mefure par fa longueur, \& fi la diftance d'entre la fuperficie du fifon, \& la fuperficie de leau du Vafe A. B. eft plus longue queles tuyaux $\mathrm{F}_{*} \mathrm{G}$. H. I'air paffera ou bouillonnera à trauersleau comme a efté dir, l'experience auffi de cecy fe voit en vn tuyau deplomb ou cuiure, carfi on met vn des bouts dans leau, pourueu quil ne foit trop profond, \& que lon foufle par lautre bout, 'air commea efté dit, bouillonnera tout a trauers de leau, il y a auffi vn pareil accident dela mefmenature quiarriue aux pompes fimples, ceft quand l'on veut forcerleau à monter plus haut, que la nature de la machine ne fouffre, l'air entrera à trauers de leau, comme fera monfté parcy apress, aux machines propres pour hauffer leau auecles pompes.

\section{THEORESME X.}

\section{La force ducontrepois qui fait mowroir sone balance, est proportionnee fuivant fon eslongnement du point de gravité.}

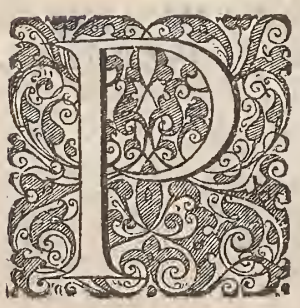

OUR donner congnoiffance des forces mouuantes par le möyen du contrepois, nous commencerons à la balánce, autrement dite Romaine, foit doncques fleau de balance marquee A. B. dont le point de gravité foit marqué $C$. \& foit ledit fleau gradué en huit parties efgualles, favoir 4 , de chacun cofté du point de grauité, ainfi fi un poids de 12 . liures eft pendu au point 1 .il fera efguallement balancé à un femblable pois pendu au point $D$. \& fi un pois 


\title{
Des forces mouuantes.
}

de 6. livires eft pendù aù point $\mathrm{E}$. il fera efguallement balancé audit pois de 12. livres pendus au. point $I$, \& fi vn pois de 4 . livres eft pendu au point $F$. il fera encores efguallement balancé aufdites I2. livres, \& fi vne des 3. livres eft pendu au point B. il fera encores efgal au dits de

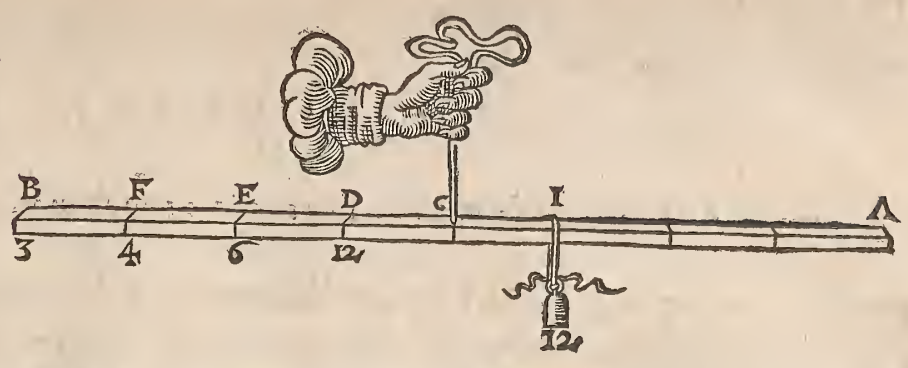
I2. livres, tellement que cefte progreffion fe fera toufiourirs en diminuant la pefanteur du pois, qui s'eflongne du point de gravité.

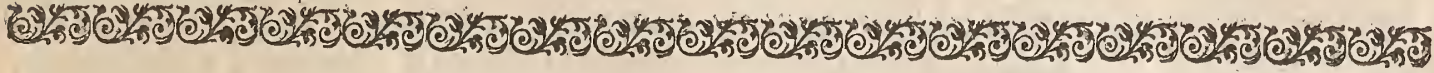

\section{THE OR E S M E XI.}

Si un des bouts de la fujdite Balance, ou Fleau eft abaiffee, tautre le leveri $E G$ toutes les. Jufdites parties mouveront, en proportion de l'eslognement du point de gravité.

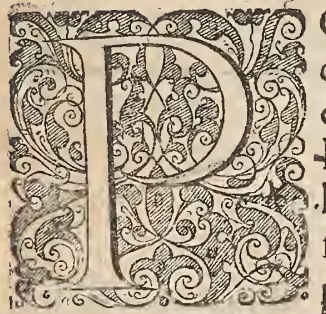

O u r demonftrer la raifon de fes proportionsicy, foit tiree uneligne droite B. A. auffilongue comme le fleau de la fufdite balance B. A. $\&$ foit le milieu de laditeligne le point de grauité marqué C.\& foit-

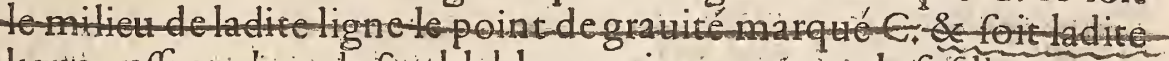
ligne auffi graduee de femblables portions comme la fuifdite, apres faut tirer vneautreligne à difcretion trauerfance le point $C$. laquelle bles portions commelautre, apres faut metrevn des pieds du compas au point C.\& de Jautre faire les portions decercles comme il fe peut voir en la figure, ainfí la portion de cercle N.D. fera efgualle à Q. I. \& O. E. fera double audit Q.1. \& P.F. Fera triple au= dit Q.1.\& M.B.fera quadruple,ainfi il fe peut voirquela proportion du poix, eft conrefpondant â la proportion dela diftance fur les portions des cercles qui font entrelefdites lignes, \& multipliant les parties des portions de cercles quifont entre lefdites lignes pàr Lu nombre du pois qui y eftioint, lon aura la quantité du premier, comme par exemple multipliant quatre parties de la portion M.B.par trois liures pefant, lon aura sz. nombre efgual a u poisdu premier point, \& ainfi fera des autres.
\end{abstract}

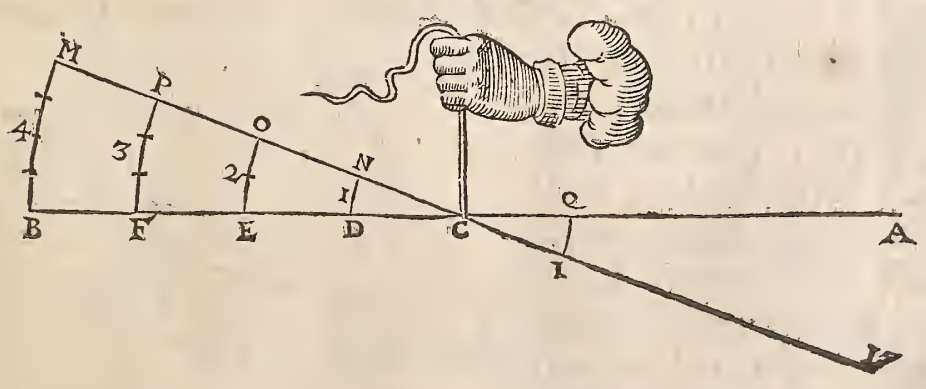




\section{Livre premier,}

r.m.

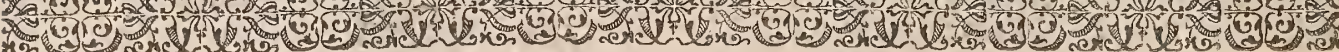

THE OR E S E. XII.

\section{Le temps de la motion saccorde avec le mouvement du contrepois.}

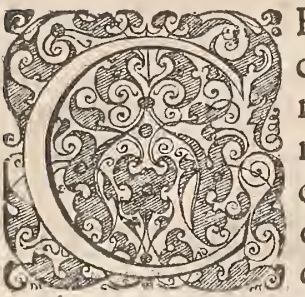

Equi eft icy apellé temps eft linterualle qui eft depuis le commen. cement du mouuement de la machine, iufques à la fin dudit mouue. ment, \& fi ceftedemonftration eftoit bien confideres, plufieurs hommes ne sabuferoient en la conftruction de diverfes machines,parlefquelles ils penfent faire efleuer vn grand fardeau par vne petite forge, ce qui eft bien poffible comme fera demonttré,mais il faut auffi - quela petite force face davantage de chemin comme a efté demonftré par la precedente, \& pàr la prefente ie demonftreray qu'il faut que cechemin fe face en mefme temps. Soit une pareille figure comme la precedente, à laquelleferaimaginé vn poids de 12 .lures au point $Q$. lequel fera abaiffer le fleau au point $I$. il eft certain que sily a vn pois de trois liures àu point B. il s'efleuera en mefme temps au point M. \& ainfi comme C.B. eft quatrefois auffilong comme Q.C. ainf B. M. fera quatrefois auffil long commeQ.I. ainfi il fe peut voir que ces deux poids eftans en equilibre

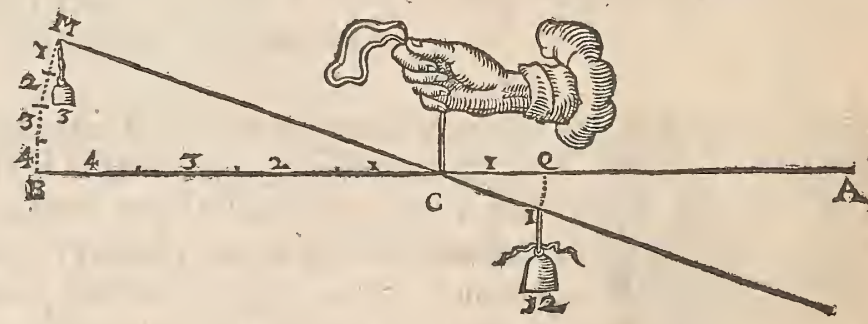
livn auec l'autre, fi l'vn eft a. baiffé, que lautre hauffera proportionnalement, felon la diftance du point, de gravité, tellement que trois liures, pourront bien leuer douze liures, vn pied de hauteur, mais il faudra que les trois liures sabaiffent au moins quatre pieds.

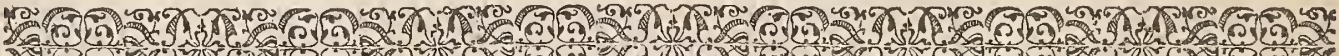

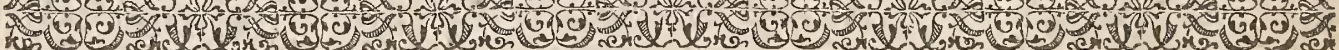
THEORES M E XIII.

\section{Le mouvement dis Levier, s'accorde avec celuy de la Balance,}

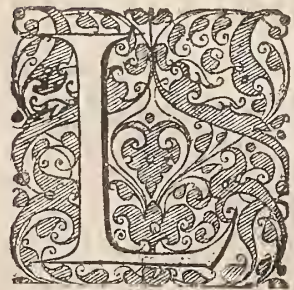

A rarson de la force du Leuier, autrement dit pied de Chevre, fe demonftreeftretelle que les precedentes, comme par exemple: Soit vne

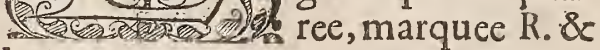
le Leuier N. O. dontla pointe O.touchera contre terre, \& apuiera la pierre au point $P$. ainfi fi la force de lhom. me leue le point C. comme fi c'eftoit cinquante liures pefant, le point P.leuera par raifon 200. liures, d'autant

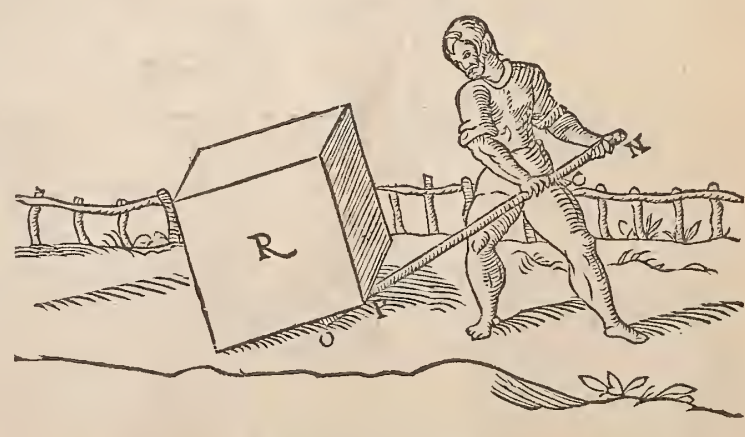
que lepoint $C$. fera quatre fois autant de chemin en mefme temps, comme le point D. aufi, il re rendra efgal, à quatre fois la pefanteur. 


\section{Des forces mouiuantes.}

T H E O R E S M E XIIHE

Aux machines qui fe font à tirer fardeauxparlemoyen despoulies, fila force esi double lons tirera 20.pieds, de corde, pour faire lever le fardeau 10.pieds.

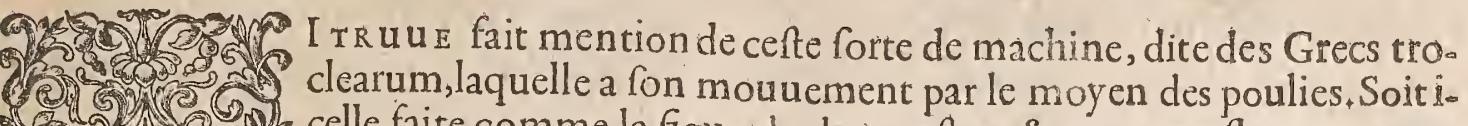
2. C $\mathrm{D}$. E. il y aura a la figure le demonftre, \& aux moufles marquees ens (n) 172 dont vn bout fera a6. (G) Saché à la moufle du haut, \& lautre bout fervira pour tirer le fardeau, comme il re peut voir en la figure, donques fi lon tire ledit bout de corde marqué $G$. vn pied en bas, le fardeau qui fera attaché à la moufle E. en mefme temps levera rn demipied, \& ce d'autant que la corde eft paffee double aux polies, ainfi fi lon tire 20 . pieds de corde, le fardeau neleuera que ro.auffi vn homme tircra auffi pefant auec cefte machine, comme en feroient deux,fila. machine eftoit fimple, mais les deux hómes tireront en mefme temps le dou. ble dela hauteurfauoir 2o. pieds, avant quelautreen aye tiréplus dedix, \& fi aux moufles 1 ly a uoit deux poulies, comme la figure M. la force feroit quadruple, mais aufin ne monteroit le fardeau que s.pieds en tirant 20 ,pieds de corde.

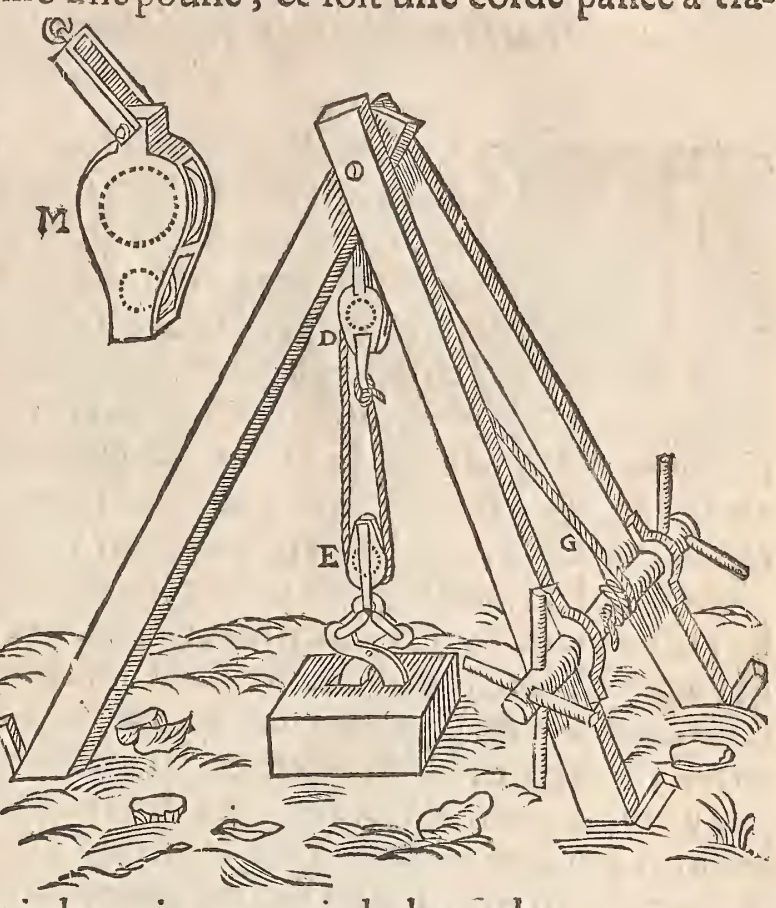

THE OR ES M E XV.

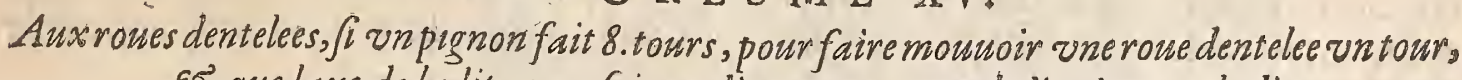
65 que laxe de ladite roue foit en diametre comme ledit pignon, ladite axe levera 8 . fois autant que ledit pignon.

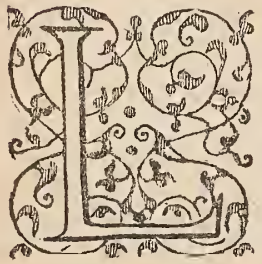

Es roues dentelees fe font encores auec la mefme raifon commeles prece. dentes, car en augmentant la force, lon augmēte proportionnalement le temps, comme par exemple, foit une machine à leuer fardeaux, faite en telle forte qu'vn pignon marqué $A$. puiffe tourner vne grande roue dentelee, marquee $B_{*}$ ledit pignonaura 6.dents, \& la grande roue 48, ainfi il faudra que ledic pignon face 8.tours cótre la grande roue vn, tellement quefi vneliure eft pendueà laxe C. elle fera efguallement balancee à 8. liures pendues à laxe E. moyennant que lefdites axes foyent de pareille groffeur, ainfi quand lon voudroit tirer 400 .

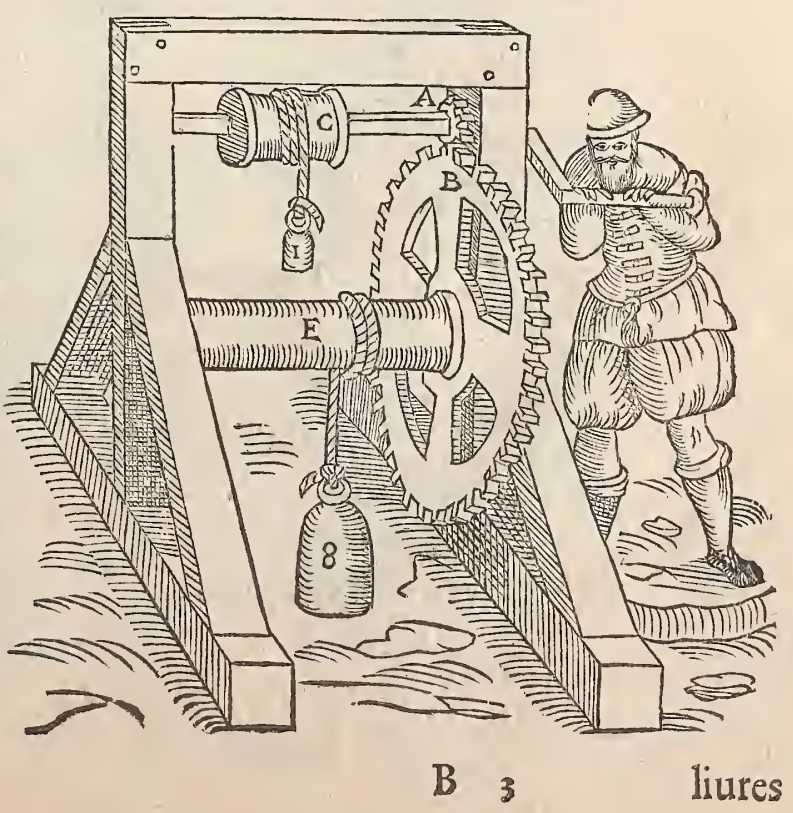




\section{Livre premier,}

liures ảuecladite axe $\mathrm{E}$. ils ne donneroyent non plus de trauail à tirer que so. liứés f $\mathrm{f}$ royentà laxe C. auffi le pois monte 8.fois autant en laxe C. comme il feroit eftant en laxe E. tellement qu'vn homme feul, fera autant de force tirant vin fardeau par cefte machine comme huit hommes feroient ayant chacun vnaxe C. mais auff fi les huif hommès font vne heure à leuer leur pois, l'tiomme feul fera huit heuresà leuer le fien.

\section{T HE OR E S M X XVI:}

\section{Parla multiplication de la force, on levera on fardeau quelquè pefant grivil foit.}

A multiplication des forces mounantes eft fi grande, quarchimedes diroit quesil eu feu ou apuyer vne mảchine, qu'il eut fait remuer la terre, veritablement la fórec des roues dentelees fe fait auec limagination iufques à linfini comme ie demonftrefay icy par vne machine encores quelle ne peur eftre mife en vfage, car il ne re prefente point de fardeaux figrands a remuer, \& mefmement on ne la pourroit faire (2) forte affez pour fuporter vn fi perant fardeau, foit donques vne rouë marquee A. laquelleaura $\$ 6$. dents, \& fera tournee par vn pignon $B$. qui aura 8. dents, ainfi ledit pignon fera $\mathbf{t}$.tours contre la roue $\lambda$. vn, äpres foit à laxe dudit pignon vne roue $C$. encores de 96 .dents mouuee par vn autre pignon $D$. auffi de 8.dents,ainfiledit pignon $\mathrm{D}$. fera auffi $\mathrm{r}$, tours cơntre la roue $\mathrm{C}$. $\mathrm{vn}$, tellement que ledit pignon $\mathrm{D}$. fera iz。 fois 12.tours, qui font 144.contre la roue A.vn touir, apres foit encores vn pareil pignion $E$. \& vine troifiefme roue F. de mefme nombre de dents, il faudra que ledit pignon E. face 1728.tóurscontrela roue A.vn,\&apres le pignon de la quarriefme roue marqué G. fera 20736. touirs contre ladite roue $\mathrm{A}_{3}$ vn, \& apres le pignon $\mathrm{H}$. fera 248832. tours, \& celiuy L. 2985984 . contre la fufdite roue A.vntour ; tellement que fi vn homme tourne tous les iours la manneuelle to ns ille tours, il ferà 298 . iours $2 \times 2$ demi pour faire tourner ladi. te roue. A. vn tour auffi fil lon metautant de liures pefant, à laxe de la roue A.comme le nombre quillfaut quela mannevelle face de tours contrela roue $A$. vin, tout ce grand fardeau fera efgualiement balancé à vne liure pendue àla manneuelle N. tellement que chacune roueque lon aiouftera, augmentera la force de $\mathrm{I}_{2}$. fois autant, \& ainfi auec cefte augmentationderoues, lon pourroit aller iufquesà l'infiny, fuituant la propofition de Archimede.

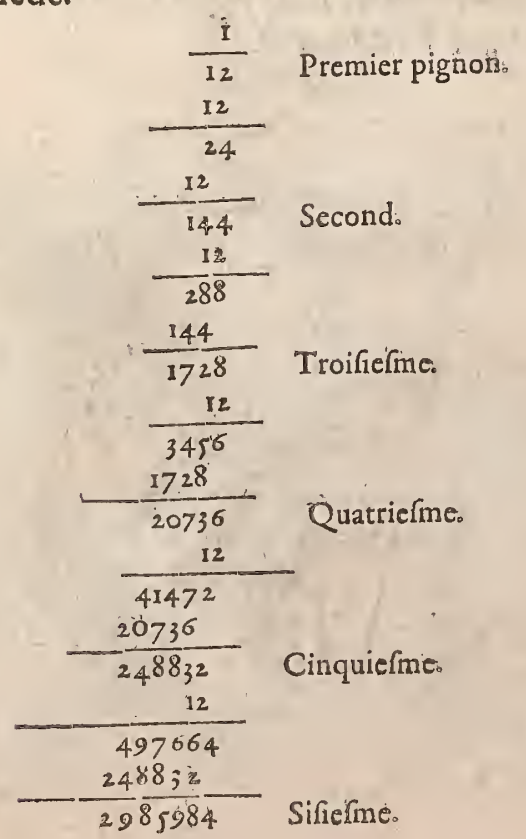




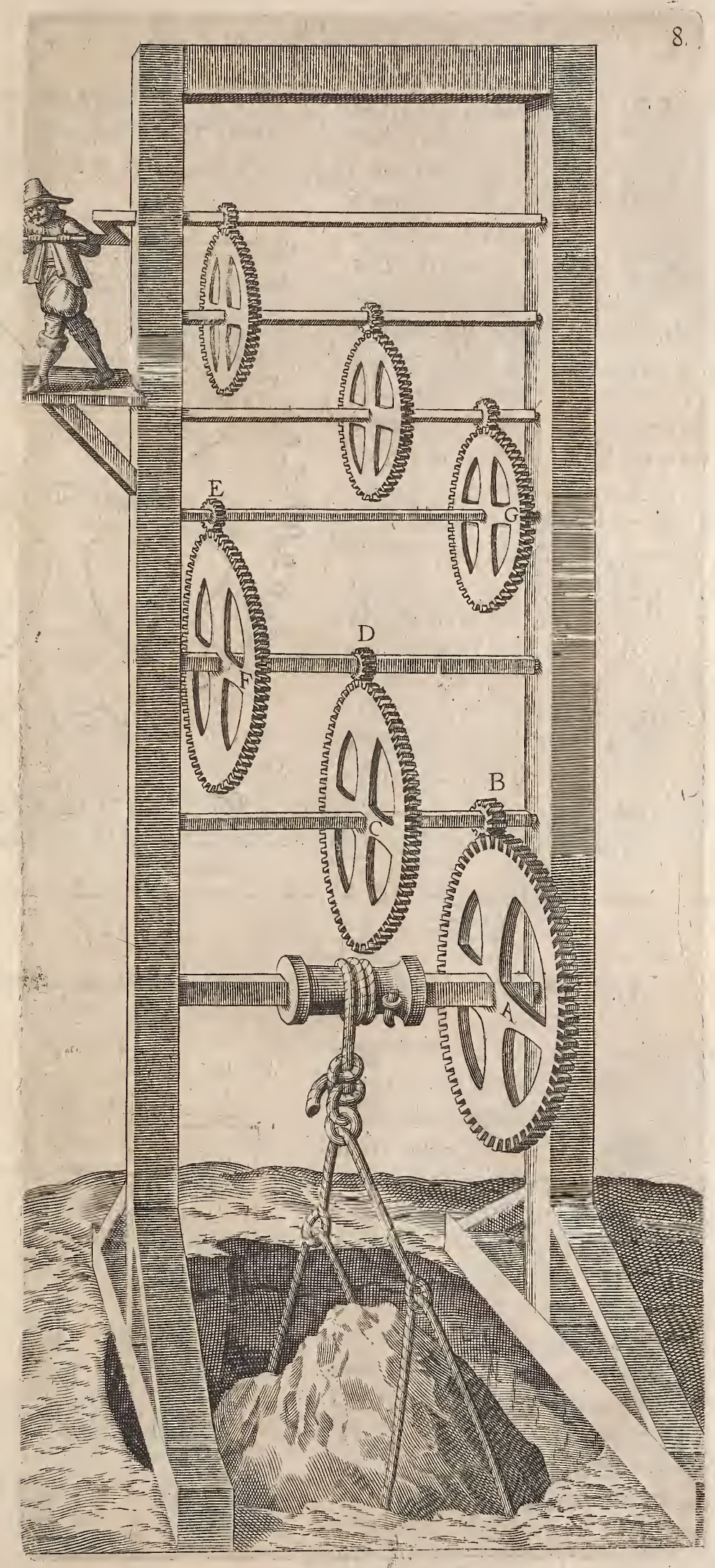




\section{Livre premier,}

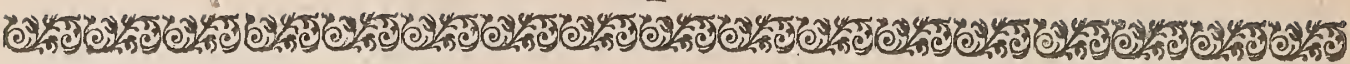

\section{THEORES M E XVII.}

\section{De la force du pignon à vis.}

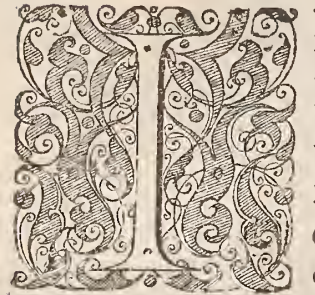

Lfe fait encores vn efpece de pignon à vis, comme il fe peut voir par la prefente figure, lequel fait multiplier la force de beaucoup d'avantage, \& auifi eft fort propre en aucunes machines, pour eftres plus tranfportables, mais il y a vne incommodité, c'eft qu'il s'ufe \& n'eft pas tant du. rable que celuy dentelé, à caufe que celuy à vis en tornant, gliffe au long des dents dela roue, \& s've fort, mais l'autre à dents en tournant pouffeles autres dents, \& ne s'vfe pas tant, \& pour demonftrer la raifon de fa force, foit vne roüe de 48 . dents comme la prochaine figure $A$. demonftre, \& foir lepignon à vis reprefenté par la lettre $B$. lequel aura l'interualle du canal de la vis, de la mefme largeur, comme les interualles des denteleures de la roüe, tellement qu'en tournant la maneuelle vn tourladite vis fera tourner la roue $A$. vnedent, \&ainfi faudra tourner ladite vis 48 .tours contre ladite roue $A$. vn tour, tellement que la force fera multipliee, commeletemps, fauoir de 48 . fois autant en laxe de la roue A. comme en laxe de la vis.

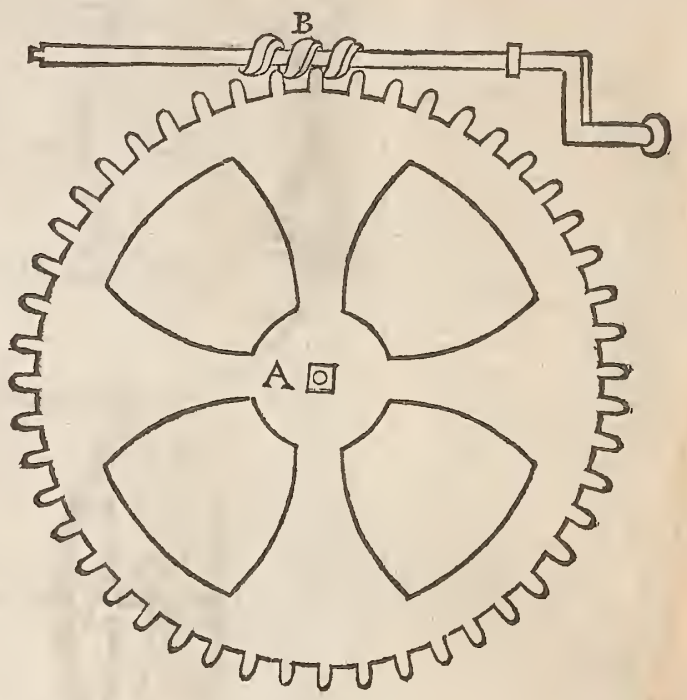

\section{La force de la preffe à vis, est conformé à toutes les precedentes.}

9201 A prefle à vis eft vne 5.5.6.

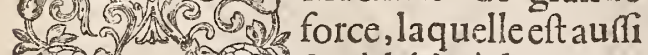
ron L femblabléà la raifon

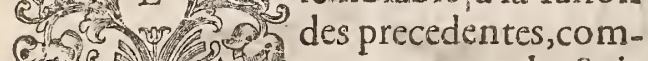

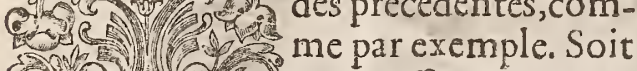
Gave) (2) $B$. laquelle aurala vis marqué $C$. $F$. dont le canal deladite vis fera vn pouce delargeur, \& foit larbre de ladite vis marqué $\mathrm{E}$. D. lequel fera 7. pieds long depuis le centre de ladite vis, iufques au point, ou l'homme eft appuyé marqué $\mathrm{D}$. ainfi fi vn homme tourne ledit arbre eflongné de 7. pieds du centre, felon la praticque d'Achimedes, il fera 22. pieds en cirćonference, qui font 264 . pouces, qu'il faudra que le bout D. face de chemin en

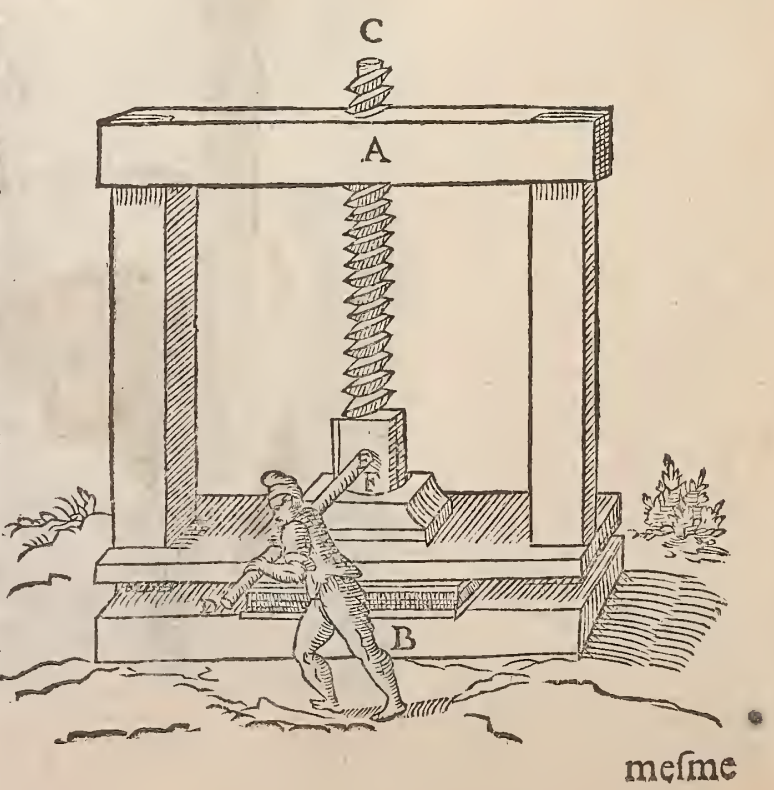




\section{Des forces mouuantes.}

mefme temps queladite vis sabaiffe vn pouce, \& partoutes les raifons precedentes, filon multiplie 264.par so.liures, quieft viron la force que l'homme aura en pouffant larbre E. D. ainfi ces nombres produiront 13200 .liures, tellement que filon met leditpois defo fus la preffe,elle aura la mefme force, commeeftant preffee auec larbre, \& force deso. liures, qui fera la mefme proportion, comme silly auoit vn leuier ou fleau graduéde 264 . pouces d'vn cofté, \& vn pouce delautre, \& quil y eut so.liures pefant, pendues au bout dudit leuier marqué $B$. il eft certain quefuiuant le trefiefme theorefine, le bout A.aura la force de leuer I 3200 .liures, qui eft la mefme force que la preffe abaiffe.

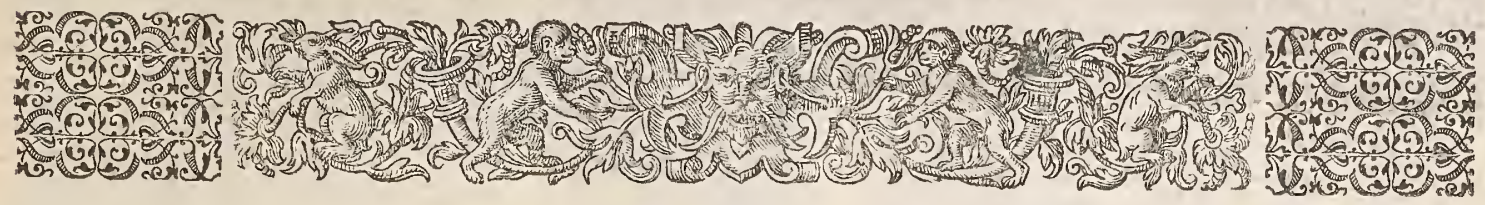

PROBLES ME PRE MIER.

Pour faire eslewer leau par le courant d'one riviere, El la force de la pompe.

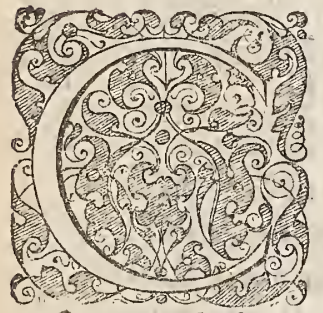
EsTE fuiuante machine vulgairement dite pompe, eft apelleede $V$ i truve \& de Herone Machine Stefibique, retenant le nom de linuen. teur dicelle, qui fut Stefibie Alexandrin, ie monftreray trois moyens pour fe feruir à efleuer l'eau par cefte machine, le premier fera par le courant d'vne riuiere (comme la fuiuante figure demonftre) ou ily a vue roue a eau, \& à chacun bout de larbre de ladite roue, il y aura vpefantur de lan maneuelle de cuiure, forte, \& baftante pour fouftenir la force \& pefanteur de ladite roue, \& fi ladite roue a dix pieds de large, $\&$ douze pieds de diametre lefdites manneuelles auront au moins quatre pouces en quarré, \& feront arrondies au millieu aux places marquees A. \& foic auffi deux pieces de bois nommees leuiers marquees par les lettres $B$. \& $C_{4}$ aiuftees dans les bras defdites manneuelles, lefquel. les quand la roue tournera, l'vn fe leuera \& l'autre s'abaiffera, \& lefdits leuiers feront aufi aiuftez dans les deux bras ou brancars marquez D.E. lefquels en hauffant feront hauffer les feaux, ou foupapes des pompes alternatiuement, \& ainfi l'eau montera dans le vaiffeau $F$. \& de là on la pourra conduire ou lon voudra, quand à la hauteur qu'elle doit monter, ie fuis d'auis quil ne la faut contraindre à monter plus de trente pieds de haut auec vne feule pompe, comme fera enfeigné au fuiuant Problefme, le gros tuyau G. eft le lieu ou la foupape eft enferree, qui fouftientleau, quandles feaux ou foupapes des pompes ne la hauffe point, la figure auecla pratique ordinaire que lon a des pompes, donnera facile intelligence de cette Machine, \& fi le courant de la riuiere eft fort, \& que lon defire auoir quantité d'eau, lon fera le diametre du dedans des bareils de dix ou douze pouces, \& que lefdits bareils ayent huit ou neuf pieds de haut, \& faut pour bien faire que les feaux haufent \& baiffent quatre pieds, \& quandils font en leur plus grande hauteur, qu'ils ayent auffi quatre pieds d'cau au deffus, àcelle fin que l'accident dont a efté parlé au neufiefme Theorefme n'aduienne, carfi leau n'eftoit haute affez entre la fuperficie du baril \& le feau, il eft certain que l'air pafferoit à trauers de leau par bouillons, \& rendroit la machine inutille, \& fpeciallement quand on la force de monter au deffus de quinze ou vingt pieds, ceft pourquoy lon prendra bien garde que ceft accident n'aduienne, la proportion auffi des tuyaux $M$. N. O.feront de quatre pouces en diametre, fi les bareils en ont douze, \& fi lefdits barils font plus petits, lefdits tuyaux feront à l'aduenant auffi plus petits, il fe fait vne autre forre de pompe appellee renuerfee de laquelle le baril eft dans leau de la riuiere, \& le feau eft 


\section{Livre premier,}

mis dedans par le bas dudit baril haufant \& baiffant en cefte façon, mais ie ne fuis d'aduis que lon fe ferue en aucune maniere de cefte inuention de pompe, à caufe des accidents qui arriuent en icelle, car l'eau montant par cefte façon, fait que beaucoup de paillettes \& ordures montent auec, \& s'arreftent en dedans les foupages, lefquelles fon empefchees de bien ferrer, \& s'il y a feulement vn poil en dedans ladite foupape,cela donnera vn grand empefchement à lefleuation de leaư,mais en cefte prefente façon cefte faute ne peut arriuer que rarement, à caufe que leau en montant en la foufpape G. s'il y a quelque ordure, elle n'y pourra monter à caufe de fa pefanteur, \& fi c'eft quelque chofe de leger il ny pourra non plus arriuer,à caufe que le bout du tuyau $O$. trempera au pied dans leau, \& ainfi ladite foufpape fera hors de danger de fe gafter par les ordures qui font meflees auec l'eau. 


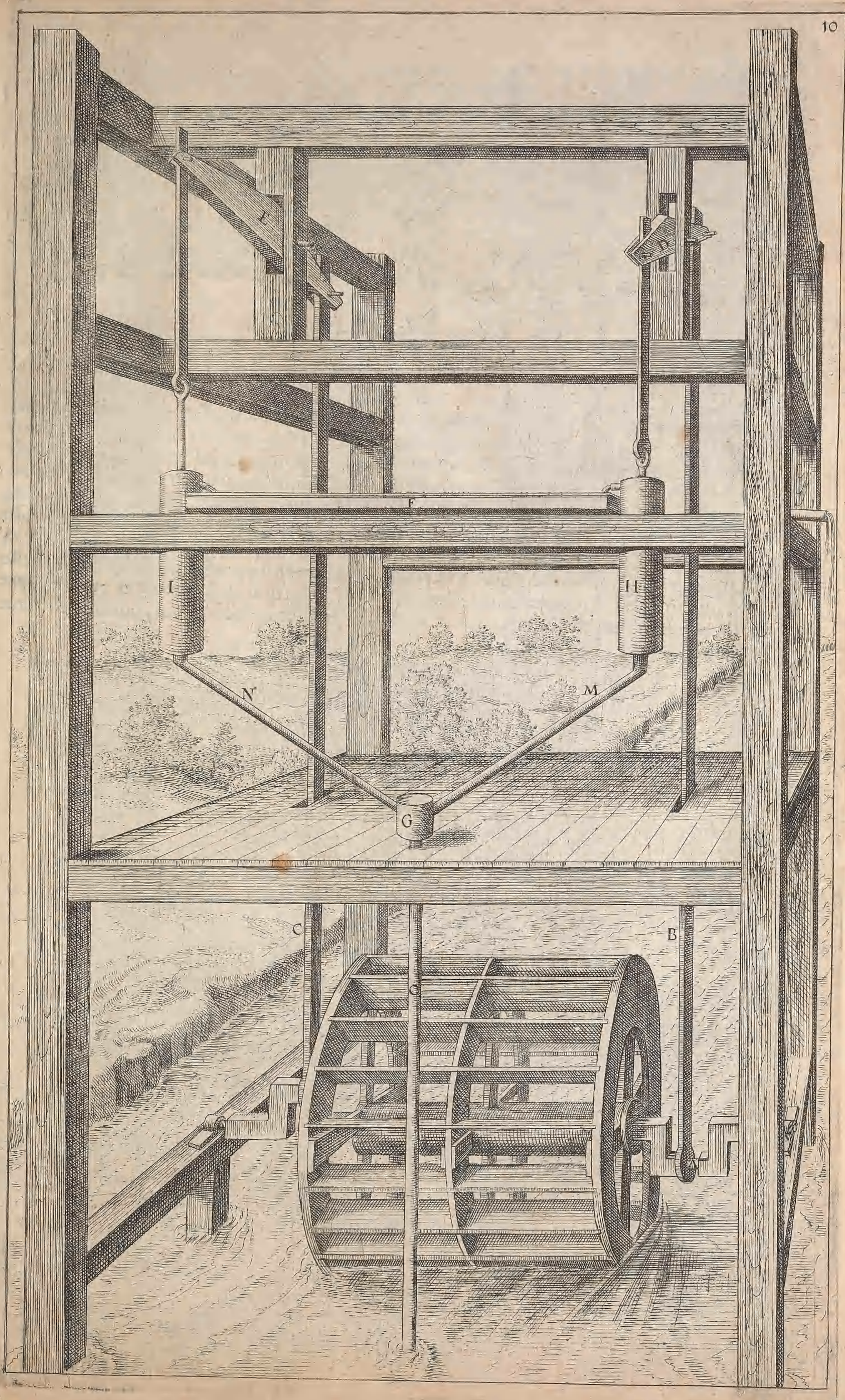




\section{Livre premier,}

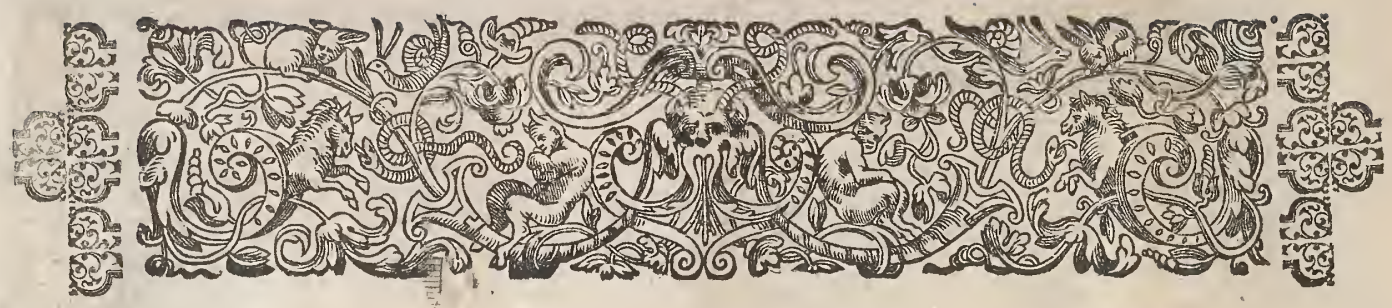

Autre moyers de lever leau, par le moyen dion ruiffeaw.

Q Este autré taçon de leuer leau, fe fera auec vn Ruiffeau d'eau efleué, ( 1 . I 2 \& quil puiffe tomber fur la roüe A. pour la faire tourner, \& en e. tournant, fera efleuer leau de la pompe B. iufques à 24 . où 3o, pieds

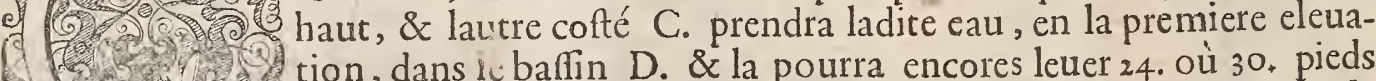
2. W tion, dans li baffin D. \& la pourra encores leuer 24 . ou 30 , pieds , haut La figure precedente, donnera le moyen \& intelligence de par le mefme moyen, comme il fe peut facilement comprendre par la figure, laquelle figure n'a peu eftre faite haute ffez, felon la proportion de fes mefures, à caufe que le papier ne la permis, mais il iera facille dimaginer ladite hauteur, comme elle doit eftre.

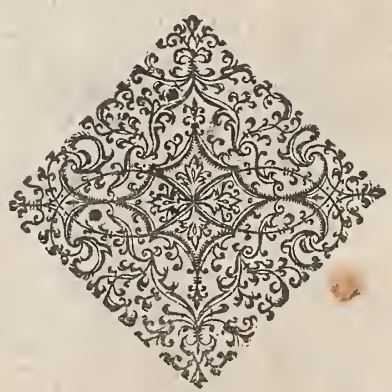




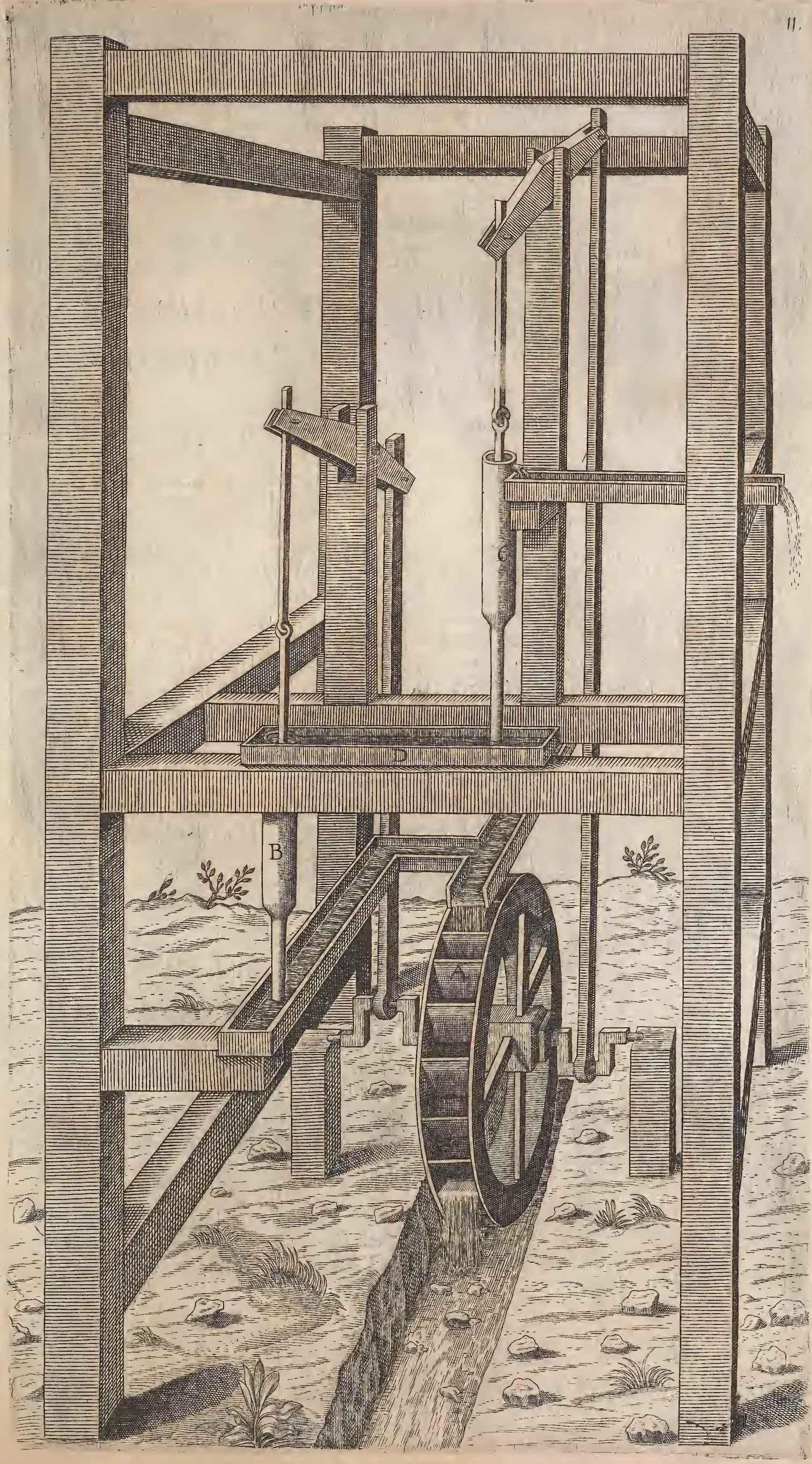




\begin{abstract}
Livre premier,

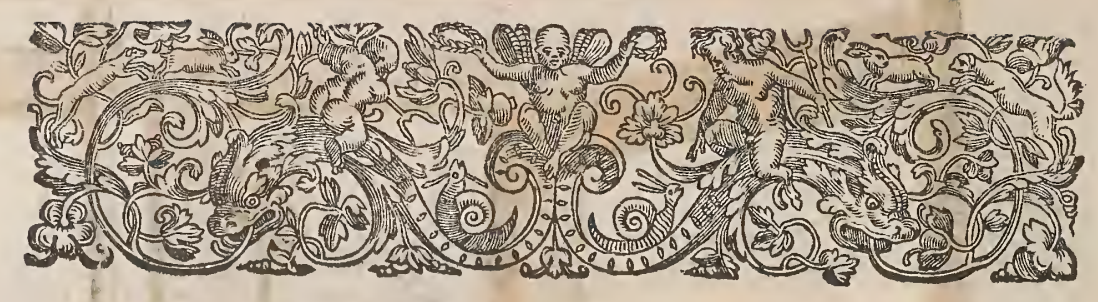

PROBLESME TROISIESME.

Pour eslever une eau de fource ou de riviere par la force des chervaux.

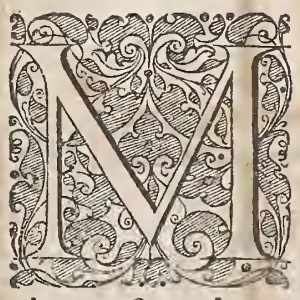

A r s s'il ny auoit riuiere affez forte, ny ruiffeau courant, lon pourra efleuer l'eau par le moyen \& force d'vn cheual, ou de plufieurs, felon la quantité $\&$ la hauteur que lon la defire, ce prefent defieing eft fait pour efleuerladite eau 60 .pides haut, \& quatrecheuaux en leueront viron 60 .muis en vne heure de temps, qui font viron 30000 .liures pefant. Soit donques premierement vn arbre de bois bien droit, vn pied . en quarré,\& 60 .pieds de haut marqué $A$. lequel tournera entredeux piuors, \&en haut pres du bout il y aura vne roüe dentelee de 24 . dents marquee B. laquelle fera tourner vne Lancerne ou pignon de 12. branches marqué C. \& audit pignon ily aura vneroüe de fer ou de cuiure, de viron deux ou trois pieds en diametre, $\&$ de dixhuit dents de tour, marquee D. mais il ny aura que neuf dents en la moitié de la circonference, lautre moitié demeurant vuide, \& y aura auff deux autres rouës, marquees E. \& F. chacune de lā grandeur de lautre, \& auffi de neuf dentsà chacune roue , \& feront toutes trois pofees les parties dentelees en haut, puis faudra pofer vne poulie au deffus, marquee $G$. ou fera paffee vne corde, laquelle auffi fera atachee ferme par les deux bouts aux arbres des roues $E$. \& F. en forte paffee,que tournant vne defdites rouës, lautre fe puiffe deftourner, comme il fe pourra voir, \& mieux confiderer en la Figure de l'Ortographie fuiuante. En apres faut bien pofer lefdites rouës E。 \& F. contre celle $D$. en forte que $D$. tournant toufiours d'vn mefme cofté, face tourner E. vn demi tour, \& alors queelle fera en la derniere dent, la premiere de la rouë $F$. fe prefentera contre la roue $\mathrm{D}$. à caufe que celle de $\mathrm{E}$. la fait deftourner par le moyen de la corde \& poulie commune $G_{0}$ \& apres que ladite rouë $D$. aura atrapé la premiere dent de $F$. continuera iufques à la neufiefme, \& apres la premiere de la roué E. fe prefentera derechef, \& ainfi les deux rouës E. \& F. tourneront, \& fe deftourneront alternatiuement vn demi tour, \& aux axes H. \& L. feront atachees deux fortes cordes, lefquelles leueront les deux Seaux qui vont dans les barils M. N. \& auront viron trois pieds de ieu, haufant \& baiffant, \& feront faits de cuiure, bien aiuftez dans les barils, \& quils puiffent iouer dedans facilement, fauoir quand ils font hauffez, qu'ils puiffent defcendre d'eux mefmes, fans eftre contrains d'eftre pouffez en bas, \& ainfi on ne mettra nul cuir àlentour defdits feaux, comme on fait ordinairement aux pompes communes; $\&$ faut noter que tantplus les feaux hauffent vifte, tant plus d'eau s'elleuera, ce qui fe peut obferuer en toutes les façons de pompes.
\end{abstract}

Faut auffi noter que les deux pieces de trauers O. P. ne doit eftre qu'vne piece, à laquelle fe doit ioindre lautre trauers Q. dans lequel trauers, tournera les quatre pi-
uots des rouës
B. C. E. F. 


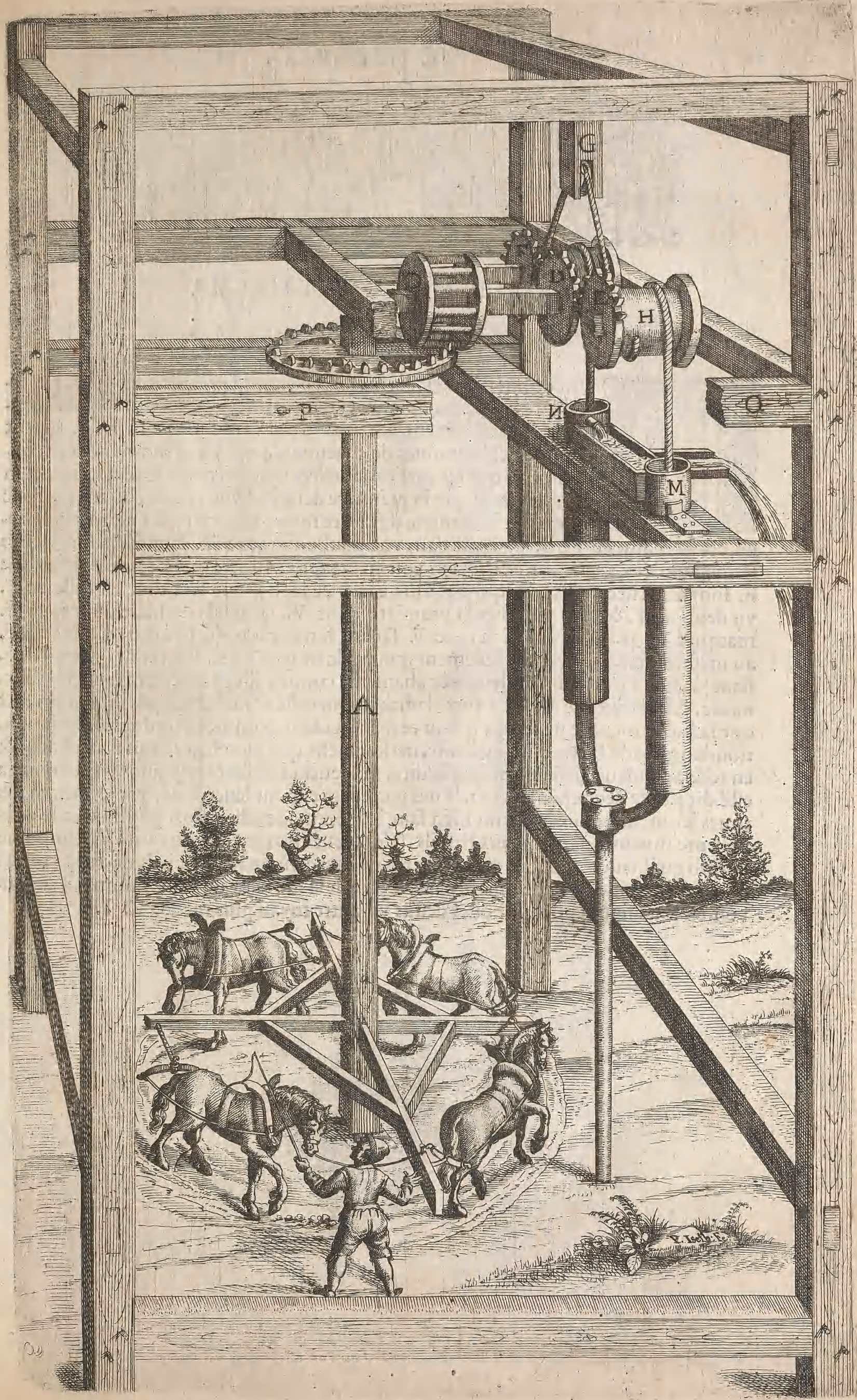




\section{Livre premier,}

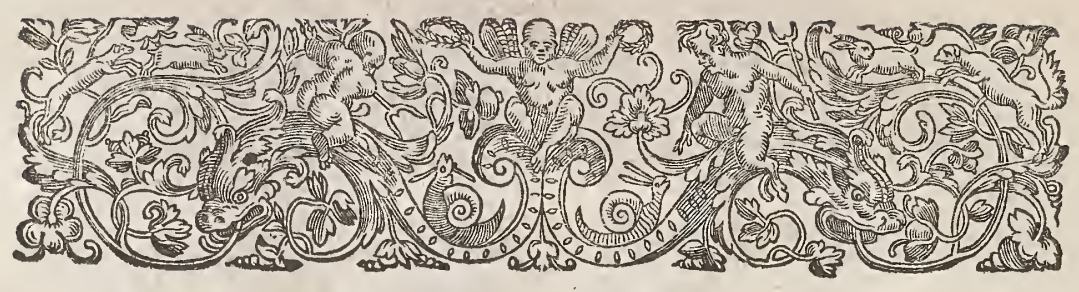

PROBLESME QUATRIESME.

\section{Plan de lortograpbie de la precedente machine.}

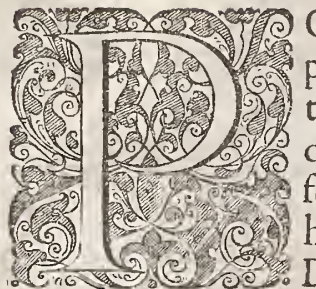

Our donner plus facille intelligence de la precedente figüre, i’ay reprefenté icy le plan delortographie,à fin que par iceluy lon puiffeentendrele mouuement \& rencontre des trois rouës E. D. F. foyent donques lefdites roues de chacune 9 .dents en la moitiédela circonference, \& que les parties dentelees d'icelles roues foyent tournees en haut, en forte que la premiere dent de l'vne, s'acroche auec la roue D. quand la derniere de lautre roue paffe outre, \& faut qu'ily ayevne corde marquee R. S. paffante dans vne pouliemarquee T. laquelle fera attachee ferme auxdeux axes,commeil fe peut voir en la figure,en forte que fi les dēts dela roue E. font acrochees, la corde qui eft ferme à laxe de ladite roue fera to urner celle de $F$. vn demi tour, \& fera prefenter la premiere dent $V$. quand la derniere de la roue $\mathrm{E}$. marquee X. paffera, \& ainfi la roue $F$. faifant fon demi tour, fera deftourner celle $E_{\text {r }}$ au mefme eftat que deuant, tellement que par le moyen de ce demi tour (alant \& venant) le feau de la pompe fe leuera \& abaiffera, comme fi ceftoit vne maneuelle tournante, \&la difference qu'il y a entre ladite maneuelle \& cefte prefente inuention, eft que ladite maneuelle ne leuent le feau perpendiculaire,comme fait cefte prefente inuention, laquelle eft beaucoup meilleure, mefmement que pour leuer l'eau fi treshaut, \& en telle abondance, il faudroit que lefdites maneuelles fuffent trespuiffantes, comme a efté dit par cy deuant,\& aux machines precedentes pour leuer leau, par le moyen des roues à eau, fil lon ne pourroit bien faire lefdites maneuelles, lon pourra vfer de cefte prefente inuention, quand aux bareils, fi lon veut efpargner la quantité de cuiure ou plomb qu'il faudroit auoir, en les faifants de treze où quatorze pieds de long, on les pourra faire feulement de quatre pieds long, dela groffeur du feau, comme il fe peut voir en cefte figure, puis emboiter deffus vn autre tuyau plus menu. Z. Y. 


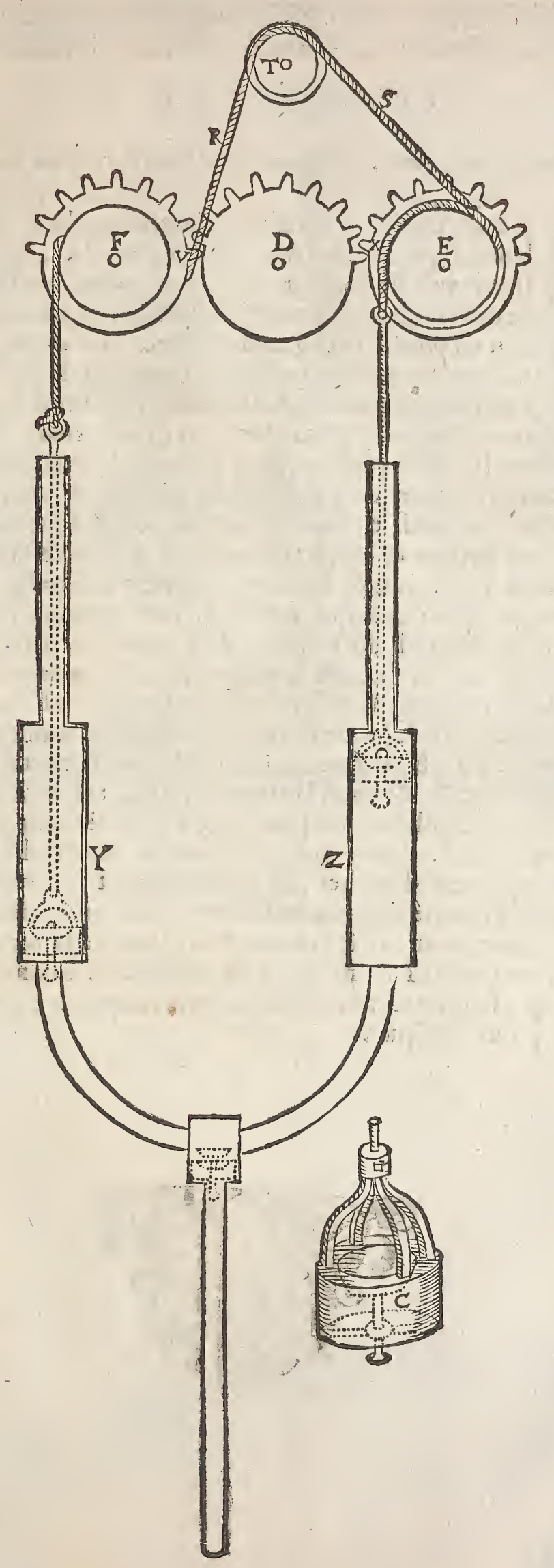




\section{Liure premier.}

\%

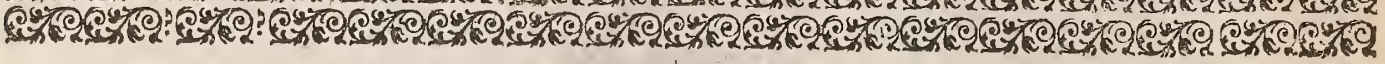

PROBLES M E V.

\section{Pour faire esleuer partic de l'eau d'vne fource, cing ou fix pieds haut.}

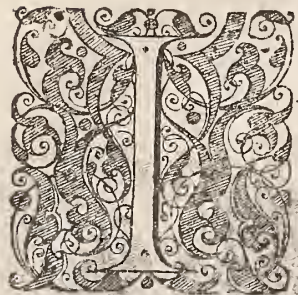

I Y A PLUSIEURS maifons \& iardins, dont les fituations font plus hautes que les fources voifines, \& sil y a quelque pente aufdites fources de fix ou fept pieds, l'on pourra hauffer partie de ladite eau, ce qui eft vneinuention fort fubtille, laquelle eftant bien entendue, l'on en pourra tirer grande utillité. Soit donques la fource conduite à yn lieu ou il y aye fix ou fept pieds de pente, puis foit fait deux A. \& B. lefquels feront trois ou quarre pieds en quat fermez de tous coltez marquez hauteur, \& celuy de bas huir pouces \& en quarre celuy A. aura demi pied en 作 cinq pieds, \& y aura vn receptacle au deffus du vaiffeau ' $A$. 'màrqué $C$. auquel entre. ra l'eau de la fource. Soit auffi les deux tuyaux K. \& E faits en forte que par celuy E. leau de la fource entrera \& émplira le vaiffeau A. puis eftant plain, on le bouchera auec le bouchon F. \& l'eau de la fource montera iufques au bout du tuauy K. lequel bout fera vn peu plus haut que celuy E. puis entrera dans le vaiffeau B. pariceluy tuyau K. \& au deffus dudit vaiffeau B. il y aura vn tuyau M: foudé lequel paffera par deffus le vaiffeau. A. \& auffi le recepracle, \& fera recourbé en bas, \& foudé contre le haut dudit vaiffeau $A$. tellement que lair du vaiffeau B. puiffe entrer par ledit tryau au vaiffeau $A$, \& puiffe contraindre l'eau de monter par le tuyau N. comme a efté demonfté au Theorefme 6. \& ainfi quand le vaiffeau $A$. Fera vuide, il faudra rouurir le tuyau E. \& alors l'eau de la fource r'entrera dedans iceluy vaiffeau, \& faudra auffi ouurir le robinet $R$. pour laiffer efcouler leau du vaiffeau $B$. dehors, $\&$ alors que le vaiffeau A. fera remply, \& celuy B. vuide, il faudra faire comme par deuant, \& l'eau montcra derechef, \& ainf ouurant \& ferrant les deux vaiffeaux comme a efté dit, la moitié de l'eau de la fource, montera cinq pieds plus haut que ron origine, \& l'autre moitié defcendra en bas, quand à̀ la recourbeure du tuyau. K. celaeft fait pour euiter que l'air ne forte du vaiffeau B. quand l'eau entrera dedans, faut auffi noter que la monteure de charpenterie n'a point efté faite à ce prefent def́reing pour ne le point ofufquer.

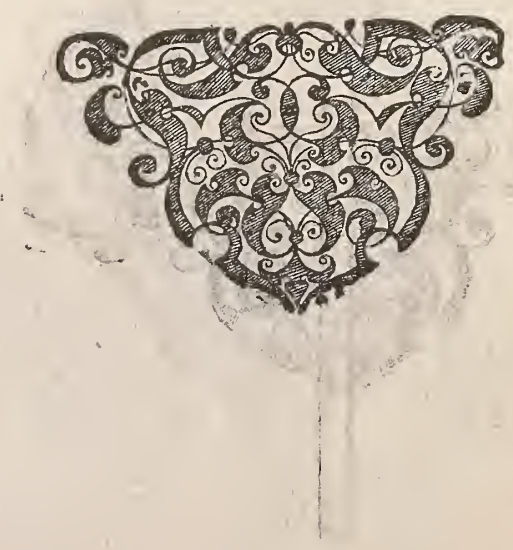




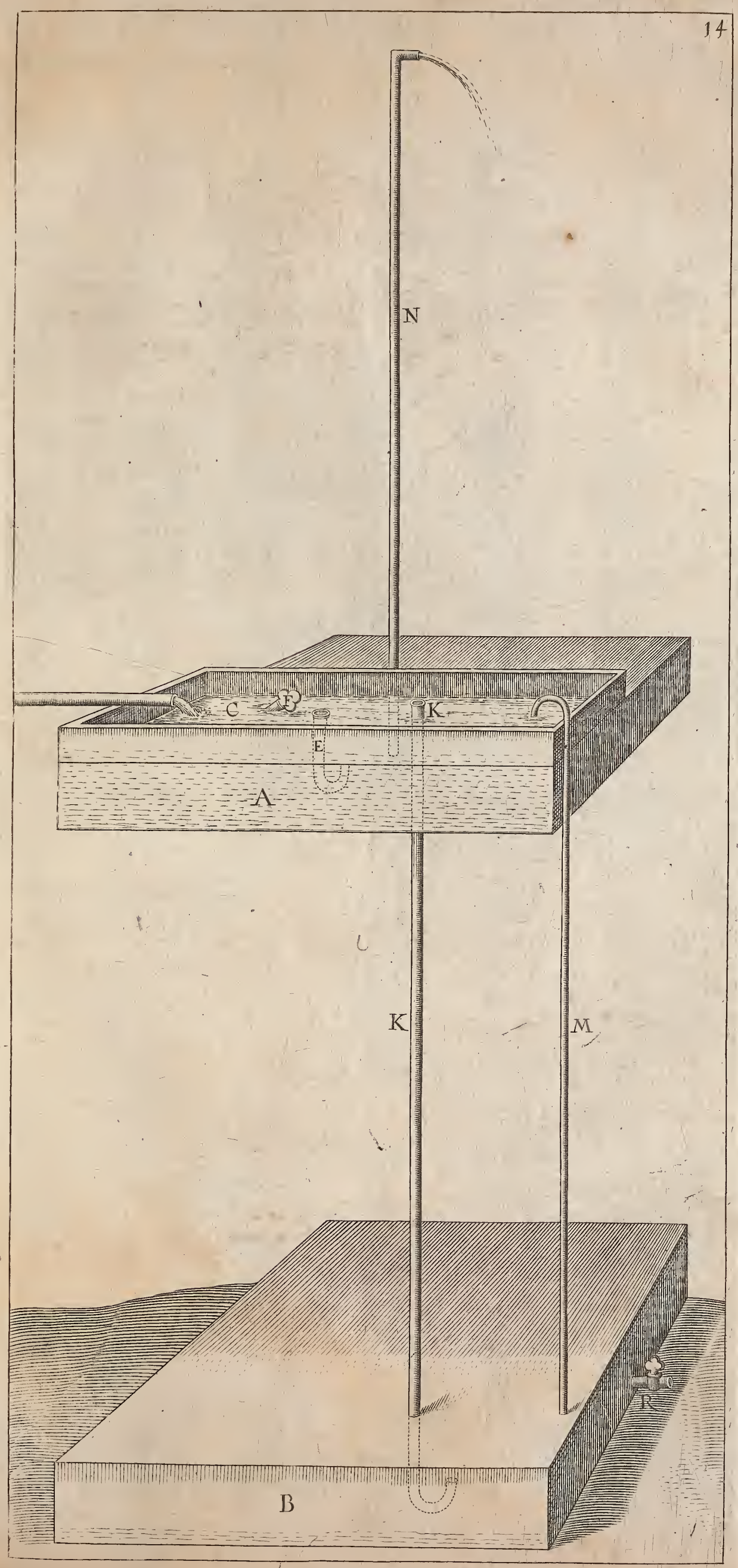




\section{Liure premier.}

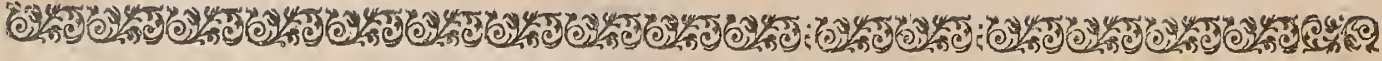

\section{P R O B L E S M E V I.}

\section{Machine fort fubtille, par laquelle les vaiffeaux de la precedente souurent \& ferrent d'eux mejmes, par le moyen de leau.}

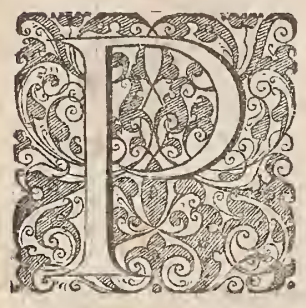

A R̂ la precedente il a efté monftré de leuer partie de l'eau d'vne fource, \& d'autant que c'eft vnegrande fubiection d'ouurir \& ferrercontinuellement les vaiffeaux, iay trouté vne inuention, laquelle par laide feulement de la mefme eau, lefdits vaiffeaux fe pourront ouvrir \& ferrer toufiours à propos, laquelle fe fera en cefte forte. Soit. premierement les vaiffeaux A.\& B.comme en la precedente, \& mefmement tous les tuyaux, \& au bour de haut de ceux E.\& K.ily aura deux robinets faits \& pofez en forte auec deux branches ou vergettes de fer, ou cuiures marquees C. D. \& deux regiftres ioints à icelles marquees F. G. aufquels regiftres la corde $M$. N. fera atachee, \& quand ladite corde fe tirera du cofté $\mathrm{N}$. alors il faut que le robinet $\mathrm{E}$. fe ferme, \& que celuy D. s'ouure, \& au contraire, quand l'on tirera lacorde du cofté M. le robinet E. s'ouurira, \& K. fe ferrera, 11 y aura auffi vn robinet au bas du vaiffeau $B$. lequel fera iuftement foubs $l_{a}$ verge $O$. P. lequel s'ouurira auffi auec celuy E. par le moyen du regiftre Q. apres fauc que la corde $M$. N. paffe par la poulie R. \& qu'elle foit attachee à vn perit vaiffeau de cuiure S. lequel fera fait de forte que quand il fera plain, il renuerfera fon eau, \& eftant vuide il fe rehaufera comme la figure le monftre, \& pour faire que ledir vaiffeau face c'eft effect, il faudra qu'il foit pendu entre deux piuots, vn peu plus bas que le millieu, \& que le fond foit de pefanteur pour tenir ledit vaiffeau comnic la figure monttre, \& quand il fera plein d'eau alors le haut eftant plus pefant que le bas, il renuerfera fon eau \& faudra à lautre colté de la machine auoir vne autre poulie marquee $T$. à laquelle fera paffee la corde attachee au contrepois $V$. lequel ferabalancé auecle vaiffeau $S$. en forte que ledir vaiffeau eftant vuide, alors le contrepois le fera haufer, \& par confequent ouurir le robinet E, \& auffi celuy qui eft au bas du vaiffeau $B$. \& quand ledit varffeau $S_{+}$fera à moitié plain, alors il attirera ledit contrepois, \& fera retourner les robinets comme a efté dit, \& ainfi le tout eftant bien aiufté, fi l'eau de la fource tombe au petitrẹceptacle, elle entrera dans le vaiffeau A. par le robinet E. \& quand ledit vaiffeau fera plain, alors l'eau montera audit receptacle, iufques au tuyau X. \& de là tombera dans le petit vaiffeau S. lequel eftant demi plain, atrirera le contrepois V. \& fermera (comme a efté dit) les robinets $\mathrm{O}$ \& \& E. \& ou urira celuy $\mathrm{K}$, alors l'eau entrant dans le vaiffeau de bas fera monter celle de $\mathrm{A}$. au vaiffeau $\mathrm{Z}$. comme a efté monftré par la precedente, \& apres que le vaiffeau B. fera plain, \& celuy A. vuide, alors leau montera derechef au receptacle, iufques au tuyau X. \& tombera dans le vaiffeau S. jufques à ce qưil renuerfe, alors le contrepois $V$. retirera ledit vaiffeau en haut, \& remettra les robinets en leur premier eftat, \& continuera ce mouuement fans autre aide que ladite eau. Et fi lon defire auoir de l'eau plus haut que cinq ou fix pieds, alors il faudra faire encores vne machine foubs le tuyau $L$. du vaiffeau de haut, \& alors la moitié de celle qui entre audit vaiffeau Z. montera encores plus haut, \& fi c'eft que leau foit abondante à la fource, on la pourra faire monter autant que bon femblera par cefte inuention. 


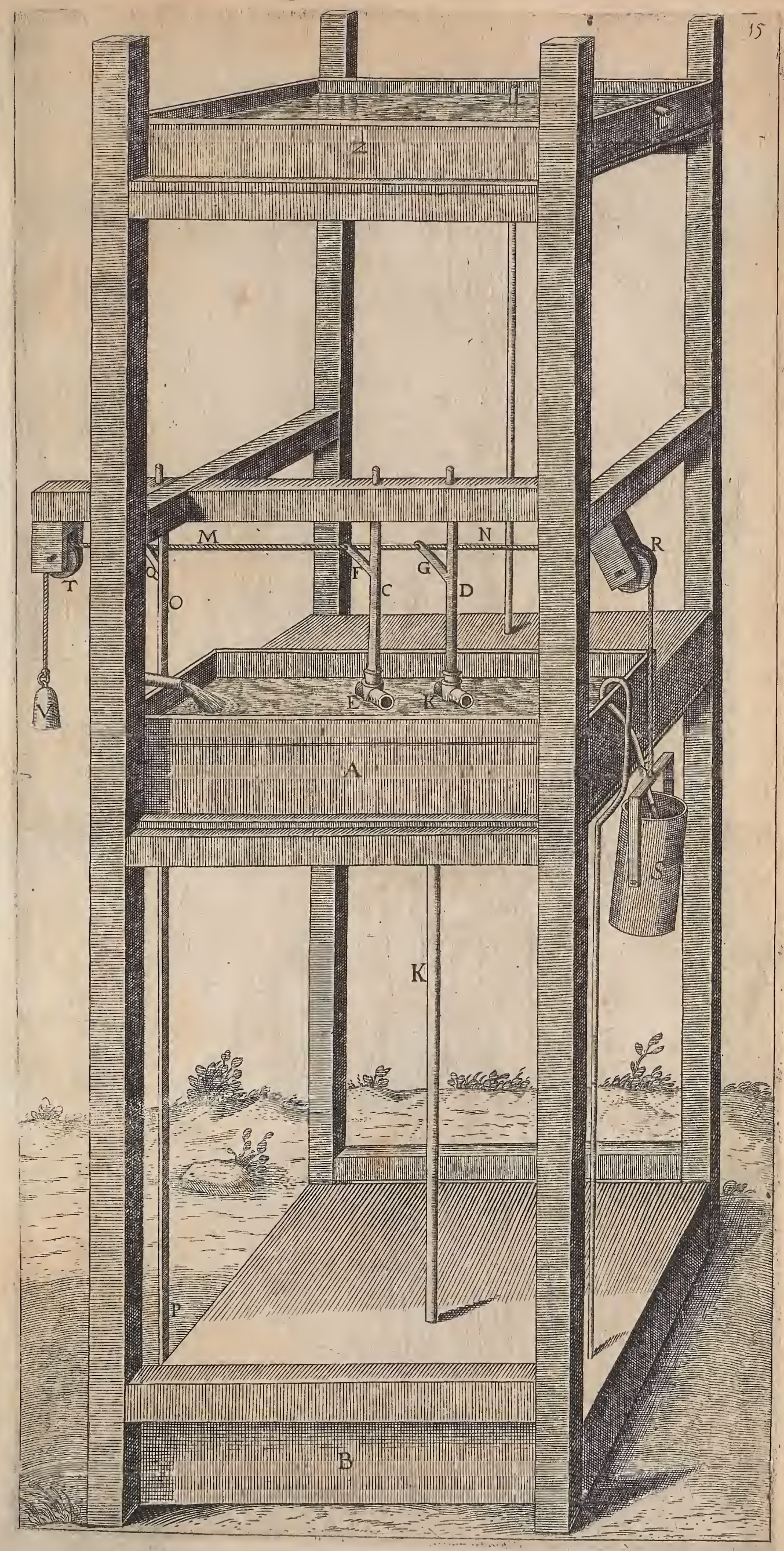


$-=1$ mTा
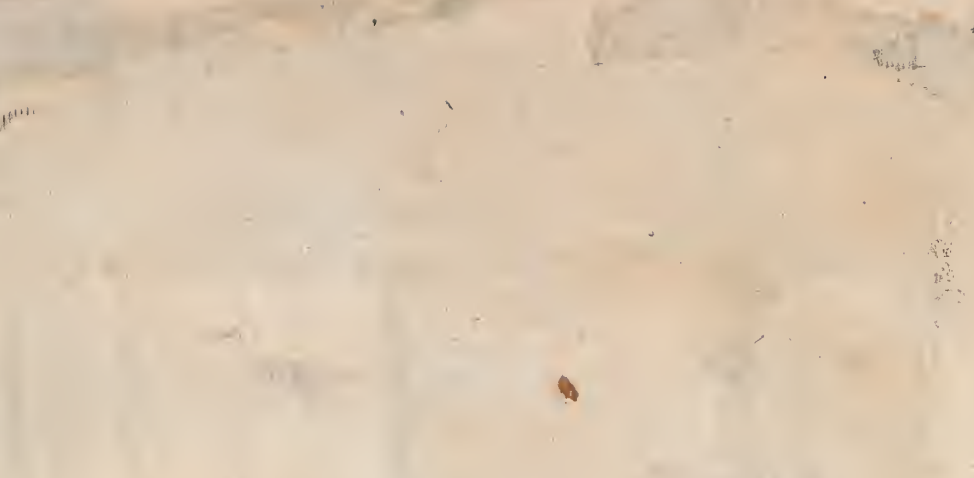

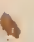

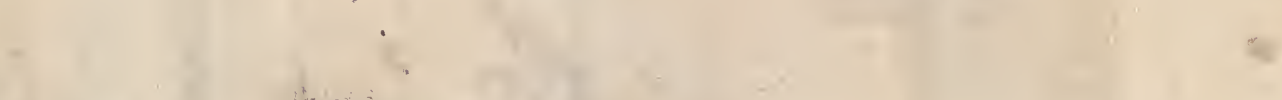

$\therefore \quad$ 年

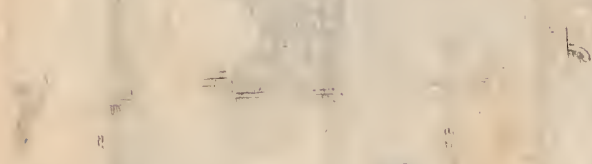

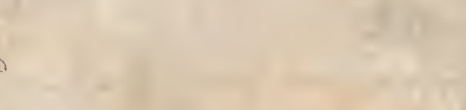

tent

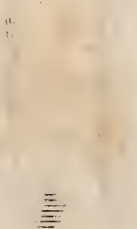

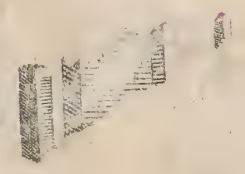

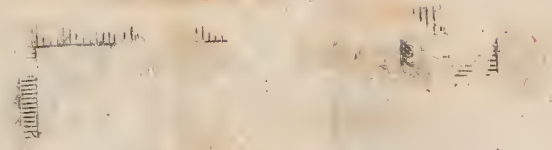

.

!

$\cdots$

-

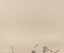

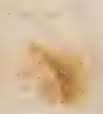

$\equiv$
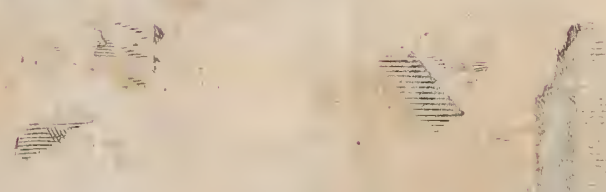

$=$<smiles>C1#CCCCCC1</smiles>
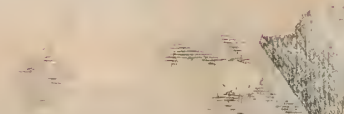

7

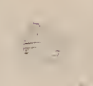

$+4$

(4)

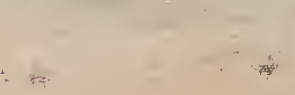




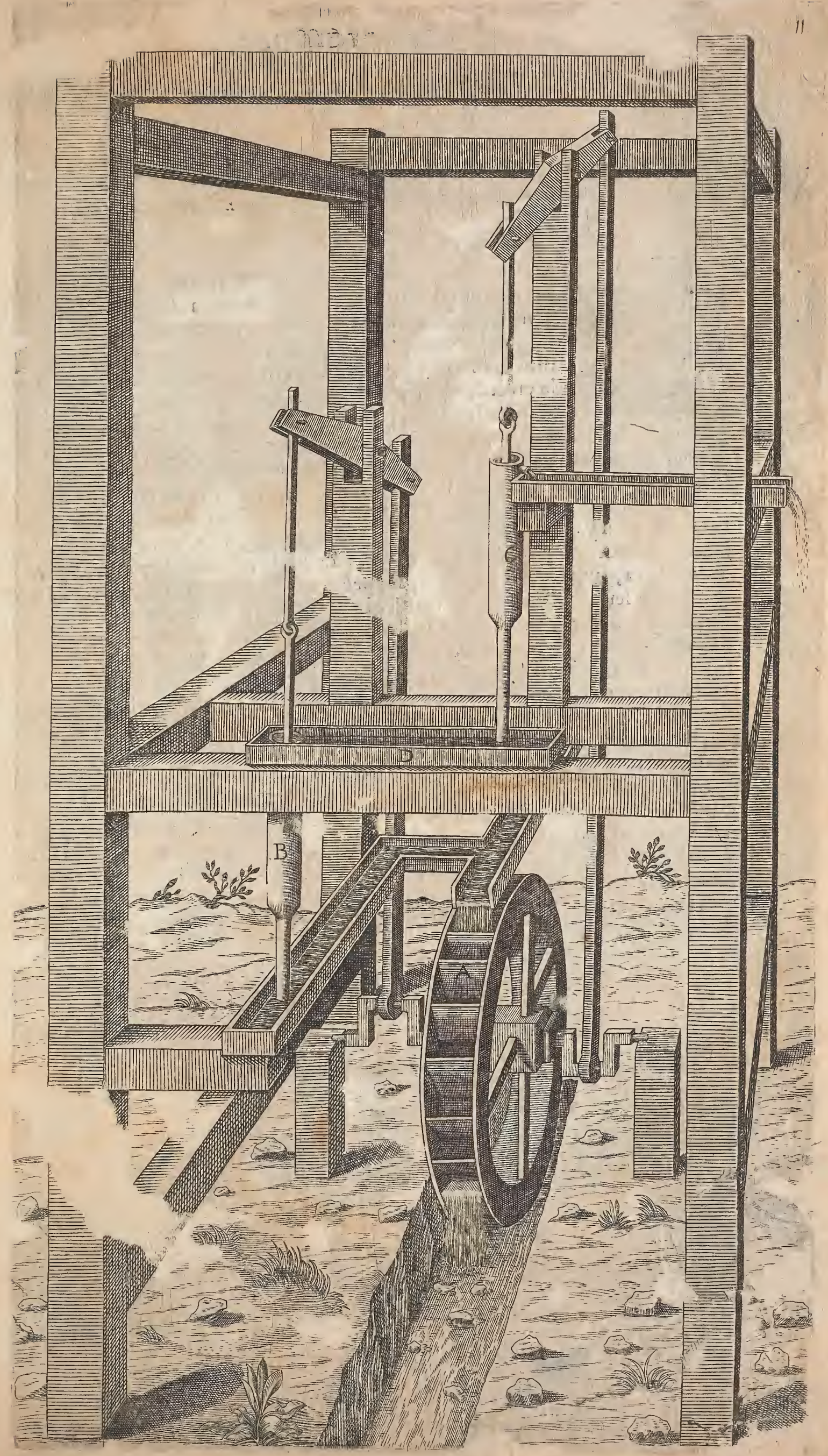




\section{Liure premier.

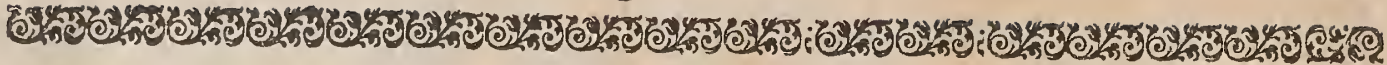 P R O B L E S M E VII.}

Pour faire vne Orologe ausec le cours divne fontaine naturelle laquelle pourra faire fon cours trefiuste, fans estre fubiette à estre montee cournellement.

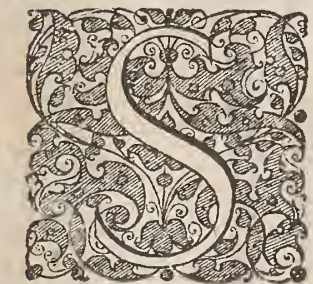

Orr le cours de la fontaine au tuyau marqué $A$, lequel fera gros par dedans, viron comme vne plume à efcrire, dont l'eau tombera dans le vaiffeau B. auquel fera deux tuyaux, fçavoir vn marqué C. foudé contre le fond dudit vaiffeau, auquel il y aura vn petit robinet D. apres il y aura vn petit vaiffeau tresbuchant, (comme en la precedente machine) marquee $E_{\text {. \& ledit vaiffeau fera attachéa }}$ vn petit levier marqué F. G. fait comme la figure demonftre, fçavoir auec vne charniere pour ployer feulement d'vn cofté, \& le bout dudit leuier fera acroché dans vne des dents d'vne grande roue, marquee H. I. en forte que le vaiffeau E. baiffant, le bout G. leuera la dent acrochee, vn peu plus que d'vne dent, à fin que le plus grand leuier L. tombe dans la prochaine dent, \& face arrefter ladite roüe $H$. I. il y aura auffi vn contrepois marqué $O$. attaché au leuier $F$. G. pourabaiffer le bout G. quand le vaiffeau $E$. fera vuide, \& ainfi l'eau tombante dans ledit vaiffeau, quand il fera vn peu plus pefant que le contrepois $O$, alors il s'abaiffera , \& fera leuer le, bout G. \& hauffer ladite roüe H. d'vne dent, \& l'eau to mbante toufiours emplira ledit vaiffeau, \& le fera renuerfer, \& alors le contrepois O. lequel fera plus pefant que le vaiffeau vuide, rabaiffera le bout du leuier G. \& celuy d'enhaut L. tiendra la roüe en eftat quelle ne pourra retourner, \& faudra que ladite rovë aye foixante dents, \& auffi que l'eau qui tombe dans le vaiffeau E. foit tellement aiufté auec le robinet $D$. que chafcune minute d'heure, ledit vaiffeau fe paiffe renver, fer, \& par ce moyen la roüe H. I. fera vn tour en vne heure, \& apres l'on pourra faire qu'il y aura vn pignon à l'arbre de ladite rouë ayant fix dents, lequel mouuera vne roúe de feptantedeux dents, \& par ce moyen la monftre de haut $M$. monftrera le cours de douze heures, \& celle de bas d'vne. Et quand ledit Orologe fera bien aiufté, elle continuera long temps fans varier, faut auffi noter qu'il faut que l'eau du vaiffeau, B. foit de la hauteur du tuyau P. à celle fin, que ladite eau tombe toufiours efguallement dans le vaiffeau tresbuchant, \& pour ce faire, faudra quil en tombe vn peu plus dans ledit vaiffeau qu'iln'en forte par le tuyau C. \& le furplus fortira par le tuyau

PR O.

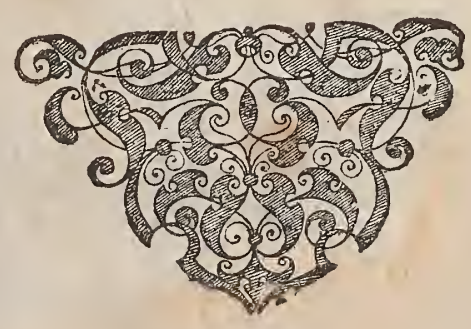




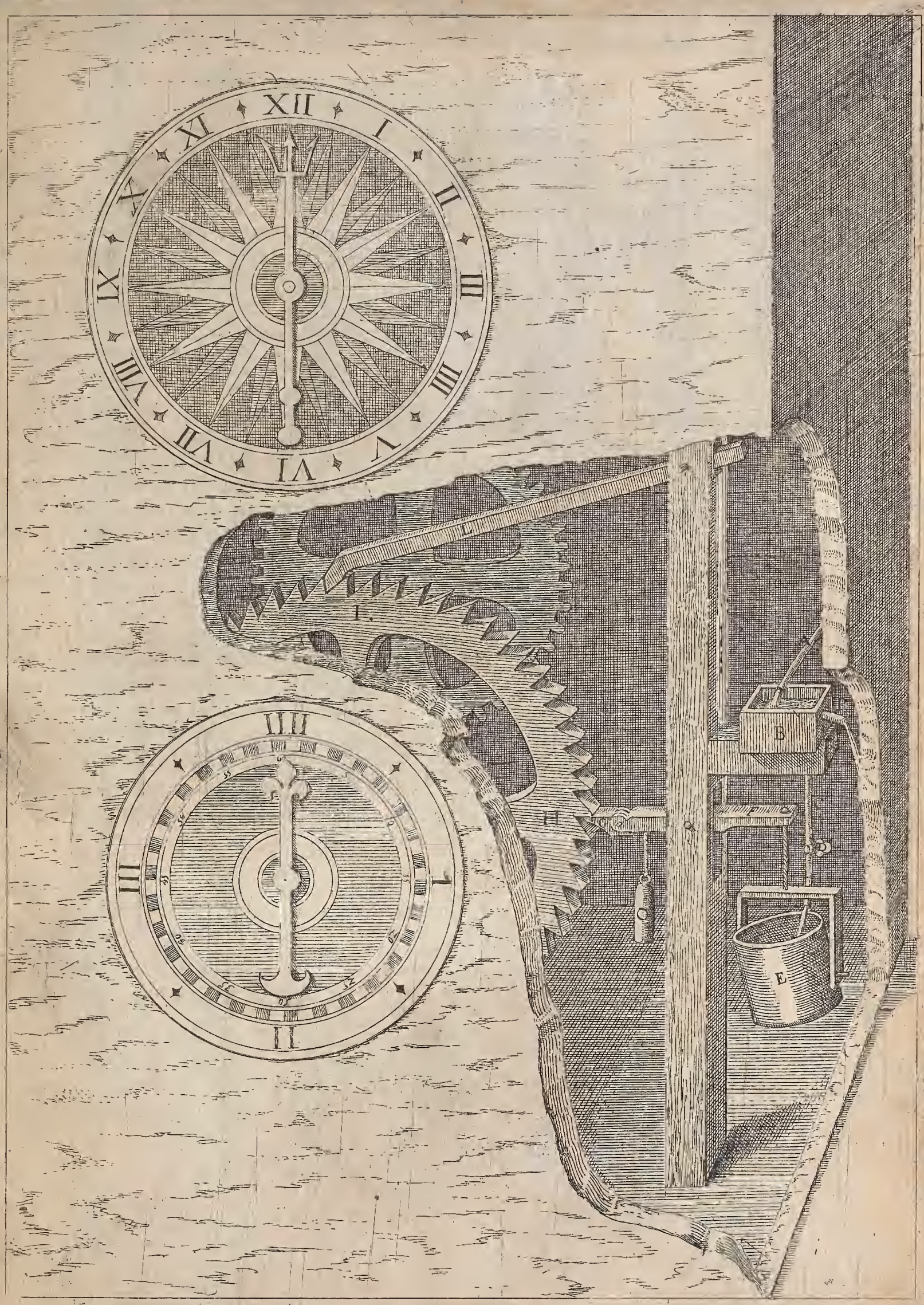




\section{Liure premier.}

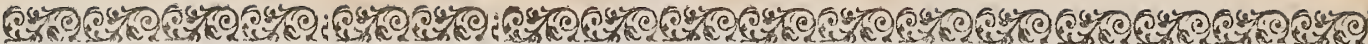

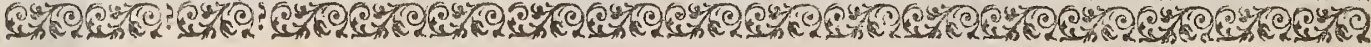

\section{P R O B L E S M E VII.I.}

Autre maniere d'Orologe d'eau.

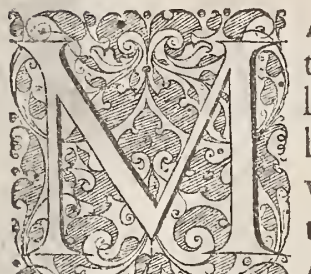

A s s'il n'y auoit point de fource vifve, \& quelon vouluft mefurer le temps auec l'eau, lon fera vn vaifeau de cuiure ou plomb, comme la figure A. lequel tiendra viron vn muy d'eau, ledit vaiffeau fera bien quarré, \& vn peu plus haut que large, dans lequel fera vn petic vaiffeau de cuiure marqué $F$. auffi quarré bien clos, \& foudé de rous les coftez. Lequel fervira pour flotter deffus l'eau du vaiffeau A. apres faut auoir vn fifon fait commela figure B. C. D. lemonftre, lequel paffera à trauers vn cuyau de cuiure, qui fera au milieu du peric vaiffeau, \& faut que le dit ruyau paffe de part \& dautre dudit vaiffeau, \& le fi fon entrera dedans avec vn peu de forcef, \& faut auffi que le bout dudit fifon puiffe trēper dedans l'eau du vaiffeau. A. \& en haut au point C. il y aura vne corde atachee; paffante par deffus la poulie L. \& à laucre bout fera ataché le contrepois E. \& au bout de laxe, du cofté I. fera atachee vne efguille laquelle monftrera les heures en la monftre O. P. \& apres que lon aura rempli le vailfeau A. Ion pofera le vaiffeau F. defís, comme a efté dit, \& auffi le fifon \& contrepois, puis lon atrirera l'eau dudic fifon par le bout D. auec la bouche, \& d'autant que ledit bout eft plus bas que le niueau de leau du vaiffeau A. la. dice eau aura fon cours, \& tombera dans vn aurre vaiffeau. $H$. \& à mefure que l'eau dudit vaiffeau s'abaiffe, le petit vaiffeau. F. s'abaiffera auec le fifon, ce qui fera cau fo de farre courner la poulie, \& par confequent lefguille de la monftre, \& pour aiufter le cours des heures, faudra al nger ou acourfir le fifon dans le tuyau de euiure du petit vaiffeau, car en pouffant ledic fifon vn peu d'auantage dans l'eau, elle courra plus vifte, \& au contraire le retirant, elle fe retardera, faut auifi noter, que pour aller tort iufte fera de befoing d'aiufter vi pecit cuyau au bout $D$. dont l'extremiré du bout ou eft le perit percuis par ou fort leau, fera d'or fin, à celle fin que ledit trou ne fe bou. che de rouille, ce qu'il fe feroit, s'il eftoir de plomb ou cuiure, \& quand l'eau du vaiffeau A. Cera prefque vuide, on la fera remonftrer auec vne petite pompe marquee $E$.

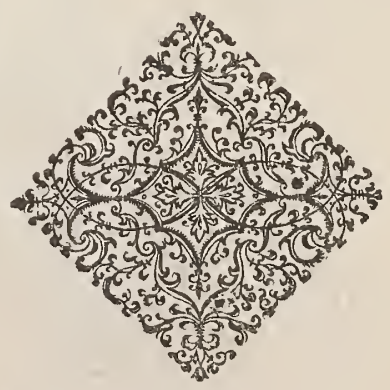




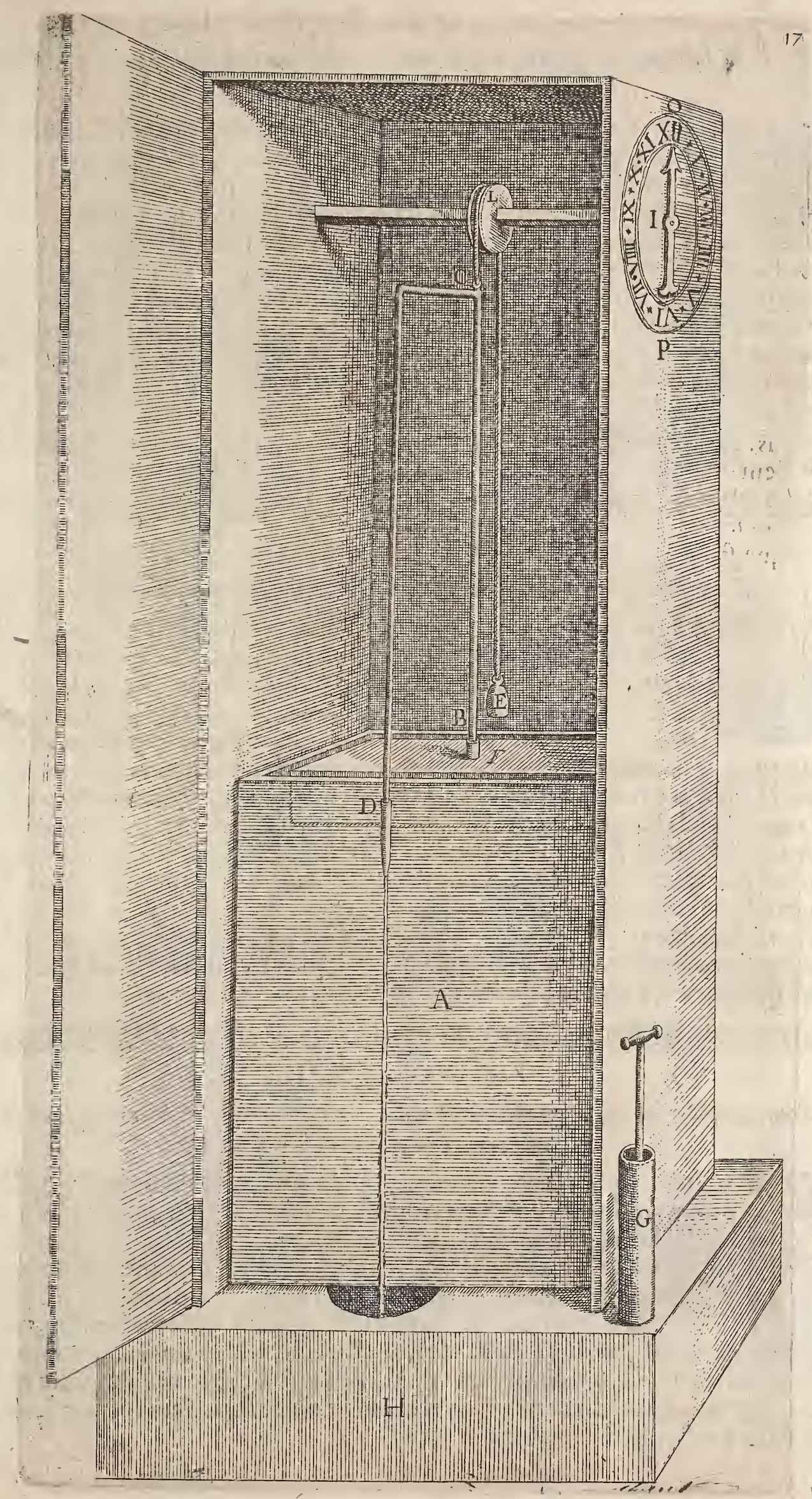




\title{
Livre premier,
}

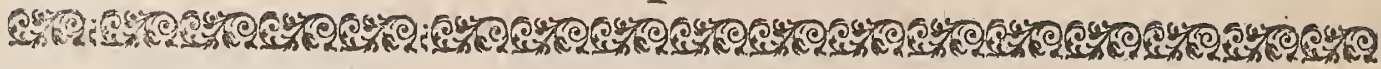

P R O B L E S M E IX.

Pour faire vn vaifleau, auquel mettant de l'eau par force, fortira puis apres auec grande violence.

\begin{abstract}
Or vn vaiffeau decuiurebien rond \& de force affez capable pour foultear( (2) nir l'esfort de l'air, \& qu'il foit bien clos \& foudé de tous coftez, apres y" 6. D. . faudra fouder deux tuyaux, f̧̧auoir A. B. \& C. D. en forte que cha-

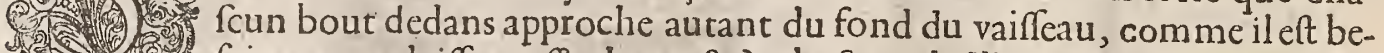
foing, pour laiffer paffer l'eau, \& à chafcun defdits tuy aux, il y aura vne clef ou robinet pour ferrer l'eau, quand elle fera dedans, laquelle on mettra auec une Seringue par le tuyau C. D. \& faudra bien aiufter le bout de ladite Seringue au bout C. à celle fin qu'en pouffant l'eau dedans elle ne reforte par la iointure, \& à l'inftant qu'on là pouffee dedans, il faudra ouurir lá clef $G$. puis la referrer auffi toft quili n'y a plus d'eau dedans la Seringue, \& ainfi quand l'on voudra faire fortir l'eau, on tournera la clef ou robinet $\mathrm{F}$. puis elle fortira par le tuyau $\mathrm{A}$. (duquel le trou fera fait, auffi menu que la groffeur d'vne efplingue, ) douze ou quinze pieds de haut, ce qui donnera plaifir à voir.
\end{abstract}

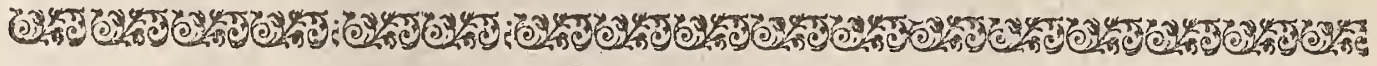

\section{P R O B L E S M E X.}

Pour contrefaire la voix des petits Oifeaux par le moyen de l'eau, Es lair.

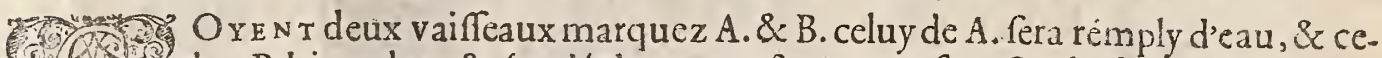
G luy B.bien clos, \& foudé de tous coltez, puis faut fouder le tuyau C. D. vn S bout contre le fond du vaiffeau A. \& l'autre paffant en la partie fuperieu. (1) E Tue le bout D. foit autant diftant du fond de B. comme il eft Lefoing pour laiffer paffer l'eau, faudra auoir vn robinet marqué $F$. audie suyau pour ouurir \& ferrer quand befoing fera, faudra encores faire vn cuyau G. H. par lequel l'aër paffera à trauers ledit vaiffeau. A. ou bien fera conduit au lieu ou l'on voudra faire chanter les Oyfeaux, audit bout rera aiufté vn petit fifflet femblable à ceux que font les faifeurs d'Orgues, pour reprefenter le chant d'vn Rofignol, \& ledit fiffer trempera dans l'eau, comme il fe peut voir en la tigure particuliere $K$. \& ainfi quandl'on ouurira le robinet F. I'eau defendra au vaiffeau de bas, \& l'air qui eft au. dit vaiffeau fortira par le tuyau G. $H_{*}$ lequel fera fiffler le fifler qui eft au bout dudie tuyau, \& aupres d'iceluy, l'on pourra pofer vn arbriffeau artificiel, deffus lequel l'on met. ra quelques oyfeaux de bois où metal peints comme le naturel.

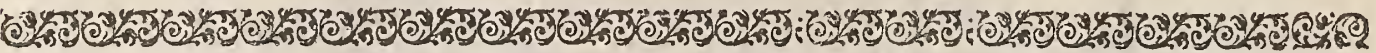 P R O B L E S M E X I.}

Pour aiouster auf fuldit mounement on Cigne, ou quelque autre $O y$ feau, lequel boirs autant d'eau comme on luy donners.

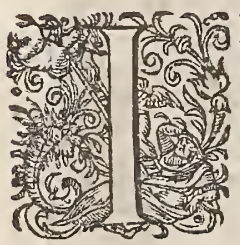

L fe pourra encores fairequau vaiffeau A. il y aura vn Cigne fait de metal comme il eft defigné en la figure $\mathrm{M}$. lequel boira autant d'eau commeon luy prefentera foubs le bec, \& pour ce faire, il faudra bien clorrele vaiffeau $A$. de tous coftez, \& faire vn tuyau marqué $N$. au bout de haut duquel ily aura vn petit receptacle, \& l'autre bout aprochera autant du fond dudit vaiffeau, comme il faut pour laiffer paffer leau, \& au Cigne il y aura vn tuyau marqué $O$. foudé deffus la fuperficie dudit vaiffeau, en forte quequand l'eau defcendra parle Robinet F.au vaiffeau de bas, laër entrera au vaiffeau A. par le tuyau, qui refpondra aubec du Cigne, \& fi lon met de l'eau foubs le bec dudit Cigne, il l'attirera en la plaçe de leau qui defcend. 


\section{Liure premier.}

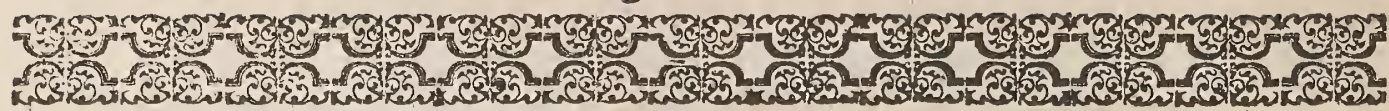

PR O B L E S E XII.

Pour faire une Macbine, laquelle aura moumement de Joy-mefme.

Les quatre elersents dot la machine est compofec font enteridu es, faroor la matiere aôt elle est com. pofeepour la ierre có leas de dedans posr leau o Pair anfsi fast on of felt dedans le vaifears. comme anfis fart le fors, farfani mon ter es defiédre la ballio.

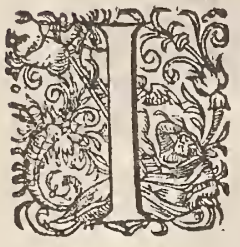
L y a eu plufieurs hommes lefquels fe font trauaillez à la recherched'yn mouuement qu'ils ont appellé ( fans le congnoiftre) per̂petuel, ou fans fin, chofe affez mal confideree \& mal entendue, dautant que tout ce qui a commencement, eft fubiect à auoir vne fin, \& faut applicquer ce mor de perpetuel ou fans fin à Dieu feul,lequel comme il na eu commencement, ne pourra auffi auoir fin, tellement que cefte follie \& orgucil aux hommes, de fe vouloir faire acroire de faire des ouures perpetuelles, veu que eux mefmes font mortels, \& fubiets à vne fin, ainfi feront toutes leurs ouures, tellement que ie laifferay ces mots de perpetuel ou fans fin, \& monftreray icy la fabrique d'vne machine qui s'agift de foy-merme, pourueu qu'elle foit encretenue des quatres eflements dont elle eft compofee. I'ay demonftré au Theorefme cinquiefme,comme l'eau monte par l'aride du fou, plus haut que fon niueau. I'ay auffi demonftré à la premicre definition, comme toue chaleur natarelle peut eftre dite feu eflementaire,ainfi cefte difpofition nacurette de la chaleur, \& du defaut de chaleur, fervira de contrepois, pour faire monter leau, ie dis difproportion pour agir, dautant qu'il faut en tout mouuement, que le fort emporte le foible, autrement les chofes eftant en efquilibre ou en proportion efgualle, il ny pourra auoir mouuement, doncques cefte difproportion eftant en lair efchauffé par la chaleur du Soleil, fera la caufe du mouuement, comme il fe pourra voir icy par la fabrique. Soit vn vaiffeau de plomb ou de cuiure de viron vn pied \& demi en quarré bien clos \& foudé de tous les coftez auquel il y aura vn tuyau au milieu marqué $D$. E. le bout $E$. approchera du fond du vaiffeau, comme il eft befoing pour laiffer paffer leau, \& lautre bout $D$. Tera bien foudé contre le haut du vaiffeau, \& y aura auffi vn foufpiral marqué $F$. apres faut efleuer les deux coftez de la machine $N$. M. en forte que laxe $O$. auec la poullie G. puiffe tourner facillement, \& monftrer au dehors du cofté $M$. le moutement de ladite machine, auec lefguille appofee contre laxe fufdite, faut auffi atoir la bordeure P. Q. foudee au deffus du vaiffeau laquelle feruira quand l'on voudra mettre deleau dedans le vaiffeau, \& auffi quand leau monte par le cuyau E. D. qu'elle ne s'efpan. de dehors, tout cecy eftant bien \& iuftement conftruir, lon verfera de leau dedans ledit vaiffeau, par le tuyau D. E. iufques a enuiron le tiers dudit vaiffeau, \& ourrira on le foufpiral $F$. quand on mettra ladite eau au vaiffeau, puis on le rebouchera tresbien, apres on aura vne pecite balle de cuiure fort legere marquee $\mathrm{L}$. laquelle puife flotter deffus leau, \& fera attachee par vn fillet, en forte que ladite balle puiffe hauffer \& baiffer dans le tuyau D. E. quand leau hauffe ou abbaiffe, faudra auffi que ledit fillet ou eft attaché la balle, paffe par la poulie G. au bout, duquel filet féra attaché vn petit contrepois $R$. comme le tout fe peut bien voir en la figure, apres faut pofer ladite machine dedans vne chambre, ou le Soleil du Mydi puiffe entrer, alors quand il fera vn peu de challeur, la balle fe hauffera, \& le contrepois s'abaiffera, qui fera caufe que lefguille tournera, \& monftrera la hauteur que la balle eft montee, \&e comme le temps fe refroidira, la balle s'abaiffera, \& ainfi comme le temps fe changera, ainfi la balle fe hauffera \& abaiffera, faut noter, que fi le vaiffeau eft vn pied \& demi de haur, \& eftant empli au tiers deau, reftera vn pied que la balle pourra hauffer \& baiffer, \& faifant la poulie de quatre pouces en diametre, alors elletournera vn tour, fi la balle fe hauffe iufques au bout $D$. tellement que diuifant la monftre en douze parties efgualles, chacune partie monftrera vn pouce, que la balle aura hauffé où abaiff́́. Quand à l'ýage de ladite machine, elle pourra feruir a remarquer les iours 
les plus froids ou les plâs chauds sorcar eftant ladite machine en quelque part de la chambre quede Soleif ne ldongle point deffus, alofs sta balle de cuiure fe hauffera felon la temperature du oür, carstistent fort chaud, la dite balle fe hauffera fort haut, \& fi au contraire il eft têmperé, sladite balle fe hauffera que fort peu, faut noter auffi, que quand lon metra leau dedans ladite machine. Il faut que ladice eau foit frefchement tiree d'vn puis oủ fontailine s '\&' incontinent qu'elle elt dedans, faut bien bou-

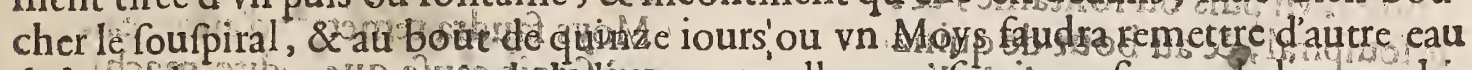

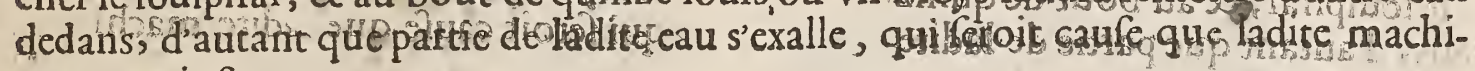
ne n'auroit fon mouuement.

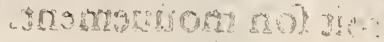

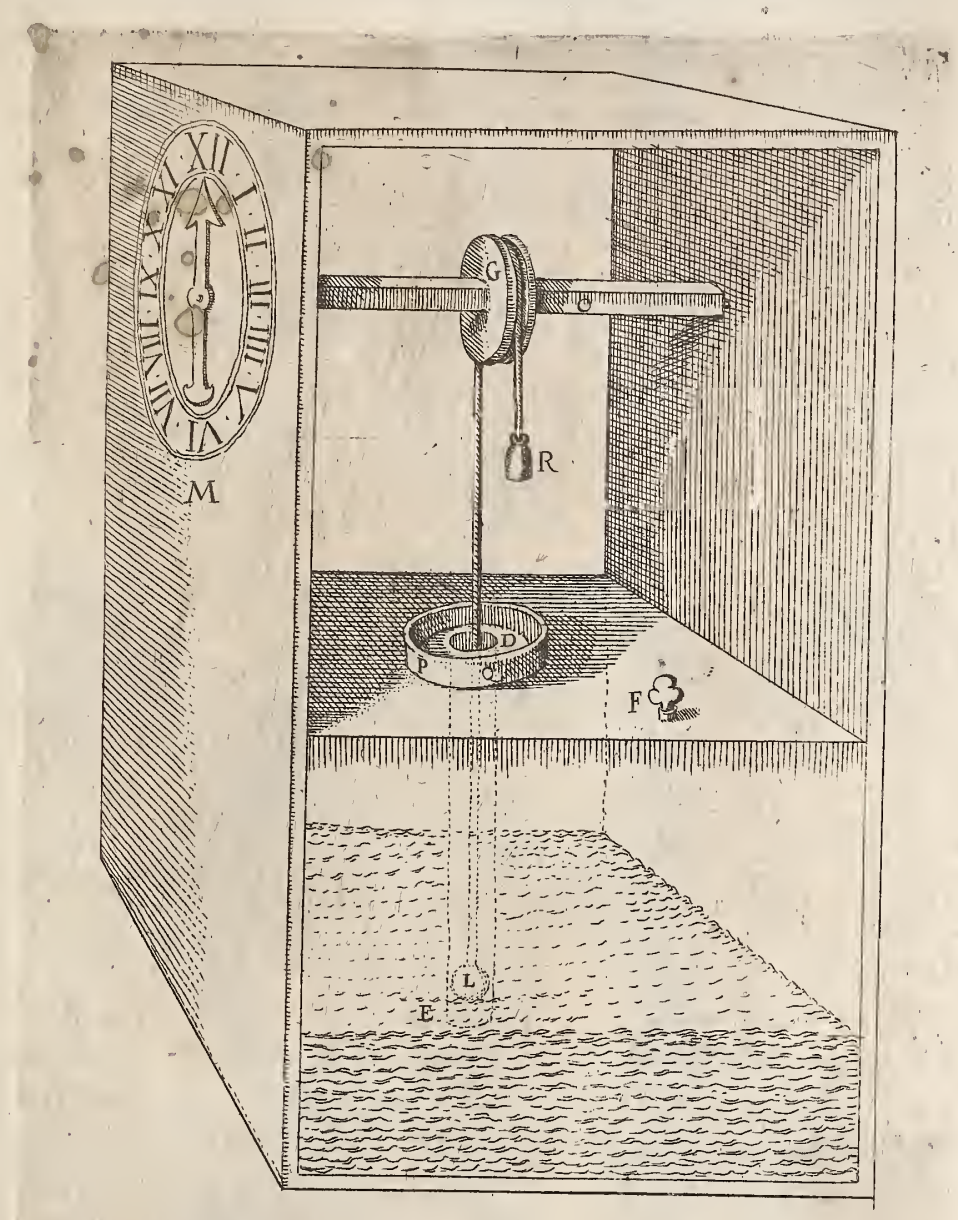




\section{Liure premier.}

14.

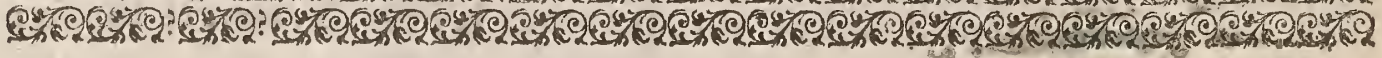

\section{P R O B L E S M E X I I I.}

\section{Macbine fort fubtile, par laquelle on pourra faire eslever vne eau. dormante.}

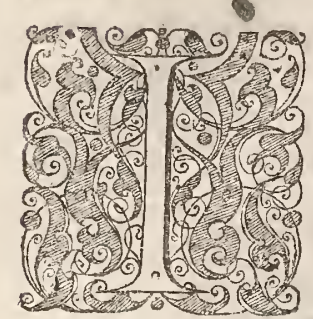

L a efté monfré parle precedent probleme, la fabriqué \& raifon d'vn mouuement continuel, de laquelle invention, iay prins la prefente machine, à laquelle lon pourrort attribuer le tilte de fontaine continuelle, à raifon que leau, laquelle de fa nature cherche le plus bas lieu, eft efleuee icy par le moyen du Soleil, cefte dise machine aura vn grand effect aux lieux chaûds, commel ETpaigne \& littalie, d'autant que le Soleil fe monftre en ces endroits, prefque tous les iours, auec grande chaleur, \& fpeciallement en Efté, la fabrique en fera telle, faut auoir quatre vaiffeaux de cuiure, bien foudees tout à l'entour lefquels feront chafcun viron vn pied en quarré, \& $\&$ huir ou neuf pouces de haut, lefdits vaiffeaux feront marquez A. B. C. D. \& y aura vn tuyau marqué E. poré fur lefdits vaiffeaux, auquel tuyau feront foudees quatre' branches marquees chacune branche par la lettre $F$. lefdires branches feront foudees au haur des vaiffeaux paffants iufques pres du fond de chafcun vaiffeau, faut apres au millieu du tuy au fouder vne foupape marquee G. faite \& pofee en forte, que quand leau fortira des vaiffeaux,elle puiffe ouurir, \& eftant fortie, qu'elle fe puiffe referrer, faut auffi auoir vn autre tuyau au deffoubs defdits vaiffeaux marqué $P$. auquel y aura aufi quatrebranches, lefquelles feront toutes foudees contre les fonds defdits vaiffeaux, \& auffi vne foupape marquee $H$. à laquelle il y aura vn tuyau au bout, qui defcendra au fonddeleau, laquelle fera dans vne Cifterne ou vaiffeau marqué $l$. il y aura auffi à l'vn des vaiffeaux vn trou ou efuent marqué M. ainfi faudra expofer la machine en vn lieu, ou le Soleil puiffe donner deffus, puis verfer de leau dans les vaiffeaux par le trou ou efvent M.laquelle eau fe communiquera à tous les vaiffeaux, par le moyen du tuyau P. \& faut que lefdits vaiffeaux ayent environ le tiers de leurs contenu deau, \& lair qui eftoit en la place de ladite eau, fortira par les foufpiraux 3. 4. 5. 6. apres faudra bien boucher tous lefdirs foufpiraux, en forte que l'air ne puife fortir defdits vaiffeaux, \& alors que le Soleil donnera fur ladite machine, il fe fera vne expreffion, à caufe de la chaleur, (comme a efté monftré au precedent problefme) ce qui caufera leau de monter de tous les vaiffeaux, au tuyau E. \& fortir par la foupape G. \& tuyau N. puis tombera dans le petit baffin $O$. \& de là dans la Cifterne $1 . \&$ comme il fera forti vne quantité d'eau par la violence de la chaleur du Soleil, alors la foupape G. fe referrera, \& apres que la chaleur du iour fera paffé, \& que la nuir viendra, les vaiffeaux pour euiter vacuiré, attireront l'eau de la Cifterne, par le tuyau \& foupape H. P. pour remplir les vaiffeaux comme ils eftoyent auparauant, tellement que ce mouuement continuera autant comme il y aura de l'eau à la Cifterne, \& que le Soleil donnera deffus les vaiffeaux, \& faut noter que les deux foupapes G. \& H. feront faites fort legercs , \& auffi qu'elles ferrent fort iuftes, fans que l'eau puiffe defcendre quand elle fera montee. 


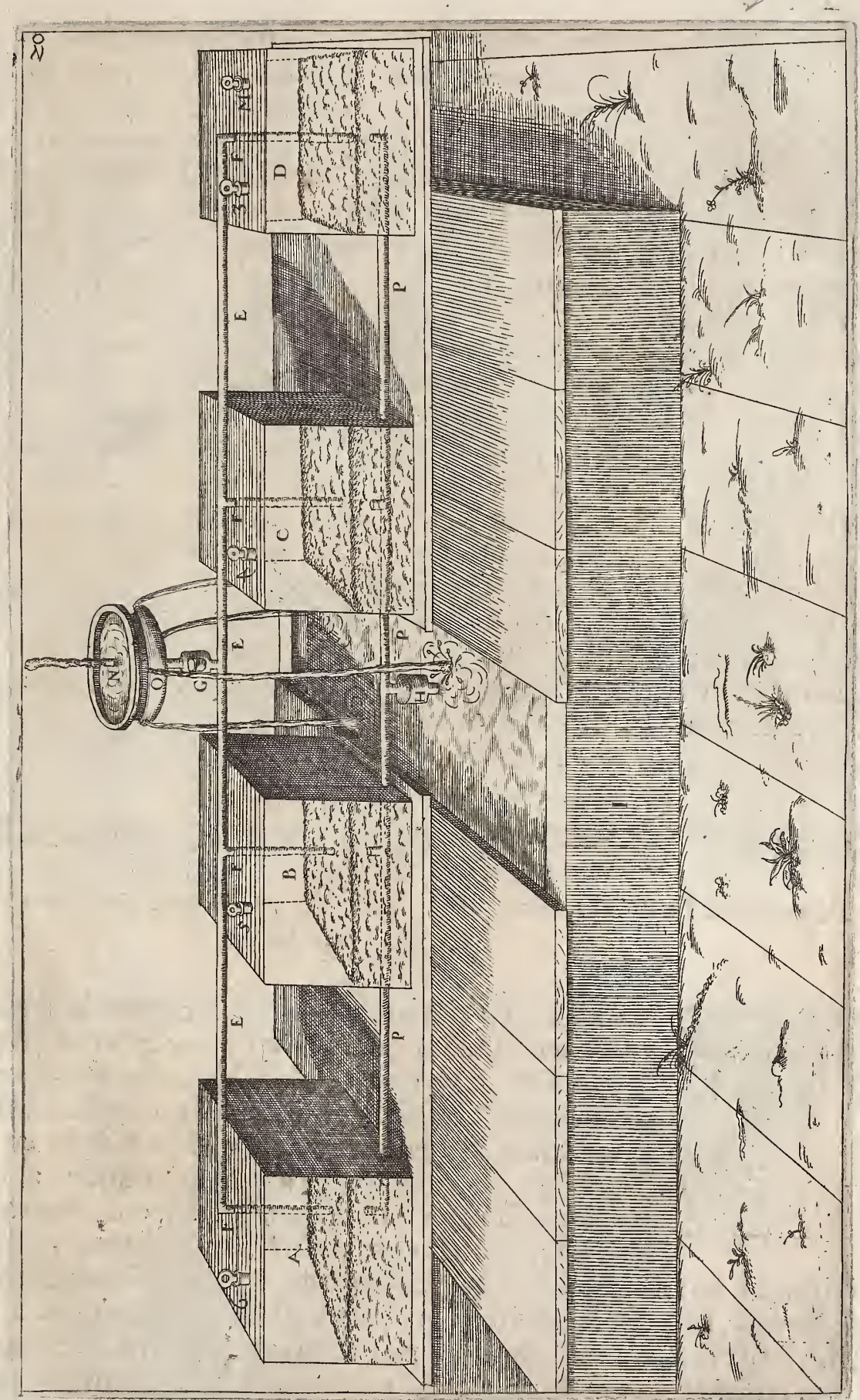




\section{Liure premier.}

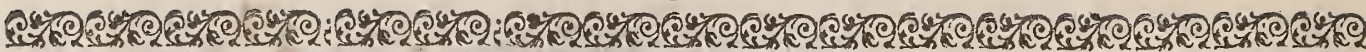

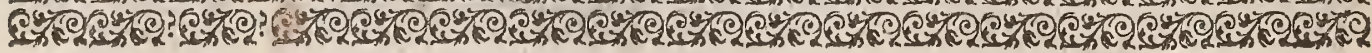

PROB L E S E X I III.

\section{OXachine par laquelle l'on augmentera la force de la precedente fontaine.}

\section{8} appellez miroirs ardans, lefquels teront bien aiultez dans le cuiure, en forte que l'air n'en puiffe fortir, lefdits verres feront marquez, les deux grands de chacun vaifeau, par les lettres C. D. \& l'es petits E. F. G. H. \& faut pofer le cofté du vaiffeau $\mathrm{L}$. vers le $\mathrm{Mydi}$, à celle fin que le Soleil donnant deffus lefdits verres ardans, raffemble les rayons du Soleil dans les vaiffeaux, ce qui caufera vne grande challeur à l'eau, \& par ce moyen forrira en plus grande abondance, \& aufli plus haut s'il en eft befoing, \& quand aux autres coftez des vaiffeaux ou font les verres, ils feront pofez vers l'Occident, pour eftre auffi le Soleil fort chaud apres Mydi, \& faut no. ter, que'fi la grande chaleur faifoit fortir toute l'eau qui feroit dans lefdits vaiffeaux, f̧̧auoir le tiers du contenu diceux, alors il en faudra mettre plus que le tiers, fçauoir la moitié du contenu defdits vaiffeaux, à f̧̧auoir par le foufpiral, comme a efté dit au precedent problefme, iay auffi fait cefte prefente figure plus grande que la precedente, \& quand à la foupape fuperieure, elle pourra eftre dansle vafe qui fouftiencle bafin de la fontaine. Et quand aux grandeurs des verresardans, ils pourront eftre comme ils font pourtraits aux figures. A. B. \& feront efpes par le milieu, comme lefdites figures le monfrent.

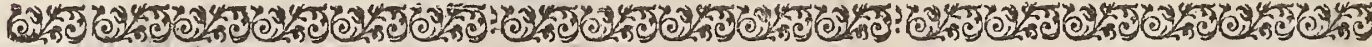

\section{Maniere de faire le ciment pour cimenter les verres aux vaiffeaux, en forte que lair nen puilfe fortir.}

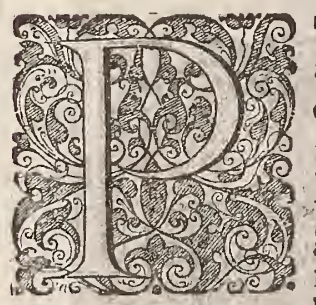

Ou r faire vn ciment bien durable contre la chaleur du Soleil, \& auffi quil puiffe bien prendre contre le verre, l'on prendra de la chaux viue, cinq ou fix pieces, lefquelles feront couuertes auec de tuille pulverifee, puis verfervn peu d'eau deffus ladit te tuille, laquel. le viendra à d'eftremper la chaux, \& la reduire en poudre, \& faut garder que ladite chaux ne foit trop humide, ains feulement mife en poudre, puis la deftremper auec du frommage mol, mellant auffi viron le tiers de ladite tuille battue, puis cimentter bien les iointures defdits verres auec les vaiffeaux de cuiure, il fe fait encores vne autre forte de ciment, lequel eft auffi tresbon, pour c'eft effect, à fçauoir du verre broyé auec de lhuylle de lin, \& mefler auffi vn peu de chaux defteinte, auec, ce dernier eft auffi tresbon contre l'eau, \& ne shumeete en aucune façon comme le premier, lequel fer. uira feulement pour les chofes qui font hors de l'eaa. 

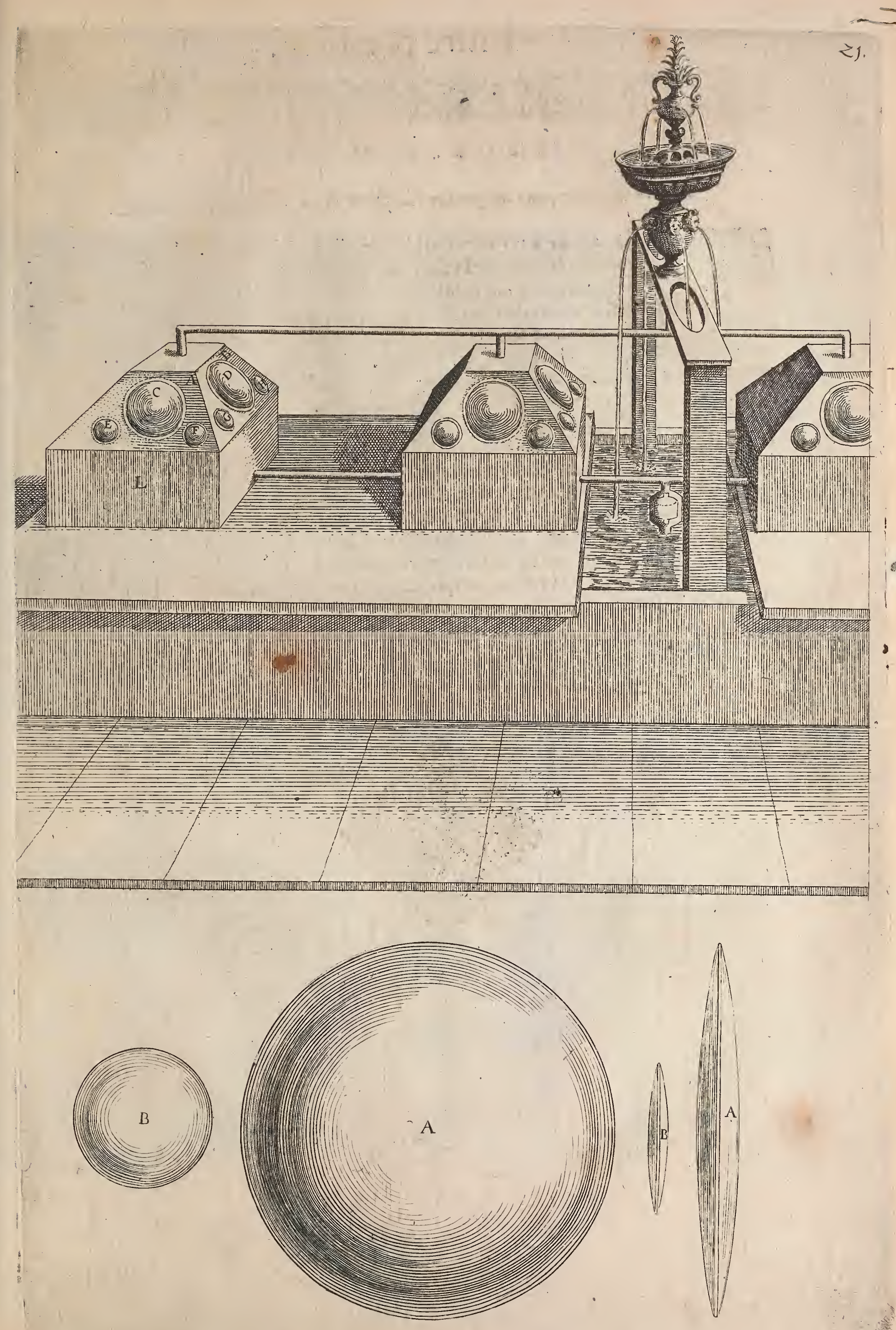


\section{Liure premier,}

W.

\section{P O B L E S M E X V.}

\section{Autre maniere pour augmenter la force de la fontaine preoedente.}

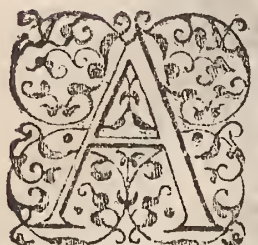

U PRECEDENT. Problefme, il a efté monfté le moyen daug. menter la force de la fontaine continuelle, \& dautant que les verres. ardans feront affez, difficilles à bien aiufter dans le cuiure, pour $\mathrm{cm}$.. pefcher l'air de fortir aux iointures, il ma femblé bon de demonftrer ence ter en la prefente figure, le chafo verres ardans, lis $A$. B. fera fait, en lorte que l'on puiffe enchafer quantité defdits verres ardans, lefquels feront pofez divne diftance de viron trois pieds, en forte que les pointes des cones ardans que produifene lefdits verres, puiffenc donner fur les vaifo feaux, lefquels eftans efchauffez par la violente chalcur defdits verres, fera monter l'eau en grande quantité, \& fera bon que ledit chafis foit grand, \& d'auoir plufieurs verres enchaffez, en iceluy, afin que le Soleil en faifant fon tour, quill y en aye tou. fiours quelques vns qui puiffent donner deffus less vaiffeaux, \& fi lon defire cacher lefdits vaiffeaux, en forte quills ne foyent veuës dans la chambre, l'on pourra faire vne perite galerie expofec vers le mydi, en forte que le Soleil puiffe donner fur leffits vaiffcaux qui feront dedans ladite galerie, puis paffer le tuyau C. D. dedans la muraille, \& conduire leau en la fontaine qui fera dedans la chambre, comme il fe fest voir en la figure. 


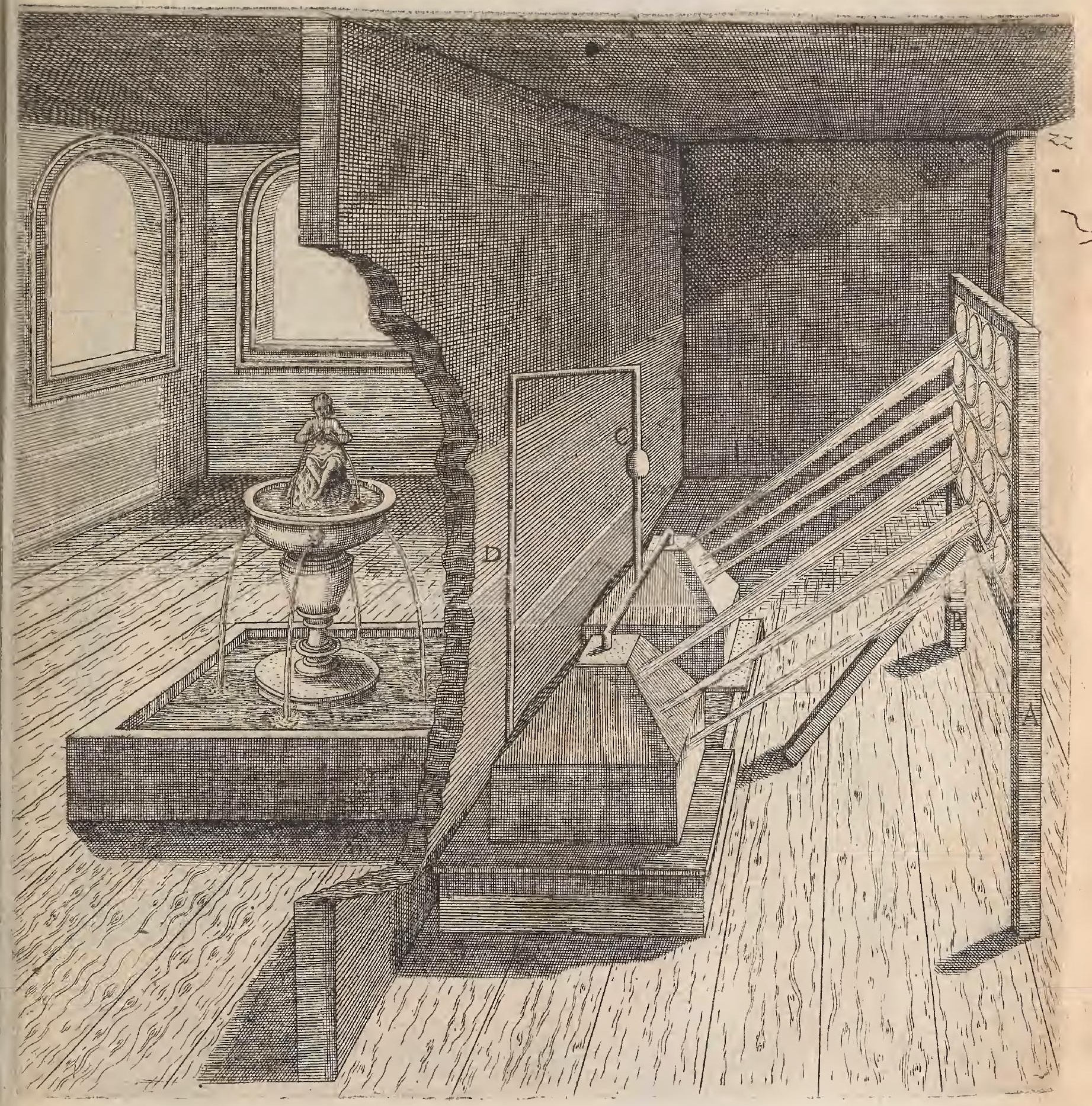




\section{Liure premier,}

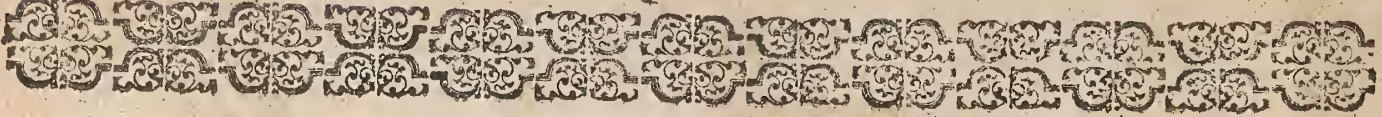

\section{P R O B LES M E X VII,}

\section{Orthographie de la precedente Mar}

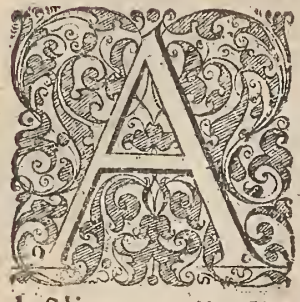

C $A$ SE que la precedente Machine eff fort diffcille à entendre, i'ay mis icy fon Orthographie, on il fe peut voir comme les deux roués longues $G$. $H$. fe hauffent par le moyen du pionon $X$. Si ce mpumement eft bien entenda, il pourra feruir en plufieurs autres chofes diuerfes, comme à farre tirer des Sies, pour fierdubois, tres moutuements, lefquels ont befoing de hauffer \& baiffer, lefdites rouess $\mathrm{H}$. \& refque perpendiculairement. Il faut auff noter, que tant plus droit mais deutant que le papier le peur permetre rie faut, pour eftre bien, \& aufi il n'eft pantraint de faire les pieces plus courres quil

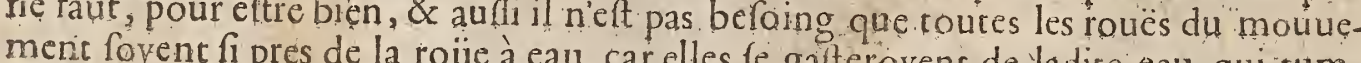
ment foyent fi pres de la roüe à eau, car elles fe gaiteroyenc de lạdice eau qui tum. beroit deflus, mais quiconque voudra faire ledit mouuement on les metra va peu plus loing.

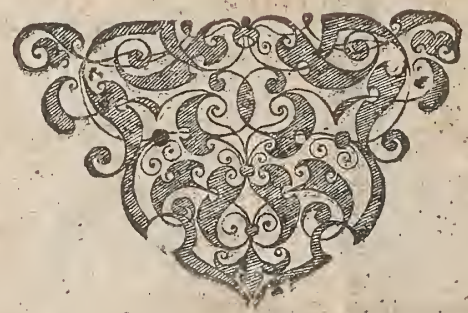



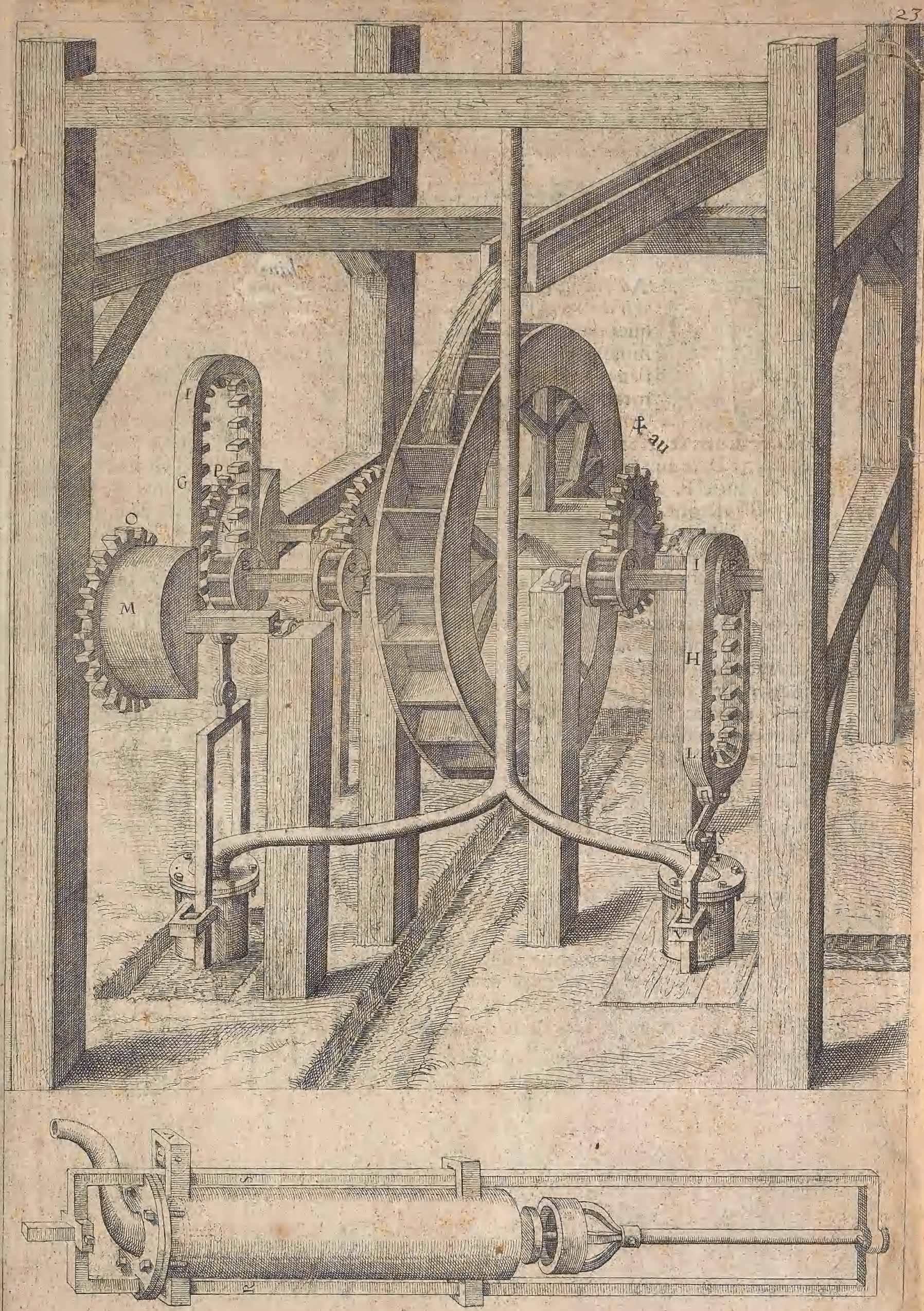


\title{
Livre premier,
}

4\% 5\%

\section{PROB LES ME XVI.}

\author{
Tour faire monter l'eau par le moyen des pompes, EG d'vne roue à eau.
}

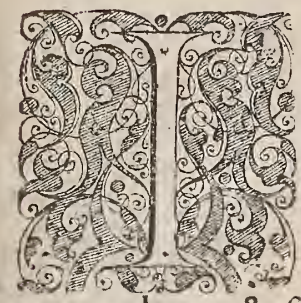

Ax enfeigné par cy deuant, aux trois premiers Problefnes, le mo. yen d'efleuer l'eau par le moyen des Pompes, \& pour donner quelques varietez des deffeings, i’ay encores mis ceftuy.cy, lequel a fon mouuement auec quelques rouës dentelees, fort propres pour eneuer \& abaiffer les feaux defdites pompes, foir donques premiement la rouë à eau à laxe, de laquelle fera deux roues dentelees, marquez l'vne A. \& lautre B. lefquelles auront chafcune vingt \& quatre dents, \& feront tourner chacune vn pignon de fix dents, marquees l'vn C. \& l'autre D. \& aux axes defdits pignons, feront deux autres pignons, l'vn marqué E. \& l'autre $F$. apres lion aura des roues, comme il fe peut voir aux figures G. H. lefquelles feront faites prefque en oualle, mais les coltez teront tous droits depuis $L$. iufques à I. en forte que les pignons $E_{\text {. }} \& \mathrm{D}$. cournans, puiffent leuer lefdites rouës perpendiculaires, \& quand ellẹs feront leuees, (comme lefdits pignons tourneront toufiours) fera que lefdites rouës longues, iront vn peu de cofté, iufques à ce que les autres branches droites de derriere fe viennent à rencontrer contre lefdits pignons, \& alors lefdites roües, fi elles eftoyent hautes fe rabaifferont tout droit, ainfi lefdites rouès hauffants \& abaiffants, feront leuer $\&$ abaiffer les feaux. Or d'autant quelefdites rouës ne tournent point, \& quelles ne font que hauffer \& abaiffer, il fera befoing pour les faire tenir en eftat contre lefdits pignons, de faire que deux autres roués marquees $O$. P. feront difpofees, en forte qu'vn femblable pignon les tournants toutes deux, feront en forte que deux demies tambours marquez M. N. rournants tantoft l'vn d'vn colté, tantolt de l'autre, fera caufe de faire tenir lefdires rouës G. \& H. en eftar, \& pour faire meilleure demonftration de la figure, ie n'ay poine mis lefdites rouës $M$. N. d'vn cofté, \& aufit que pour plus facille intelligence de ce mouuement, i’ay mis le plan de l'ortographie fuiuant, \& aufli au bas de ce prefent mouuement, i'ay mis, vne des pompes en plus grand volume que non pas au deffeing, \& faut noter, que quand leldices rouës longues fon pouffees de cofté, quand elles font en haut, alors en defcendant, elles ne pouffent pas les branches des pompes perpendiculairement, comme il fe peur voir au cofté $H$, \& à celle fin que par ce defaut lefdices branches ne laiffent de defcendre droit, \& fańs eitreforcees, I'on metra vne petite roue de cuiure marquee $T$. dans la charniere $V$, il faudra auti noter, que le pignon qui fait mouuoir les rouc̈s $O$. \& $P$. ne fepeut voir, mais il le faut confiderer eftre paff̌c au mefme axe de ceux E. \& C. \& auffi il faudra que lefdites roües O. \& P. contiennent chacune autant de dents comme les longues roües. 


\section{Liure premier,}

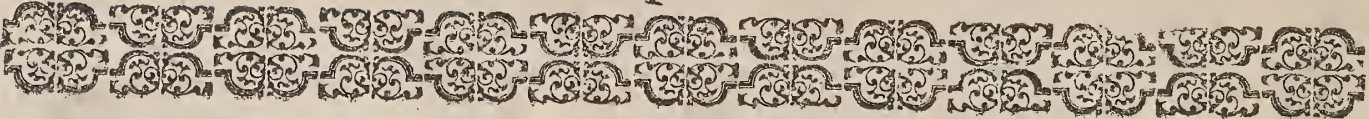

\section{PROBLES ME XVII, \\ Orthographie de la precedente Machina.}

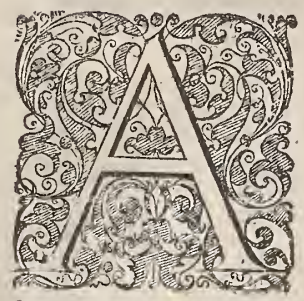

CA U s Eque la precedente Machine eft fort difficille à entendre, i’ay mis icy fon Orthographie, ou il fe peut voir comme les deux roués longues G. H. Re hauffent par le moyen du pignon X. Si ce mouuement eft bien entenda, il pourra feruir en plufieurs autres chofes diuerfes, comme à faire tirer des Sies, pour fier dubois, $\&$ autres mouuements, lefquels ont befoing de hauffer \& baiffer, lefdites rouës H. \& refue perpendiculairement. Il faut auffi noter, que tant plus droit, mais dautant que ie faits au deffeing les pies, tant plus le mouuement va que le papier le peut permettre, ie fuis contraint de au mouement aufí grandes re faut, pour eftre bien. \& aufil neft pas befoing queces plus courtes qu'il ment foyent ment foyent fi pres de la roüe à eau, car elles fe gafteroyent de ladite eau qui tumberoit deffus, mais quiconque vaudra faire ledit mouuement on les mettra vo peu. plus loing. 


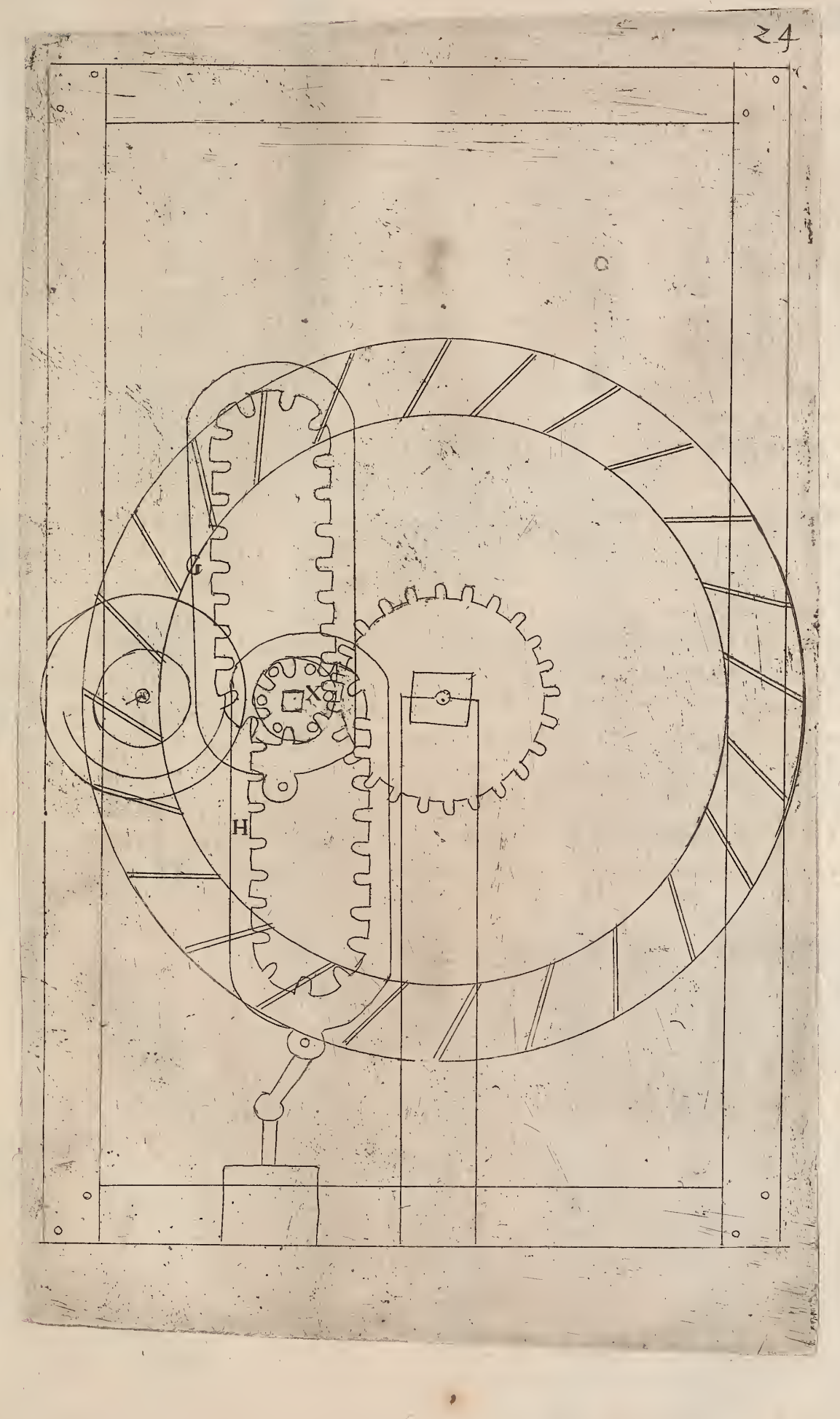




\section{Liure premier,}

(อ)

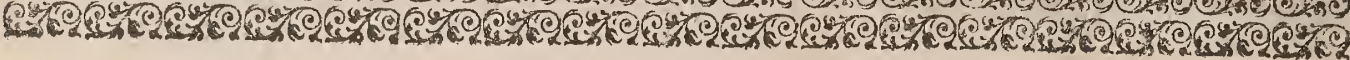

\section{PROB L E S E X V I I I.}

\section{OCachine par laquelle l'on pourra par la force d'vne roue à cau, faire fier du bois, auec grande promptitude.}

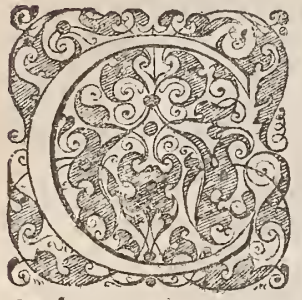

Este Machine eft fort commune entre les montaignes au pays des Suiftes, auec laquelle ils font fier grande quantice de planches de Sapin, ladice Machine eft fort neceffaire deftre en vne grande ville, ou dans vne foreft où l'on fait fier du bois, foit en planches ou en autres formes, celle icy neft pas du tour femblable à celles defdits Suilfes, car ils font aprocher la piece de bois des fres, par le moyen de quelque roues denrelees, auec vn roquet, mais a cau. fe des reparations qui viennent fouuent audices roues dentelees, ie tache coufiours d'en euiter l'vage autant comme ie peux, ainfi i'ay mis les deux contrepois de viron deux ou trois cents liures chacun, done l'vn eft marqué A. \& l'autre fe doit imaginer au bout de la corde B. (car s'il cut efté deffeigné, il empefcheroit la veúedu mouuement de la charniere C. par laquelle les fies hauffent $\&$ abaiffent perper. diculairement) les cordes ou pendent lefdits contrepoix, feront attachees rout au der. riere defes déux pieces debois mobiles, lefquelles gliffent fur deux autres pieces de bois ftabilles, par le moyen de quelques petites poulies qui pourront eftre dedans la charniere, \& ainfi lefdits contrepois tireront toufrours lefdites pieces de bois mobilles, \& la piece que lon defire eftre fiee, fera ferme entre lefdites pieces mobiles, laquelle auançant toufiours auant, \& lẹs fieshauffans \& baiffants, pourront fier ladite piece en gräde diligence, l'on pourra mettre deux trois ou quatre fies au plus fur le fuft, diftantes l'vne de l'autre, autant comme lon veut auoir d'epeffeur aux planches, \& quand la piece de bois fera au bout, alors vn homme ou deux auec vn leuier tourneront vn rouleau, ou fera attachee vne forte corde, qui fera reuenir ladite piece en arriere, \& rehauffer les contrepois, \& apres on mettra ladite piece de bois vn peu de cofté, pour faire reprendre les fies derechef contre ladie piece de bois.

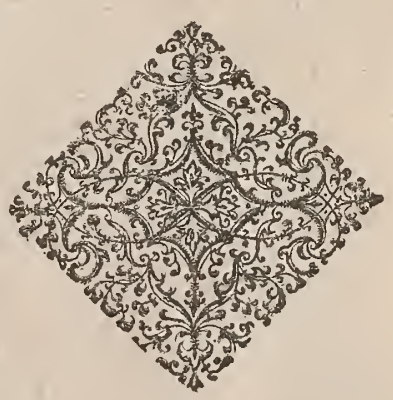




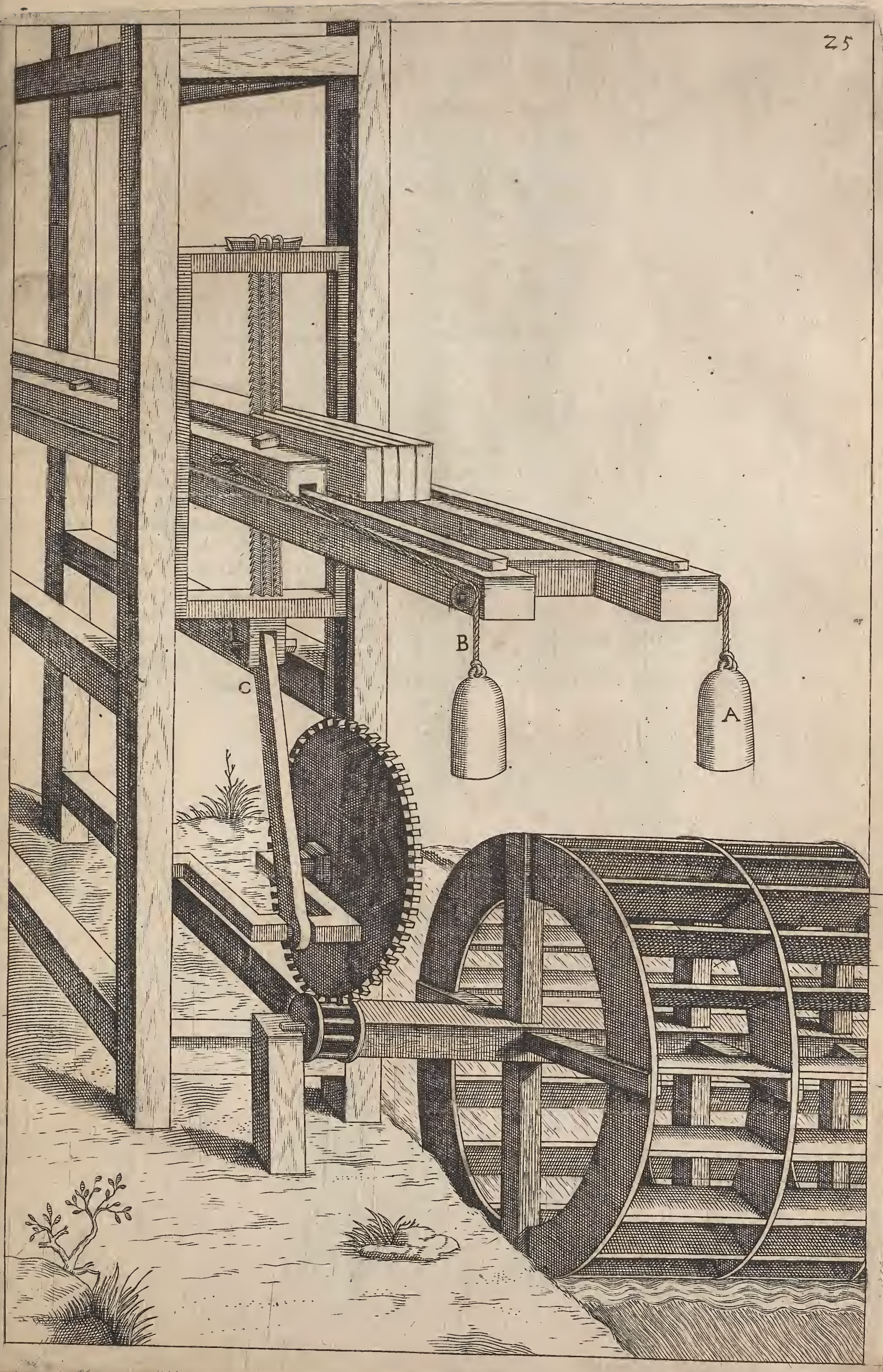




\section{Livre premier,}

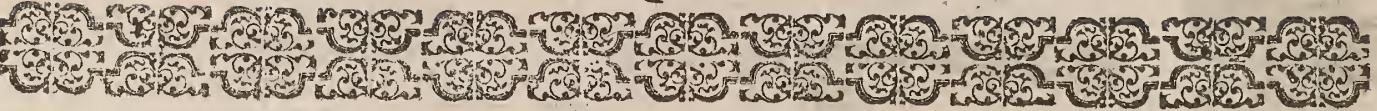

PROBLES ME XIX.

evacbing de grand fervica, propre pour percer des pipes de bois.

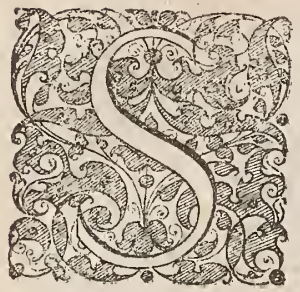

Ort vne rouè à eau, à laxe de laquelle fera vne rouë dentelee de trente \& fix dents, ou dauantage, felon la viteffe de la roüe à eau, car fi elle taurne lentement, il en faudra d'auantage, \& y aura vn pignon de fix dents, que ladite roüe dentelee tournera, comme il Ce.peut voir en la figure, \& à laxe dudit pignon fera ioint vne longue tarelle marquee $A$. laquelle fera pofee à trauers vn trou marqué B. souurant \& ferrant comme la lunette d'vn tour à tourner, apres l'on pofera la piece de bois (pour percer) ferme fur vn chantier marqué C. D. en forre que ledit chantier puiffe gliffer facillement par le moyen de quelques perites roues, lefquelles ferant dans la graueure d'iceluy, \& courneront fur la charniere ftabla, en forte qu'vn hom me puiffe auec fa force, pouffer \& retirer ladite pipe quand elle fera ferme fur ledit chancier, \& ainfi la tarelle tournant, l'homme pouffera le bout de ladite piece de bois contre, \& apres que ladite tarelle aura percé deux ou. trois pauces auant, il faudra incontinene retirer ladite piece de bois arriere, à celle fin de faire vuigder le bois de la tarelle autrement elle feroir en danger de rompre, \& faudra continuer roufiours de retirer ladite piece, quand elle aura percé trois ou quaue pouces, pour vuider toufrours ledic bois, iufques à ce que le trou foit outre, \& a. pres fi l'on veut ledit trou plus grand, l'on prendra vne certaine façon de tarelle comme la figure $\mathrm{E}$, le monftre, laquelle eft faite prefque comme vne cuillier taillante par les bors, \& en paffant ladite tarelle agrandira fort le trou ia fait.

PRO. 


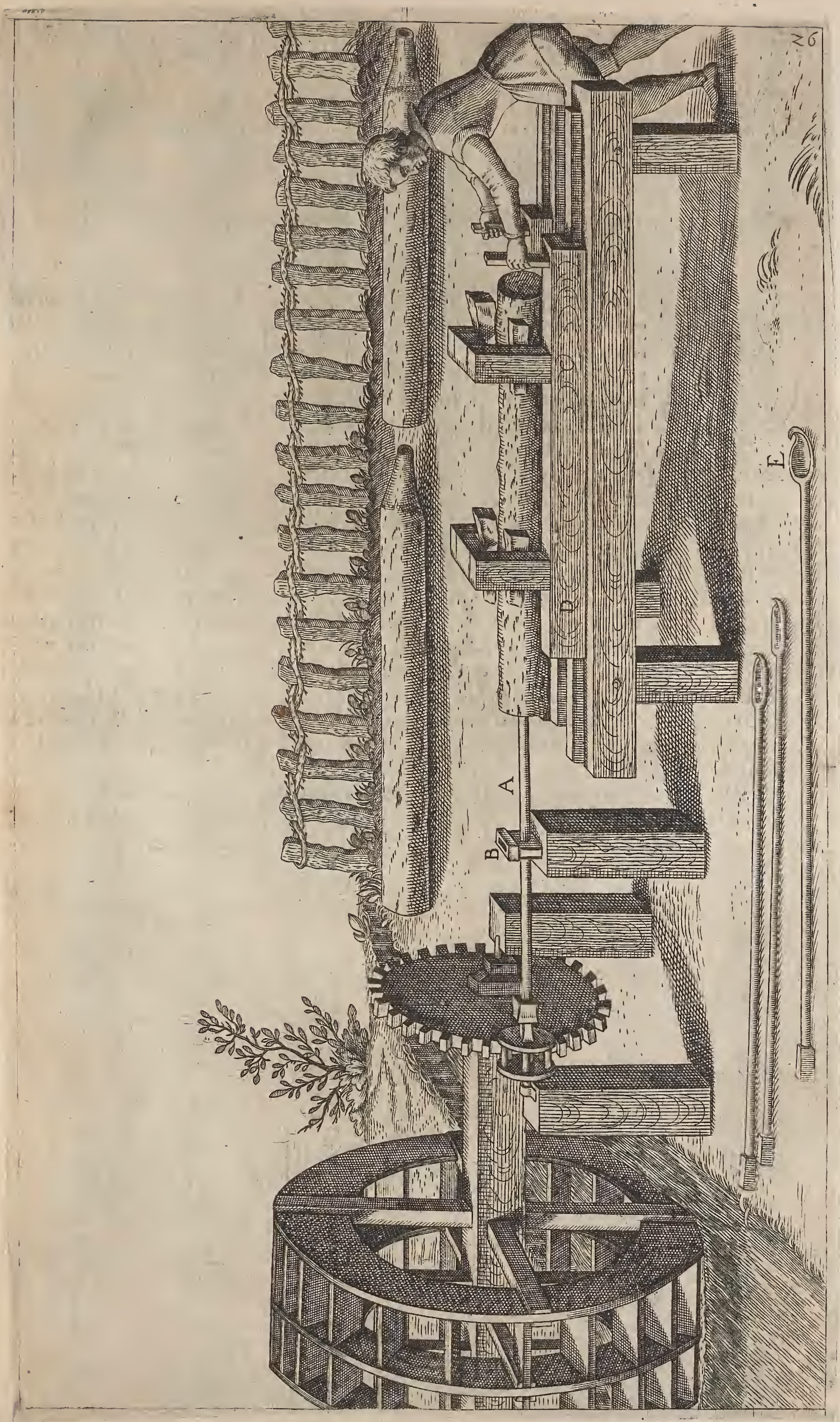




\title{
Liure premier,
}

10.

\author{
P R O B L E S M E X X.
}

\section{OXachine fort neceffaire par laquelle lion peut donner grand fecours aux maifons qui jeroyent enflambees.}

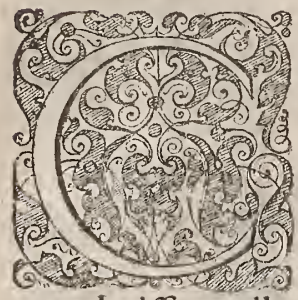

Esтв machine eft fort experimentee en Alemaigne \& ay veu le grand \& prompt fecours quelle peut aporter, car encores que le feu fut 40. pieds haut, ladite machine y iettera fon eau par le moyen de quatre ou cinq perfonnes qui hauferont \& abaifferont vne longue branche en forme de leuier, ou la branche de la pompe eft atachee, ladite pompe eft facille à entendre, par dedans il y a deux foupapes, vne en bas pour ouurir quand lon hauffe la branche, \& en rabaiffant elle ferre, \& vne autre ouure pour laiffer fortir leau, $\&$ au bout de ladi. te machine, il y aura vn homme, lequel tiendra la pipe de cuiure $A$. la tournane d'vn cofté \& dautre, fuyuant le lieu ou le feu fera, quand on veut hauffer ou abaiffer ledit tuyau, fe fera par le moyen d'vn autre tuyau ioingnant, marqué B. \& faut que lefdits tuyaux l'vn mouue d'vn cofté, \& d'autre, à celle fin que l'on puiffe tourner, hauffer \& baiffer ledit bouc $A$. fuiuant l'occafion, \& d'autant que l'eau qui eft recuesllie fur la roue eft pleine d'ordures, \& que facillement les foupapes pourroyent eftre empechees de ferrer par icelles, pour cefte occafion à la cuue dans quoy l'on verfe l'eau, il y aura vne treille au millieu, de trous menus comme vne bien groffe efplin. gle, \& ferois dadvis (veu la grande utilité que cefte machine peut aporter au befoing, \& le peu de couft d'icelle) qua chacune paroiffe de ville', il y en eut vne, laquelle à vn befoing fe peut trainer par trois ou quatre hommes ou le feu pourroit eftre, \& alors mettant de l'eau dans la cuue, elle eft pouffee en haut, fans peril d'hommes n'y atirail, d'efchelles, \& faut noter, que fi les foupapes font de curr, (comme l'on vfe en beaucoup de licux) alors il fera befoing que ladite cuue foit toufiours plaine d'eau, autrement ledit cuir venant à fe fecher, feroit manquer la machine au befoing.

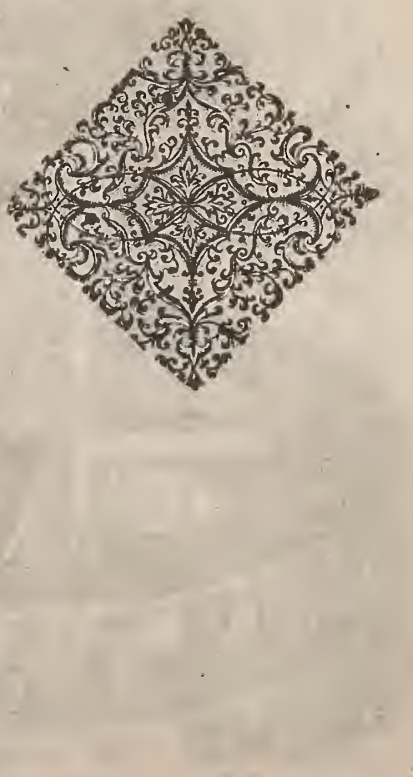




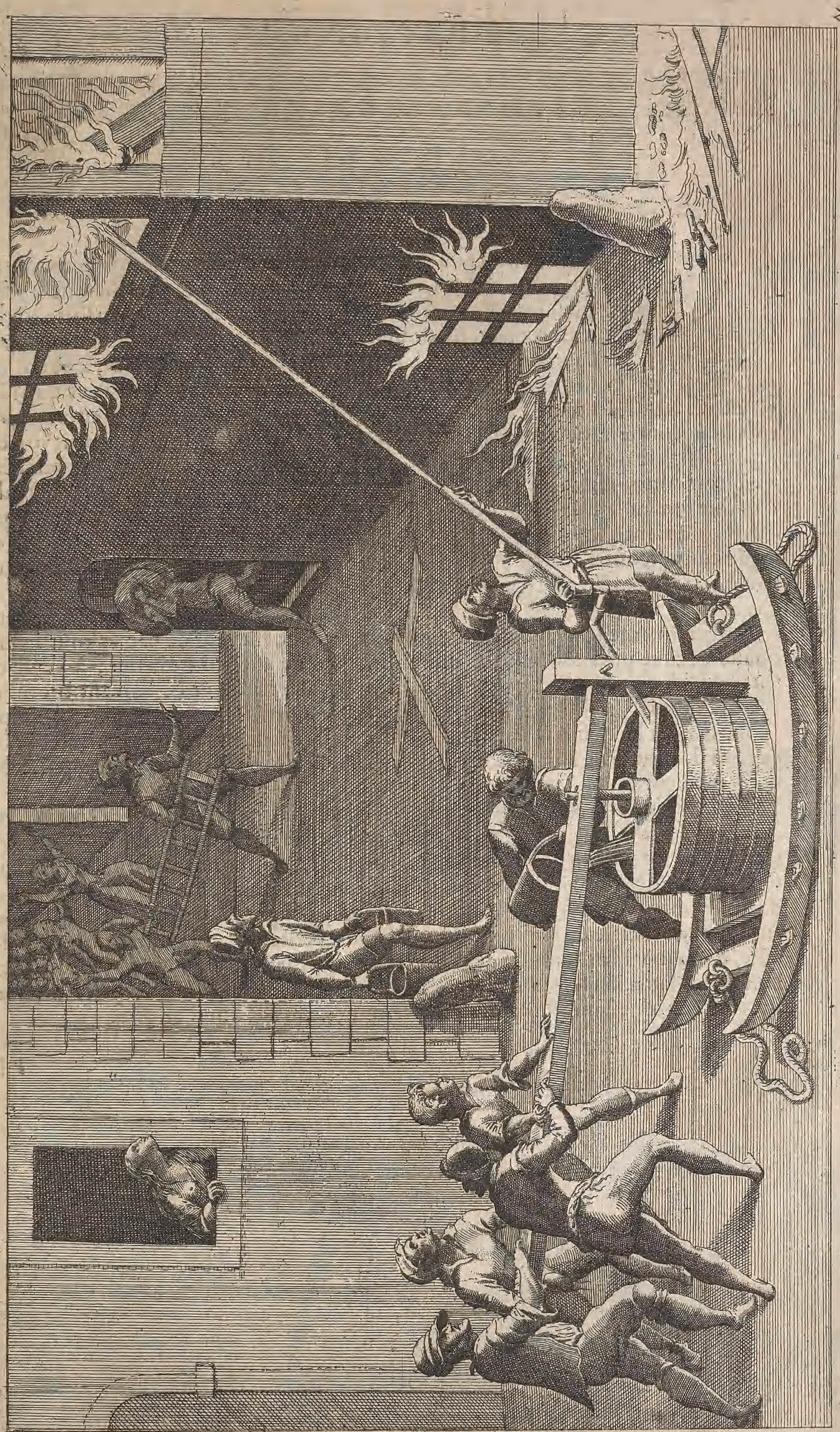




\title{
Liure premier,
}

2.

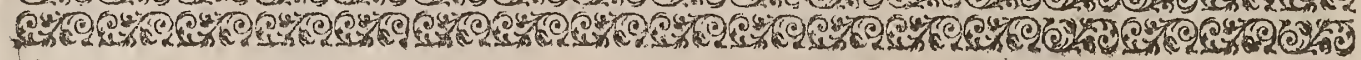

\section{PROBLES M E XXI.}

\section{Grachine fort jubiille pour taumer en onalle quelque chofe que ce foit.}

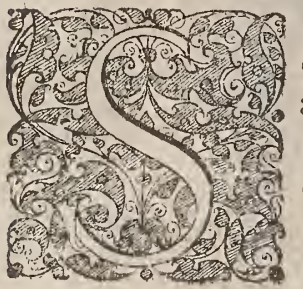

OI v v trou paur tourner, fait comme la figure fuperieure monftre, ou feroncaiuftées deux pieces de fer marquées B. A. lefquelles feront atachées contre les deux gros traineaus de bois C.D.en farte quelefdi. tes pieces foient mobilles de cofté \& dautre, \& ferontatachées chacuneauec unecorde ou il y aura vn contrepois pendu au bout comme il fe peut voir en la figure de bas, apres l'on paffera deux oualles de cuiure perires à trauers vin axe de fer marquées E. F. en fortequ'elles couchent concreles denx gardes ferres de fer marquées G. I. commeil fe peut voir encores en la figure de bas, \& ainfi quand laxe de fer rournera, alors lefdites oualles qui touchent contre lęs guardes ferres fermes, cauferont ladi te axe de varier çà \& là, de ta. çon que tenant le fer ferme contre louurage que l'on defire faire, fera ledit ouurage sualle, d'autantque ladite axe tourne en oualle a caufe de la variation despetites oualIes de cuiure conere les gardes ferres, \& au millieu de ladie axe de fer il y aura vne poullie de bois ou fera palfée vne grofle corde de boyau, laquelle fera auff paffée dans vne autre grande roüe qui fera taurnée par vn garçon, \& ainfi en tournane touf: iours d'vin mefme fens, l'on trauaillera fort facillement ${ }^{2}$, car de penfer tourner ladite oualle auec le pied comme au tour ordinaire il ny a aucun moyen à caufe de la force qui eft trop grande, \& aufi que ladire aualle hauffant \& baiffane brouilleroit louril, duquel on fe fert a courner, il y en a aucuns lefquels au lieu de contrepords (pour bender les oualles de cuiure contre les guardes ferres) fe feruent de reffors dafcier, mais à caufe de linefógulle force defdits refforts ie trouue les contrepaids beaucoup meilleurs.

Il faut icy noter que le deffein de bas of femblable à celuy de haur, mais l'vn eft toumé d'vn cofté \& l'autre de lautre, \& céla à efté fait pour mieux confiderer les effects/des guardes ferres, \& des contrepo ds, il faut aufficonfiderer que lefdites gardes ferres entrent \& forcent dehors aulfi auant que l'on veut, \& s'arreftent par le moyen d'vne petite vis mąrquée $H$. car il eft befoing quelquefois de changer les oualles de cuiure \& en mettre de plus grandes ou de plus petites felon tou faire, \& quand à la pefanteur des contrepoids, ils feront auff fuiuant louurage quie lon defre faire, carf louurage eft petit, lefdits contrepoids pourrontpeferis. pu zo. li. ures chacun, \& eftant plus grand ils peferont 5o. ou foo, liuręs au plus.
\end{abstract}




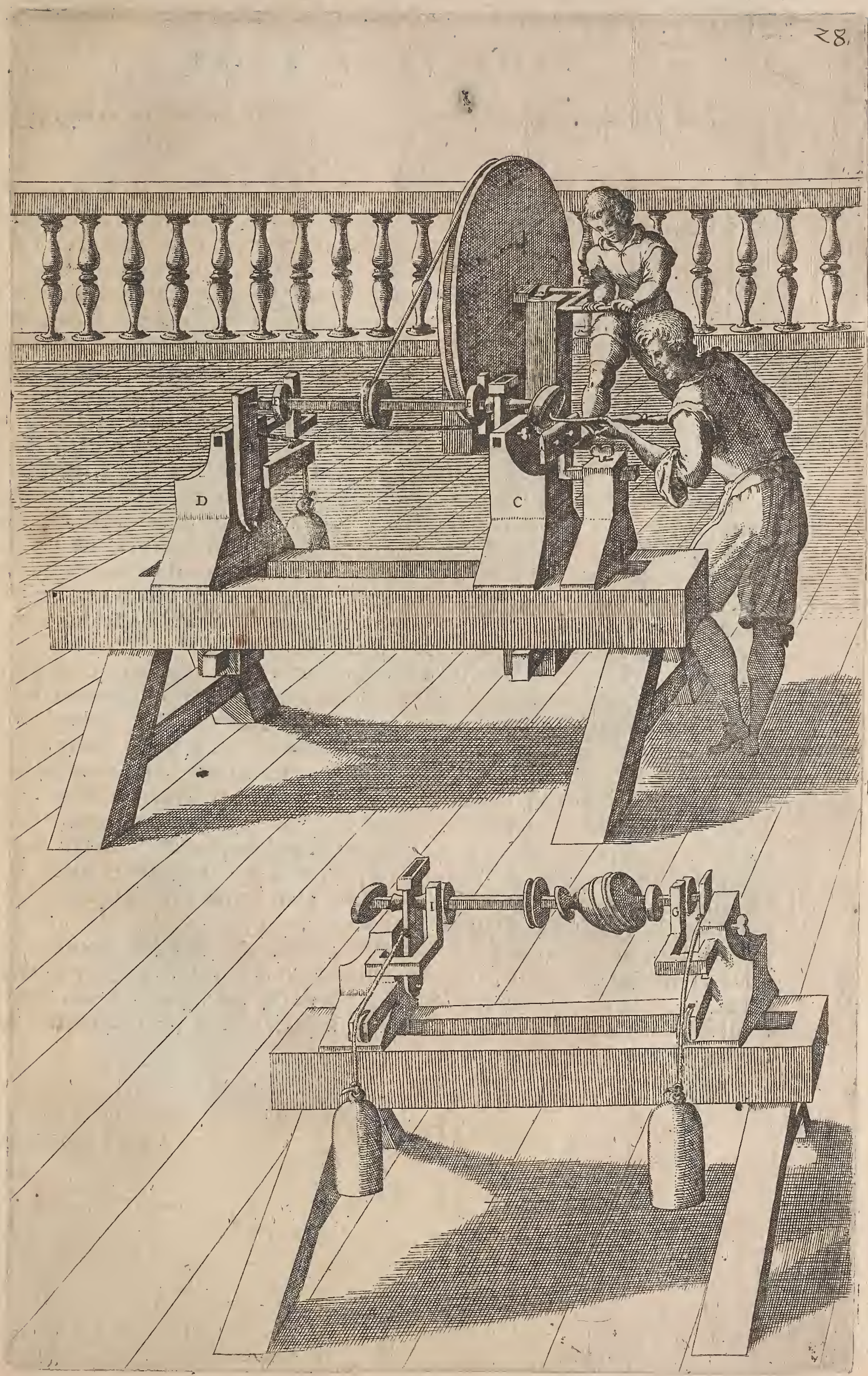




\section{Liure premier,}

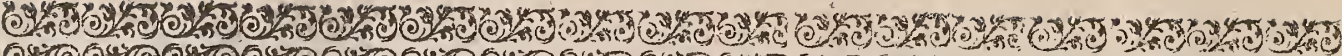

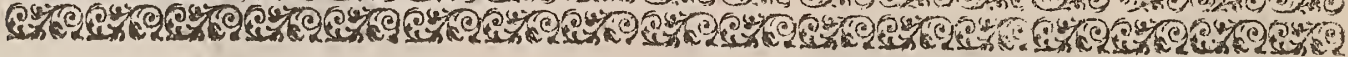

\section{PROBLES ME XXII. \\ Pour faire reprefenter le chant d'vn oy feau en fon naturel, par le moyen de leaur.}

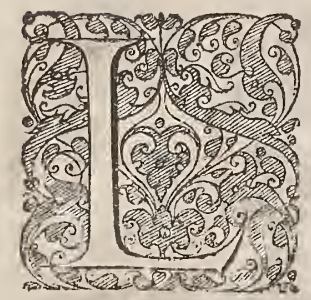

E CH A $\mathrm{T}$ ou ramage du rofignol eft affez dificile à le bien reprefenter en fon naturel, tourefois ie donneray icy voe inuention, laquelle imitera de bien pres ledit chant, foit premierement deux vaifeaux marquez A. \& B. \& foir auffi vn tuyau foudé contre le fond A. auquel ily aura vn robinet marqué Q.lequel fervira pour donner leau \& faire tourner la roüe $T$. laquelle fera de viron deux pieds en diametre,  roue, il y aura vn pignon de huir dents, marqué C. apres faudra auoir vn petit tabourin marqué $E$. de viron huit ou dix pouces en diametre lequel fera bien arondi, \& au cofté $D$. Il y aura un petit receptacle pour receuoir l'eau qui tombe fur la roue $T$. \& audit receptacle il y aura vn tuyau marqué $P$. allant iufques pres dúfond du vaiffeau $B$. \& en la fuperficie dudit vaiffeau il y aura deux tuyaux marquez $L$. M. aufquels feront foudez deux robinets \& à chafcun diceux, il y aura vne reigle de cuiure foudee à chacune clef defdits robinets, en forte que quand l'on abaiflera les bouts V. X. defdites reigles, les robinets fe puiffent ouurir, apres faudra metre des cheuilles fur le tabourin marquees $\mathrm{F}$. G. lefquelles abaifferone les bouts defdites reigles, \& feront osurir lefdits robinets, à celle fin que l'air du vaiffeau de bas monte par les tuyaux L. M. \& fe rende à deux fifflets, qui féront au bout defdits ruyaux, \& fi l'on veut reprefenter le chant d'vn Roffignol, lon mettra trois ou quatre cheuilles fuiuantes l'vne l'autre pour toucher fur vne merme reigle, auec quelque peu dinterualles entre icelles, puis l'on mettra vne longue touche ou cheuille pour tenir l'autre reigle baffe, le refte de l'efpace du peric tabourin, \& au bout defdits porteuents $L$. $M$. (comme a efté dit) il y aura deux fiffers à vn dicon l'vn de l'aurre, fauoir celuy qui fonnera trois ou quatre fois fera le plus bas; \& l'autre laigu, \& les bouts defdits tuyaux tremperont dans leau, comme en la precedente, mais fi lon veut reprefenterlechant du Coqu, il y aura feulement deux touches delfus le tabourin, comme il fe peur voir en la figure, \& lés fifflets feront de la groffeur d'vin pouce \& demi en diametre, \& le plus long aura vn pied, \& l'autre dix pouces, \& feront couchees aupres de l.oy-. feau, comme il fe peut voir en la figure H. I. mais fi l'on veut reprefenter le chant d'vn Coq, l'on metrra au bout des'porteuents des tuyaux appellez des faifeurs dorgues tuyaux à anchès, ou regalles, accommodant les cheuilles du tabourin à propos pour reprefenter ledit chant, il faudra auffi attacher des fillers de cuiure pres desbouts des reigles, pour en abaiffant faire ou urir le bec de loyfeau, quand lefdirs bouts des reigles s'abaifferont, \& quand au mouuement dudit oyfeau il fera reprefente au problefme fuiuant. 


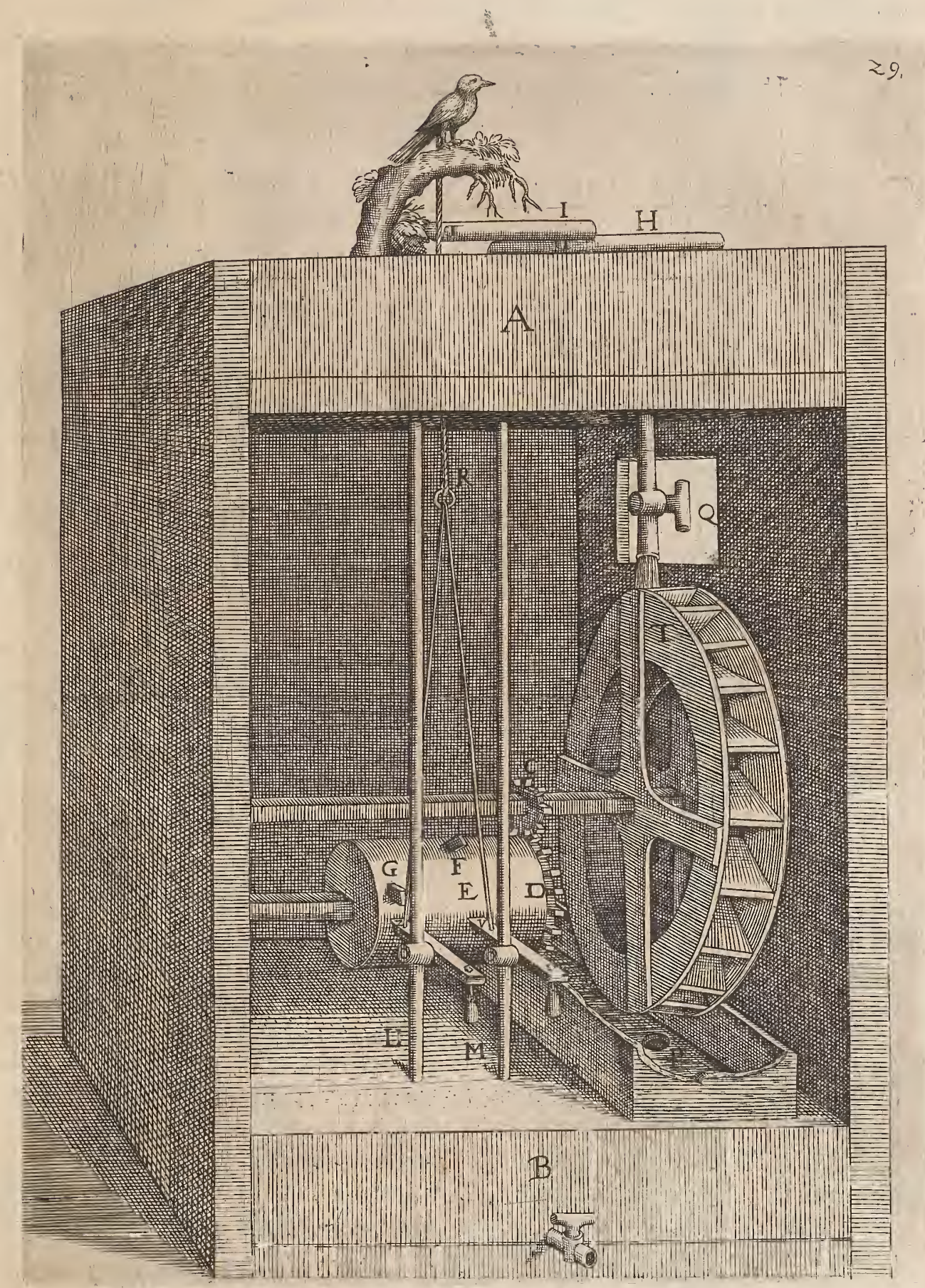




\section{Liure premier,}

$3 \times$. 3 .

P R O BLES ME X XIII.

\section{Pour faire reprefenter plufiesurs oifeaux le fquels chanteront diuer fement quand vne choüet- te (e tournera vers iceux, Eq quand la dite choüette (e retour- nera, ils cefferont dechanter.}

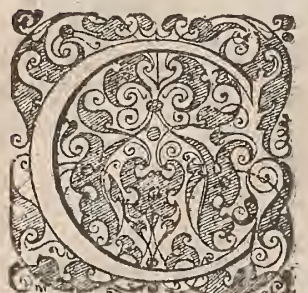

E mouuement à efté autrefois reprefenté par Herone Alexandrin, mais non auec fi grande varieté d'oy feaux comme ie le reprefenteray icy, foit donques comme en la precedente vne roüe à cau A. laquelle tournera dans vne caffe de plomb où cuiure marquée $C_{\text {. }}$ laquelle caffe feruira pour empefcher que leau qui tombe fur la

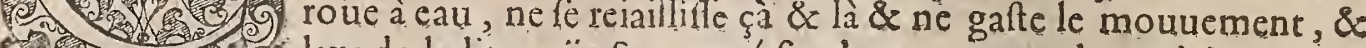
cofez de ladice roüe fera apuyé fur deux trous ronds, qui feront aux coftez de ladite caffe, \& à l'vn des bouts dudit axe qui fortira hors de ladite caffe, il y aura vn pignon de 8 . dents marqué $D$. lequel fera tourner vn tabourin commeen la figure precedente, mais ledit tabourin fera vn peu plus grand, fçauoir de 12. ou Is. pouces en diamettre, \& auffi il tournera de l'autre fens, fçauoir au lieu qu'en la pre. cedente les bouts des reigles font abaiffées pour faire ouurir les robinets, ceftuy cy les hauffe, non qu'il foit neceffaire que cefte diuerfité foir, mais cela eft fait pour don. ner à choifir des deux façons, apres il y aura trois porteuents marquées E. F. G. aufquels feront foudées les 3 , robinets H. I. K. \& aux clefs defdits robinets feront fou. dées les 3. reigles comme en la precedente, en forte que quand les cheuilles leuent les bouts defdites reigles N.O. (comme apert en la figure) lefdits robinets fe puiffent ouurir, \& au bout de haut du porteuenc $\mathrm{E}_{\text {. }}$ il y aura deux où trois fiftets, pour re. prefenter le chant des roflignols \& autres petits oifeaux, \& aux deux autres porteuents F. G. il y aura aux bouts de haut diceux deux fiflets de mefme mefure, comme en la precedente, pour reprefenter vn coucou, \& quand aux cheuilles pour hauffer les reigles, celle $P$. contiendra les $\frac{3}{4}$ de la circonference du tabourin, à celle fin de tenir le robinet $N$. long temps ouuert, \& les deux autres cheuilles $Q$. R. feront courtes en forte que quand $R$. fera paffée, \& quelle aura fait ouurir le robinet $K$. lau. tre marquée Q. fe prefentera \& fera ouurir I. \& fes deux reprefenteront le chant du coucou par le moyen des deux fiflets qui font aux bouts des porteuents G. F. apres la choüette fera pofée à l'autre cofté du mouuement, comme la figure demonitre deffus vn petit bloc, auquel fera paffé vn petic axe de fer qui refpondra à traucrs la pou. lie marquée $S$. \& fera ferme à ladite poulie, en forte que quand on la tourne, que la chouette fe puiffe tourner affi, il y aura femblablement deux autres poulies mar. quées V. T. aufquelles fera paffée vne corde à l'vn des bouts de laquelle il y aura vn vaiffeau tresbuchant $X$. \& l'autre bour fera ataché à vn perit leuier marqué 3.4 . \& au bout marqué 4 . dudit leuier, il y aura vn petit contrepois, lequel fera balancé auec le vaiffeau $X$. en forte que quand ledit vaiffeau fera à demy plain d'eau, qu'il puiffe atirer le bout dudit leuier auec le contrepois en haut, \& au contraire, quand ledit vaiffeau fera vuidé, que ledit bout auec le contrepais puiffe atirer ledit vaiffeau en haut, il y aura auffi vne cheuille marquée 6 . ferme contre le fond du tabourin, pour arrefter ledir tabourin, par le moyen de la reigle 3. 4. \& auffi il y aura deux tuyaux, lefquels donneront l'eau, fçauoir celuy B. fur la rouë à eau, \& celuy Z. (dont le bout fera menu comme le tuyau d'vne plume à efcrire) donnera dans le vaiffeau $X$. tellement que quand ledit vaiffeau fera à demy plain, il s'abaiffera \& fera leuer le bout du leuier 4 . alors la rouë à cau qui ne pouuoit tourner auparauane 


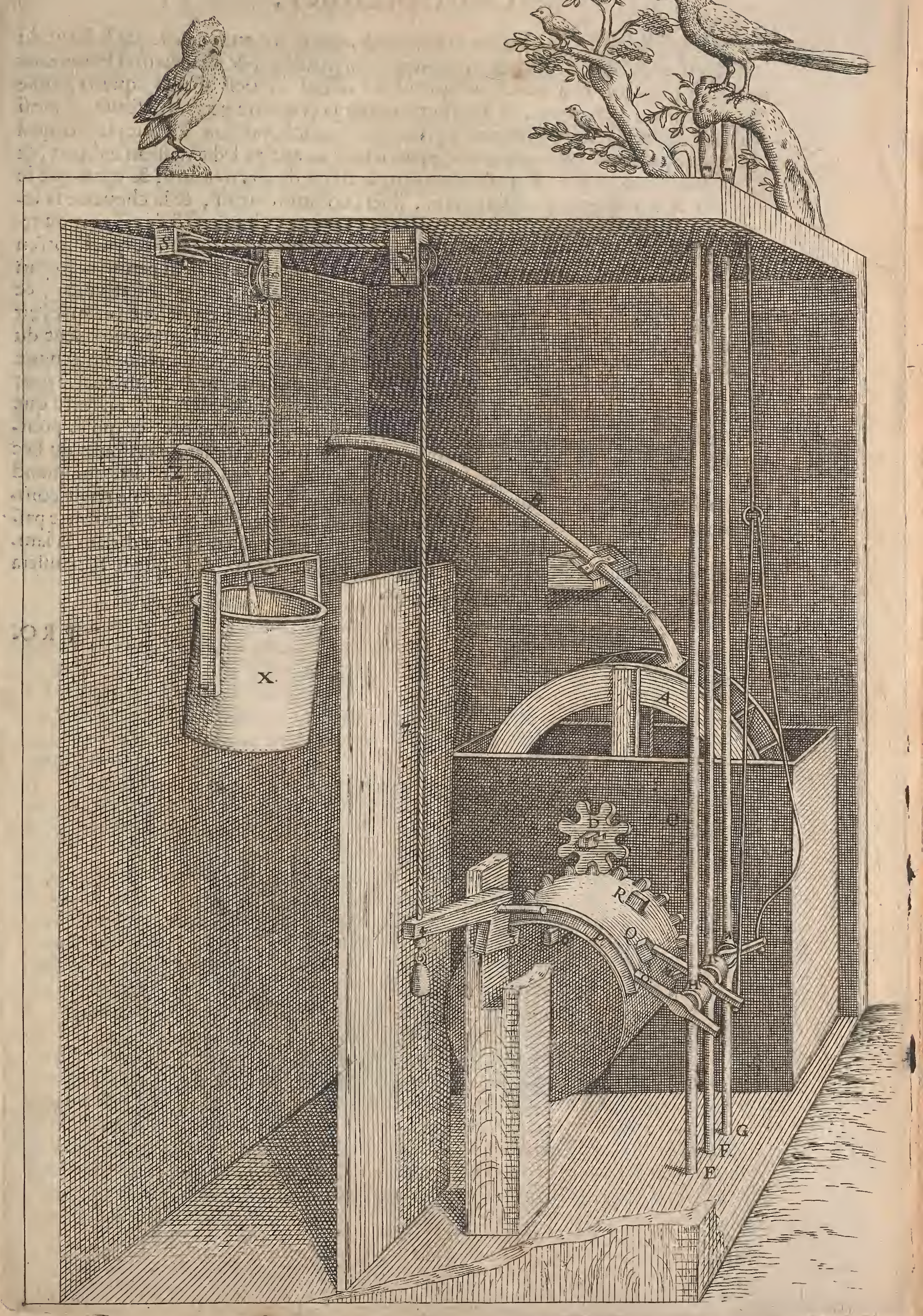




\section{Liure premier,}

( ̀̀ caufe qu'elle eftoit arreftee par le tabourin, qui eftoit auffi arrefté par le bout du leuier marqué 3 . contre la cheuille marquee 6.) tournera $\&$ fera chanter les oifeaux auec le coucou, \& faut noter que quand le vaiffeau X. s'eft abaiffé, que la poulie S. a tourné vn demy tour, \& aura fait tourner la chouette vers les oyfeaux, \& ainfi le chant defdits oyfeaux continuera, iufques à ce que le vaiffeau X. foit plain \& quill fe renuerfe, alors le contrepois du bout du leuier 4 . atirera ledit vaiffeau en haut, \& fera arrefter le tabourin, \& par confequent le chant defdits oyfeaux, \& apres que le vaiffeau X. fera derechef à demy plain, il fera comme deuant, \& la chouette fe retournera vers les oyfeaux, lefquels recommenceront à chanter, \& ainfi ce mouuement continuera iufques à ce que le vaiffeau de bas foit plain d'eau, \&rque l'air n'en forte plus, \& pour faire vuider l'eau dudit vaiffeau, l'on fera vn petit pertuis au tuyau en bas dudit vaiffeau, d'où l'eau fortira toufiours iufques à ce qu'il foit vuide, \&c faut garder que ledit trou ou tuyau ne foit trop grand, car il empefcheroit que l'air ne fortiroit comme il faut pour le chant des oyfeaux, \& quand au mouuement du coucou il fe fera en cefte façon, Soit la figure marquee $A$, faite de plomb ou cuiure creufe par dedans en forteque l'on y puiffe adapter vn mouuement, comme il fe peut aifement comprendre par la figure, la partic inferieure du bec fera faite en forte que la reigle de cuiure B. hauffant par dedans, puiffe faire ouurir le bec, ce qui fe pourra faire parle moyen d'vne petite cheuillete C. paffante au trauers le deffoubs dat bec \& à la queue de l'oyfeau, il y aura auffi vne reigle $D_{0}$ par dedans, en forte que qquand I'on baife le bout, que ladite queüe fe puiffe leuer, \& ainfi il y aura vne reigle com. mune marquée E. F. laquelle fera aufí fufpendue auec vne petite cheuillette paffante au pertuis $G_{0}$ \& alors quandl'on tirera vn petit filet (paffant par dedans la iam. be de l'oùfeau eftant attaché à ladite reigle) ) le bec s'ouurira, \& la queuë fe hauffera comme il fe peut comprendre par la figure. 


\title{
Des forces mouiuantes.
}

B土-

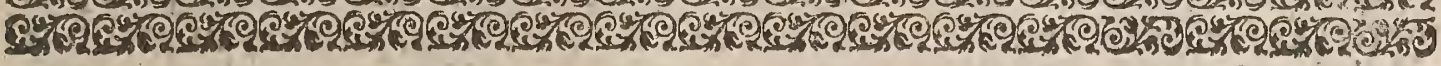

\author{
PROBLES M X X I III.
}

Machine par laguelle lion reprefentera one Galatee qui fera trainee fur leau par deun daufins, allani en ligne droite, ES (e retournant d'elle mefme, cependant qu'vn ciclope ioüe deffus wn flaiolet.

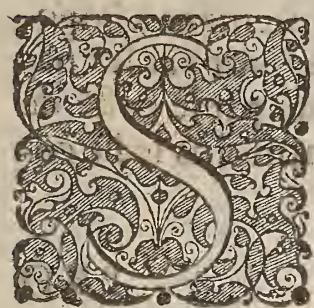

Orr vne rouè à eau marquee $\mathrm{L}$. dont la largeur fera feparee parle milieu, \& que d'vn cofté les augets ou tombent l'eau foient faits pour tourner de la main dextre, \&à l'autre cofté ilsferont faits pour tourner à feneftre, \& à laxe de ladite rouë, il y aura vn pignon qui fera tourner vne rouë dentelee marquee $Z$ \& \& ladite roue aura vn axe auifi diuifé en deux, marqué 1 . H. \& à ladite axe, il y aura deux chaines paffees, en forte que quand ladite axe tourne, que I'vne defdites chaines puiffe tourner à l'entour, \& lautre fe deftourner, lefdites chaines pafferont parles poulies G. F. \& feront toutes deux atachees à la poulie B. mais

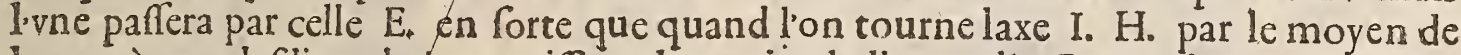
Ja rouè cau, lefdices chaines puiffent, l'vne cirer ladicepoulie $B$. vers le mouuement, mais fi l'on fair tourner la roue à eau de l'autre cofté, alors la chaine qui paffe à trauers la poulie E. atirera celle B. à foy, \& l'autre chaine fe delachera à proportion,

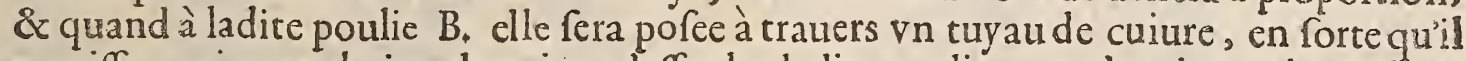
y puiffe auoir vne platine de cuiure deffoubs ladite poulie entre les deux pierres longues C. D. de façon que ladite poulie puiffe gliffer facillement fur lefdites pierres, \& que le tuyau A: fe puifle toufiours tenir droit fans varier d’vn colté ny daurre, mais tourner quand ladite poulie $B$. tourne, \& fur ledit tuyau $A$. l'on airftera vn autre tuyau $N$. en forte que celuy A. puife entrer bien iuftement dedans, \& ledit tuyau pourra conduire leau à la bouche \& narines des daufins qui trainent la coquille ou eft affife là Galatee, apres l'on aura vne petice caffe de plomb ou cuiure marquee $P$. de viron vn pied \& demy de long \& 2 vn de large, auquel il y aura vre Coupape foudee au fond, marquee $R$. \& au bout de bas dicelle vn tuyau marqué N. \& au milieu deladite caffe au cofté Q. il y aura vn tuyau marqué $O$. \& entre lefpace dudit tuyau, \& le fond de ladite caffe, il y aura vn petic tuyau, lequel donnera leau dans vn baffin marqué $S$, lequel fera ataché à deux trebuchets marquez $V$. T. en forte que quand le vaiffeau fera plain d'eau, qu'il puiffe eftre plus pefant que le couuerteur de la foupape $R$. \& au contraire, quand ledit vaiffeaufera vuide, il faut que ledit couverteur de foupape foit plus pefant, à celle fin qu'elle puiffe fermer \& attirer ledit vaiffeau en haut, \& ainfi quand leau donnera dans ladi. te caffe par le tuyau $V_{0}$ elle fe hauffera iufques au tuyau $O$, \& tombera fur le cofté de la roue à eau $L$. alors ladite roue tournante, fera tourner celle $I_{\text {. }}$ \& par confequent laxe I. H. de telle façon que la chaine fe tournera à lentour de I. \& re deftournera de $H$. alors la figure de la Galatee, fe mounera vers la pou. lie E. à caufe qu'elle y eft atiree par ladice chaine qui tourne à laxe I. \& faudra

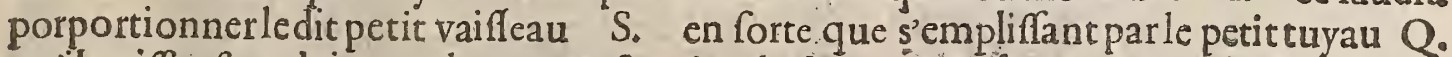
quil puiffe eftre plain au plus pres, \& atirer la foupape en haut quand la figure de $l_{2}$ Galatee fera proche de la poulie E. \& alors l'eau qui fera dans ladite caffe to mbera par la foupape fur le cofté M. de la roue à eau, \& fera tourner ladite roue delau. tre colté, en forte qu'il faudra que la chaine $H$. fe tourne à l'entour du cofté de. laxe $H_{+}$\& fe deftourne de $I_{\text {. }}$ ce qui fera caufe de faire retourner la figure vers le mouuement, \& alors l'eau ne courra pas dans le petic vaiffeau $S$. à caufe que la 


\section{Liure premier,}

foupape eftant plus baffe que ledit tuyau Q. empefche que leau ny peut plus monter, \& faudra qu'au fond dudit vaiffeau $S$. il y aye vn petit tuyau par ou fe: vuide ladite foupape $R$. fe referrera, qui fera caufe de faire remonter l'eau iufques au tuyau $O$. \& par confequent à celuy $Q . ; \&$ remplit ledit vaiffeau, \& ainfi la figure fe retournera vers $\mathrm{E}$. comme au precedent, \& ce mounuement durera autant, comme l'eau tombera fur la rouë L. tantoft d'vn cofté, tàntoft de lautre, Et quand au ciclope, lequel doir ioüer du faiollet, quand ladite figure fe mouue, le mouuement en fera enfeigné au fuiuant problefime, c'ef à dire pour faire ietter l'eau, au daufin, qu'il faut auoir vn tuyau à l'opofite de celuy A. deffoubs les pierres C. D. en forte que quand celuy $A$, vient à fe rencontrer iuftemant à l'opofite, que l'eau qui fort dudit tuyau puiffe entrer dans celuy. A \& fortir par les narines \& bouches des daufins; faut noter que la caffe $P$. eft outuerte par le cofté de deuant à propos pour voir le mouuement de la foupape. neantmoins ledit co fté doit eftre efgal aux autres.

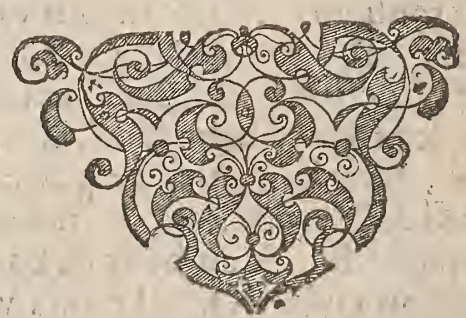



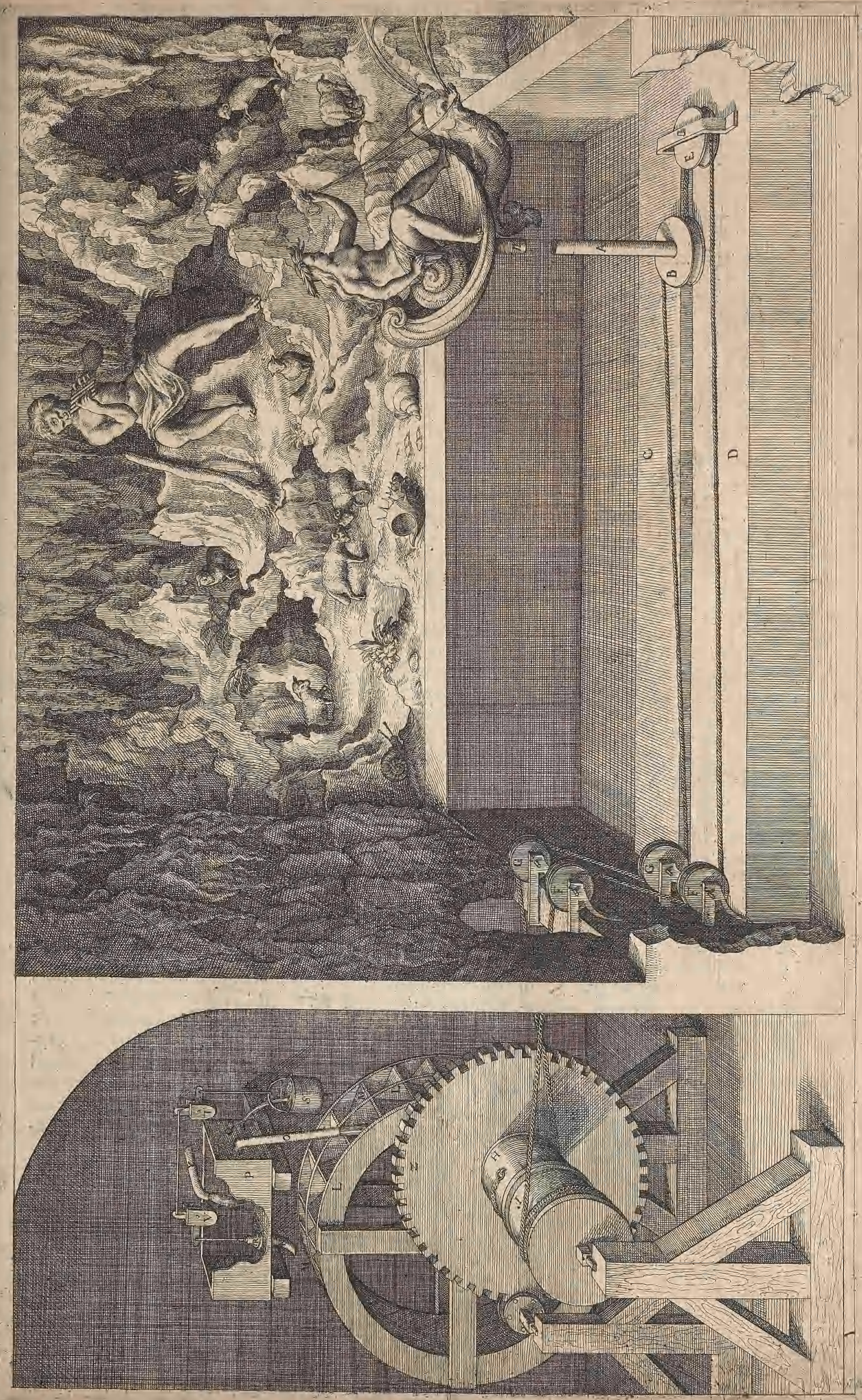


\section{Liure premier,}

1: Tojo

$P R O B L E S M E X X V$.

\section{Oratbine par laquelle bon reprefenters be fon divn flaiollet auec le cours de leaw.}

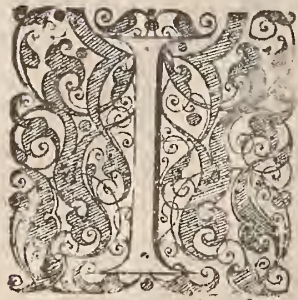

Cy fera reprefentee la machine propre pour faire fonnerle flaiolet au ciclope du precedent problefme, foit donques vne roüe muficale marquéce $A$. de viron 4 , Ou 5 . piedsen diamettrebien arondie tout à l'entour, \& gradue e de dents, comme il fe peut voir en la figure, en forte qu'vn pignon de 8. dents marqué B. puiffe faire tourner ladite roué, \& à larbre dudit pignon il y aura vne rouë d'entelee de 32. dents marquee $D$. qu'vn autre pignon marqué $C$. tournera, \& à l'arbre dudit pignon $C_{+}$il y aura vne rouè à eau marquee $F$. de viron deux pieds \& demy ou trois pieds en diametre, laquelle fera tournee par l'eau defcendance du tuyau G. \& ainfi quand ladite rouë tournera, elle fera tourner la rouë muficale par le moyen des autres rouës, apres l'on pofera le fommier marqué H. dont la fabrique fera monftree plus amplement au troifiefmeliure, en forte que les touches dudit formmier aprochent paralelles à vn demy pouce pres ladite rouẹ muficale, apres l'on diuifera ladıe rouë muficale en 25 . ou 30 . parties efgalles chacune partie en tournant, fera vne mefure ordinaire de mufique, \& en outre, toutes lefdites parties feront diuifees en 8. pour pofer ( $s y$ befoin eft) des crochets fur chacune diuifion, done en faut 8 . pour vne mefure, \& fi l'on veut, lon y pourra enco. res pofer des demis crochets, apres pofer les cheuilles fur ladite roüe, fçauoir $\frac{3}{4}$ de pouce en dehors, la fuperficie de ladice rouë muficale, en forte que quand la roue cournera, lefdites cheuilles puiftent toucher les toucnes du fommier, \& les abaiffer pour faire ouurir les foupapes dudic fo mmier, quand aufdites cheuilles elles fe poferont felon la chanfon qu'on defre faire fon nerau flaiollet, celle qui efticy pofee, commenceainfi

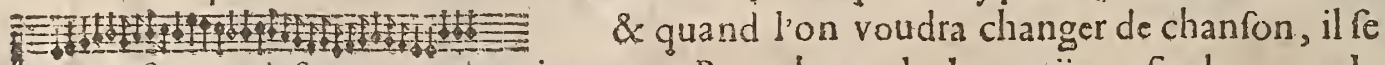
jourra farre, defmontant le pignon. B. hors de la rouë muficale 2 par le moyen d'vn apuy de fer marque L. fur quoy ledit pignon fera pofé ". \& de. lachant la perice vis marquee $N$. qui tient ledir apuy eneftat, alors ledit apuy fe rirera dehors fon trou, \& ledit pignon fera defioint de la roüe muficale, laquelle fe pouuant tourner auec la main, lon affoirra telle autre chanfon que lon voudra deffus ladite roüe, less douze trous qui font au fommier fer. uent pour porter le vent dudit fommier par des porteuents de cuiure ou de plomb aux pipes d'orgues pour reprefenter le fon du flaiollet, lefqulles feront tout ioignant la figure du ciclope, la conftruction defdits tuyaux fera enfeignee au troifiefme liure, $\&$ quand aux crochers qui pendent aux cordes $\mathrm{P}$. O . ils feruiront pour hauffer la roüe muficale en haut, à celle fin que s'il aduenoic quelque faute aux foupapes de dedans le fommier l'on y puiffe remedier, ouurant ledit fommier par deuant, com. me l'on fait ordinairement, le grand porteuent marqué R. S. pourra eftre de bois de quatre pouces en quarré pour conduire le vent au fommier, lequel viendra des fouflets, comme fera enfeigné au troifiefme liure, mais $s^{\prime} y$ l'on vouloit faire iouër ledic flaiollet fans aucuns foufters, alors il faudroir faire comme fera enfeigné au problerme 3ः。 


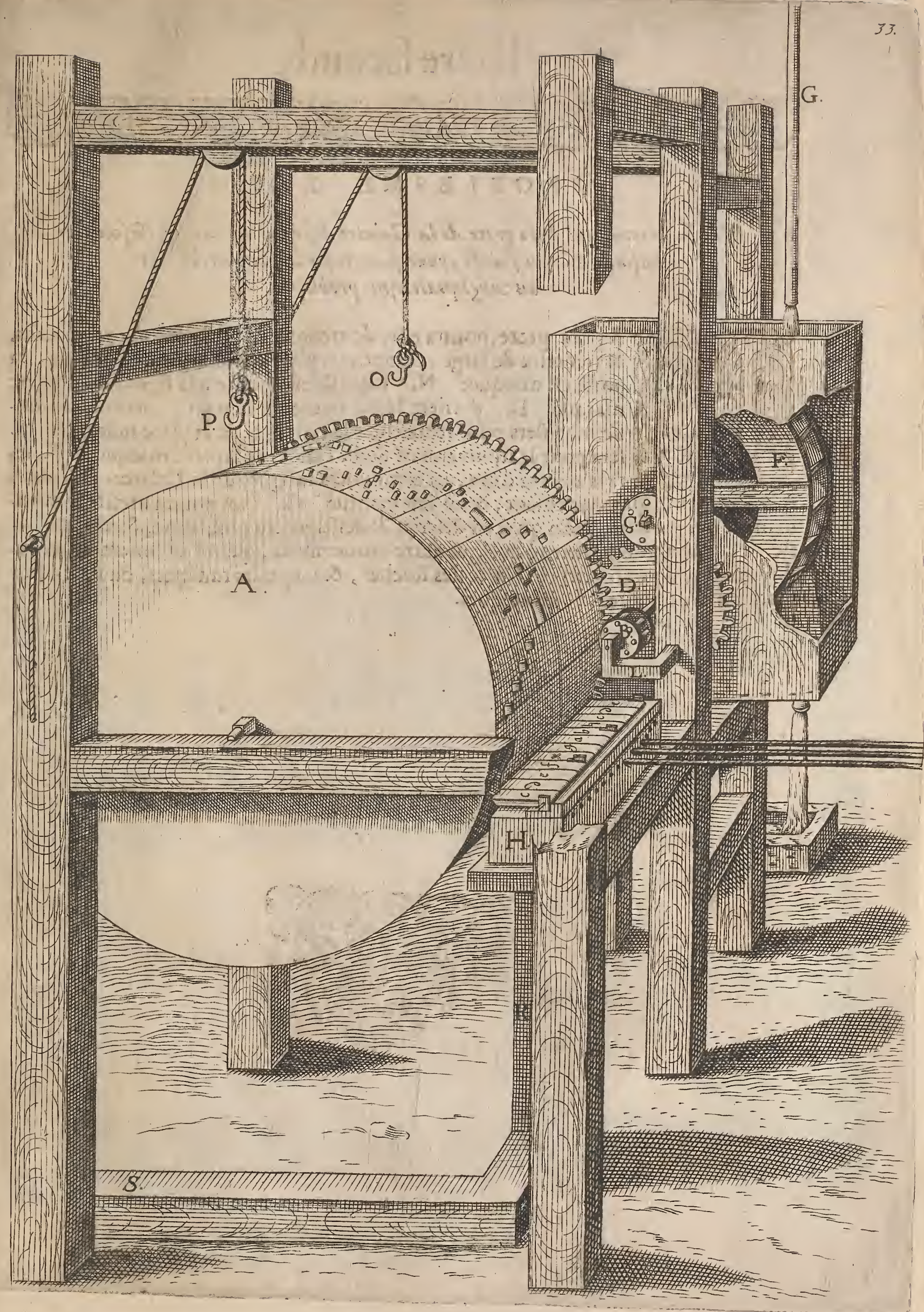




\section{Liưrefecond,}

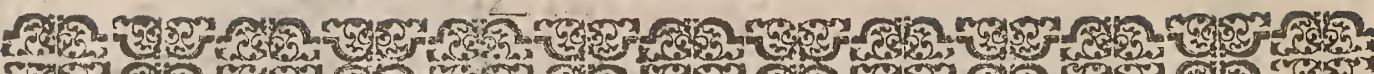

B.

\section{P R O B L E S M E X X V I.}

Plan Fngmografique de la grate de la Galatee deferipte au vingtroisiefme prow ble fme, E' celus a außi, pour faire joüer le Flaiollet defcript au vingtquatrie/me proble $/$ me.

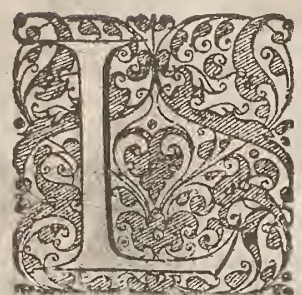

A DITE grate, pourra eftre de trenteneuf pieds de long par dedans, \& trentefix de large, compris les places pour les mouuements, la porte eft marquee $N$. laquelle eft opofite à la figure du ciclope marqué D. derriere ladite figure au lieu C. feront les doufiefme fiflets pour reprefenter le Flaiollet, \& la place marquee B. fera pour le mouuement dudit Flaiollet, la place marquee F. fea referve deau, ou fe mounera la figure de la Galatee, \& la place A. fera pour ton mouuement, \& au lieu marqué G. l'on pourra mettre le mou. uement des fouflets, felon qu'il eft defcript \& deffeigné au problefme, \& àl'autre cofté $H$. l'on pourra mettre quelque autre mouuement, quand à lornement de la. dite grotte, il pourra eftre fait auec des Roches, \& coquilles ruftiques, ouauec com. partiments de figures \& \& grotefques. 


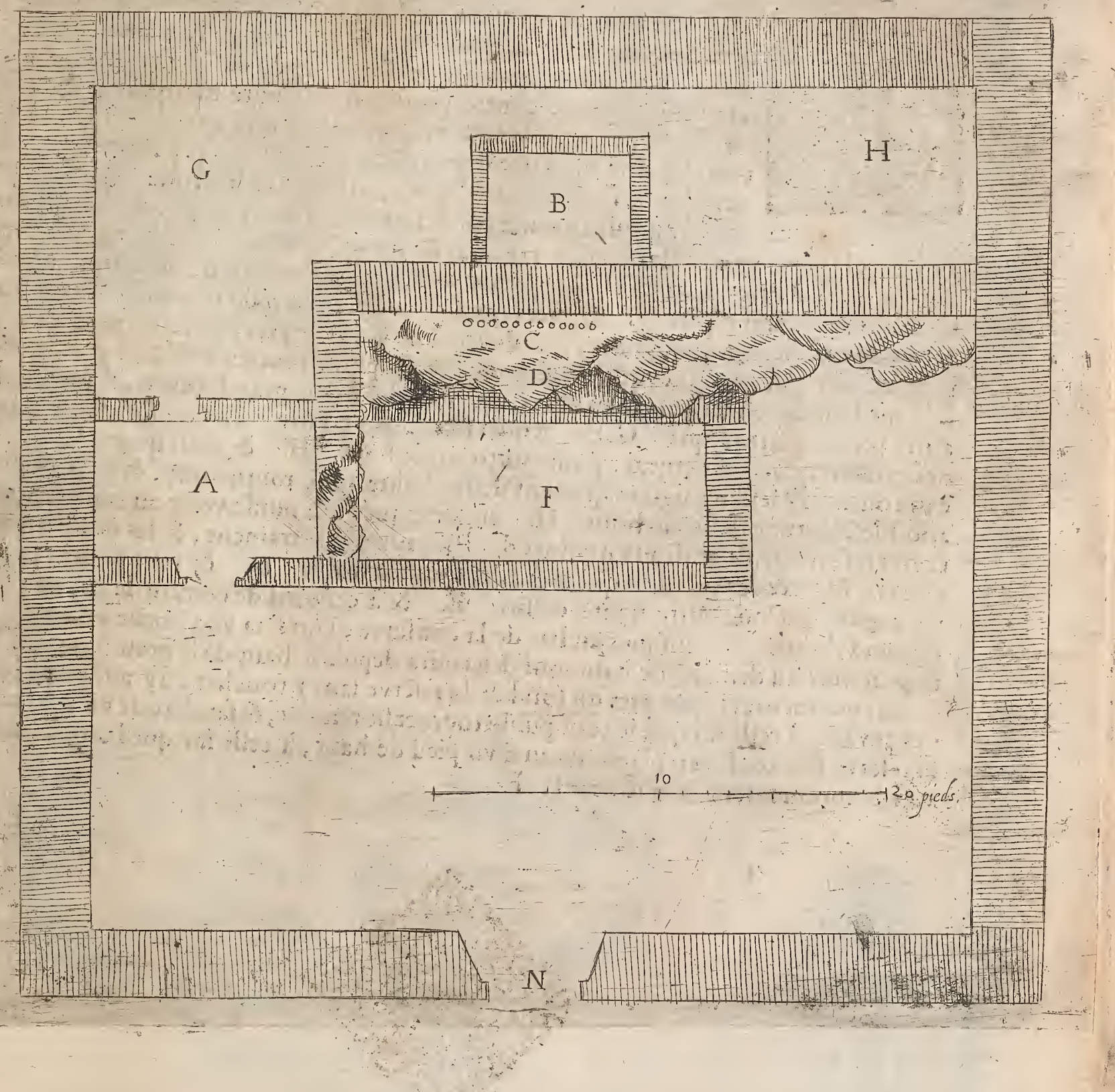




\title{
Liure fecond,
}

6) n.

\author{
$P R O B L E S M E X X V I I$.
}

SGachine, par laquelle fora reprefontê un Neptuno, lequel tourner a crculairement, àl'entour d'vne R oche, auec quelques autres figures, le gquel. lestetteront deleanen tournent.

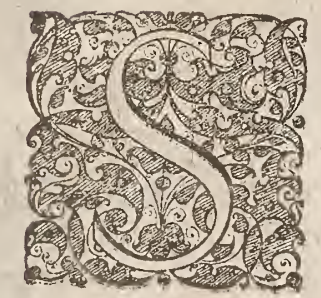

\section{Ort vne roüc àcau, marquee}

A. laquelle en tournant fera tour. ner vne rouë dencelé marquee

B. Ie pivor de laquelle fera apuyé deffus v ne piecde de bois droite, \& l'arbre de deffus marqué $P$. Q. fera foudé ferme, contrę vn tuyau de cuiure marqué S.R. \& au bour diceluy, il y aura vn peric recipien, ou tombera l'eau, apres il y aura vin autre grand tuyau, marqué $T$. V: lequel fera aulf foudé ferme contre larbre, vn peuplus bas, que $R$ en forte que ledit cuyas grand, puiffe tourner par deffus vn aurre tuyau, marqué de lignes punctees lequel fera entrelaxé, (marqué au(11 de lignes punctees, ) \& ledir grand ruy aux, \& celuy dentre deux fera foudé ferme, au fond de la referve de plomb marquee C. D. \& le grand tuyau T.V. fera foudé à vne grande rouë marquee E.F. la. quelle approchera à deux pouces pres du fond de ladite referve, en forte que quand la rouë de bas $B$. tourne, que ladice rouè E. Fo puiffe tourner aufi, d'autant quelles fone fermes, en vn commun accés, apres au deffus du grand tuyau, il y aura vn aurre petit tuyaumarqué G. H. lequel fera foudé contre $\mathrm{R}$. en forte que l'eau defcendante par lẹdit cuyau, puiffẹ fortir par le bour $H_{4}$ \& ainfi quand la rouë à cau toürnera, lefdites figures qui font deffus ladite rouę tourneront, \& l'on pourra affoir le Neptune deffus le bout $\mathrm{H}_{Q}$ en forte que l'eau puiffe venir au rrident, quil tient en fa main, \& auffi aux narrines des cheuaux qui le trainent, \& les deux tritons deffus $M$, \& le Cupidon qui meno les Daufins deffus $N$. \& l'on pourra enco: res mettre quelque autre figure deffus Z. \& à celle fin de couurir le tuyau; qui defcend depuis "S. iufques au bas de la conferve, l'on fera vne Roçhe, camme il fe peut voir au deffeing de haut, qui defcendra depuis le haut de la grote, ou fera ledie mouuement, iufques pres du fond de la referve fans y toucher, ny auffi à aucuns des tuyaux, à celle fin que le tout puiffe tourner librement, \& faudra que en tournant la referve foit toufiours plaine d'eau d'va pied de haut, à celle fin que l'on ne puiffe voir le mouuement de la rouë $E_{q} F_{\text {. }}$

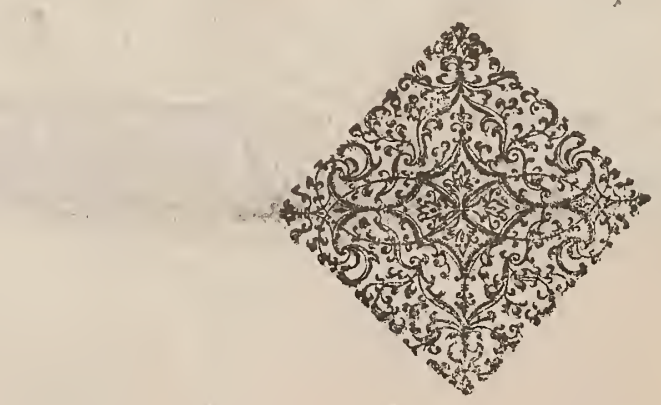



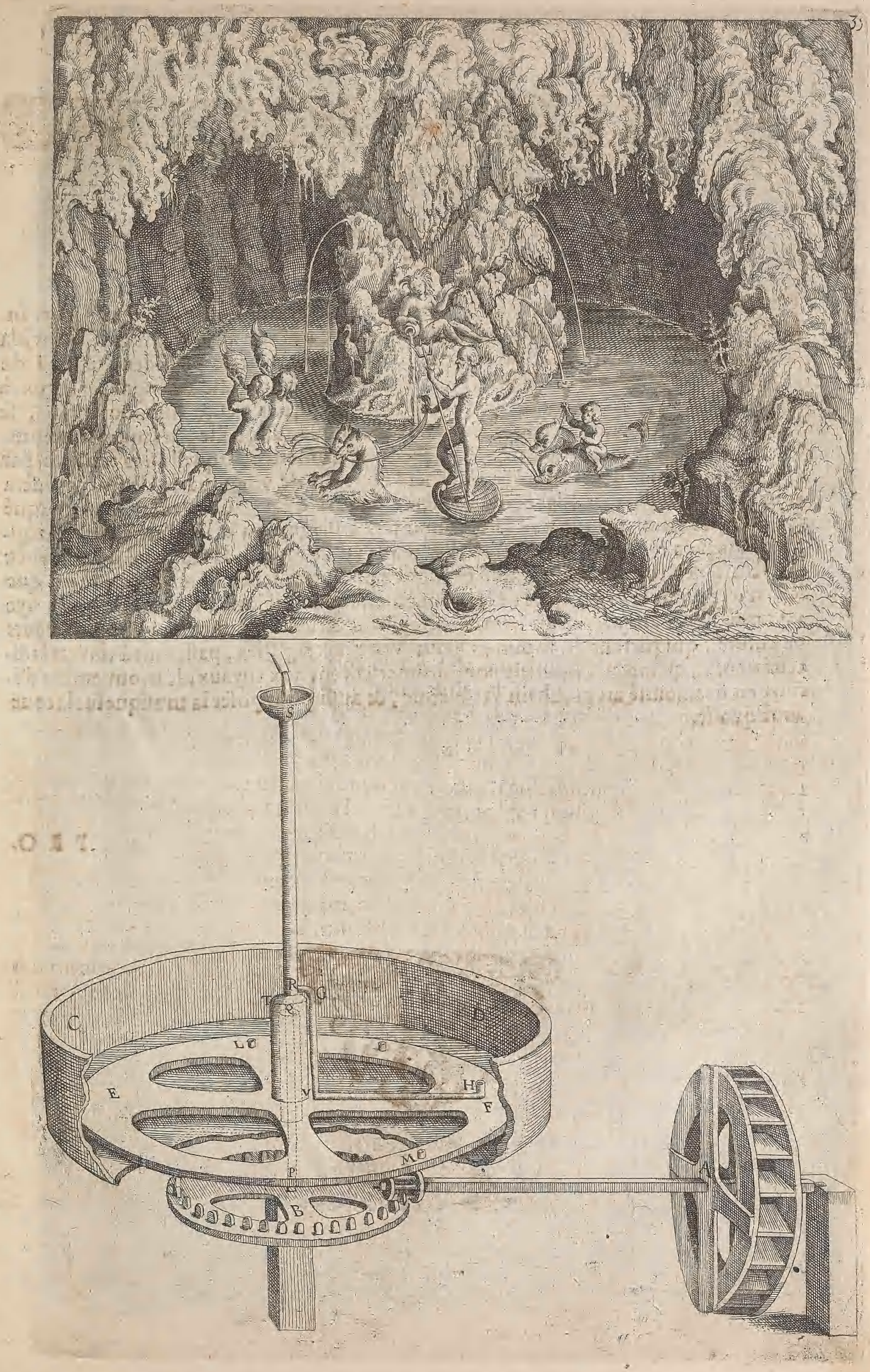


\title{
Liure fecond,
}

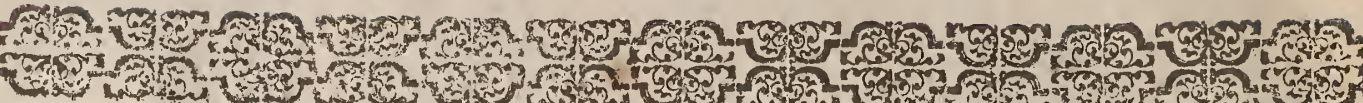

PROBLESME X XIII.

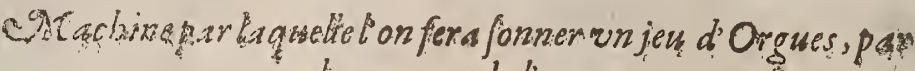 \\ te mayen delican}

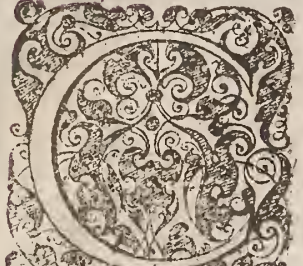

Esre Machine, eft fort femblale à celle demonftree au vingtcinquicince problefine, la difference del'vne à l'autre eft feulement à la diverte demontration des deffeins, car le precedent fe void de pourfile, \&reftuy cy de frond, \& cela a cfté deffeigné à propos, à celle fin que ce qui pouroir manquer deftre entendu à l'vn, fe . puife recoutrer a lautre, la rouë mulqqualle, marquee $A$. pour. vn pignon de huic ra efre de cing aे fix pieds en diamettre, laquelle fera tournee par va pignon de huit dents a laxe, duquel fera vne raue de vingtquatre dents, qui fera tounnee par vn pignon à laxe, duquel fera vne rouie à cau C. leclavier eft marqué D. \& le fommier $F_{3}$ dont la fabrique fera enfeignee au troifiefme liure, les regiAres marquez G.H.I. font trois differens l'vn de lautre, la fabrique d'iceux auec la mafuke des tuyaux, feront aufienfeignez audrt troifiefme liure, \& z̀ celle fin que bon n'oye pourt le bruit, que fait le mouruement quand il jouë, il fera bon quil y aye vne murailie d'vn pied efpais, entre les regiftres \& ledit mouuement, les portevents do cuiure, qui partent du fommięr pout venir aux regiftres, pafferont à travers ladite muraille, quand aux foufflets pour donner le vent aux tuyaux, le mouuement d'i. ceux en fera donnća a prochlain Problefme \& auffi pour pofer la mufique furląrouë mufiqualle
\end{abstract}

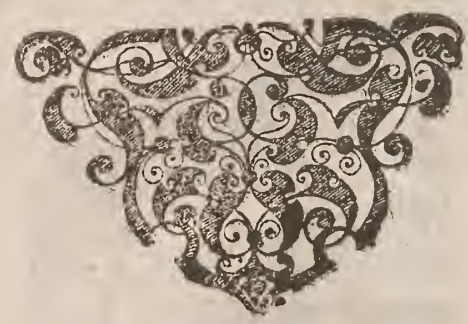




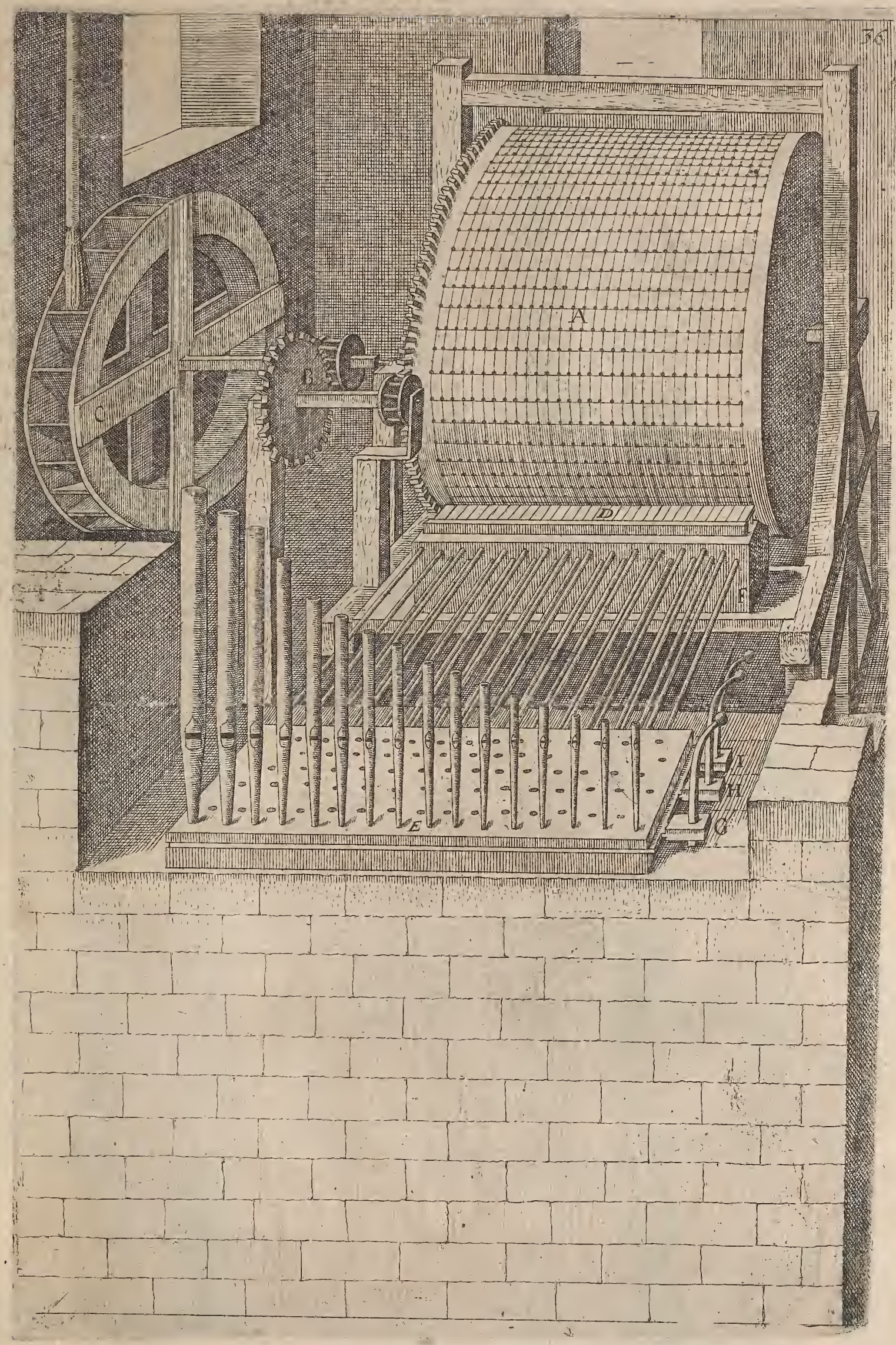




\section{Liure fecond,}

73.ro 4.

\section{PROBLES ME XX IX:}

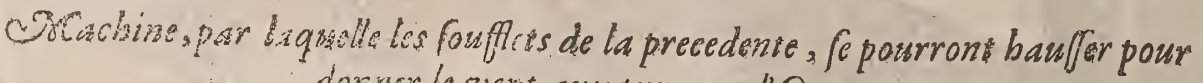
downer le vent aux luyaux d'Orgues.
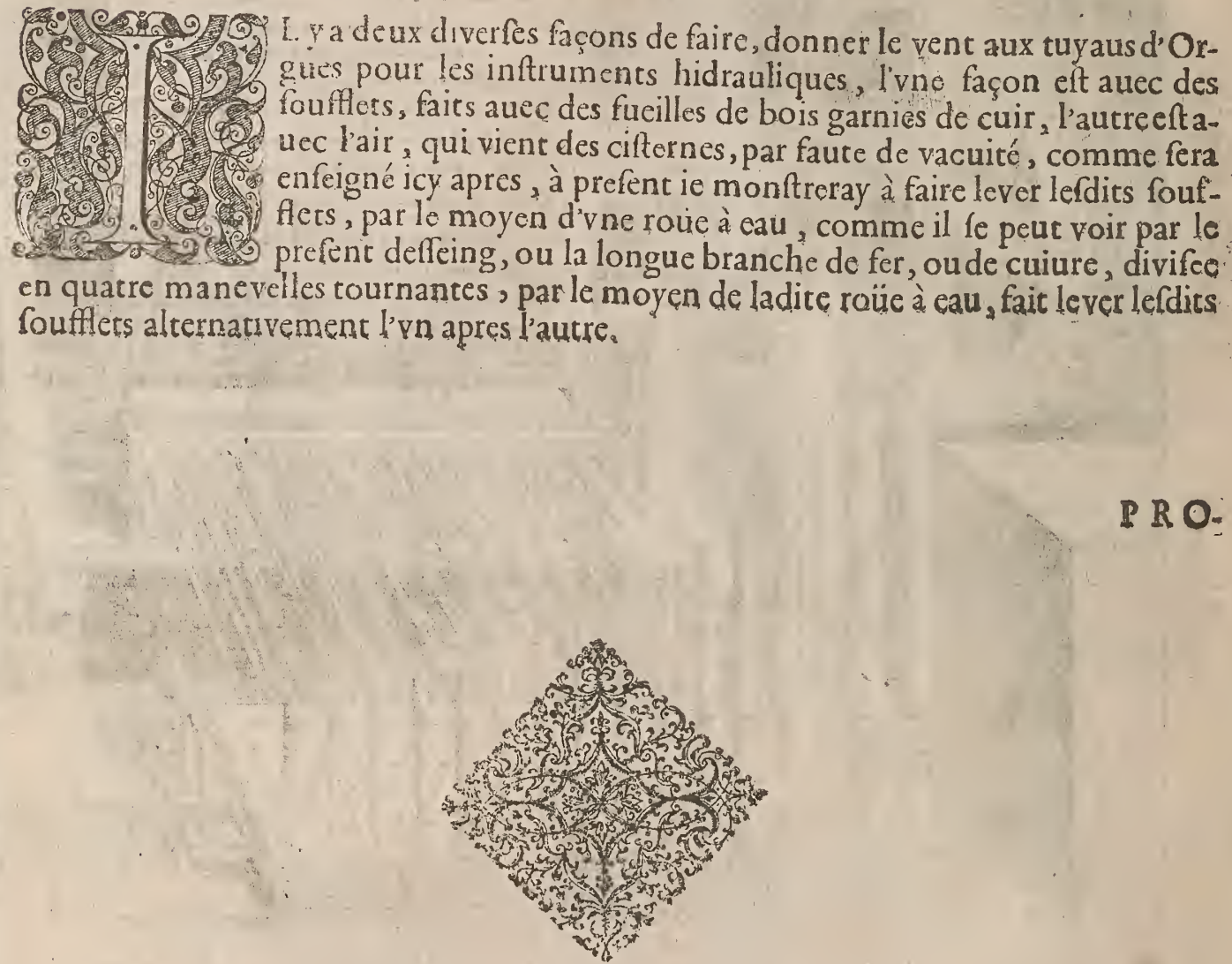


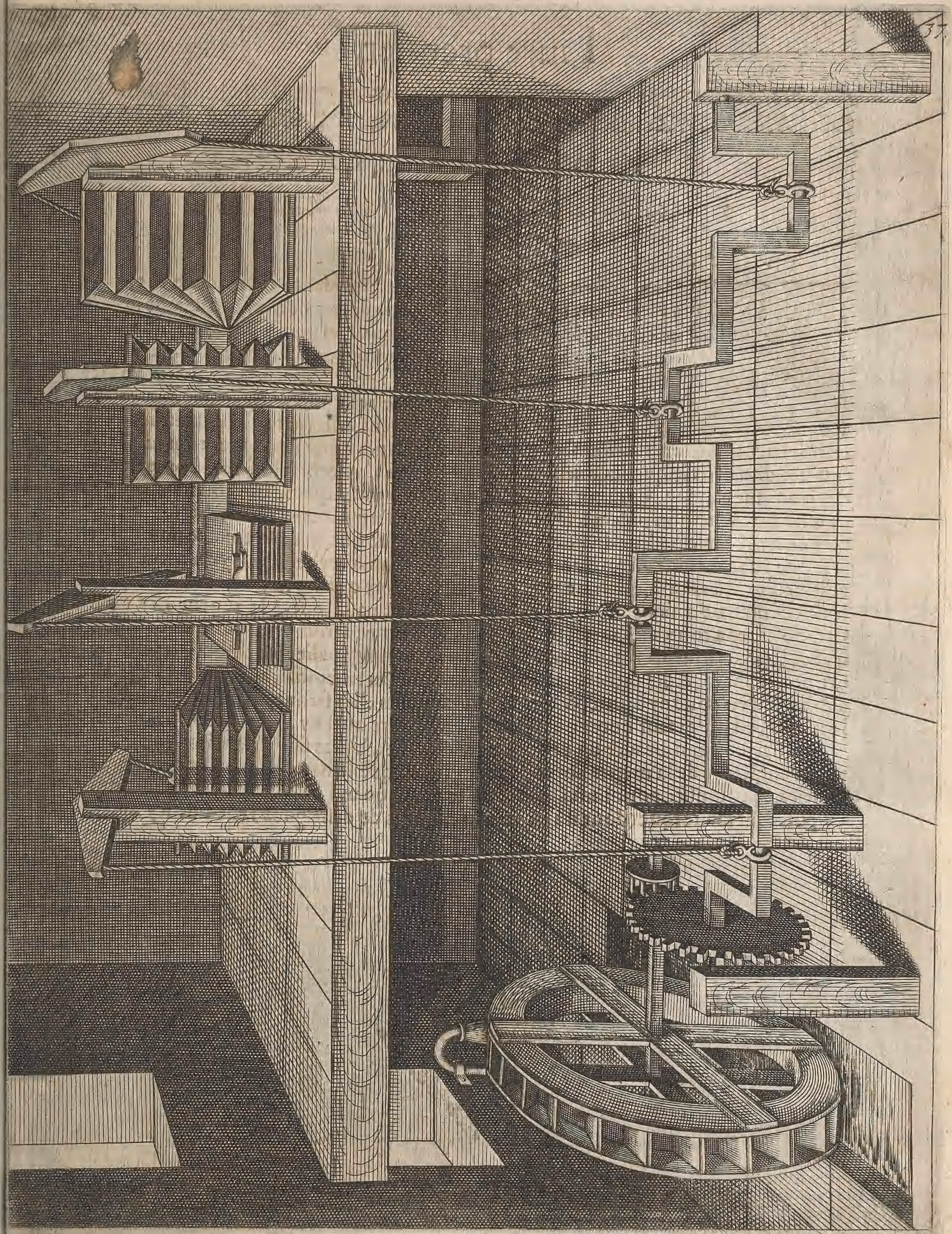




\section{Liure premier,}

ว

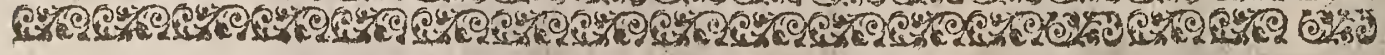

\section{PROBLESME XXX.}

\section{Reprefentation de la Roüremufiqualle, en plus grande formse pour fersir au proble/me 28 .}

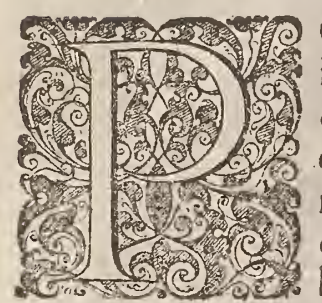

OVR entierement demonftrer la precedente machine ie mettray icy vne reprefentation d'vne partie de la roüe mufiqualle auffigran. de comme le naturel à celle fin que l'on puif́e voir parfaitemene comme les cheuilles abaiffent les touches du clauier, ladite partie reprefente feulement fis mefuress, dont lune fera marquee de noir ou de gris, tour du long de ladite roue \& l'autre fera marquee de bianc, a celle fin de plus facillement difcerner lefdites mefures, en curre chacune mefure fera diuifée en 8. parties, \& faudra tirer des lignes tout au long defdites diuifions lefquelles foient bien paralelles au clauier $\&$ fi lon veut l'on percera des trous fur chacune diuifion pour changer les cheuilles quand lon voudra changer de chanfon, apres lon pofera lefdites cheuilles, en fortequelles touchent fur le clauier enuiron de lef́peffeur d'vne defdires cheuilles, \& que lune ne touche point plus forr que l'autre, coutefois quand on viendra aux demis crochets dont y cra a feife pour yne mefure, ilfera bon qu'ils ne touchent point fi fort que les autres, a celle fin que lune cheuille ne touche auparauant que l'autre aye paffé outre la touche, ce qu'il faut obferuer a toutes les autres mefures, autrement ce feroit vne mufique confufe, quand a la fabrique de la roüe mufiqualle il eft befoing quelle foit de bois de chefne extremement fec \& les pieces bien afemblées \& colées enfemble, a celle fin quelle ne s'ente ny d'vn cofté ny dautre, \& quand aux cheuilles elles feront de cuiure ou de bois bien dur, en outre faut noter quen la prefente figure quill ny a que la moitié du cla: uier deffeigné, auffi beaucoup de feintes manquent a ladite figure, a raifon quele papier a empefché de la mettre entiere, aufí grande que le narurel, mais ce quill y ade deffeigné peut fufire pour lineelligence du refte, \& quand a la piece de mufique qui eft pof'é fur ladirerouë (done il s'en voit fis mefures de deffeignez) elle fuir icy apres. 


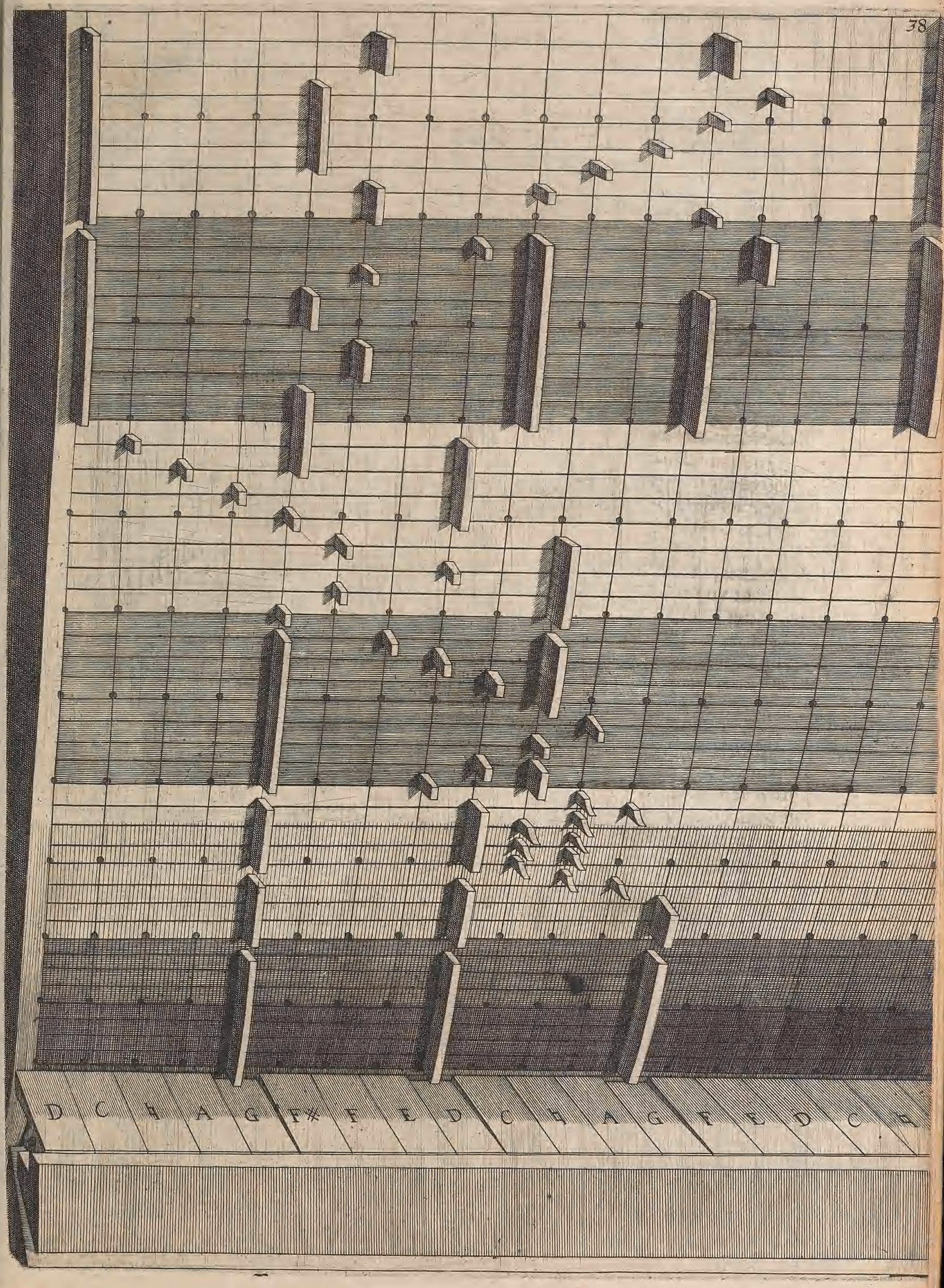




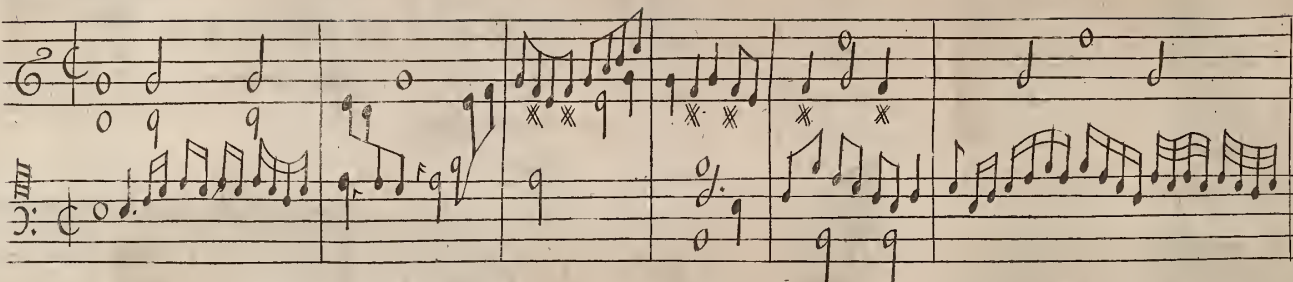

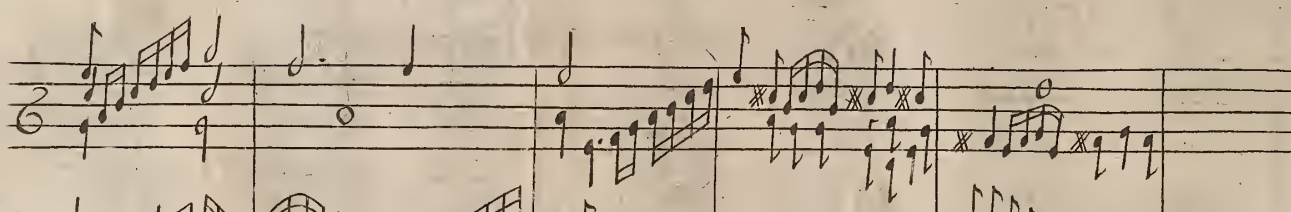

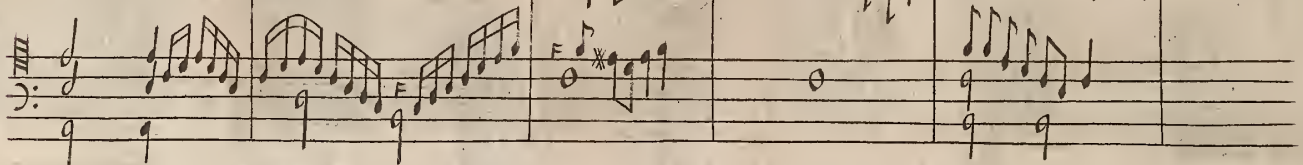

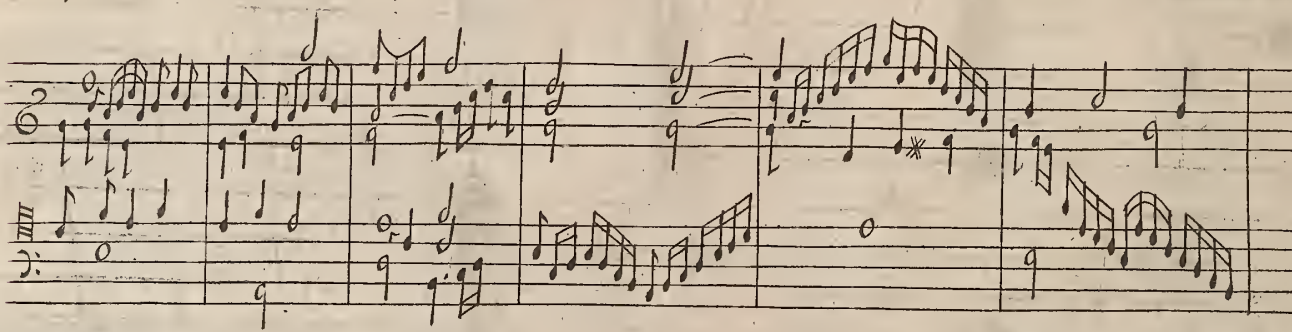

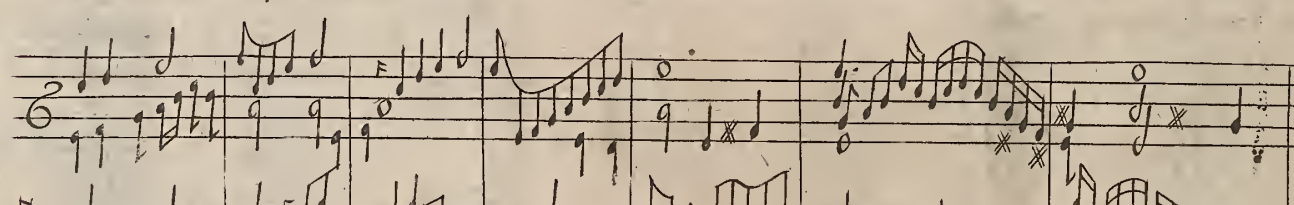

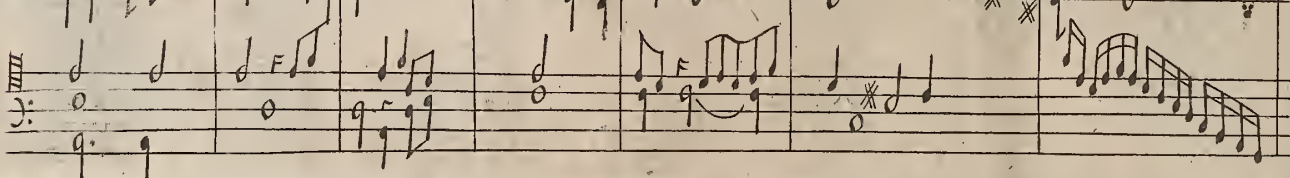

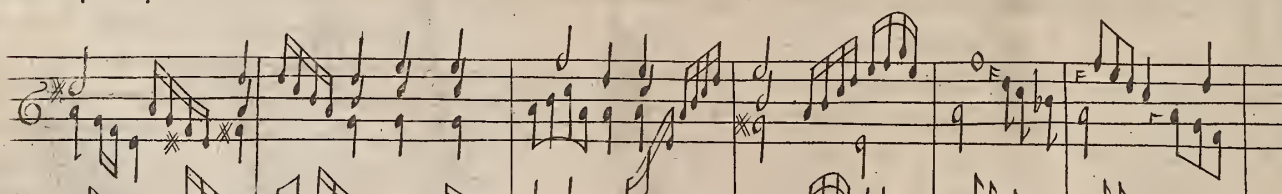

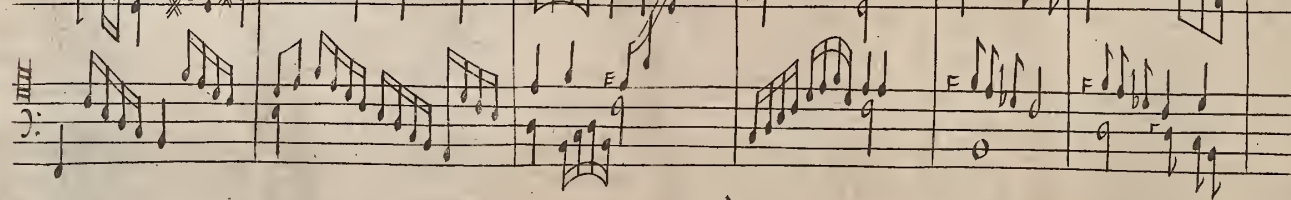

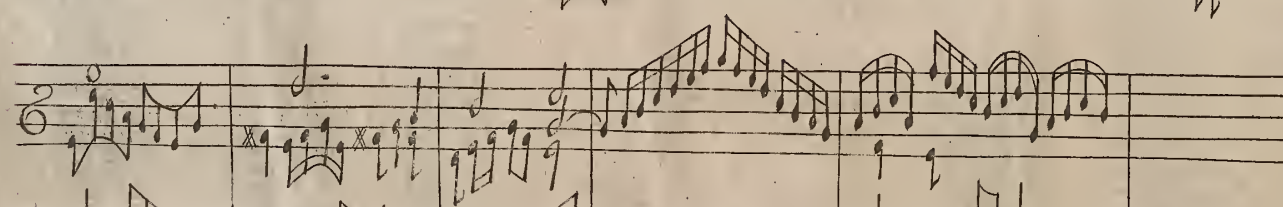

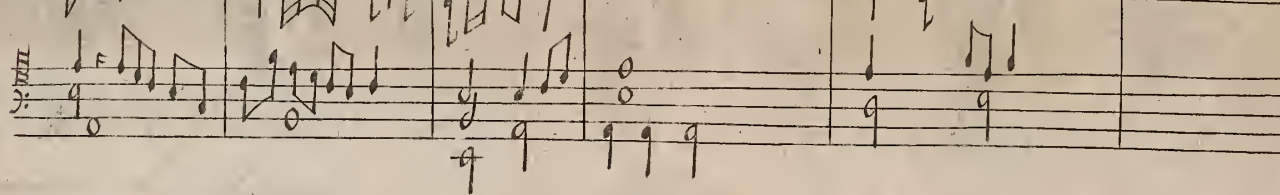




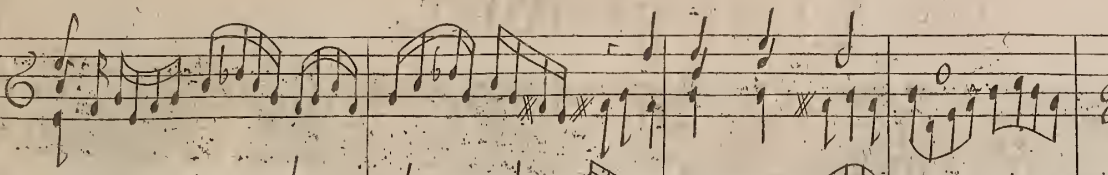

$\frac{1}{4} \frac{1}{4-14}$ I I I I I I I I

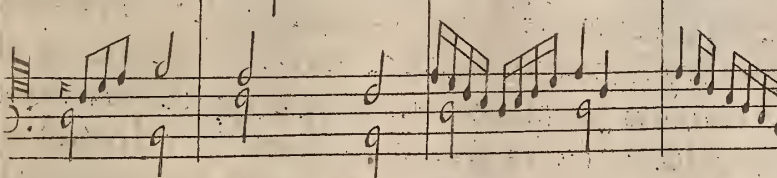
In I I I I 1 \&

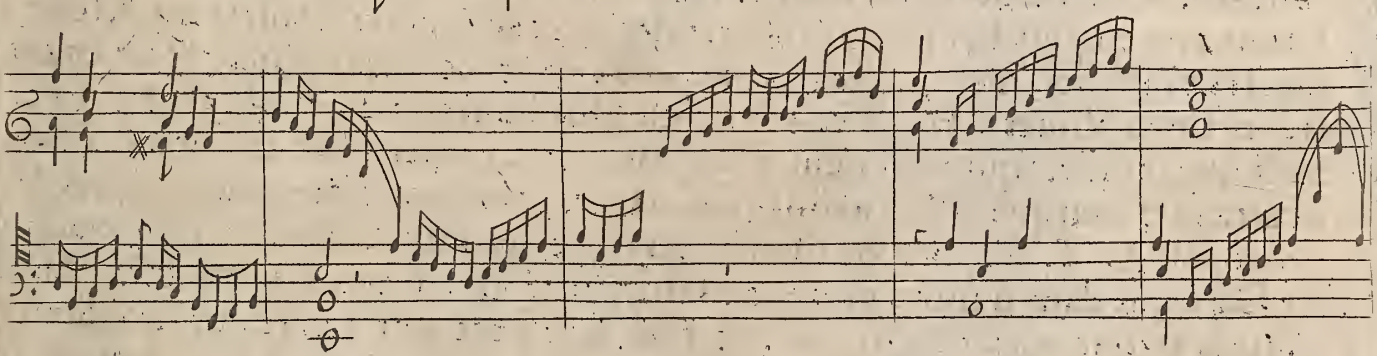
4 प

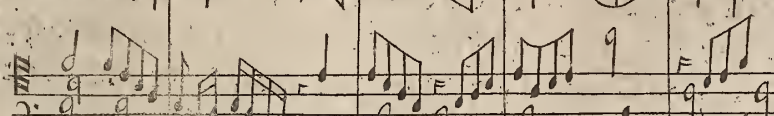

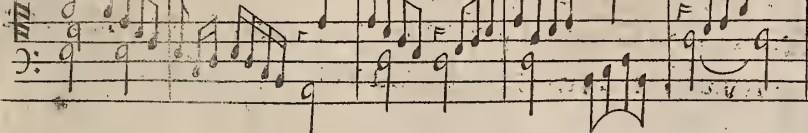

\section{$\frac{0}{111}=$}

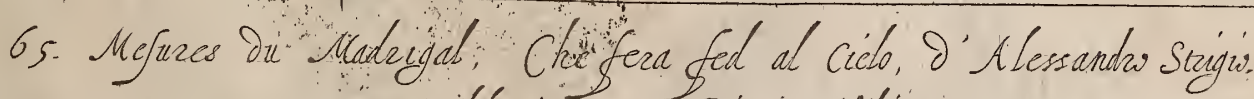
misen Tiblatire par Prence frifiper. 


\title{
Liure premier,
}

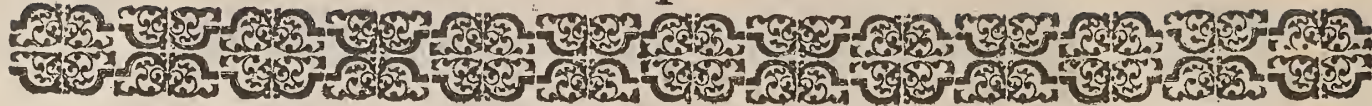

\author{
PROBLES ME XXXI. \\ OrCachine bidrauligue, par laguelle des orgues pourront fonner, \\ auecleaulans aide de fouffers.
}

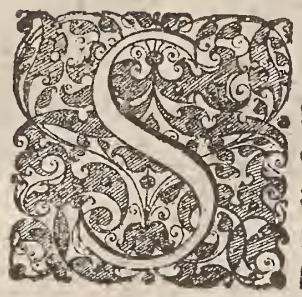

OIT vne roüe mufiqualle marquec

A. laquelle aura fon moruement, commelaprecedente, \& au lieu que le clauier eft deffus le fommier, icy il n'y aura aucun fommier, mais les touches du clauier comme ilfe vold aurone chacune vne longue queüe marquee $B$. au bout de bas, de laquelle fera ataché vn long filet, lequel riendra ferme a vne branche, au point C. \& ladite branche fera bien feigné parcideuane au problefme fixfiefme, \& ledir robinet fera coudé contre vn en porteuent marqué E. en forte que quand la touche B. fera abaifiée par les cheuilles de la roüe mufiqualle, la queuë de ladite touche atirera ladite branche C. \& fera ouurir le robinet D. \& le contrepois marqué F. fera referrer ledit robinet, auffitont que la touche fe rehauffera, \& y aura aurant de robinets, comme de touches, \& les branches ou feront atachées les filets du fecond robinet marqué G. feront vn peu plus hautes que du premier, à celle fin que les filets qui feront atachés audites branches, n'empefchene point ceux de ce fecond robinet, \& les branches du erentiefme robinet $H$. feront vn peu plus baffes que de celuy $D$. à celle fin aufis que les filets qui y doiuent eftre atachées n'empefchent les autres, \& celles du quartierme marqué $P$. feront plus hautes pour la mefme raifon, apres les porteuents I. L. T. V. feront foudées au bout des robinets par vn des bouts, \& l'auere fera ioint dans d'autres portcuents qui pafferont à trauers la muraille $M$. N. fur lefquels feront polées les tuyaux, comme il fe peut voir en la figure, \& fur chafcun porteuent, il y aura deux tuyaux a loctaue l'vn de l'autre, où a lunifon, \& fi l'on y en veut mettre d'auantege, on le pourra faire, ie n'ay mis icy que quatre robinets, pour efuiter confufion, mais comme ces quatre font faits, tous les autres feront femblables, le gros porteuent qui vient de la conferue à vent, fera foudé contre les deux ou font foudées les robinets, à celle fin que le vent foit bien communiqué audits robinets, \& delà aux tuyaux, \& a celle fin qu'il ne manque rien que ladite machine ne foit bien entendue ie feray encores les deffeings fuiuants.

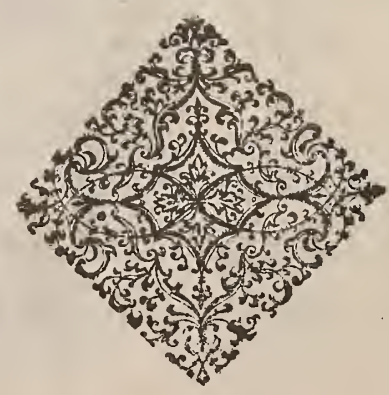




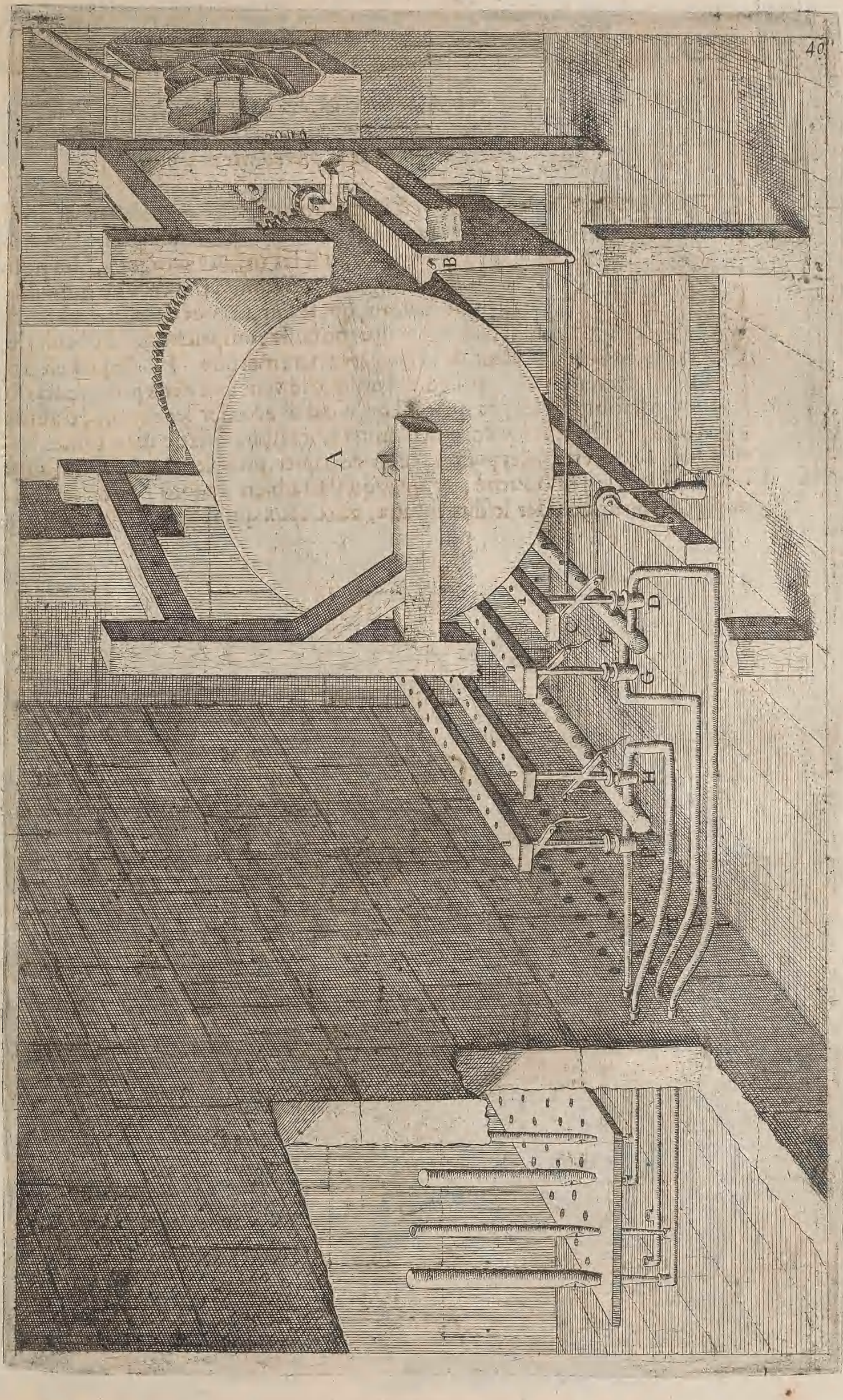




\section{Liure premier,}

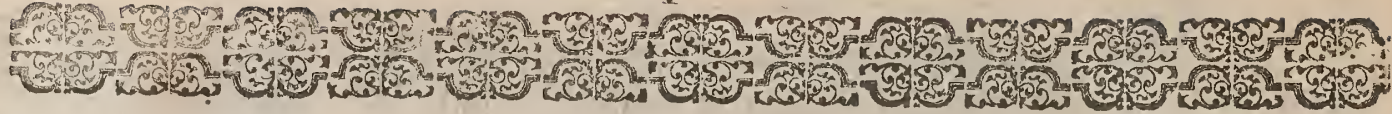

\section{PROB L E S M E X X X I I.}

\section{Autre deffeing, de la precedente machine.}

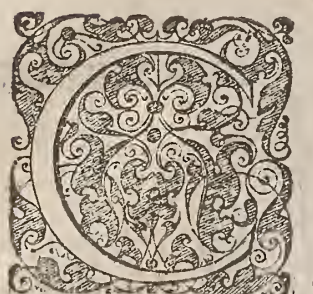

E deffeing icy eft la mefme machine precedente, mais il eft deffei. gné d'vn autre cofté, en forre que grande partie des robinets, fe peuuent voir icy, \& auffi les tuyaux d'orgues, les portevents fur lefquels font les tuyaux, pafferont à travers vne muraille, a cellefin que le bruit de l'eau, qui fe fait au pres de ladite machine, ne foit ouy fi fort, carladite muraille, emperchera ledit bruit, le portevent

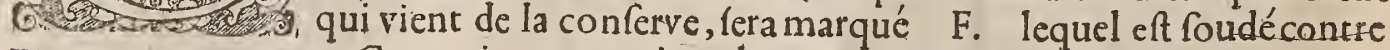
vn autre portivent Go qui communique le vent aux deux portevents, furlefquels les robinets font foudés, \& quand l'on voudra acorder les tuyaux, d'autantqu'il ny à point de regiftres, voicy comme l'on fera, il faudra mettre dans toutes les bouches, (des tuyaux ouverts, ) des petites pieces de papier, pour les engarder de fonner, apres l'on acordera le jeu bouché, \& apres qu'il fera bien d'acord lon ofteralefdites pieces de papier, pour acorder lefdits tuyaux, auec ceux qui font defia d'acord.

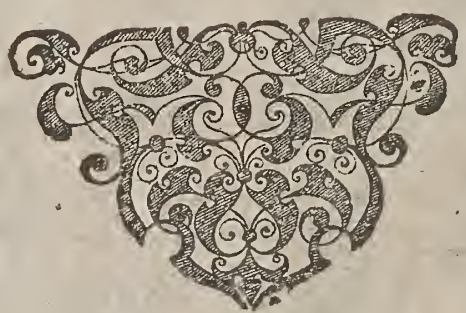




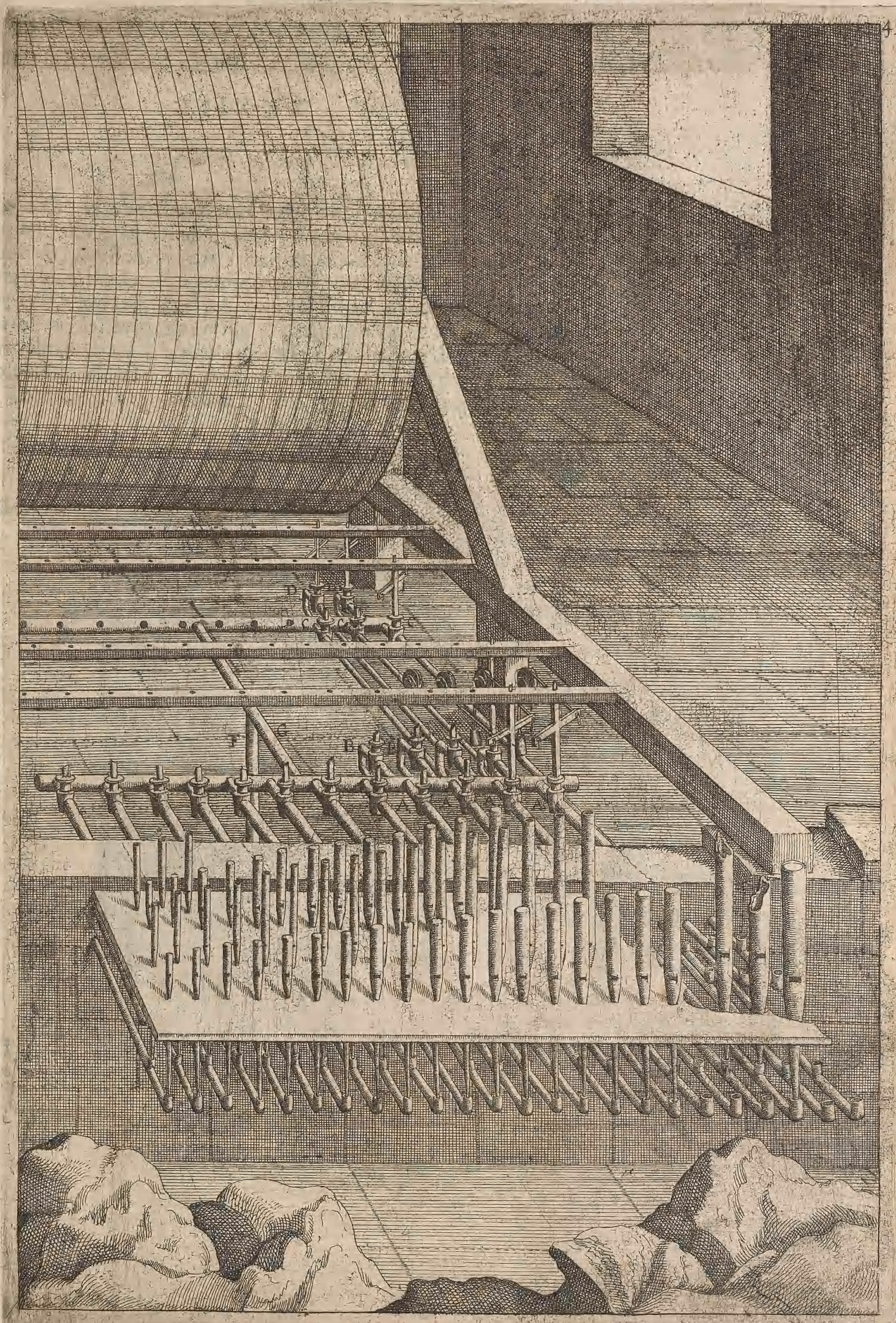




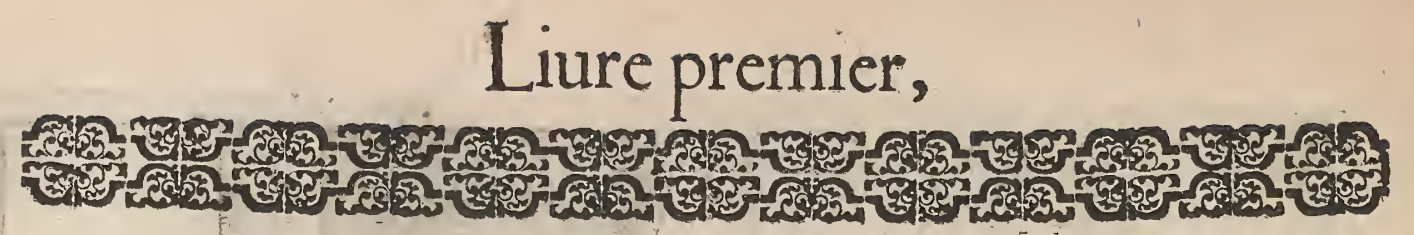

\section{PROBLESME XXXIII。}

Plan Ingnografique, de la precedente machine bidraulique.

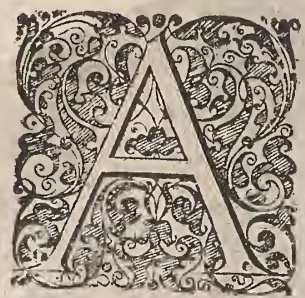

Crerr fin qu'il ne manque rien à lintelligence de la preceden. te machine, i'en demoniftreray icy le plan de lingnografie, les robinets deffeignés, aux precedentes parleslettres A. B. C. D. font icy arangées par ordre auec leurs nombres correfpondans, aux porteuents, qui paffent outre la muraille, les autres qui doibuene eftre foudées contre les robinets, \& aiuftées contre lefdits portefe que ledir plan uents, font obmis audit plan, à caufe que lẹurs obliquité, feroit cauqui n'eft deffeigné icy, fe peut facillement recognoiftre aux deffeings precedents.

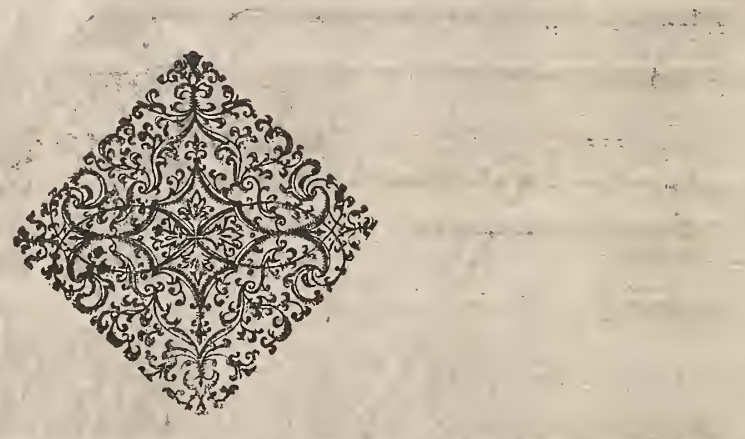




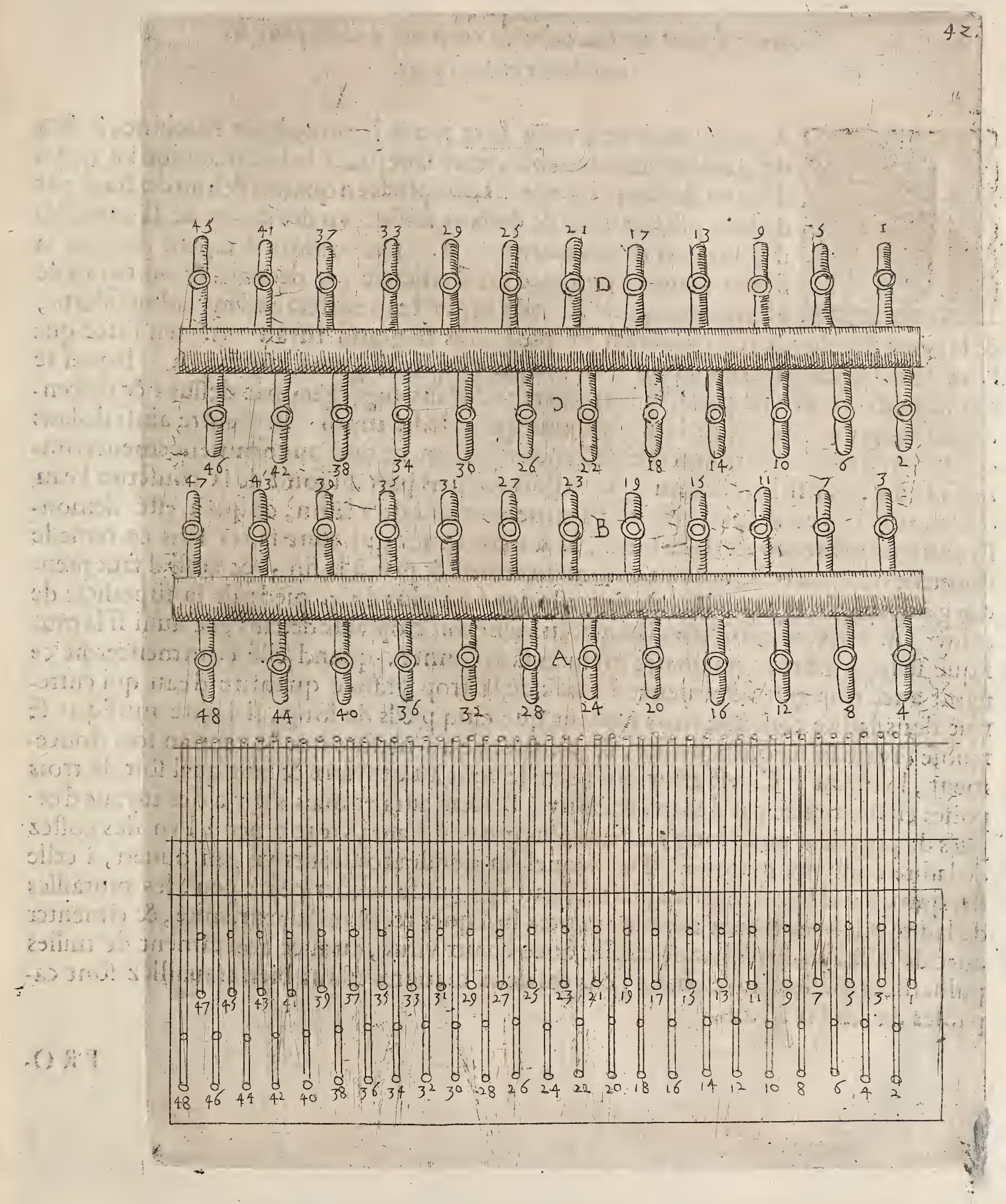




\title{
Liure fecond,
}

P R O L E S M E X X XIIII.

\section{Comme il faut conitruire's ta conferue à vent pour les machines hidraliques.}

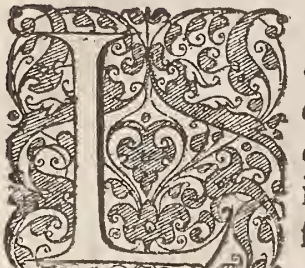

A conferue à vene pour faire jouër la precedente machine, fe ferá de grandeur conuenable, pour fairejouër ladite machine vn quare d'heure de fuitte, fi elle eft, dix pieds en quarré, \& huirde haut par dedans, fe fera affez \& dedans icelle à vn des coftez de la muraille il y aura vn tuyau marqué $A$. fait comme il fe peut voir par la figure denuiron vn pied en diamettre par dedans, \& vn tuyau de (a) plomb marqué B. par lequel l'eau entrera dedans laditecifterne, $\$$ le bout diceluy entrera enuiron vn pied dans le grand tuyau $A$. en forte que l'eau deffendante par ledit tuyau B. puiffe emplir le grand tuyau A. lequel fe maintiendra toufiours plain, \& l'eau encrant dedans regorgera par deffus, \& defcendra au long des coftez, \& là raifon pourquoy ledic tuyau $B$. entre ainfi dedans celuy A. eft à celle fin qu'elle defcende efgallement tant au commencementcomme à la fin, car fi ledic tuyau B. alloit iufques pres du fond de la conferue l'eau defcendroit beaucoupplus vifte au commencement qu'a la fin, ce qui à efté demonAtré au commencement de ce liure , \& les conferues qui font faites fans ce remede donnent beaucoup de vent au commencement \& peu à la fin, \& auffi il faut prendre garde que le tuyau $B$, ne foit eflongné au plus de 5 . pieds de la fuperficie de weluy A. car sil eftoit trop long l'eau viendroit trop viftededans, \& aufi fi la mu. fique fe repofoit trois ou quatre mefures fans fonner, quand elle commenceroit ce feroit auec trop grande violence à caufe de la trop grande quantité deau qui entreroit dans ladite conferue mais n'eftant que cinq pieds de long, fi ladite mufique fe repofe, l'eau fe repofera deffus, \& n'entrera qua mefure que le vent en fort doucement, le tuyau C. eft celuy qui porte le vent aux tuyaux \& faut quil foit de trois pones en diamettre par dedans \& celuy B. fera autant mais sil y a des tuyaux d.orgues de plus de 3 . pieds long, il feras plus gros, il y aura vn robinet à l'vn des coftez de ladite conferue marqué $D$. lequel on tiendra roufiours vn peu ounert, à celle fin que quand la cifterne fera plaine, l'eau s'en puilfe vuider peu à peu, les murailles de ladite conferue feront faites de petites bricques recuittes à lextremité, \& cimenter auec de la tiraffe de Hollande meflee auec chaux vifue, ou auec bon ciment de tuilles puluerifees meflees auec chaux, car ces deux matieres eftans bien trauaillez font capábles de refíter à l'eau.
\end{abstract}




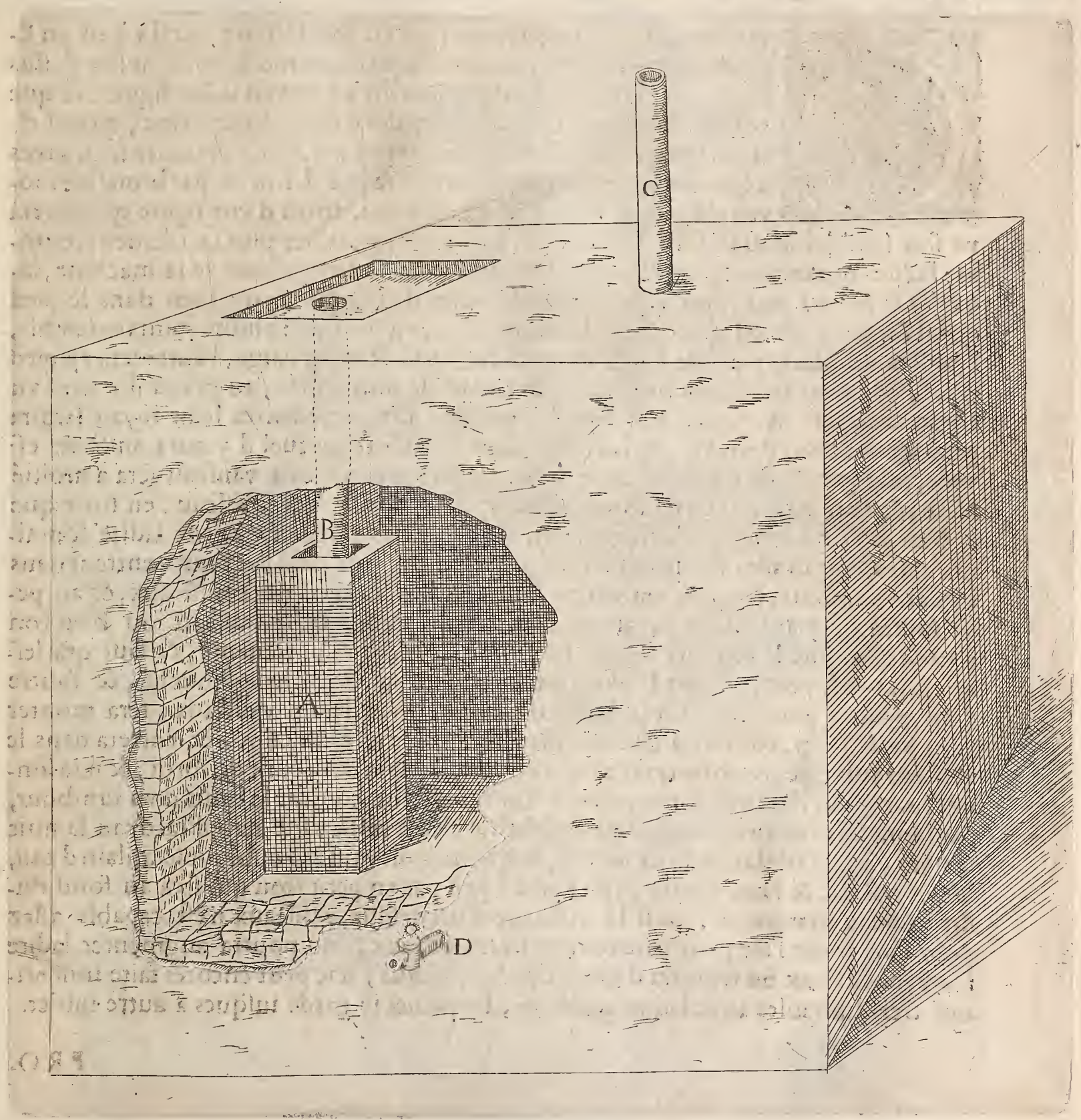




\section{Liurefecond,}

6)

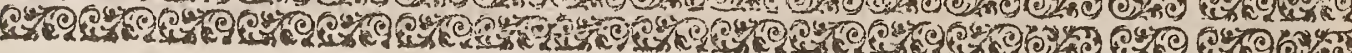

\section{P R O B E E M E X X X V.}

Pour faire vne machine admirable, laquelle eftent pofee au pied d'vne figure, iettera in fon aus lesser du Soleit, ou quand le Soleil donnera defses en forte qu'il femblera que ladite figure facs ledit fon.

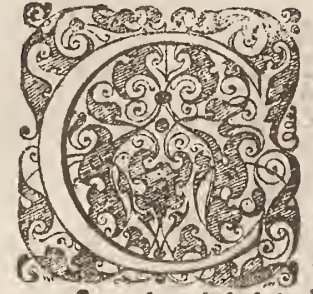

Orneille Tacite, fait mention en fon hitoire, qu'il yà eu en Egypte, vne ftatue de mennon, laquelle quand le Soleilluifoit deffus ietroit vn certain fon, Paufanias dit auoir veu ladite figure, \& que ce fon eftoit femblable, à celuy des cordes d'vne harpe, quand el. les fe rompent. Or fuiuant les trois machines precedentes traitees 6.2. vn fon femblable au fon d'vn tambour, \& pour demonftrer plus facillement, com. me ladite inuention fe peur faire, ie demonftreray la conftruction de la machine, la. quelle fe pourra puis apres adaper dans le corps de la figure, ou bien dans le pied deftal furquny elle eft pofee, foit doncques deux vaiffeaus de cuiure joints enfemble, l'vn fera de quatre pieds de long, vn pied de haut, \& vn de l'arge, l'autre fera vn pied cube, \& feront tous deux bien clos, \& foudés de tous coftés, a grand ily aura vn tuyau marqué $A$, auec vne foupape comme aux precedentes ledic tuyau feruira pour afpirer leau dembas, \& la rendre dans le vaiffeau auquel il y aura auffi vn efvent, marqué $F$. \& fera bon de le fouder ferme, quand ledir vaiffeau fera à moitié plain, \& faut quil y aye vne fontaine naturelle deffoubs ledit vaiffeau, en forte que le bout du tuyau foudé à la foupape $A$, puiffe tremper dedans l'eau de ladite fontai. ne, apres faut fouder vn fifon marqué $D$. en forte que les deux bouts entrent dans les deux vaiffeaux, \& qu'ils aprochent bien pres des fonds defdits vaiffeaux, \& au petit vaifeau il y aura deux tuyaux d'orgues pofees deffus ledit vaiffeau, ou bien lon pourra conduire le fon ou c'eft que l'on voudra auec des porteuents, \& faut que lefdits cuyaux foyent, ( fauoir te plus grand) de deux pieds de long bouché, \& lautre deux pouces plus court. Or le Soleil donnant contre lefdits vaiffeaux, fera monter l'eau par le fifon, comme a efté monfté à l'onziefme problefme, \& entrera dans le vaiffeau cubique, en forte que lair qui eft dedans, fera contraint de fortir, \& fera fonner les tuyaux, lefquels fonneront vin fon tremblant commele bruit d'vn tambour, par la mefme raifon de l'onziefme problefme, ledic vaiffeau fe remplira d'eau la nuit venane, à caufe dela frefcheur de l'air, \& quand ledic vaiffeau cubiquefera plain d'eau, le fon ceffera, \& l'eau fortira, apres peu à peu par vn petit trou qui fera au fond dudit vaiffeau marqué $C$, or fi la violence d'un defdits vaiffeaux n'eft capable affez pour faire fortir lair pour faire fonner lefdits tuyaux, l'on pourra augmenter ladite force, auec deux ou trois ou d'avantage de vaiffeaux, il fe peut encores faire inuentions trefadmirables auec ladite machine, lefquelles ie garde iufques à autre fubiet. 


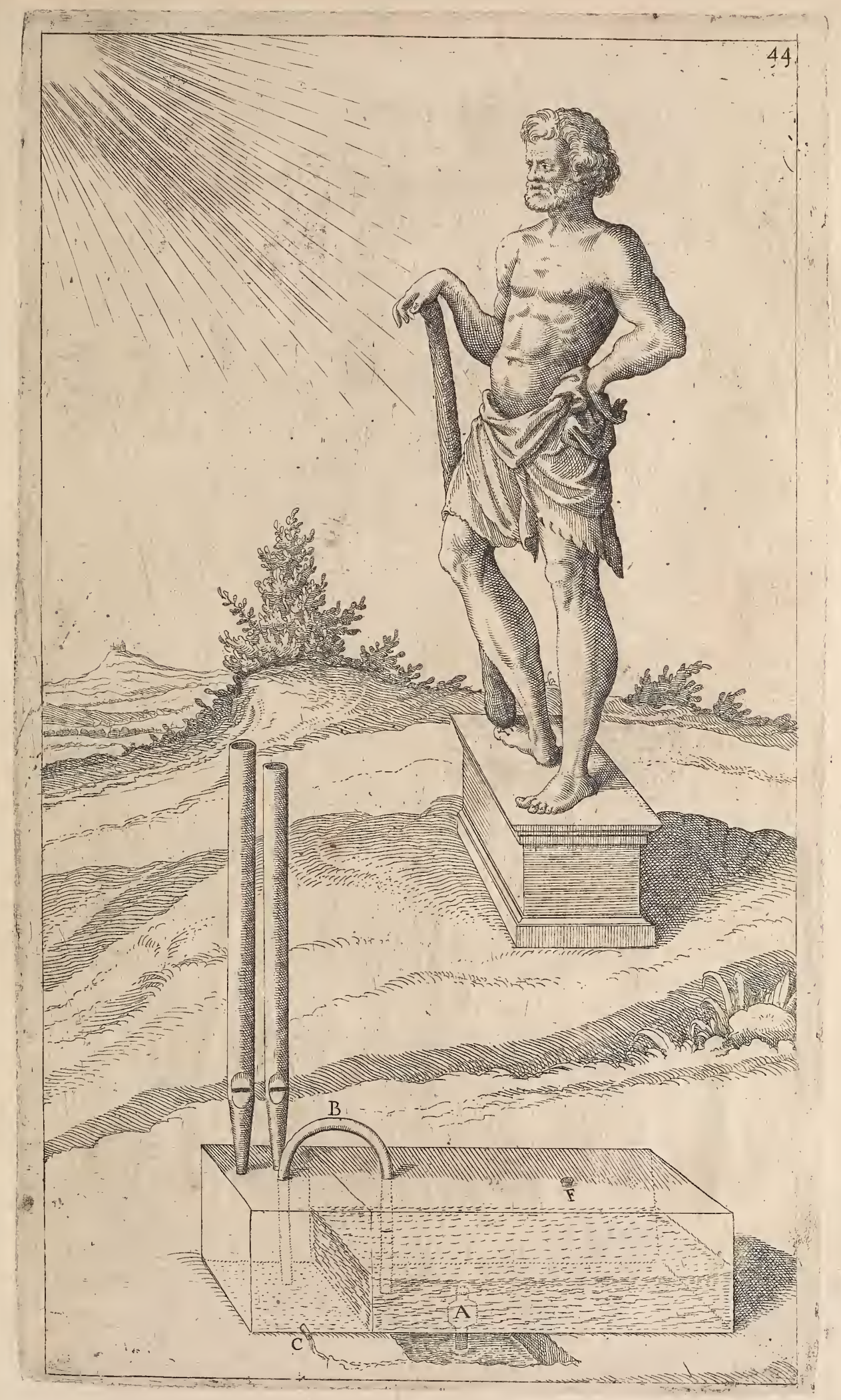




Siftefme vulgairement dit Diapafon feruar

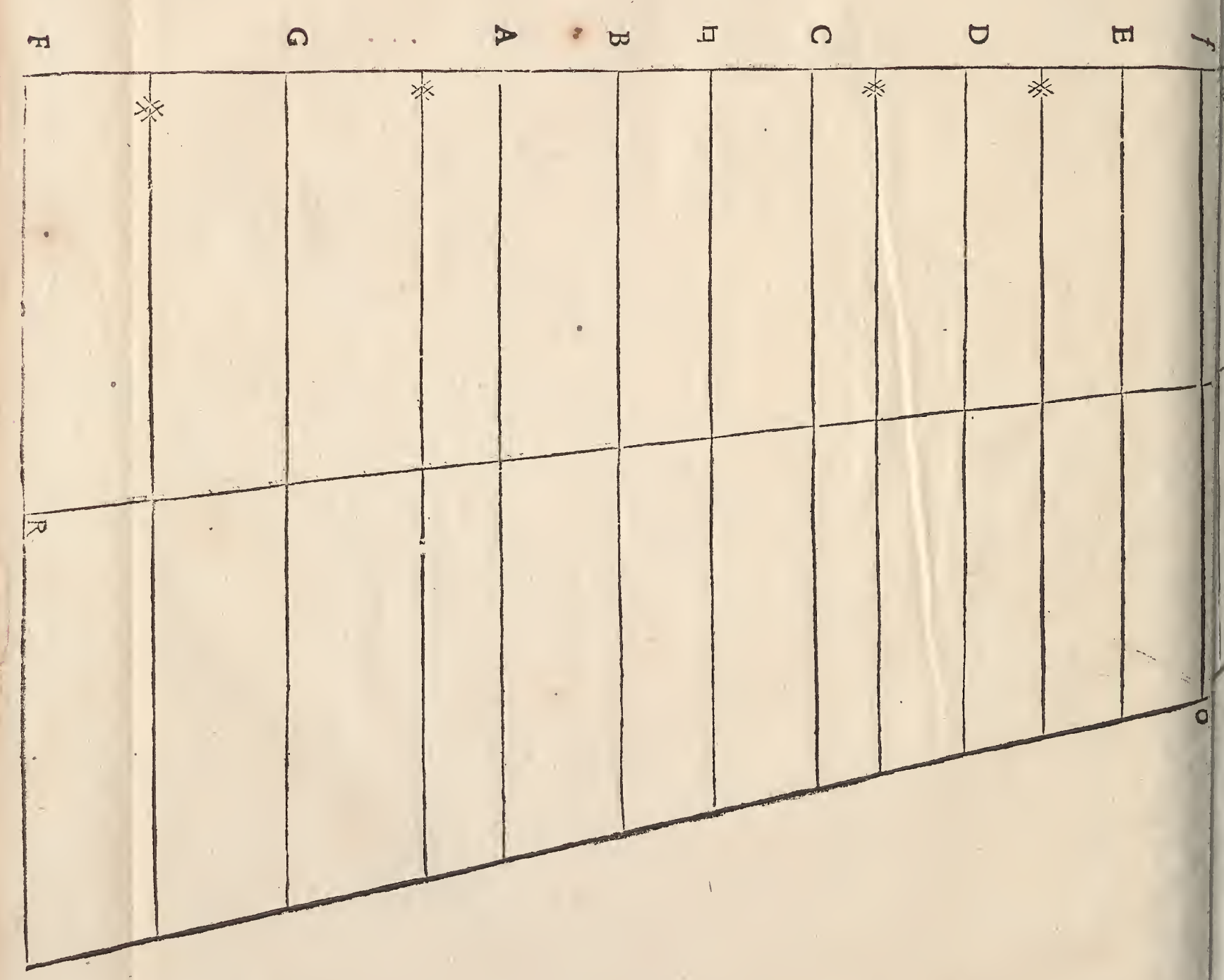


poür la mefure des tuyaux d'Orgues.

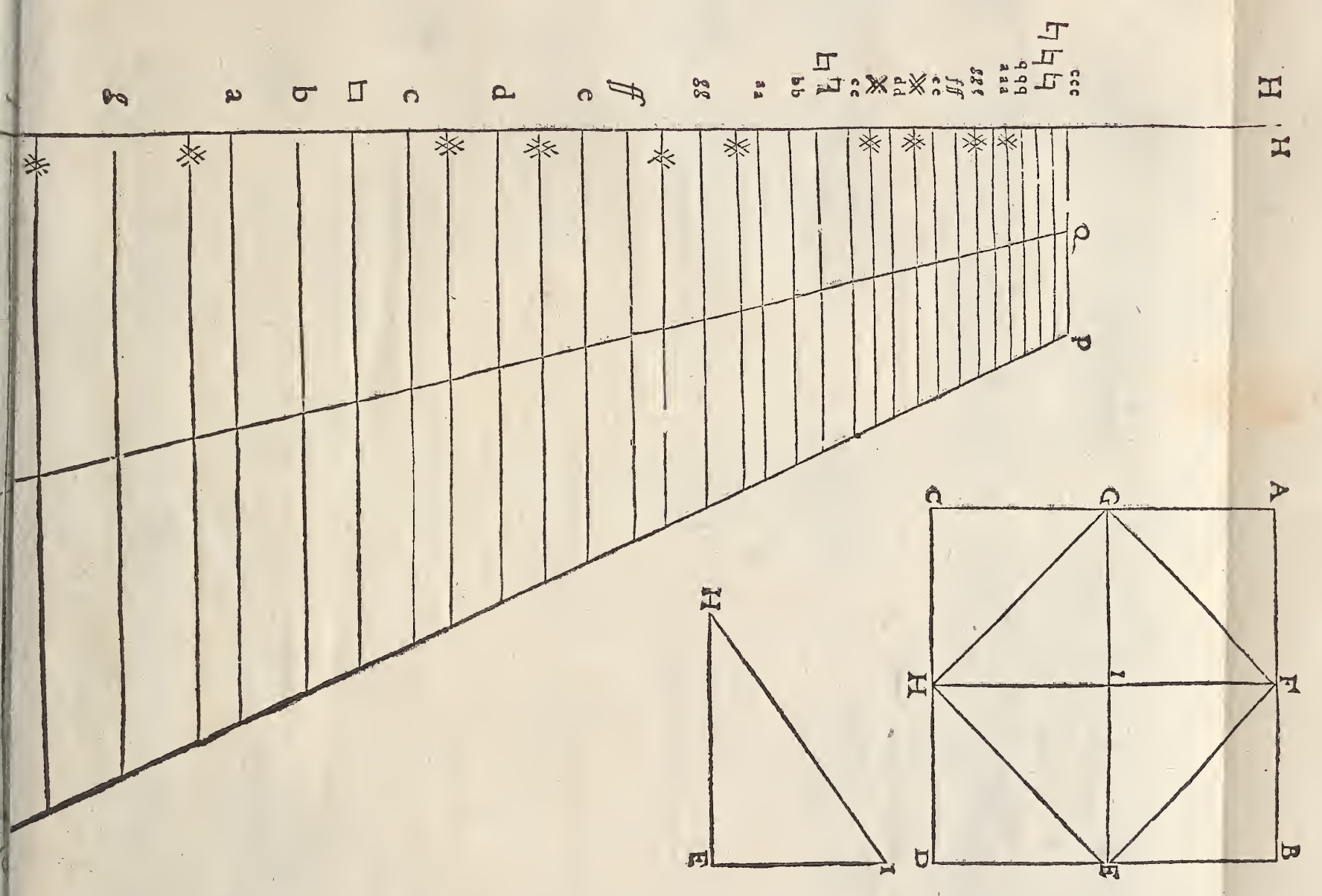





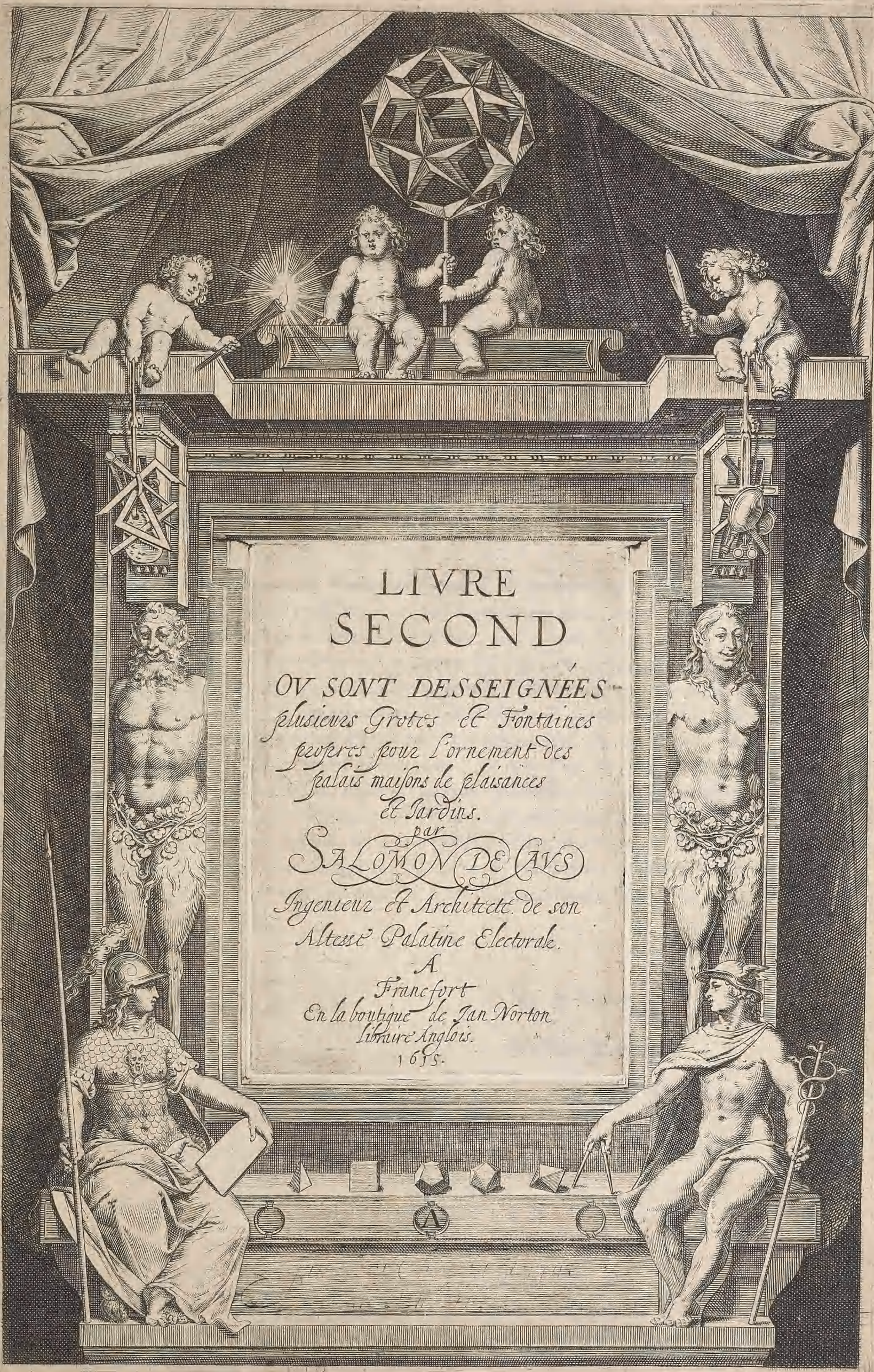


4.

is

,

1.

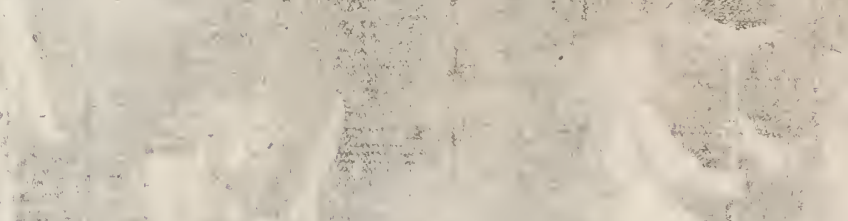

$(x+1)+\frac{1}{4}$

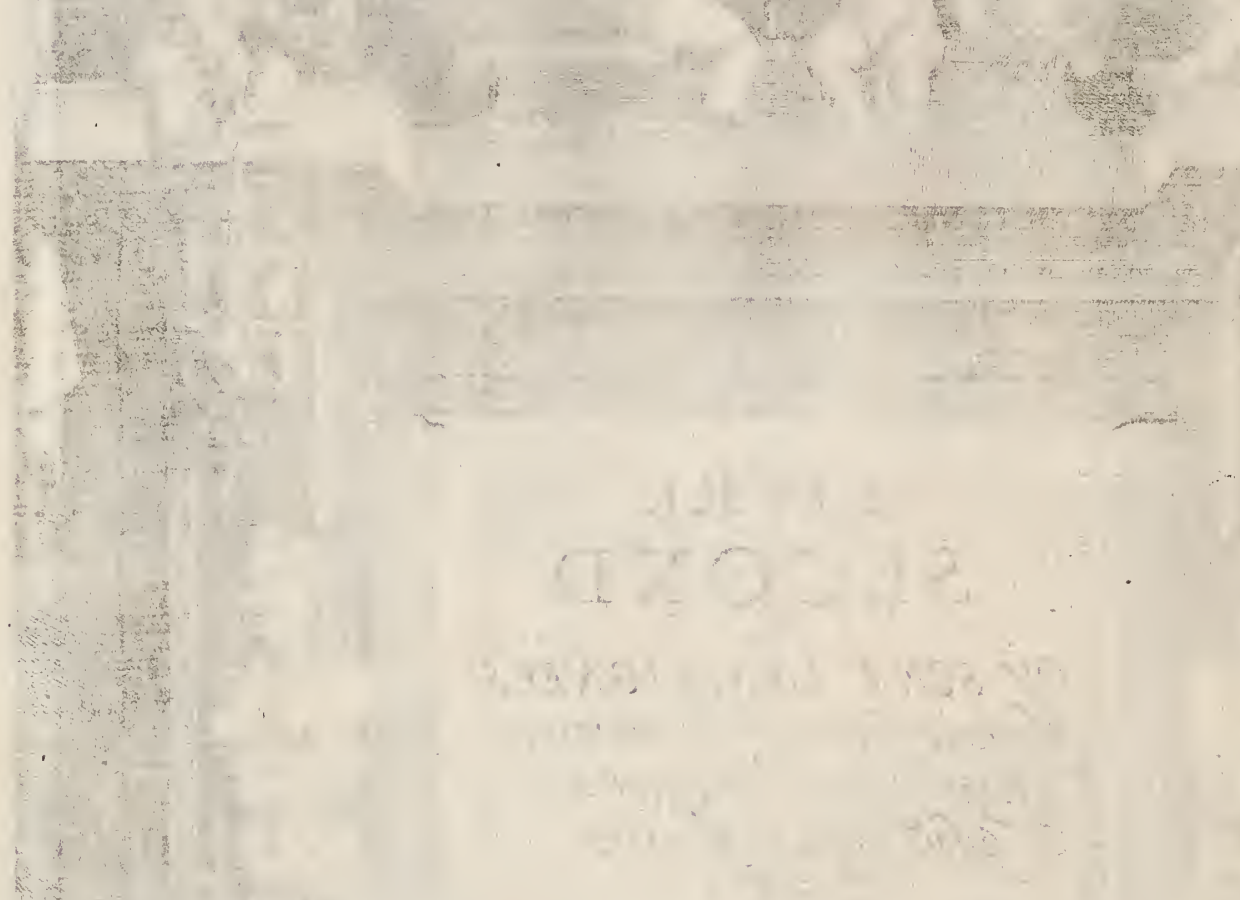




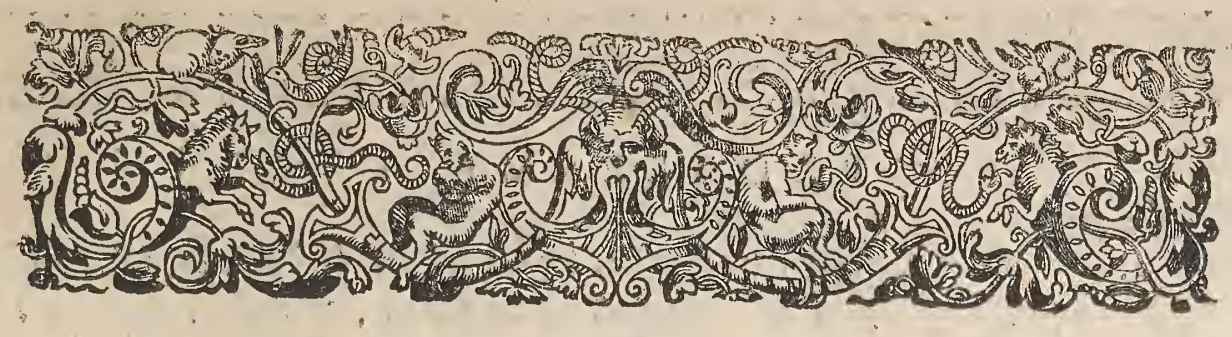

A I.A TRESILLVSTRE ET

VERTVEVSE PRINCES SE ELIZABETH,

\section{PRINCESSE DE LA GRANDE BRETAIGNE.} EECTRICE PALATINE, \&C

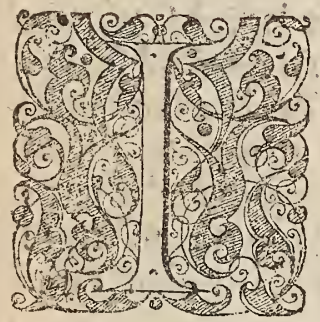

I n'est pas en moy V eriueufe Princeffe, devons prefentercbojes dignes de vos merites. CXais f̧̧achant l'aniour qu'auez porté, eg continuez de porter, à libeureufe memoire, du $\mathcal{X}$ Coble $\omega^{2}$ gentil Prince de Galles, $i$ ay reprefenté icy quelques deffeings, que j'a y astrefois faits, eftant ì fon fervice, aucuns pour fervir d'Ornement en la maifon de Richemont, Ef les autres pour latisfaire a fa gentille curiofaté, $q$ iat defiroit toufiours voir E. cognoistre quelque chofe de nouneau. Ei estant affeuré que. Voftre eAlteffe prendre de bonne part, ce qui vient de l'ordonnance de cegenereux Prince, $j$ ay perééque le dits deffeings ne pownsoy ent ejtze donnez er meilleüre main, il plairu doncques à Vostre Alteffe les accepier, non pour maquiter del'obligation que ie luy doibs, car ft petit fubject, ne le pourroùt pas faire, mais pour te moigner,

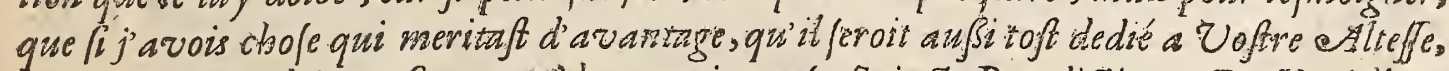
ie pre Dieu vouloir conferver, Ev luy continuer fa Sainste Benediction, Dhe Hezdelberg cepremieriour de Fanvier" 1615.

DeVofte ALtess

Lobeiffant \& humble

Serviteur S. de Caus. 


\section{Liure fecond,}

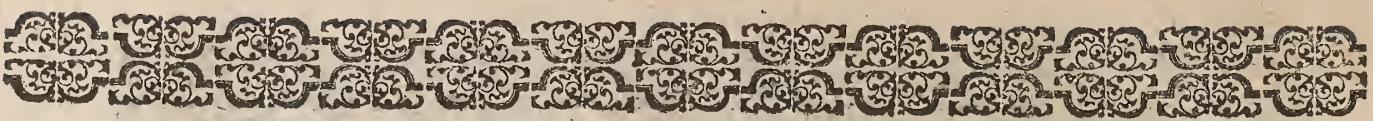

\section{P R O B LE S M E I.}

Deffeing de une grote, on il y aura vn Satire, lequel joüeraidu Flaiolet, En vne Nimphe Efcbo, iaguelle rejpondra aux cadences dudit Satire, Es outre lion paurra mettre quelques autres figures, pous jetter de l'eav.

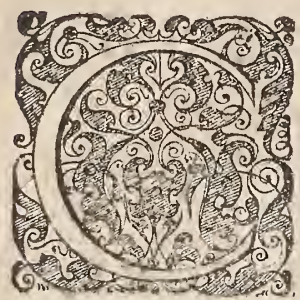

E prefent deffeing de grote, fepeut mettre dans vn Pavillon de Iardin, oubien aubout d'vne galerie, ou l'on pourra manger à la frefcheur, les deux figures marines donneront de l'eau, fçavoir lhomme par quelque poifon, ou coquille quitl tiendra à la main, \& la femme par fes mamelles, en oultre il y aura vne machine, comme (2) a efté enfeigné au vingtcinquiefme Problefme, derriere la figure du satyre, il y aura vne Nire, laquelle reprefentera le jeu d'vn Flaioller, \&à lopoficedudit Satire femblera fonner, \& ce par le moyen de quelques portevents, lefquels ferone conduits depuis la machine iufques ou fera ladite figure de Nimphe, \& feront pofez derriere icelle, \& \&audra prendre garde que les tuyaux qui reprefente ledic Efcho, ne fonne fi fort comme ceux du Flaiollet, car chatcun fait que l'Elcho ne refpond iamais fi forr, comme le fon qui le caufe, l'on pourra aufi faire defcendre des eaux, au long des Roches, pour lornement de l'ou urage, \& la table ronde qui eft au millieu du pavillon, fervira pour manger deffus à la fraifcheur, \& auffi pour faire jetter plufieurs figures d'eau par lareifice des tuyaux, qui fe pourront mettre \& aiufter fur vn autretuyau de cuiure dans le trou de ladite, table en forte que c'eft oeuure eftant bien conftruit \& ordonné aportera vne grande delectation.

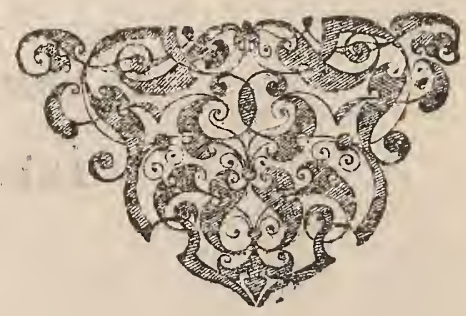




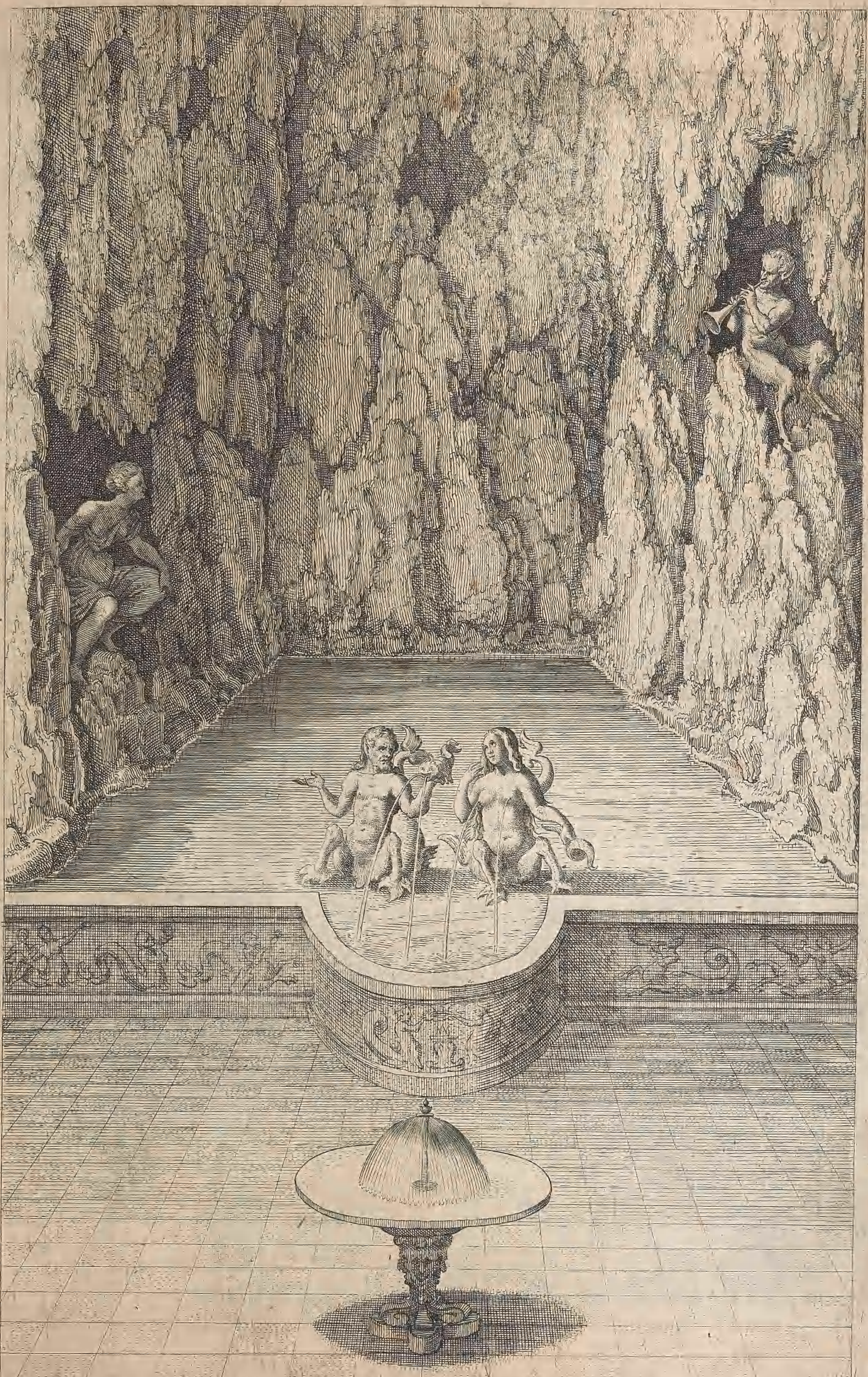




\title{
Liure lecond,
}

1. 30

\section{PROBLESME II. \\ Deffeing d'une grote ou il y a rue Balle laguelle fe lére awec la force de lears.}

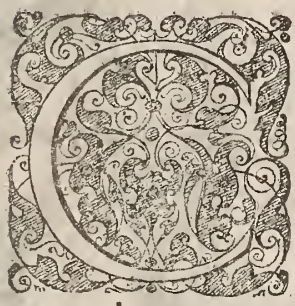

Es s autre deffeing de grote fe peut auff mettre dans vn pavillon, ou au bout d'vne gallerie, \& pour faire que l'eau efleve bien la balle fi ladite eau procede d'vne conferve, il faut que le fond de ladite conferve, foic pour le moins douze pieds plus haut quela fuperficie de la terre, \& a a plus vingtquatre pieds, le tuyau par ou fort l'eau par ou fort l'eau, fera tout au bas d'vn vaiffeau, en forme d'vn entonnoir, pour recevoir plus facilement ladite balle, quand elle tombe, \& pour efvacuer leau qui tombe dans ledic vaifeau, il y aura des trous tout au bas d'iceluy, lon pourra orner la Roche, auec quelques animaux faits de coquilles naturetles accommodees, \& cimentees enfemble, lefquels ietteront de l'eau par des petits tuyaux, qu'ils auront dans la bouche, en forte que lefdits jets, puiffent donner quelque fois contre la balle pour la faire tomber. \& tacontinent elle fe releuera par le moyen de l'eau, qui la repoure en haut, \& ainfi fautelant elle donnera du contentement à la veuie, mais faur noter, que pout bien voir le brifement de l'eau, contre ladite balle,il faut que la feneftre foic oppore au midy a celle fin que le Soleil donnant, les rayons \& brifements de l'eau, contre ladise balle, fe puiffent mieux voir, \& donter conten: tement à la veüe.
\end{abstract}

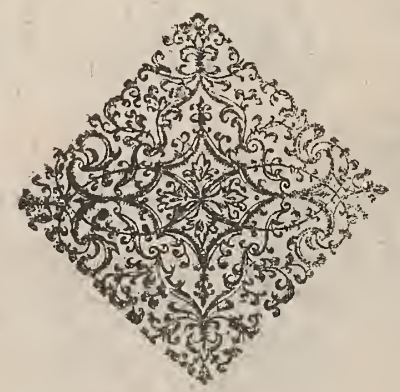




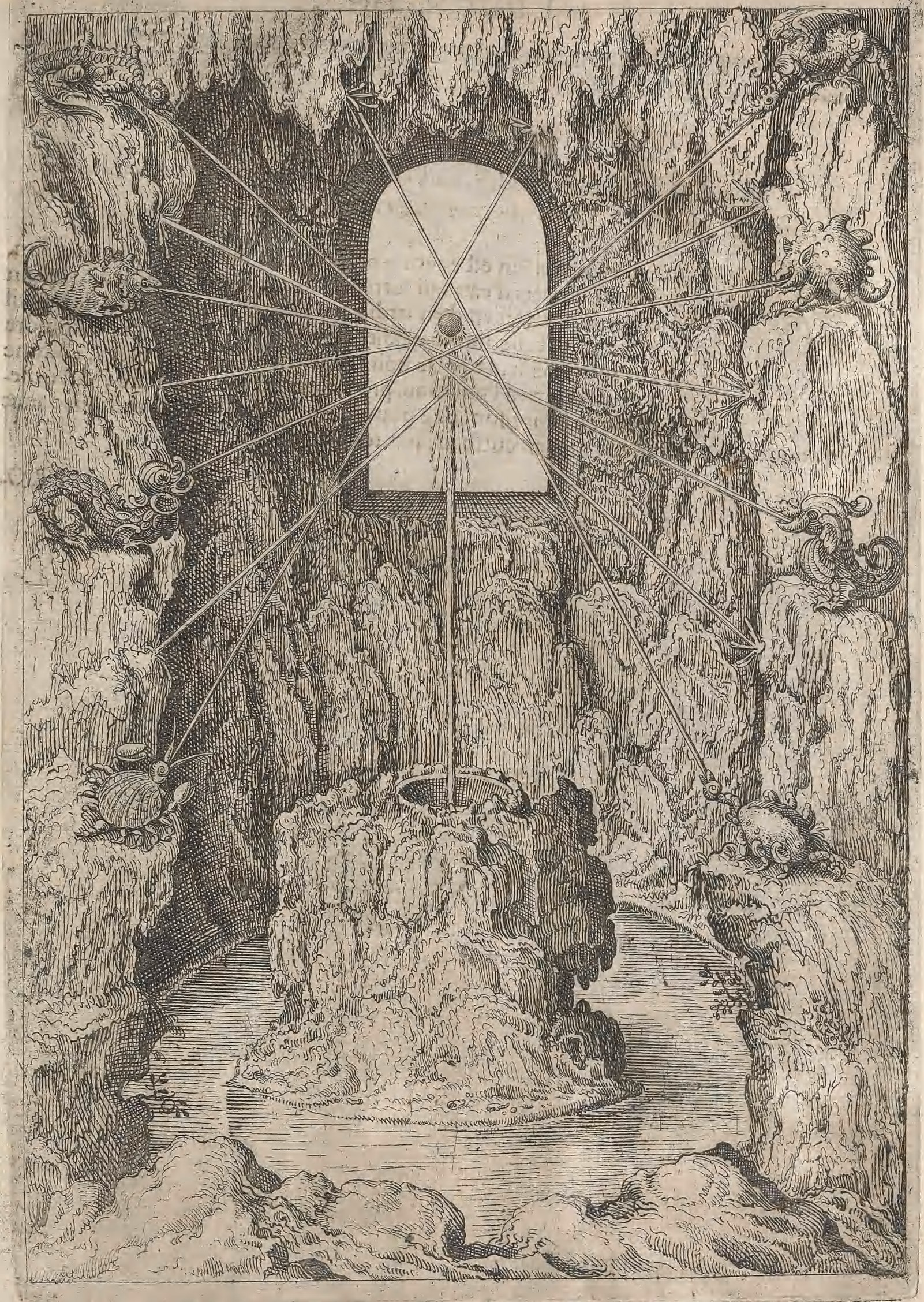




\section{Liure fecond,}

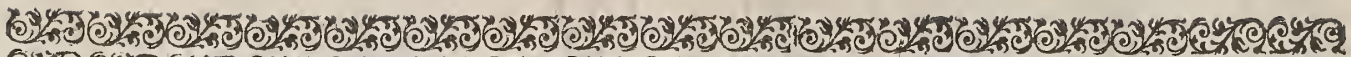

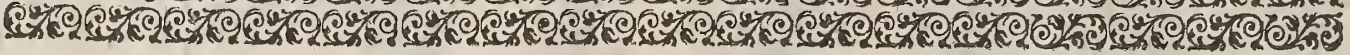

\section{P R O B L E S M E III.}

Deffein de la fontaine du cupidon, ou il y aura une tourterelle qui boira austant deau, comme on luy donnera.

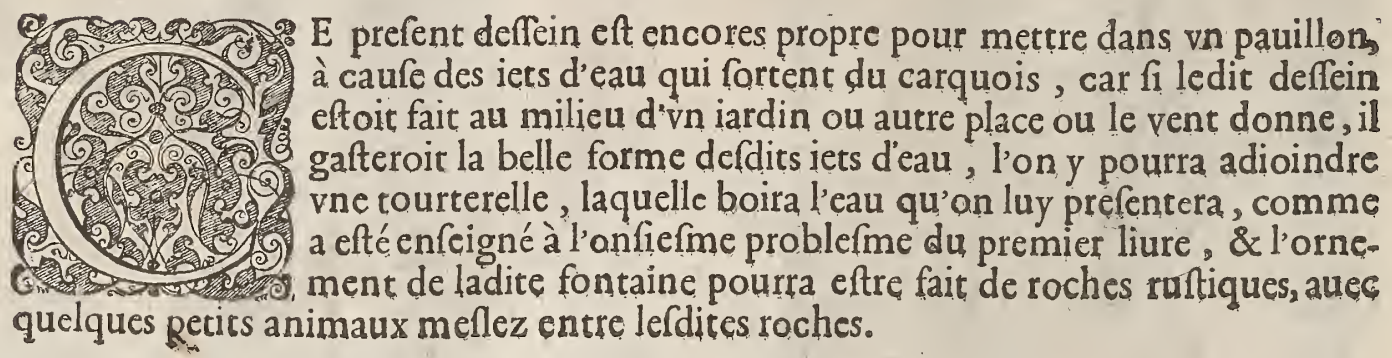

PRO.

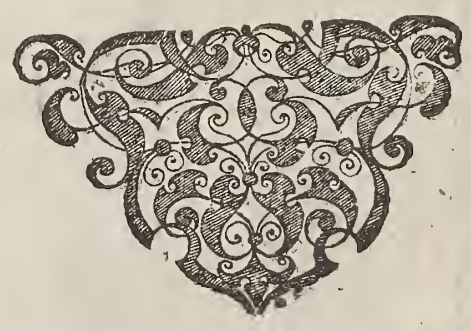


तi $\mathrm{P}$

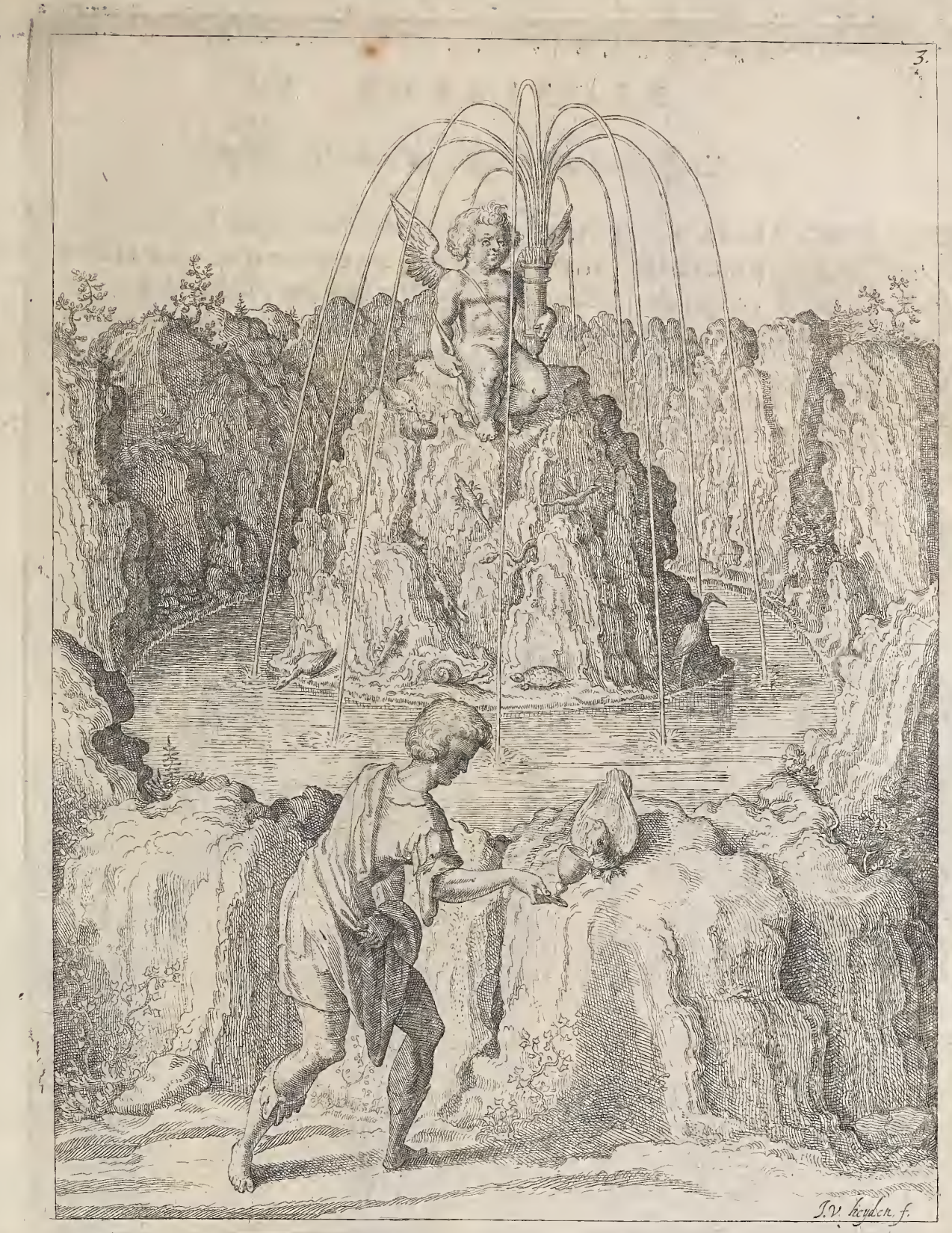




\section{Liure fecond}

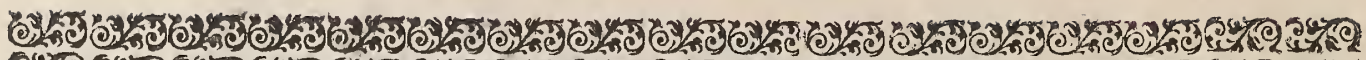

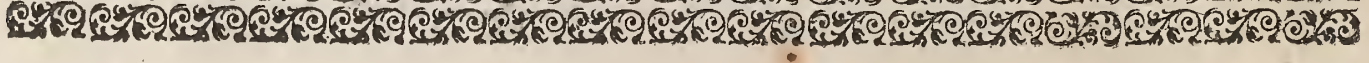

\section{PROBLES M E IIII.}

Deffeing d'une fontaine d'ordre $R$ uftique.

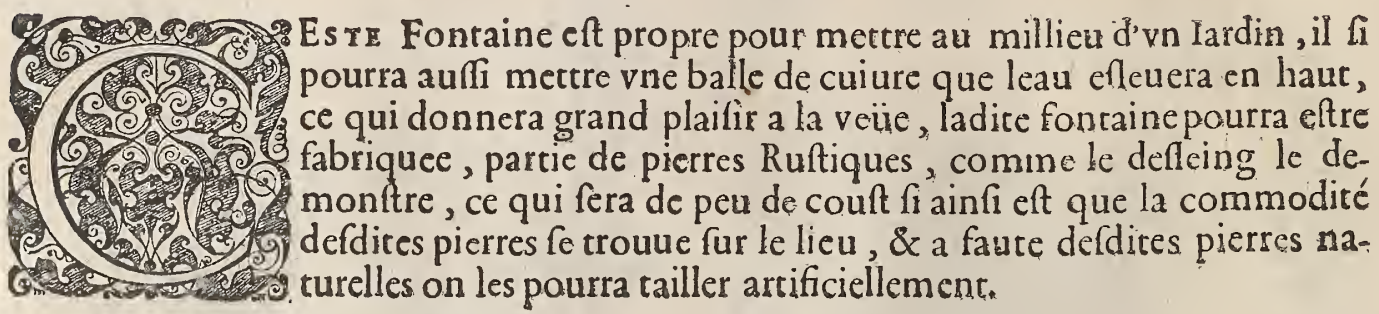

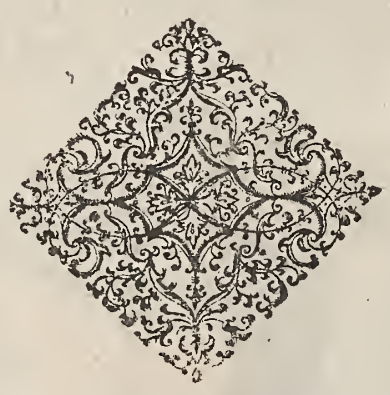



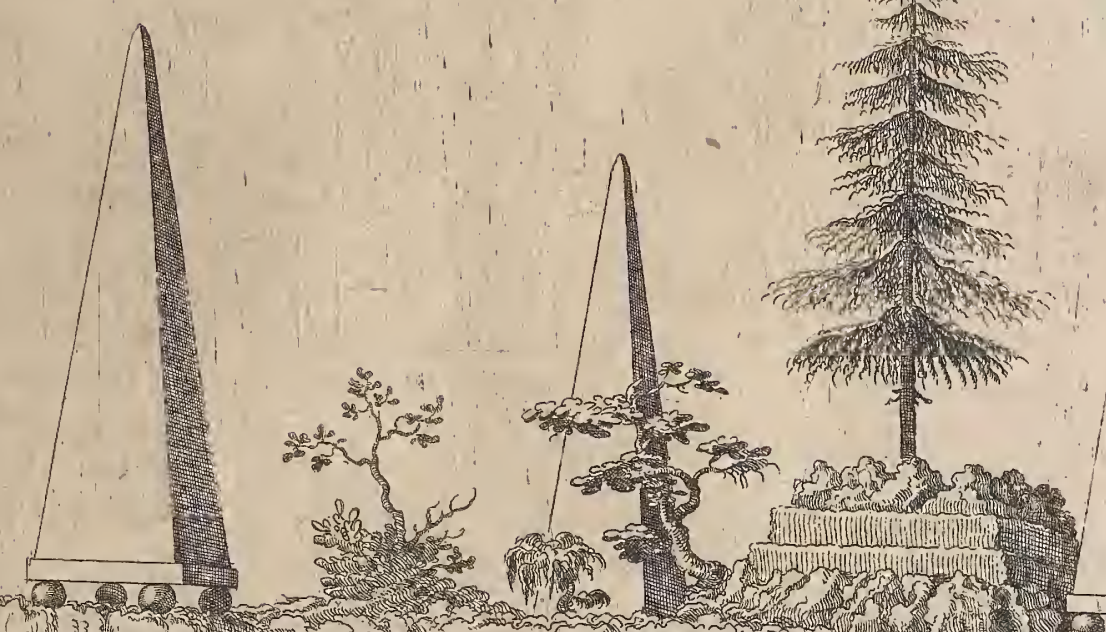

196. T.t. Mn (1)

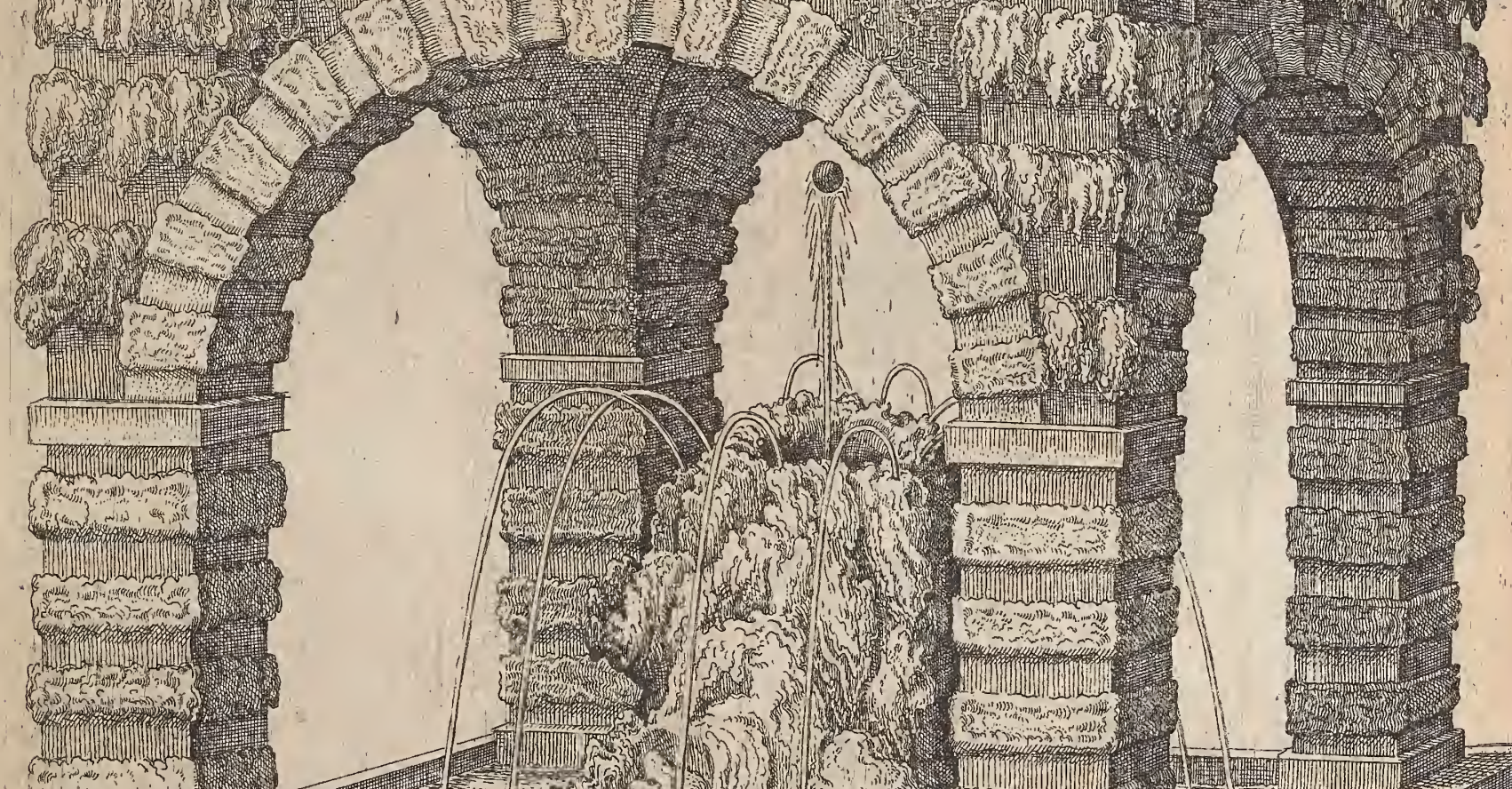

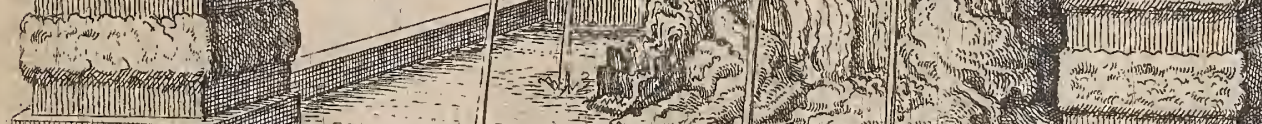




\section{Liurefecond,}

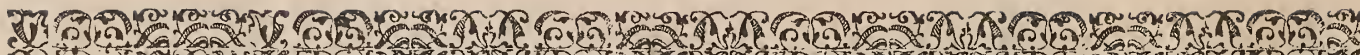
20.

\section{P R O B L E S M E V.}

Autre deffeing de fontaine pour reprefenter on Flewue, ois $R$ intere,par vne figure.

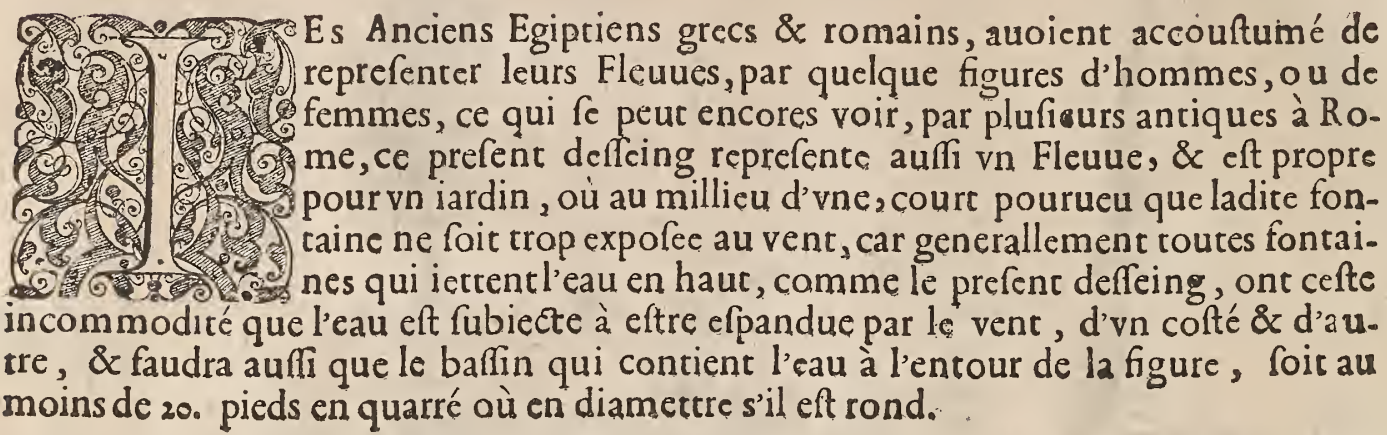

P R O.

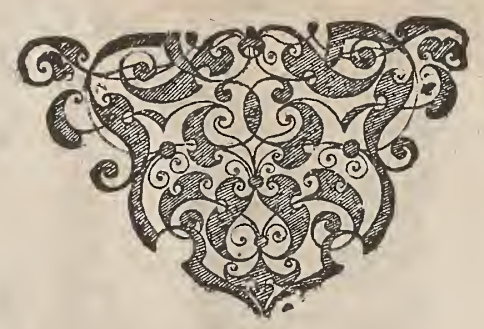




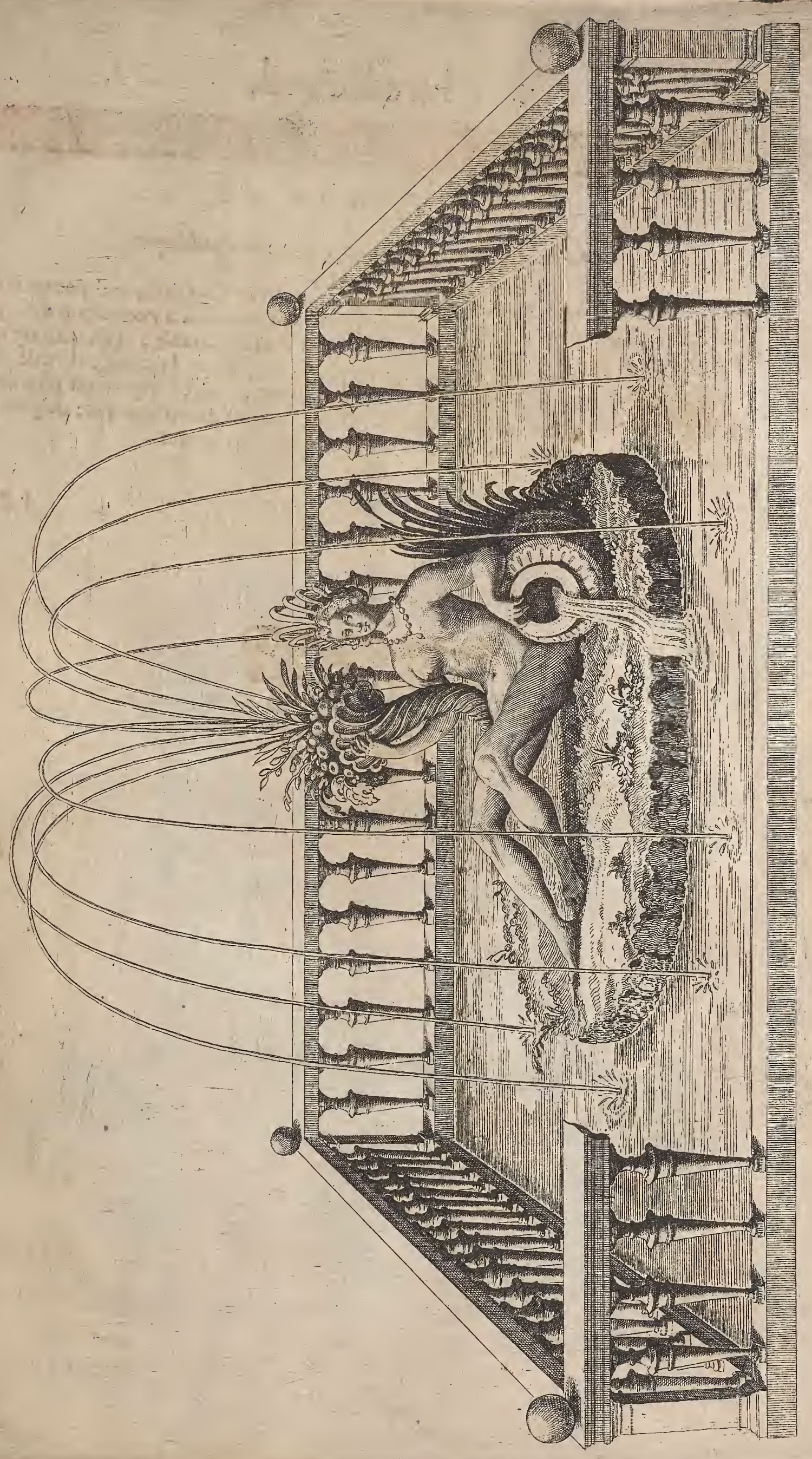




\section{Liure fecond,}

W"s, 1.

P R O B L E S M E VI.

Autre deffeing de fontaine, pour vne place publicque.

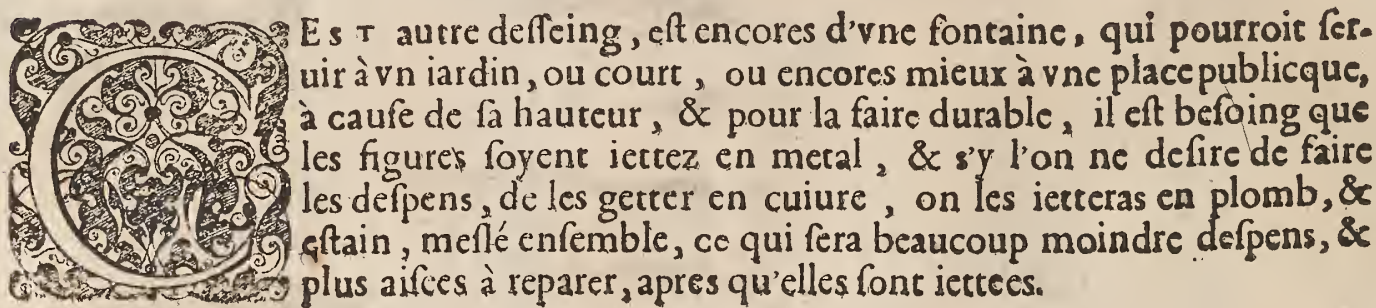

P 20.

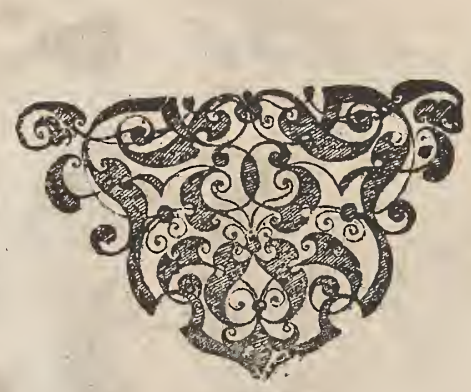




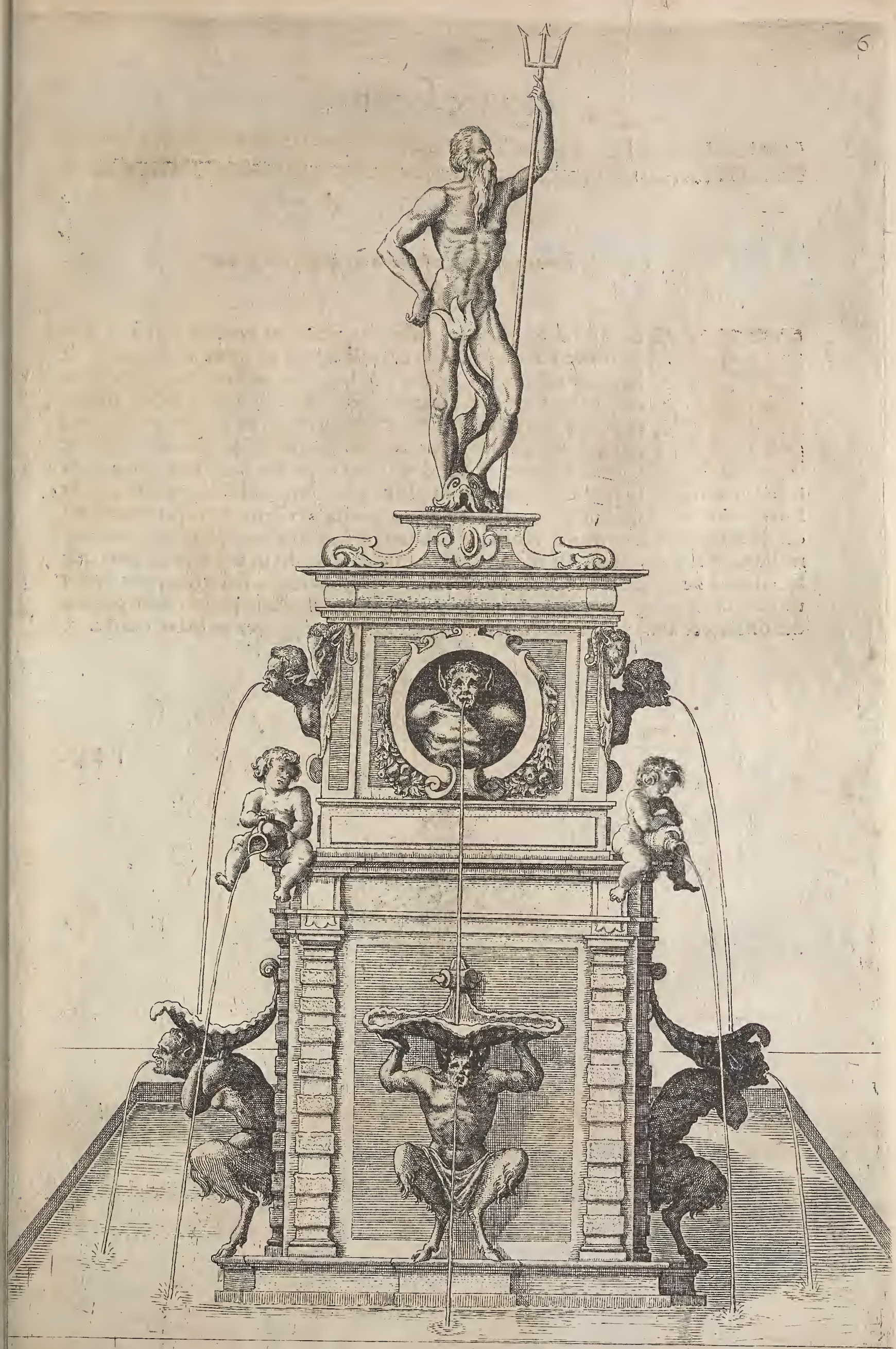




\title{
Liure fecond
}

6.月.

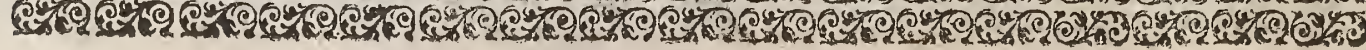

\author{
P R O B L E S M E V II.
}

\section{Deffeing d'vne volicre a oifeaus auec quelques grotes dedans jcelle.}

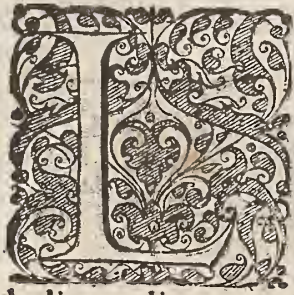

Es grotes \& ouurages ruftiques vięnnent encores fort à propos dans vne voliere à aifeaus ce deffeing icy eft d'vne de 80 . pieds de long par dehors \& vingtdeux de large par dedans l'ingnogratie \& ortografie font deffeignez icy deffoubs, \& à celle fin de mieux comprendre l'ordonnance de ladire voliere ien ay fait vn deffeing d'vne partic en plus grand volume par ou fe peuc comprendre le refte, a lopofite de larc du millieu fe pourra faire vne grote dans ladite voliere, ou les oifecaux prendront du plaifir a faire leur nids alentour \& efleuer leurs petits, \& à lopofite des autres arcades lon pourra y faire quelque petits bocages defpines blanche $\&$ autre abriffeaus, la counerture fera faite auec plufieurs ounertures de 7, ou 8. pieds en quarré chacuñ, acommodées auec du fil de laton en forre que les oifeaux ne puiffent paffer à trauers \& lefdites ouuertures feruirsnt pour laiffer tomber la pluye dedans ladite voliere laquelle eft fort neceffaire paur la çanferuation des oifeaux \& auffi pour arroufer les abrifeaux qui feront plantęz en ladite voliere.
\end{abstract}

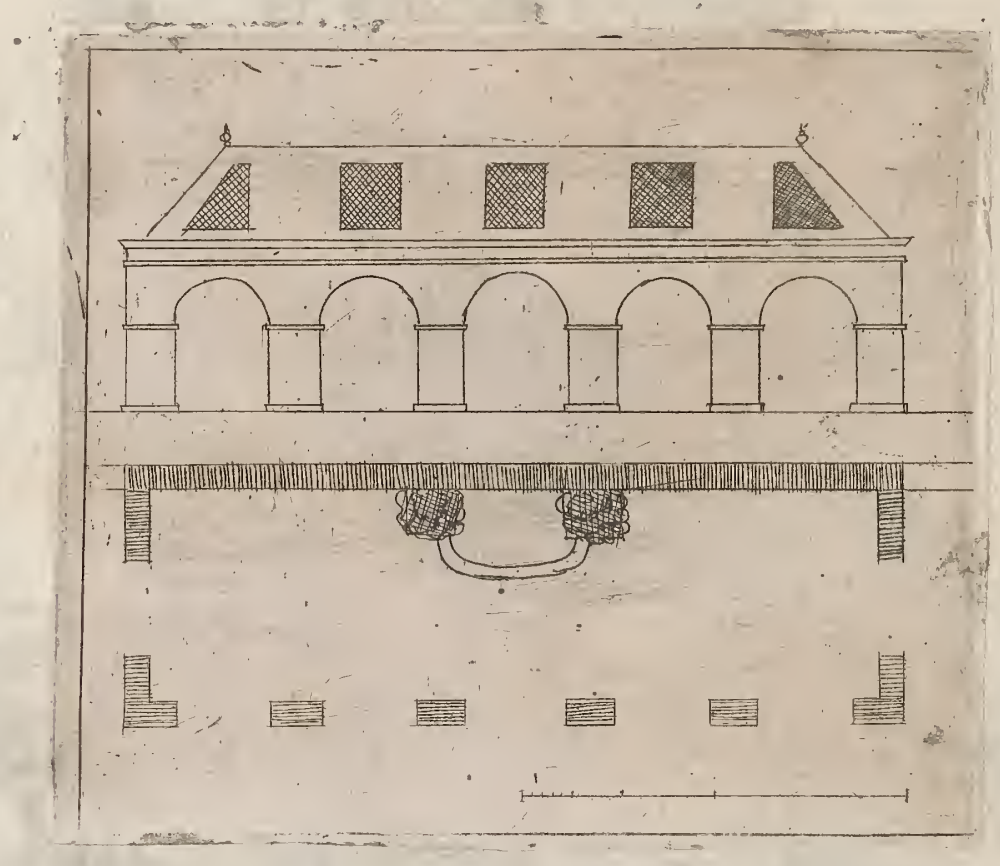

P R O. 


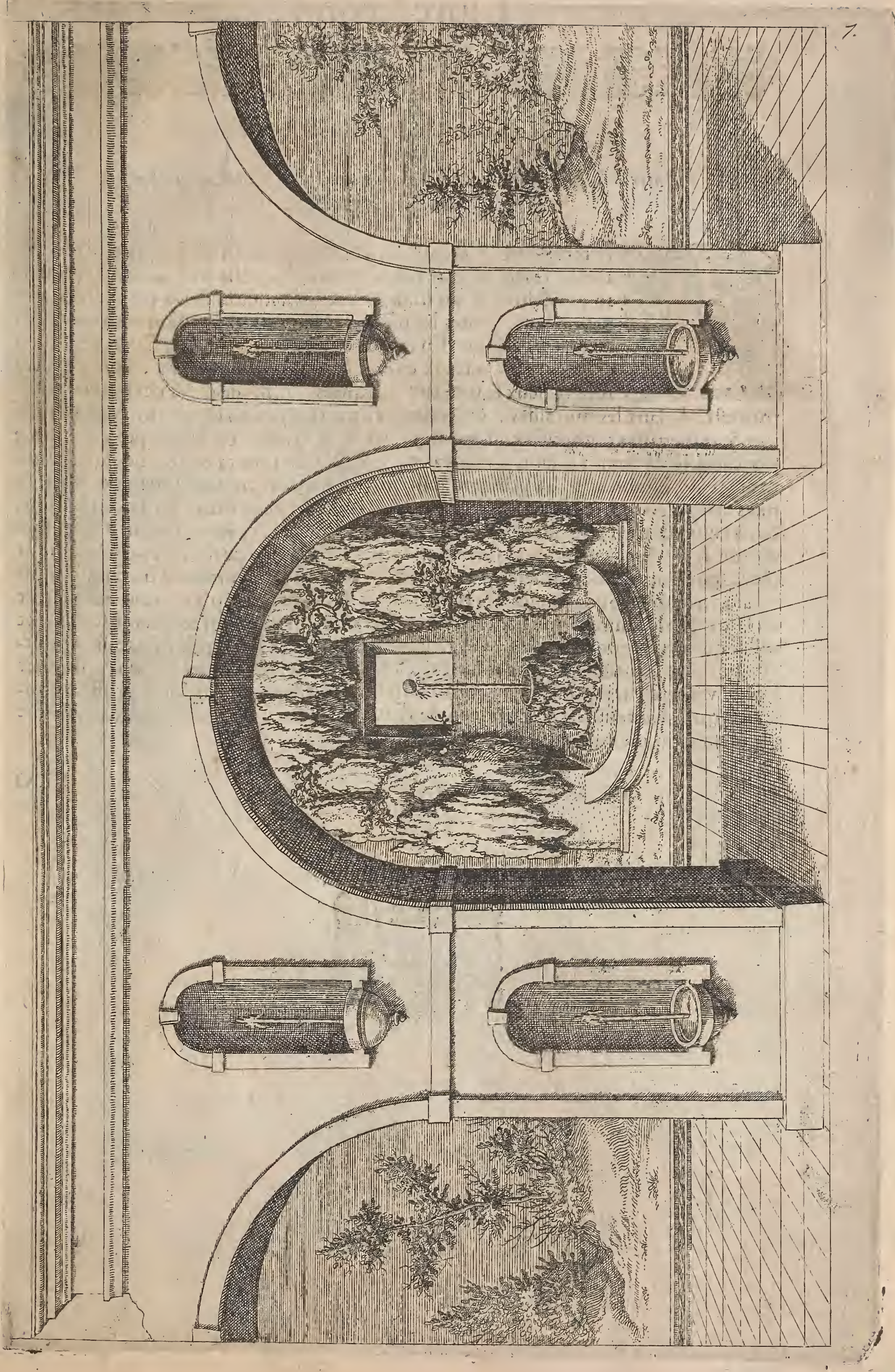




\section{Liure fecond,}

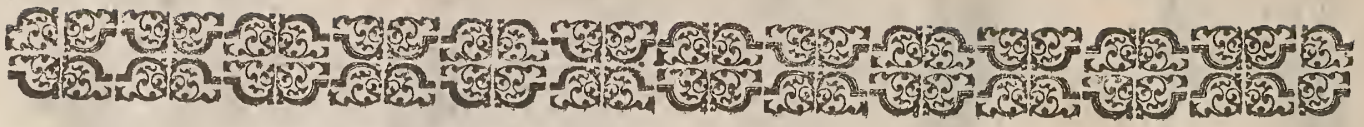

\section{PROBLES M E VIII.}

\section{Autre defeing dine autre volliere à oifeaux plus grande, accompagnec d'on parillon ans millien.}

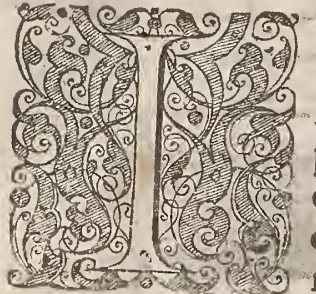

E reprefenteray piemierement les plans, tant de l'ingnografie com. me de l'ortografie dela dite volliere l'aquelle aura 84 .pieds en quarré par dehors \& au millieu fera vin pauillon de 30 pieds en quarré par dedans, toutes les murailles tant dudit pauillon comme celles de dehors auront deux pieds \& demy en groffeur, fi l'on veut bien conferver les oifeaux contre la froidure de l'hyuer, l'on y pourra Dettre deux fourneaux marquees A.B. dont les cheminees pourronteftre dedans les murailles, \& lhyuer venant lon pourra fermer toutes les feneftres \& ouuertures comprifes en lefpace C.D.E.F. G. H. en forte que cefte place foit capable de retenir quanticé d'oifeaux, \& auffil'on y pourra mettre quelques abriffeaux tranfportables qui ne peuuent auffi endurer froidure comme Orangers, Citronniers, Figuiers, \& autres tels abriffeaux defquels l'on peut orner vn Iardin en Efté, $\&$ en Hiuer l'on en pourra orner ladite volliere, \& faut faire en forte que les feneftres du toit fe puiffent ouurir quelquefois en Hiuer, à celle fin de donner air, \& que la pluye puiffent tomber fur le rdits abriffeaux \& oifeaux, \& au millieu du pauillon, il y aura vne table pour manger à la fraifcheur en Efté, \& s'y l'on s'en veut auffi feruir en Hiu er, lon pourra clorre toutes les ouuertures.dudit pauillon referuant feulement celles qui regardent les fourneaux, tellement que par ce moyen, ledit pauillon pourra aufli eft re efchauffé, defdits fourneaux, \& s'y l'on veut faire les defpens, d'orner ladite volliere auec quelques roches naturelles, mếmement quelques artifices d'oifeaux, qui chanteront pàr le moyen de l'eau comme à efté enfeigné aux difiefme problefme du premier Liure. 

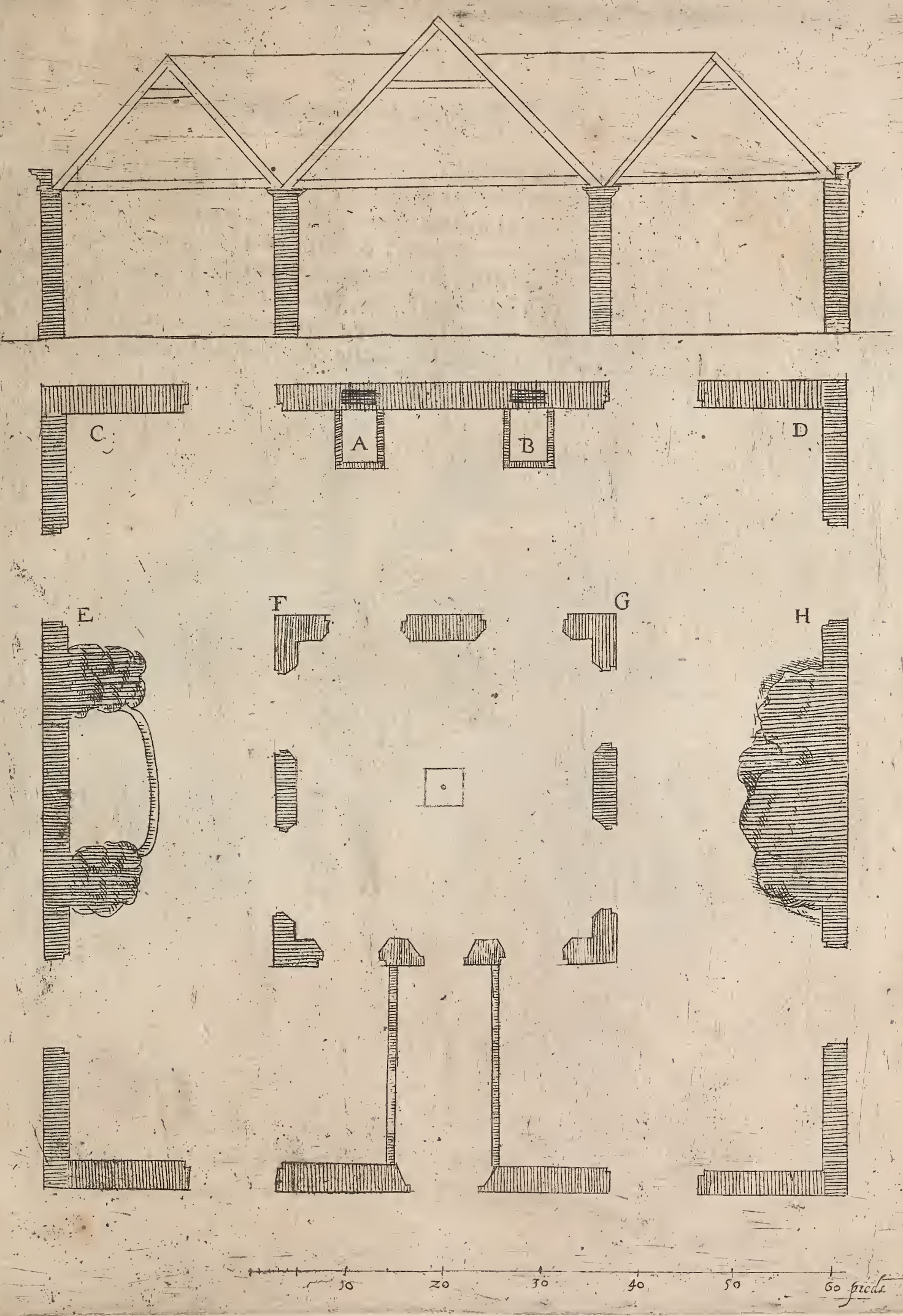


\section{Liure fecond,}

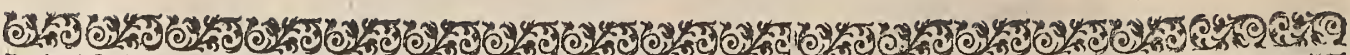
б*

\section{P R O B L ES M E I X.}

\section{Plan prepectif du precedent defeing.}

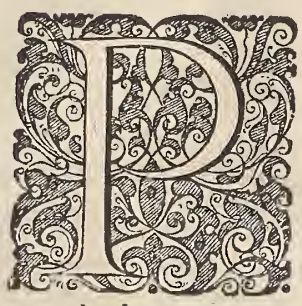

AR ce plan perfectif lon peut comprendre facillement l'ordonnance du precedent deffeing, parmi les roches ou pierres ruftiques lon fera plufieurs trous grands \& capables pour les oifeaux, à faire leurs nids dedans, \& auffi lon plantera forces abriffeaux defpines blanches, tant aux enuirons defdites roches comme des murailles, lefquels feruiront auffi pour c'eft effect, la couuerture (a) en partie reprefentee, aueciles ouuertures de treilles de fil de fer f̧̧eu eftre veu.

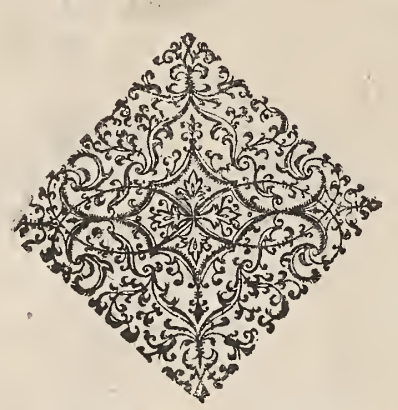




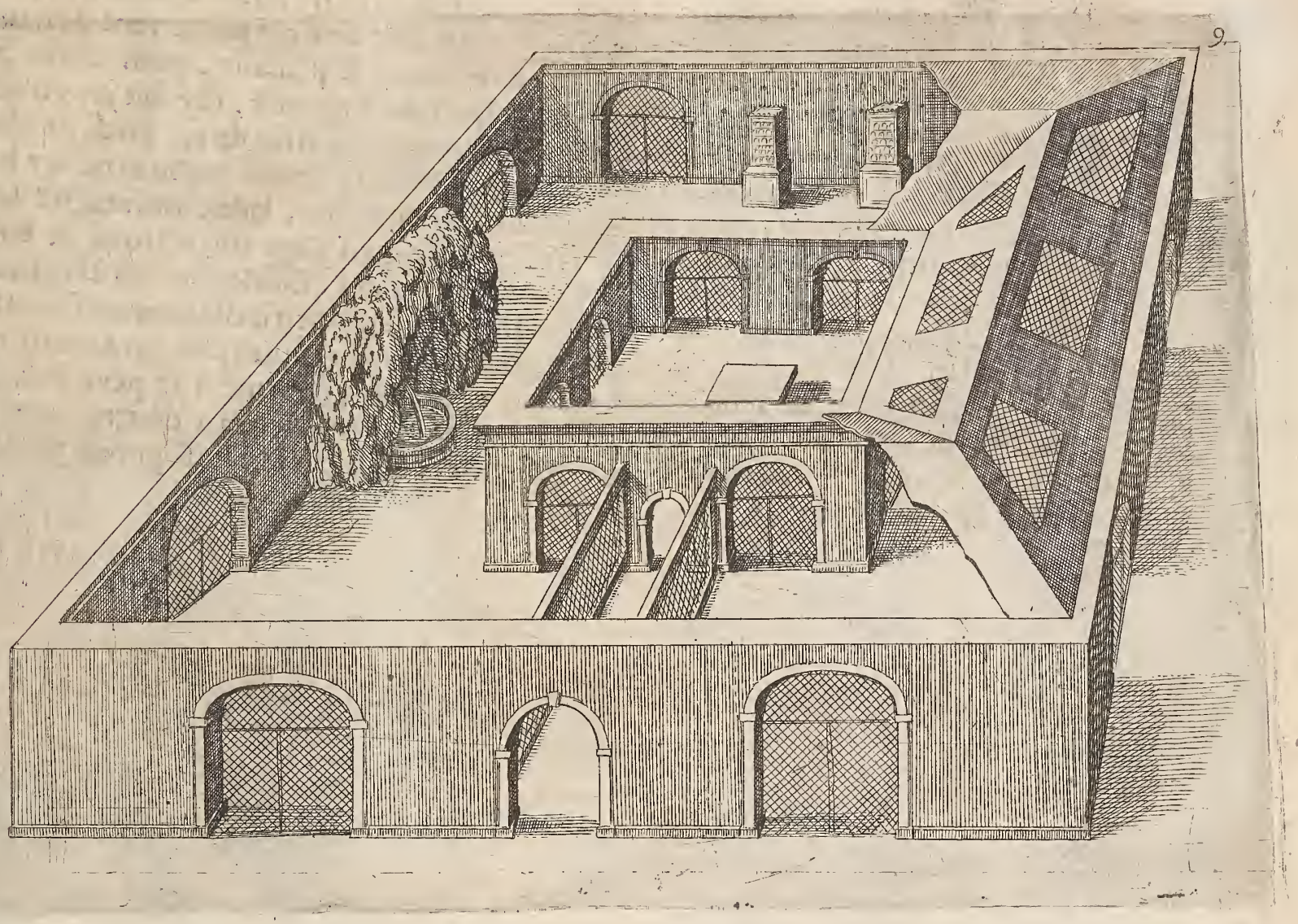




\section{Liure fecond,}

(อ)

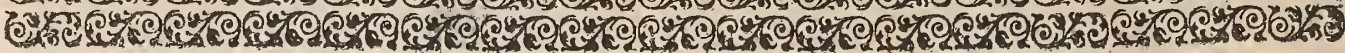

PR O BLES M E X.

\section{Deffeing dive mantagne au millieu divn lardin awee quelques grotes dedans.}

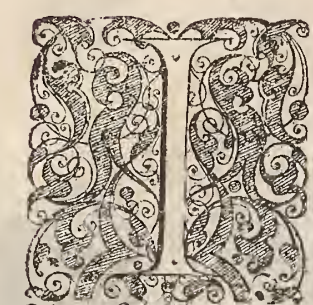

L y à plufieurs beaux \& excellents iardins fituez en planure, de forte quil n'y à moyen d'en voir la forme, ny les parterres contenues en iceux \& \& me semble que l'afpect le plus beau d'vn iardin eft deftre veu d'enhaut, c'eft pourquoy ie fuis daduis que pour aider à ce defaut quand lefdits iardins ne font point veus de haut, de faire quelque ouurage haut efleué \& plaifant, pour eftant au haut diceluy auoir mieux l'afpect des parterres, iay fait icy vo deffeing fort propre pour vn tel iardin, c'et vne montagne quarree de 84. pieds de cha. cun cofté \& enfeuee de $\$ 5$. pieds iufques en haut le plan de ladite montagne icy bas deffeignés en perite forme, \& le plan perfpectif va fuilant, ladice montagne fera faite de mafonnerie de pierre tout à l'entour, en forte qu'il y aye force trous \& concauitez par dehors pour mettre de la terre pour planter des arbriffeaux tour à l'entour, il y aura vn chemin pour monter au haut, tournant à l'entour d'icelle commeil fe peut voir par le plan, le dedans fera voulté \& y pourra l'on faire quelques grotes qui receuront lumiere par deux feneftres au deffus de la porte, comme il fe peut voir au deffeing \& tout au fommet de ladite montagne s'y lion veut, lon y mettras vne figure laqu'elle fonnera vn fon au leuer du Soleil , comme à efté enfeignéau pernier problefme du premicr liure 


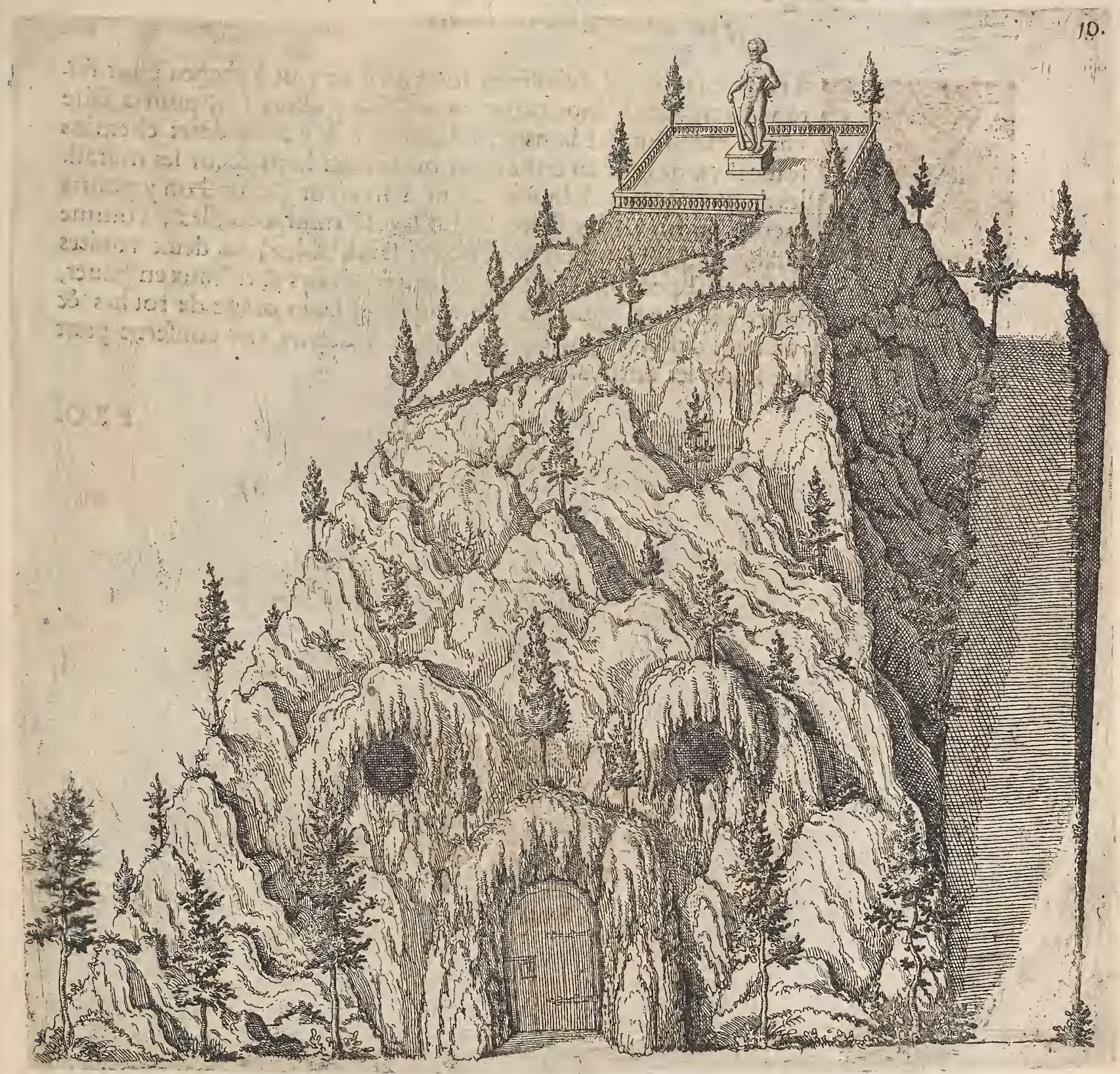




\section{Liure fecond,}

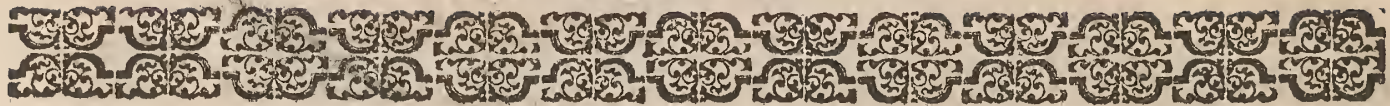

\section{P R O B L E S M E XI.}

\section{Deffeing divne haute terraffe accompagnee dequelgues grottes pour mettre dans on lardin.}

1. A fi le iardin eft difpofé en forte quil ne vint à propos pour fai-

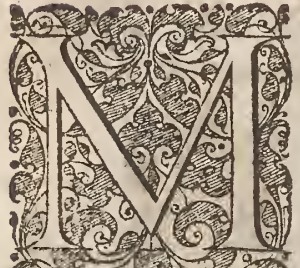
vne terraffe fuiuane le prefent deffeing, \& y aura deux chemins fçauoir vn de chacun cofté pour monter en haut $\&$ fur les murailles defdits chemins lefquels feront à hauteur dapuy l'on y pourra mettre de toutes les fortes d'abriffeaux tranfportables, comme Orangiers, Citronniers, \& autres femblables, les deux voultes au deffoubs de ladite terraffe posuront feruir pour mettre lefdits abriffeaux en Hiuer, $\&$ au haut de ladite terraffe lion y pourra faire quelques grottes ornez de roches \& artifices d'eaux, \& au haut defdites grottes l'on y pourra mettre vne conferue pour tenir l'eau, pour faire joüer les artifices defdices grottes, 


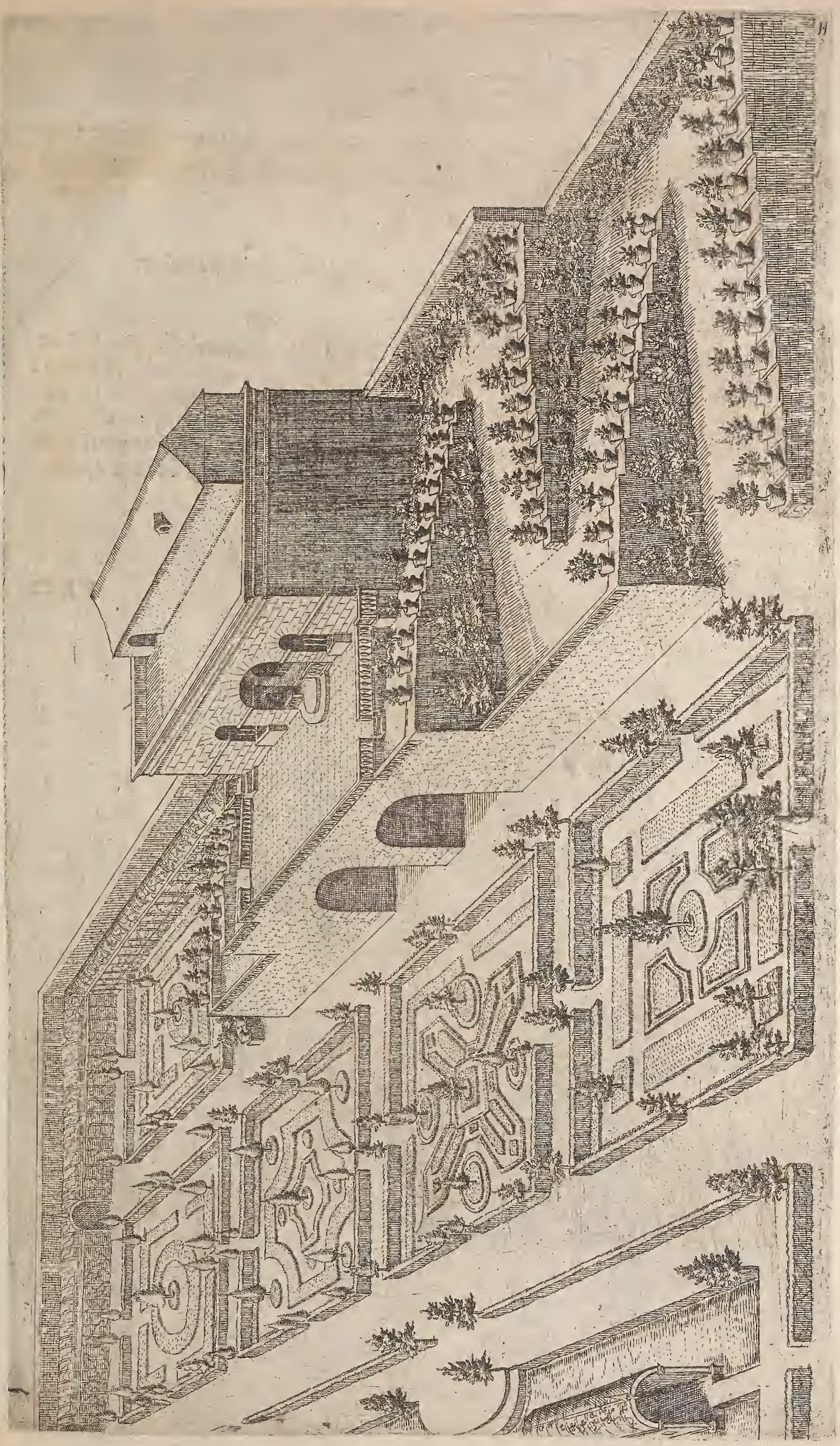




\section{Liure fecond,}

ว. 2.

\section{PROBLES M E XXII.}

\section{Deffeing disfrontispice de la grote fituee furla terraffe du precedens deffeing.}

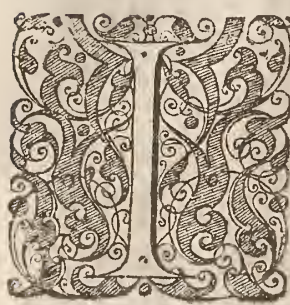

A y mis icy vn deffeing en plus grand volume pour compren drel'ordonnance du dehors de la grotte du precedent deffeing, le dedans pourra eftre de douze ou quinze pieds de large, quarante ou cinquante de long, en forme de galerie, ou mefme lon pourra mettre des arbriffeaux d'orangers \& Citronniers en hyver pour eftre gardez de la froidure, \& a uffi feruira d'ornement a ladite grote,

I R O.

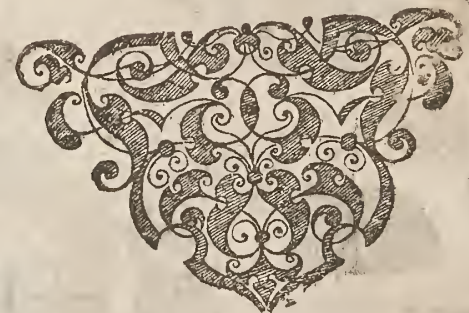




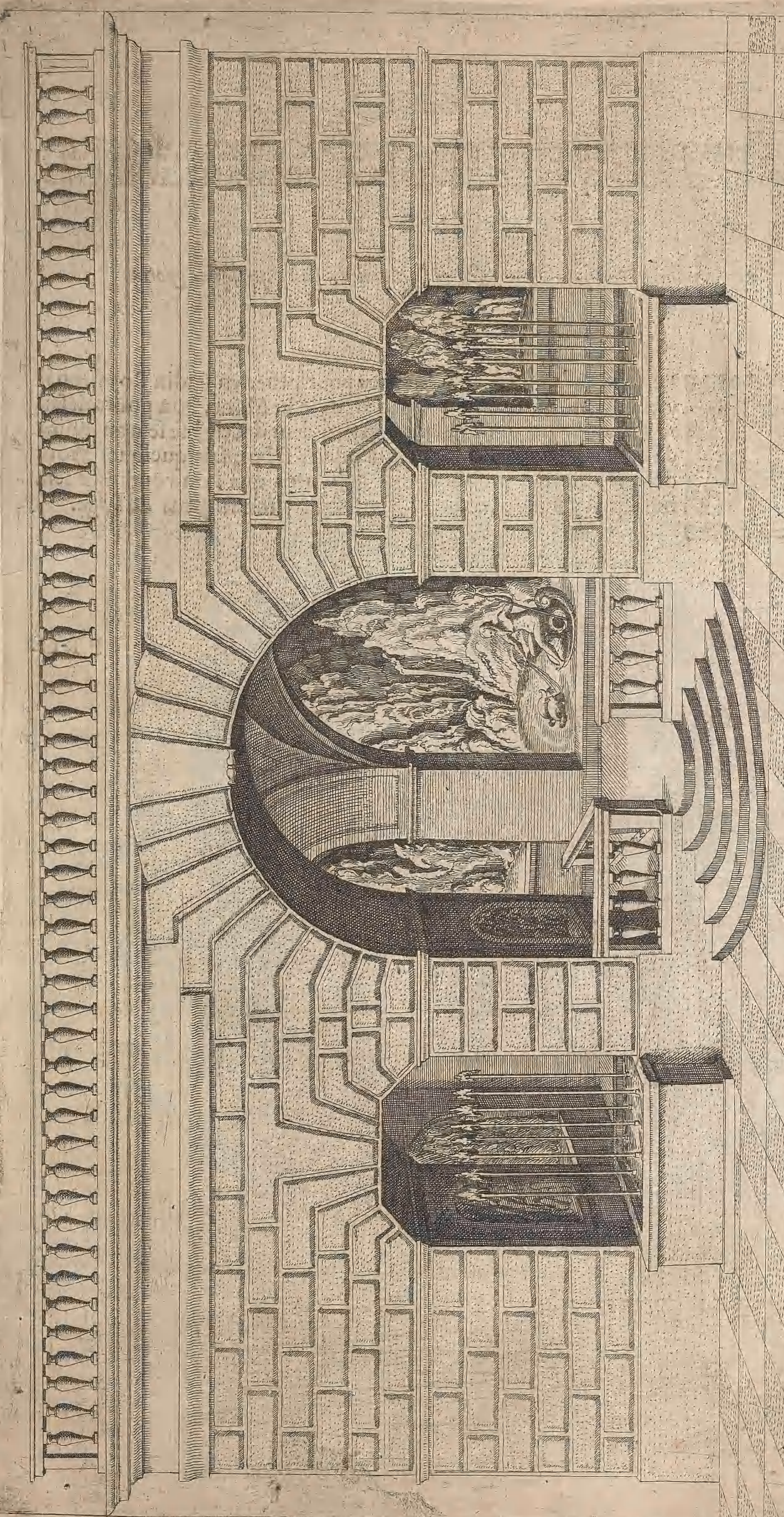




\section{Liure fecond,}

(1) *

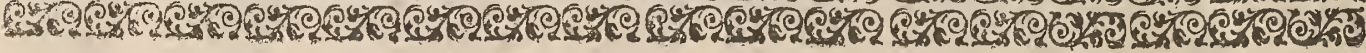

PROBLESME XIII.

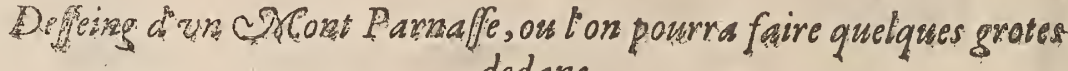

dedass.

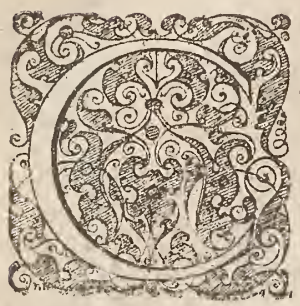
re quelques grotes artificielles, la grandeur d'iceluy fe fera au moins de octante piẹds par dehors en diametre fi l'on fait quelques grote dedans, finon il fe pourra faire auffi petit que l'on voudra \& fera bon qqu'il y aye de l'eas à l' énviron de virons uz. pieds de large. 


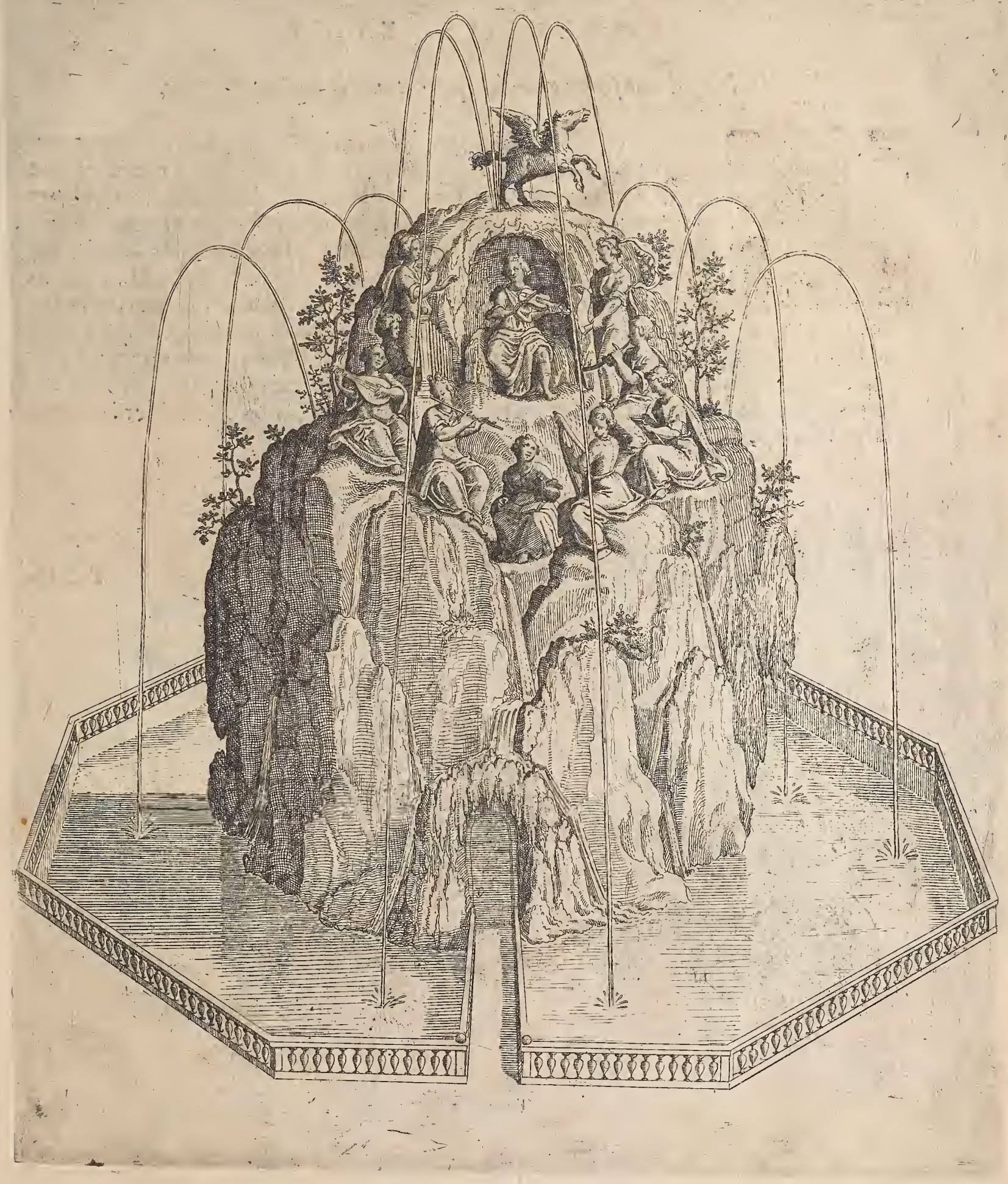




\section{Liure fecond,}

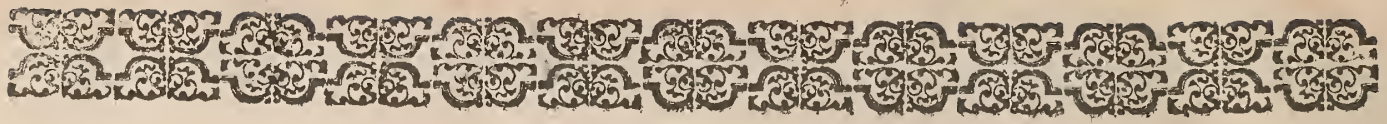

\section{P R O B LES M E XIII I}

\section{Deffeing d'vne frgure grande reprefentante le SCont $T$ molus.}

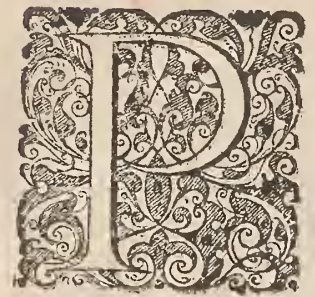

Assa n à Pratelin cinq milles pres de Florence, entre autres ouurages de grotes dont ladice maifon eft richement ornee, ie vis vne figure d'vn grand Ciclope dans, le corps, duquel font quelques grotes fot artificiellement faites, \& fuyuant linvention de ladite figure, i'en reprefenteray icy deux autres, affez à propos, aufli pour faire quelques grotes dedans la grandeur de cefte icy, fera au moins ralle, iurquoy elle ent affife, l'an pourra faire quelques grotes, pour reprefenter quel. que fubier à propos pour ladite figure, à laquelle l'on pourra donnerle nom de mone Tmollus pour fuiure la Fable reciree d'Ovide , du jugement que ledit Tmollus fit, entre Apollon \& Midas, ar faire les grotes de dedansjaccordantes à ce fubier a comme fera recité au Problerme fuiuant. 


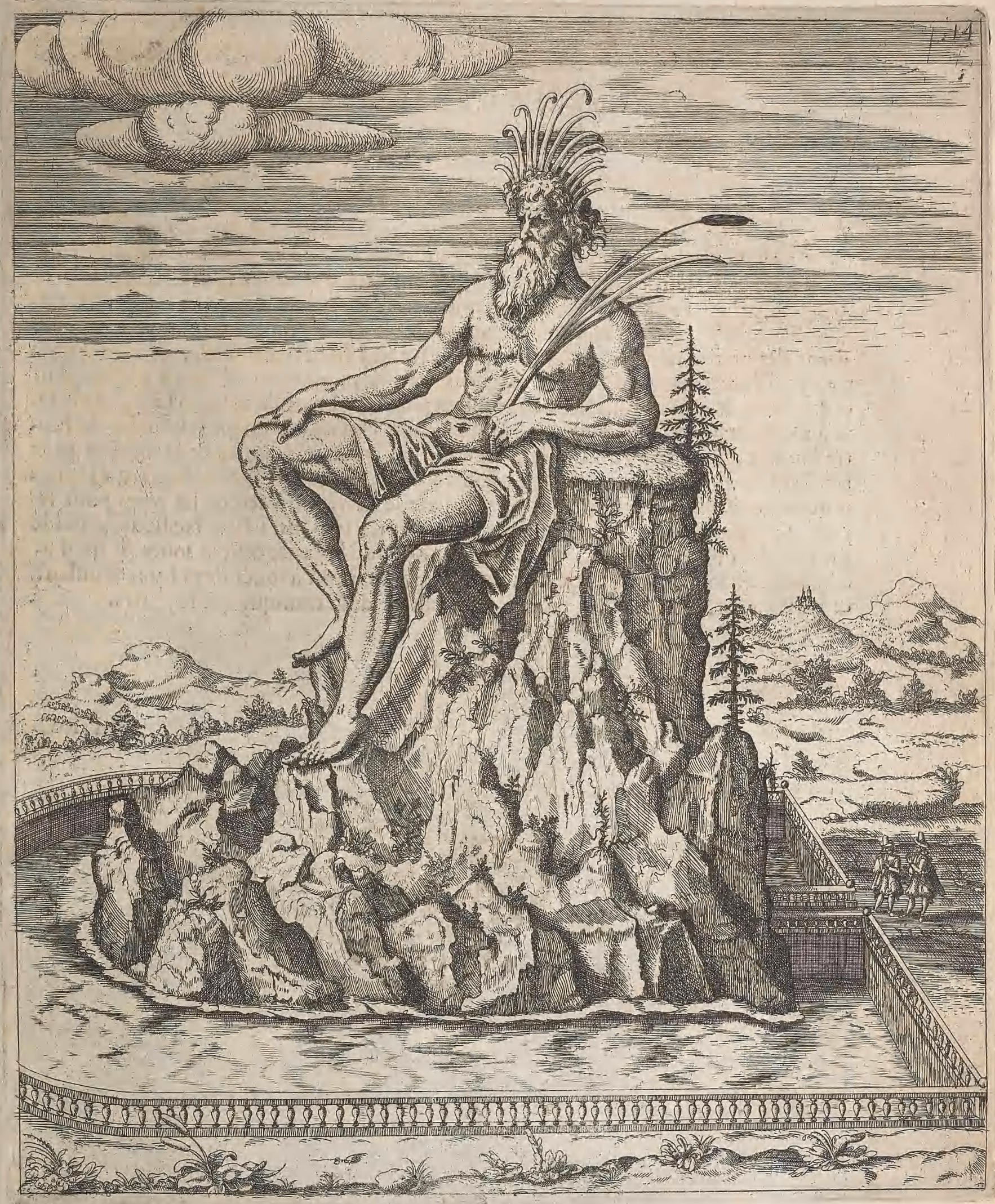




\title{
Liure fecond,
}

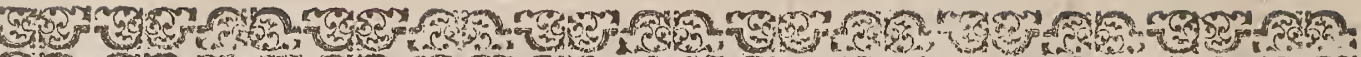

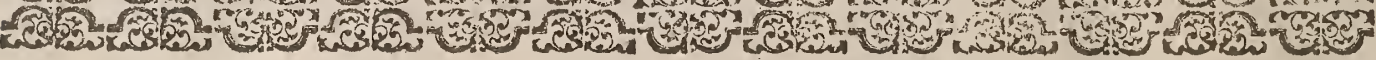

\author{
PRO B L E S E X V.
}

\section{Deffeing de la grate de $\mathcal{T}$ mollus.}

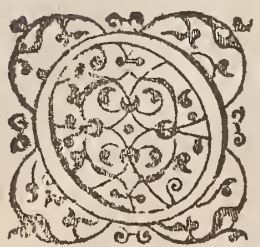

Vide fait recit que Midas oyant le fon du flaiolet du Satire Pan vouloit fouftenir qu'iceluy eftoit plus harmonieux que la Lire d'A pollon, dont ledit apallon fe fentant indigné, voulut faire iuge de ce diferent le mont Tmollus, \& vint comparoiftre deuant luy comme auffi fit Pan, lefquels apres auoir ioué l’vn \& l'aurre, le pris fut donné à Apollon, \& voulant encores Midas fouftenir, par panition luy vindrent des oreilles difne , cefte fable peut eftre fort bien reprefentee, en la grote qui pourroit eftre dedans ledit mont Tmollus, i'en ay mis icy vn deffeing à propos pour ceft effect, \& quand aux machines pour reprefenter la mufique de la Lire, elle fe fera auec deux regiftres de tuyaus d'orgues fçauoir l'vn d'vn trois pieds bouche, \& l'autre fon octaue ouuert, comme fera enfeigné au troifiefme liure, \& la mefme rouë mufiqualle qui fait iouër ladite Lire, fera auffi iouër ledit flaiollet, apliquant de longs porteuents, depuis le fommier iufques derriere le Satyre, où feront les pipes pour reprefenter ledit flaiolet, le mouuement des figures fe pourra faire facillement par le moyen de la rouẹ mufiqualle, \& faut, que quand le Satire ceffe à iouer $\&$ qu'il abaiffe fon flaiollet, que l'A pollon commence auffi toft à iouër de fa Lire, hauffane \& baiffant l'archet de la lire, fuiuant les mefures de la mufique qui fe jouera. 


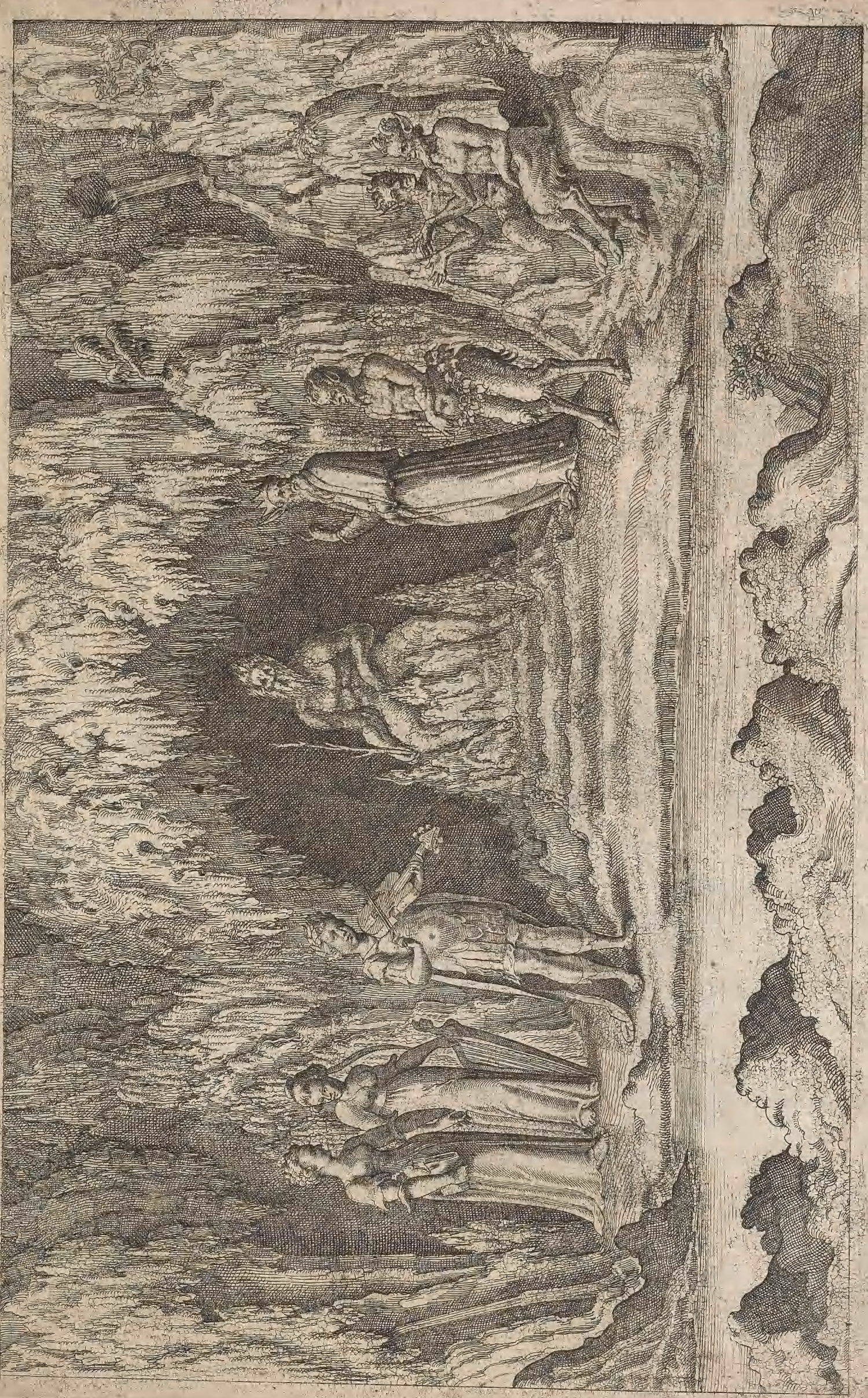




\section{Liure fecond,}

б. 万丂

$$
\text { PRO B.LE S E X X V I. }
$$

Autre deffeing d'one grande figure ruftique pour reprefenter on Flewue, E dedans le corps dicelle le pourra faire

quelques grotes.

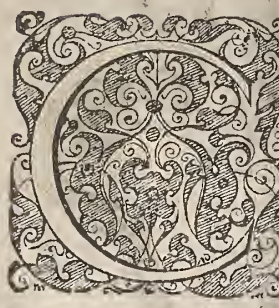

EST autre grande figure fe pourra faire de pierres rúftiques propre pour reprefenter quelque Fleuue, laquelle fera fort propre pour faire quelques grotes dedans, \& $f i$ lon a grande quantité d'eau il fera bon de la faire paffer dans vne grande cruche, que ladite figure tiendra entre fes bras.

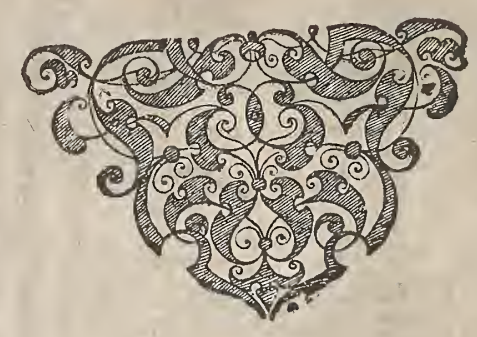




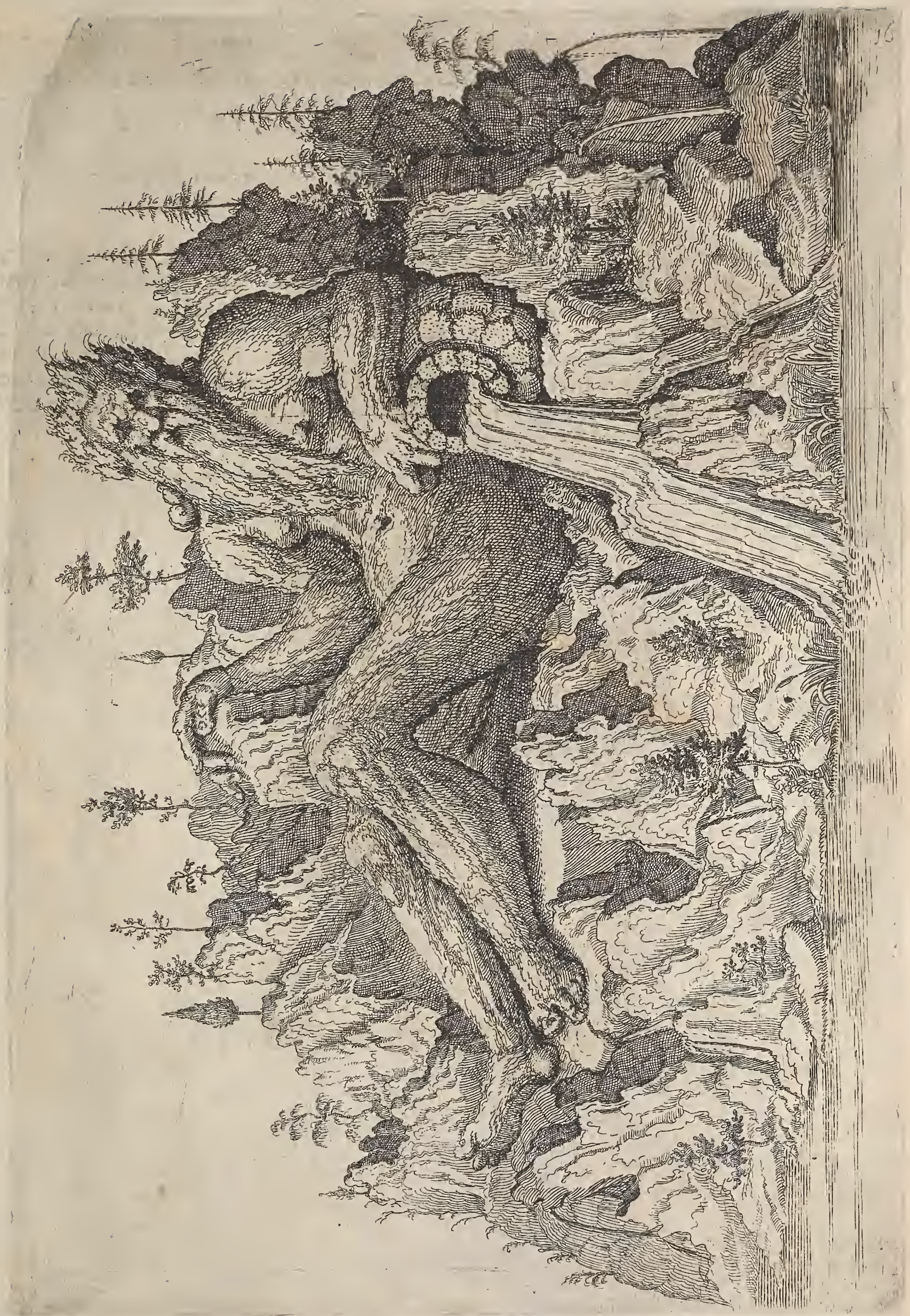




\section{Liure fecond,}

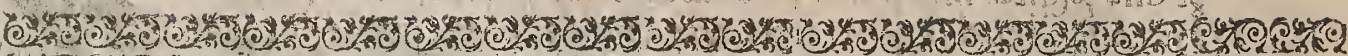

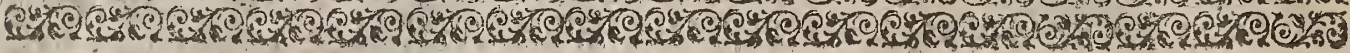

\section{PR OB LES ME X XI}

Deffing diane zrole dorfee qui fo pourra faire dans ba fagure precedente.

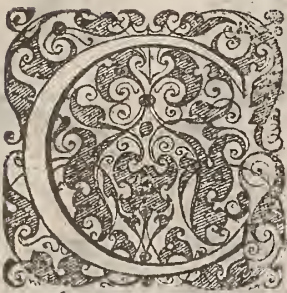

Esta fable d'Orfec, vient encores fort à propos, pour vne grote, laquelle fe pourra faire dans la grande figure precedente, \& 8 le mouvement do la mufique, fe fera derriere la figure, en fortequ'ilfemble, que fe foit elle qui ioüe, \& le mouuement du bras fe pourra faire par une manivelle, qui fera à une des rouès dentelesqui pour. ra cirer, \& lafcher un fil de cuiure attaché audit bras, la méfure \& gné au traifiefne Liure. ordredes tuyaux d'Orgues, pour seprefentex ladite Lire fera enfei- 


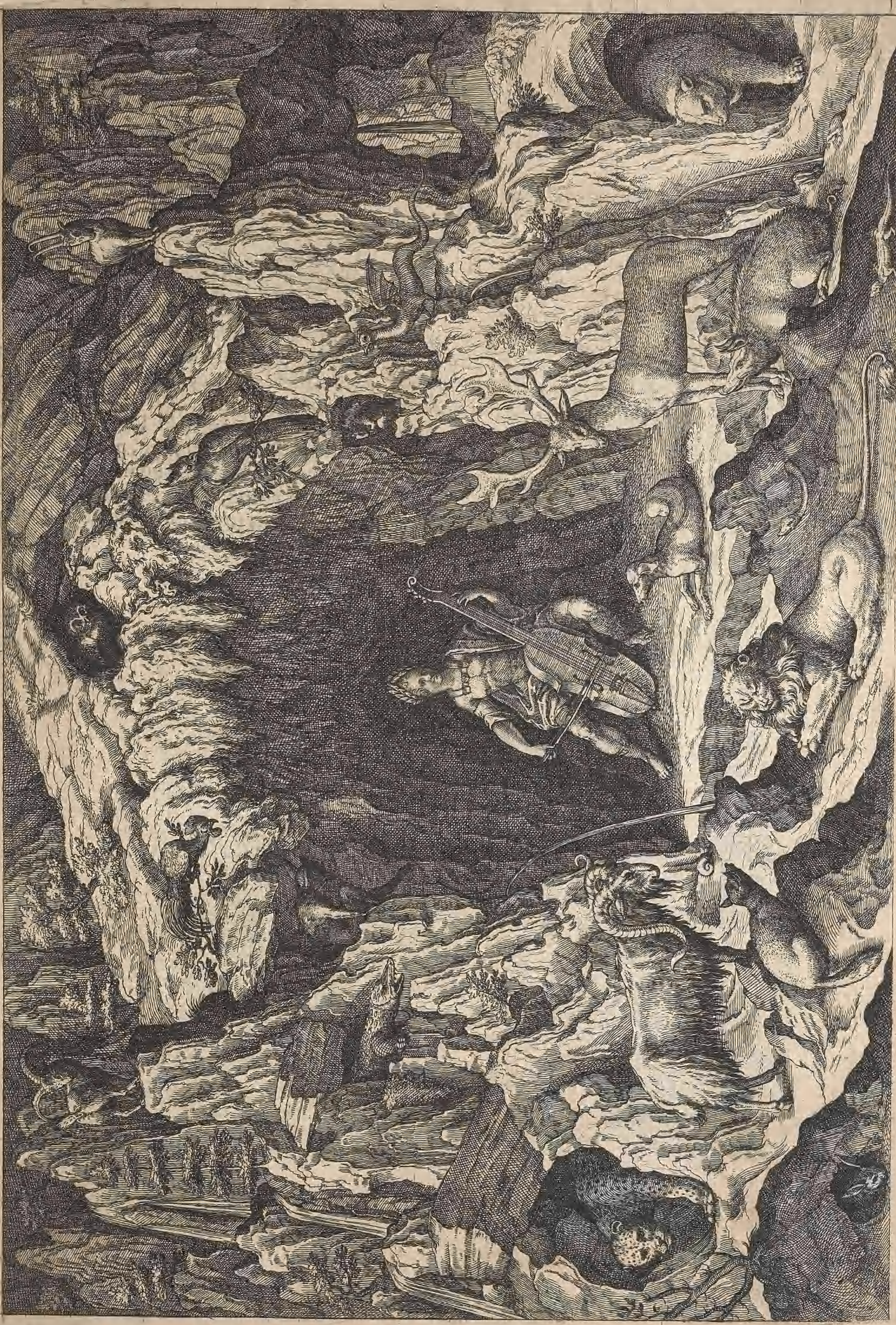




\section{Liure fecond,}

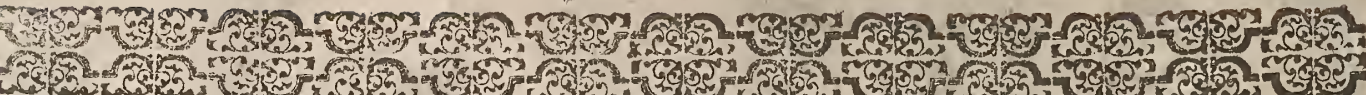

PROBLES M E XVIII.

Deffeng d'une Nimphe qui ioure des Orgueszà laquelle on Efcho respond.

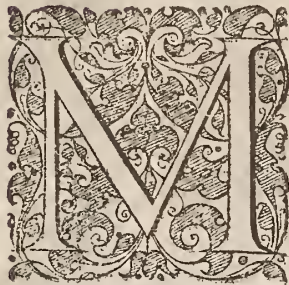

A is fi lon defire faire une grote accomplie divn grand concere de diverfité de regiftres d'orgues, joüant par le moyen de l'eau, l'on pourra reprefenter comme fi une Nimphe joùorr deffus un clavier faus, \& aux cadences qui feront faites en la mufique, ton pourra faire qu'il y aura une autre Nimphe eflongnee dans un creux de Rocher d'ou viendra une relation defdites cadences, ce qui pourracfast par des portevents depuis le fommier, où font les tuyaur de ladite NImphe iufques au lieu d'ou lon voudra faire venir ledir Efcho , \& pour ceft effect il faut auoir huit où dix touches en particulier fur le clavier, pourfairefonner ledir Efcho à propos quand befoing fera, \& faut auffi prendre guarde queles tuyaux qui ferventaudit $t$ fcho ne fonnent fi haut que les autres, à celle fin dimiter mieux la nature, ce qui fera aifé à faire, faifant la bouchę derdits tuyaux un peu plus ętroitea \& leur donnant moins de vent. 


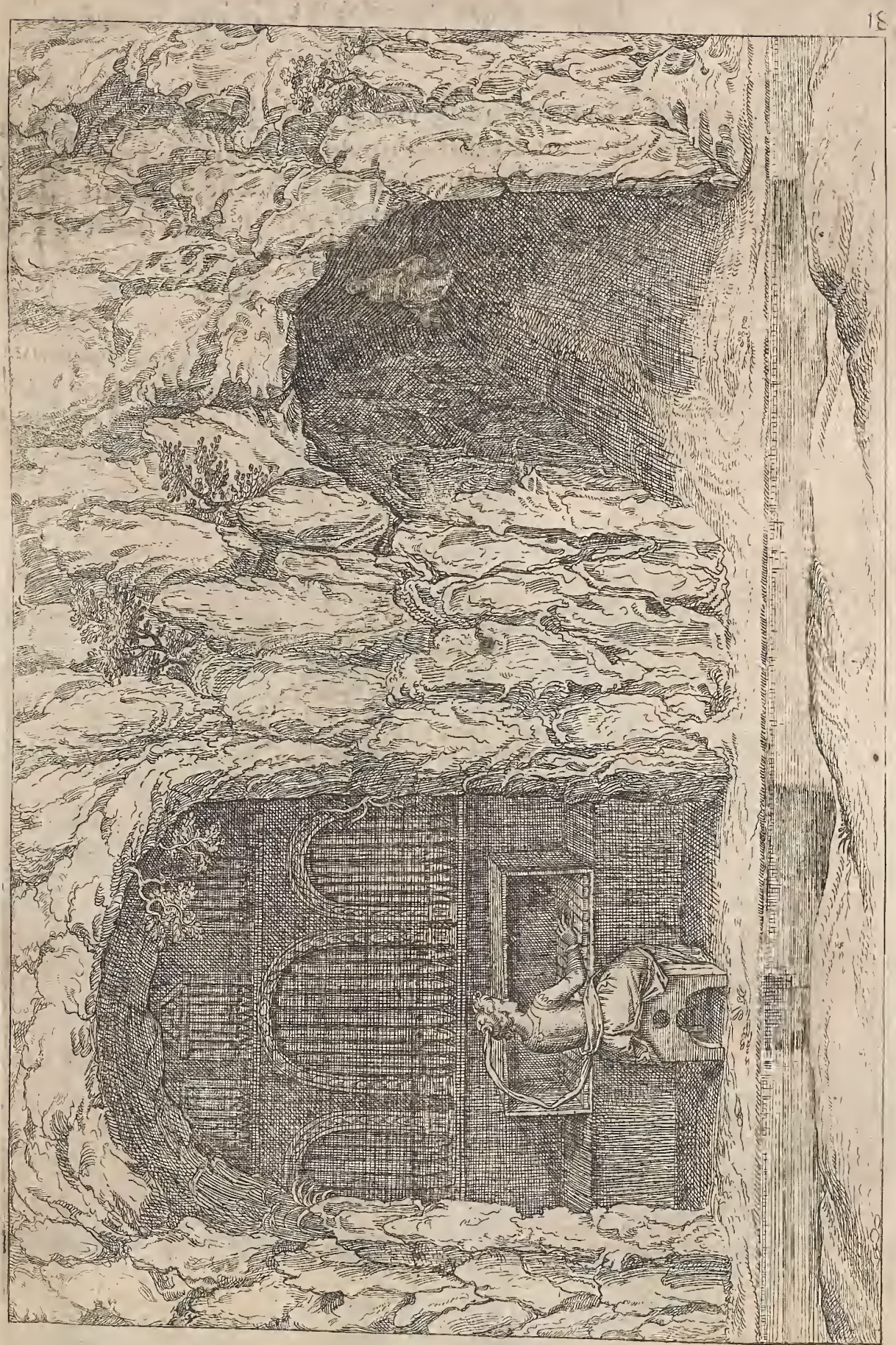




\section{Liure fecond,}

(2)

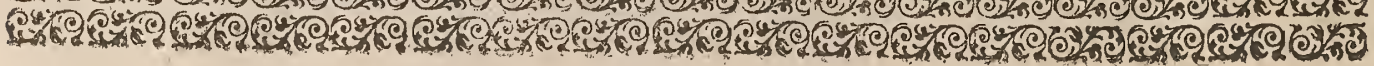

P R Q B L E S M E XIX,

Doreing done Fontaine, propre poun mestre en in lardin.

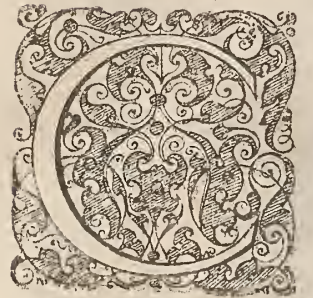

EsTe fontaine, viendra fort à propos, pour mettre dans vn lap din, où 1 l y auroit quantité deau, \& l'eau, qui tombe en forme de cafcade, au long de la Roche denhaut, donnera plaifir à la veuë, laquelle defcendra par va des Pilątręs , pour remonter \& forcir en la Roche d'en bas.

PRO. 


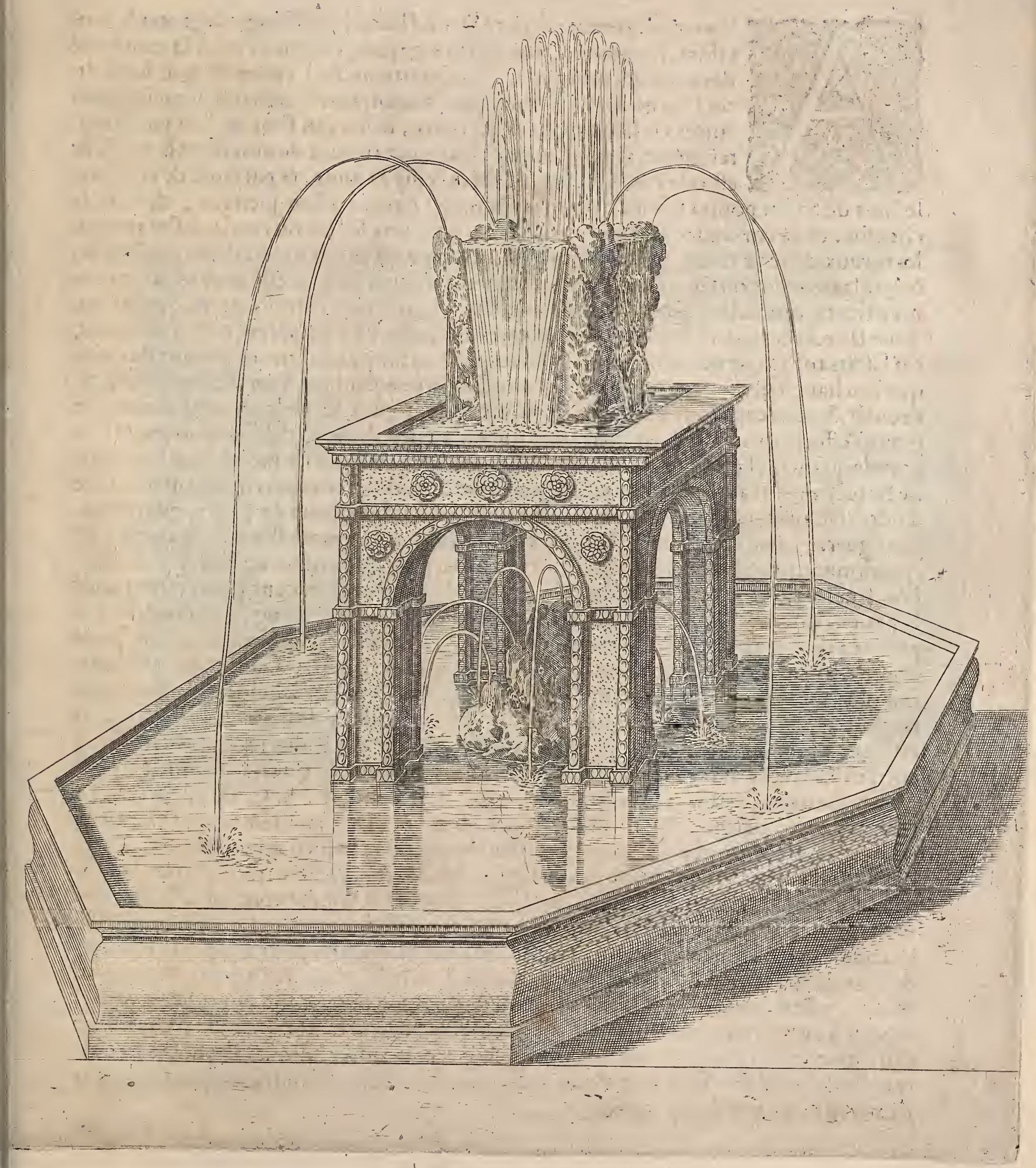




\section{Liure fecond,}

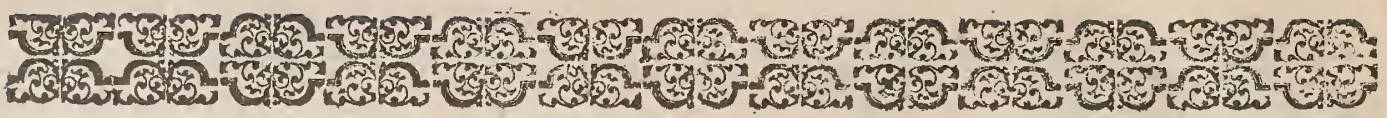

\section{P R O B L E S M E 'XX.}

\section{Pour la conduite des caux de Fontaines.}

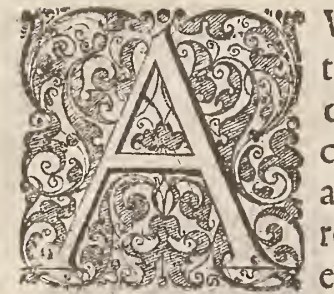

VANT que mettre finà ce fecondliure de deffeings de grotes \& fontaines, i'ay trouué bon de faire ce petit difcours, pour la conduite des eaux des fontaines. Premierement faut entendre que les fources font de diverfes fituations, aucunes en lieux bas \& marcfagcux, autres en lieux hauts \& pierreux, celles qui font en lieu bas \& $\mathrm{ma}$. refcageux, fe pourront conduire par tuyaux debois dontle meilleur eft celuy de Chefne, \& apres celuy D'aune, \& parfautede cesdeux, le bois de Sapin pourra fervir, mais fi la fource eft haure en lieu pierreux, \& dont le chemin, de la conduite foit toufrours de feendant, vers le lieu ou l'on la defire mener, les tuyaux de terre recuite, pourront fervir, pourveu qu'ils foient bien joints enfemble, \& que la trenchee, ou feront posés lefdits tuyaux foicbien ferme, \& de terre folide, autrementlestuyaux debois feront encores meilleurs, \& aufi faut prendre garde que la conduite faite, auectuyaux de pierrecuite, ne remonte point en haut apres quelleàdefcendu, car lefdits tuyaux ne peuuent foufrir la force quel'eau fait, quand en defcendant de quel. que lieu haut (encores que ce ne fut que fix pieds, perpendiculaire)lon la contraint de remonter, \& pourceft effeet les tuy aux de plomb font propres, lefquels peuuent endurer de grands efforts, quand ils font bien fairs, quand au nivellement defdites fources, s'il ya grande quantite, l'on pourradonner furcent pieds, vn pied pourle moins, \& fil' on donne beaucoup d'avantage, il ne fera que mejlleur, \& auffi les conduits n'auront que faire d'eftre fi grands, carl' eau paffe bien plus iufte, ayant beaucoup de pante, que n'en ayant gueres, mais fi le lieu de la fource eitoit fort bas, comme il arrive fouuant, \& que donnant vn pied de pante fur cent, l'eau ne pourroit arriuer au lieu defiré, alors l'on fera les tuyaux de la conduite fort grands, \& dem i pied furcent, pourra fervir pour la pante, il fera auffi fort neceffaire quand la fource vient de loing, de faire des receptacles à cinq cent pas, ou à mille pas au plus, livn de laucre, lefquels ferviront pour donner aïr audies conduits, \& auffi sily avoir quelque defaur à la conduire, il $\mathrm{fe}$ pourroit plus aifement trouuer la faute \& la reparer, il arrive aufi quelquefois que leau ne peut avoirfon cours, faute des vents, ce qui fait penferà plufieurs, que le conduit eft bouché , mais ceft accident arrive, faute de n'avoir mis lefdits recepracles, enlieux convenables, \& davoirmal affis les tuyaux de la conduire, ce queiedemonftreray icy, par vn exemple, foir la fource marquee A. \& le conduit B. C. D. F. allant vn peu en pante, aulieu B.C. \& defcendant fort en D. puis reçmontantvin peu en E. mais non fi haut que C. \&en plufieurs endroits, l'on eft contraint de farre la conduite de cefte façon, à caufe des boffes, \& fondrieres, quelon trouue fur lechic min de la conduite, \& ainfi s'ily a quelque chore à reparer, à la dicte condurte, \& queton defire vuiderl'eau des tuyaux, elle nepourra fortir de la fondnere $D$. pour eftre plus bas que E. tellement quel'eau reftante, quandl'on viendra pour remplirle conduit de l'eau de la fource, ladite eau ne pourra paffer outre, àcaufe de l'air qui eft entre $B$. \& C, tellement que le conduit demeurera ainfi, fans avoir fon cours, \& pour remedier à cecy, il faudra faire vn efvent ou receptacle au lieu C. à celle fin que l'arr forte du conduit, \& que l'eau fe mette en fa place, \& alorsl'eau aura fon cours, comme elle doibt, il fera bon aufi de faire des efvents au bas des fondrieres, pour netcoyer les tuyaux, quand il fera befoing. 


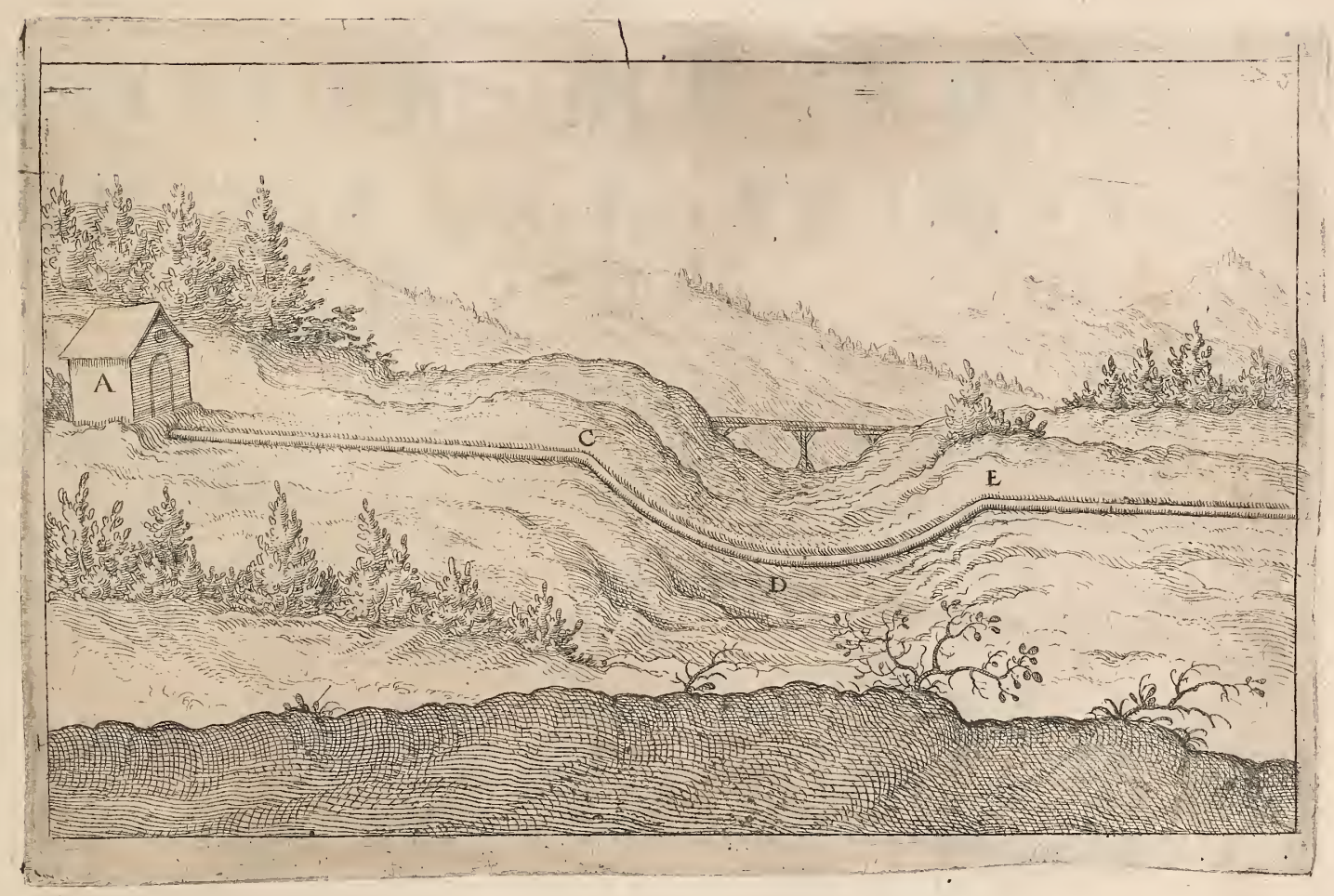



L I U R E

\section{T R O IS IES ME TRA I- T A N T D L A F A R I Q V E DES OR G VES.}

$P \backsim R$

$S A L O M O \mathcal{X} D E$ CAUS $7 N G E N I E V R$ ET Architecte de fon Alteffe Palatine Electoralle.

A Francfort en la boutique de lean Norton 16 I 50 


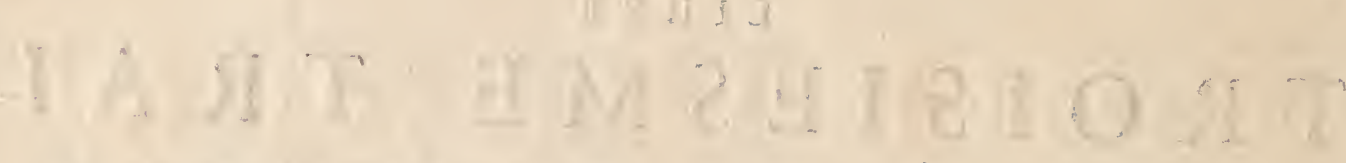

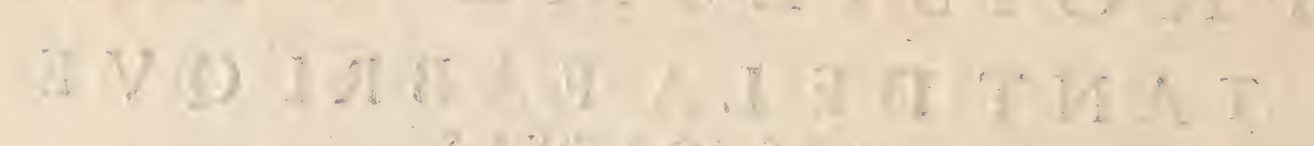

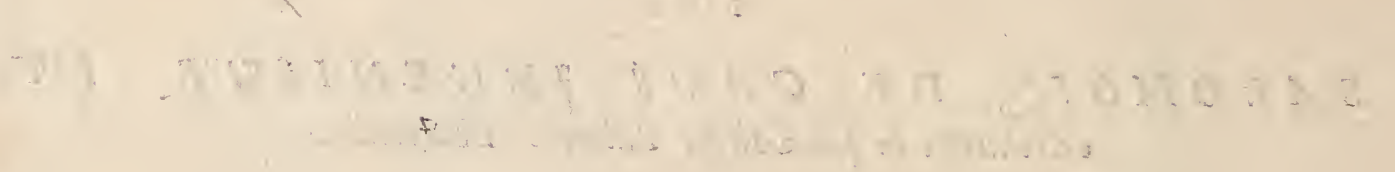




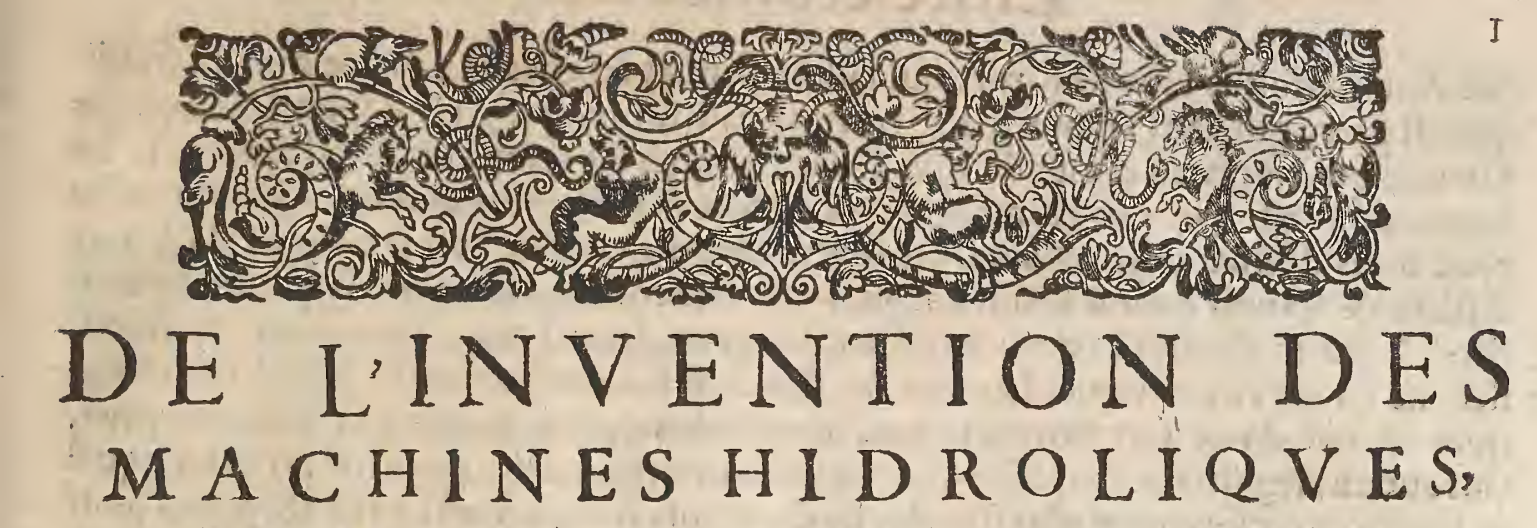

ET ORGVES, ET DE LACROISEMENT

- QVI I A ESTE' FAIT DEPVIS.

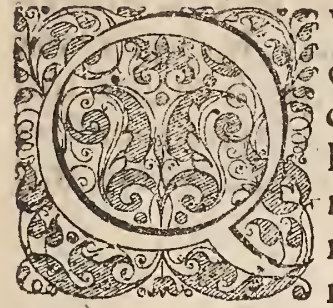

V AND à l'inuention de l'inftrument mufical vulgairement apelé orgue il eft fort difficille d'en trouuer linuenteur, premieremene à caufe que ledit inftrument n'a aucu particulier nom, comme à vn Lut, vn Cithre, vne Guiterne, ou autres tels inftruments, car ce mot organo, eft grec qui fignifie inftrument, qui eft vn nom gene. ral, pour routes chofes que ce foit par le moyen de laquelle, aucune autre chofe eft faite; comme vn marteau, vne fie, vn couteau, \& autres chofes femblables, font organes auec lefquels vn ouurage eft mis en perfection, auffi font toutes fortes dinftruments muficaus dits organes, \& l'ouure qui doibt eftre faict par iceux eft la mufique, \& ainfi fi quelque ancien aucheur parle de l'inuention des orgues, s'il ne pecifie la façon dudit inftrument, loon ne pourra pas iuger que ce foic celuy duquel nous vfons à prefent, fecondement à caufe que linuention d'aucune chofe que ce fort (\& fpecialement d'vn art difficile \& qui defpend de plufieurs autres) commence auec vne fi grande fimplicité quion n'en remar- que pas l'Autheur, \& quelque fois plufieurs années voire par centaines fe pafferontatuanc qu' on aye ataint la perfection d'vn art commencé, quand aux autheurs qui one parlé dédites orgues, le plus ancien qui nous eft cogneu eft Herone Alexandrin lequel au $75 . \& 76$. problefme de fon liure de firitalibus monftre à l'vn la fabrique d'v. ne machine hidraulique, \& à l'autre la fabrique d'vne organe, dont les pipes fonnent auec le vent, apres luy, Vitrune fait vne defcription d'v ne machine hidraulique. Or viruneliure que ces Orgues \& machines hidrauliques fufent telles que les noftres de maintenant il femble y auoir grande difference, veu qu'en la defcription des antiques il n'eft parlé diaucune rouë mufiqualle, par laquelle fe pourroit fonner vne chanfon à plufieurs parties, ny de beaucoup d'autres parties neceffaires pour la perfection derdites machines, \&y a quelque aparence que lefdites machines ont efté faites pour fonner auec la main, \& les antiques n'ayant encores trouué linuention des foufflets, pour les $y$ adioindre comme nous faifons à prefent, vfoyent de vaiffeaux, lefquels fe remplifaris deau caufoit lair d’en fortir, lequel faifoit fonnerles pipes, comment il fe peut comprendre en plufieurs theorefmes dudit liure de Herone, \& auffi au neufuiefme liure chapitre neufuiefme de Vitrune ou il dit que Stefibie qui viuoit vn peu auparauant, ledit Herone trouua beaucoup dinuentions pour reprefenter la vois d'oifiaux, \& au. tres fubtilitez, par le moyen de l'eau, \& auffi ledit Vitrune parlant de la machine hıdraulique met en auant lufage de deux pilons feruans à donner le vent aux tuyaux \& \& en oultre dit que les marches, ou touches du clauier, doiuent eftre preffez par les toigts de l'Organifte , ce qui demonltre quil faloit fe lesuir des doigts pour iouer fur le clavier, \& que lefdites machines fe nommoyent hidrauliques teulement à aufe 


\section{Liure troifiefme,}

que l'eau caufoit le vent de fortir, qui faifoit fonner les tuyaux, car ce mot hidraulique eft grec, qui vaut autant à dire comme eau ronnante. Or depuis le temps de Vitrune qui viuoit au temps de Iıle Céar, iurques au temps du Roy François I. les fciences ont efté forc peu eftimées, \& y a eu fort peu dihommes doct es qui ont vefcu pour nous donner cognoiffance des inuenteurs des chofes, ceft pourquoy il eft fort difficile de fçauoir quànd lefdites orgues ont commencé a eftre en vfage auec les fou* Zastin. fup flets, * Zarlin dit auoir eu vn fommier d'orgues, lequel auoit ferui dans vn mona-

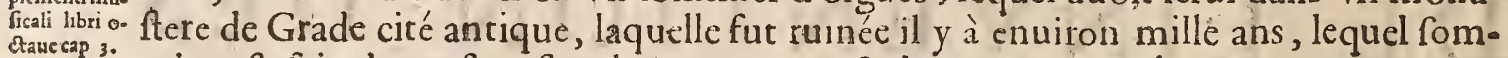
ctauc cap 3. mier eft fait devne fort fimple taçon, auec feulement is. touches \& trente tuyaux, fans aucuns regiftres, iecroy bien que ce fommier a efté vin des premiers, d'autans qu'il ne pourroit prefque eftre plus fimple, \& du depuis l'on à aioufté tant de pieces pour la perfection defdites orgues, que à prefent elles furpaffent toutes fortes d'inftruments en douceur \& harmonie auffi c'eft celle qui reprefente le mieux la voix naturelle de tous les aurres, \& y agrande proximité entres vne orgues bien ordonnee, pour reprefenter les voix humaines, \& les voix naturelles, aufi les Organes qui caufent le fon des orgues, fe peuuent fort bien comparer aux Organes, qui caufent les voix humaines, les foufflets aux poulmons de l'homme, les foupapes, aux léures, le clauier aux dents, les tuyaux à la gorge, la main qui iouë a la langue, en forte que fi chacun ton audites, orgues, eftoit diuifé en $9 . \&$ Io. partic, comme ie pretends cy apres monftrer à faire ladite diuifion, mefmes les voix, quelques bonnes qu'elles fuffent \&-bien maniees, ne pourroyent furpaffer ledit inftrument.

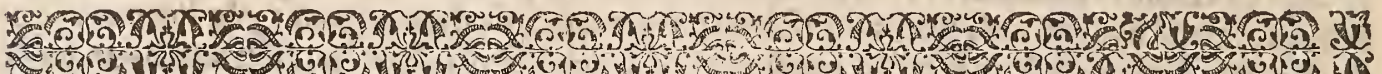

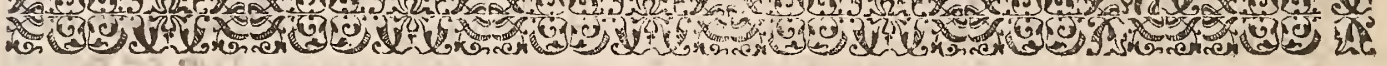

\section{Ce qui eft requis pour la fabrique des Orgues.}

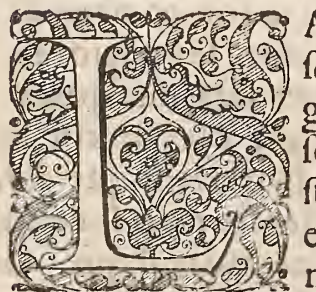

A fcience de bien faire \& ordonner vn ieu d'Orgues, eft laboricu. fe, plaine de grande induftrie, \& requiert vn homme qui aye la co. gnoiffance, au moins de trois arts, premierement eft befoing qu'il foit bon muficien, tane en la theorique, pour bien ordonner la mefure conuenable aux tuyaux, comme auffi en la pratique, pourioüer \& bien accorder lefdits tuyaux, les vns auec les autres, fecondement faut qu'il f̧̧ache l'art de plomberie, pour bien fçauoir ietter le plomb \& leftain en table, \& fabriquer les tuyaux, chacun en fa proportion, tiercement eft auffi neceffaire, qu'il aye bon ne cognoiflance de l'are de menuifferie, pour fçauoir bien ordoner ce qui defpend du fommier, des regiftres, \& foufflets, \& ayant bonne cognoiffance de ces trois arts, il fera capable deftre bon maiftre, \& dautant que ie ne n'ay veu encores aucun autheur, qui aye donné intelligence de ceft art, il m'a femblé bon \& neceffaire pour l'accompliffement de ce liure, de demonftrer ce qui defpend de ladite fcience, tant pour feruir a aucunes machines hidrauliques traitees en cedit liure, comme auffi en quelques autres conftructions d'Orgues, ie commen. ceray doncques à monftrer les mefures propres \& conuenables. pour les tuyaux puis apres toutes les pieces conuenables \& defpendantes de ladite fcience. 


\title{
Traitant de la Fabrique des Orgues. ,
}

\author{
P R O B L E S M E I.
}

\section{La maniere comme il faut jecter le plomb Es leftain pour lo fabrique des Orgues.}

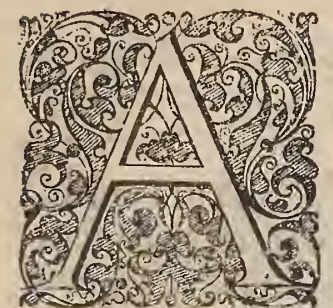

VANT que de parler de la mefure des Orques, ie monftreray icy la façon d'aprefter le plomb \& l'eftain, pour la fabrique des tuyaux, doncques l'on prendra du plomb le plus doux que l'on pourra trouuer de fort viel, il ne fera que meilleur, prenant garde qu'il n'y aye aucune foudure auec en le fondant, puis l'on aura vne table de pierre, ou de bois bien vnie de Iz. où Is. pieds de long, \& vn \& demi où deux de large, laquelle fera plus haute efleuée d'vn cofté que de lautre, comme la figure le demonftre, \& felon l'efpeffeur quel'on defire donner au plomb, car le voulant faire delié, il faudra qu'elle panche fort, \& faudra doubler ladite table par deffus de 3 . ou 4 . doubles de bonbazin où de couftil, acommodé auec de la craye, pour le rendre plus vni, l'on aura auffi vne caffetre, nommée rabor marqué B. laquelle fe pourra gliffer au long de ladite table, en forte que le plomb eftarit fondu de bonne forte ce que lon cognoiftra en pouffant vne pécite piece de pa. pier dedans, \& le retirant viftement, sil fe brufle, ledit plomp fera trop chaud, mais fi la coulear du papier change, \& quil deuiene fort roux, il fera affez, puis en faudra ietter fur le bout de la table, laquelle à caufe de la pente quelíe a, \& le rabot eftant fait en forte, comme il fe peut voir en la figure, le plomb demeúrera enclos, entre lestrois coftées dudit rabot, \& incontinent celuy qui tiendra ledit rabot, le gliffera au long de ladite table, felon lefpeffeur qu'on y veut donner car en tirant fort vifte, il fe fera fort deflié, \& doucement il fe fait plus épais, \& faut garder de ne le tirer par fauts car lefpeffeur ne feroit efgale, \& quand à l'eftain il fe ietrera auffi de la mefme façon, mais il ne faut pas qu'il foit fondu fi chaud, \& faut aufi prendre garde que fi ceft d'eftain d'Angleterre trefpur, de mefler cinq ou fix liures de plomb auec vn cent dudit eftain lequel le fera coufler mieux en iettant.

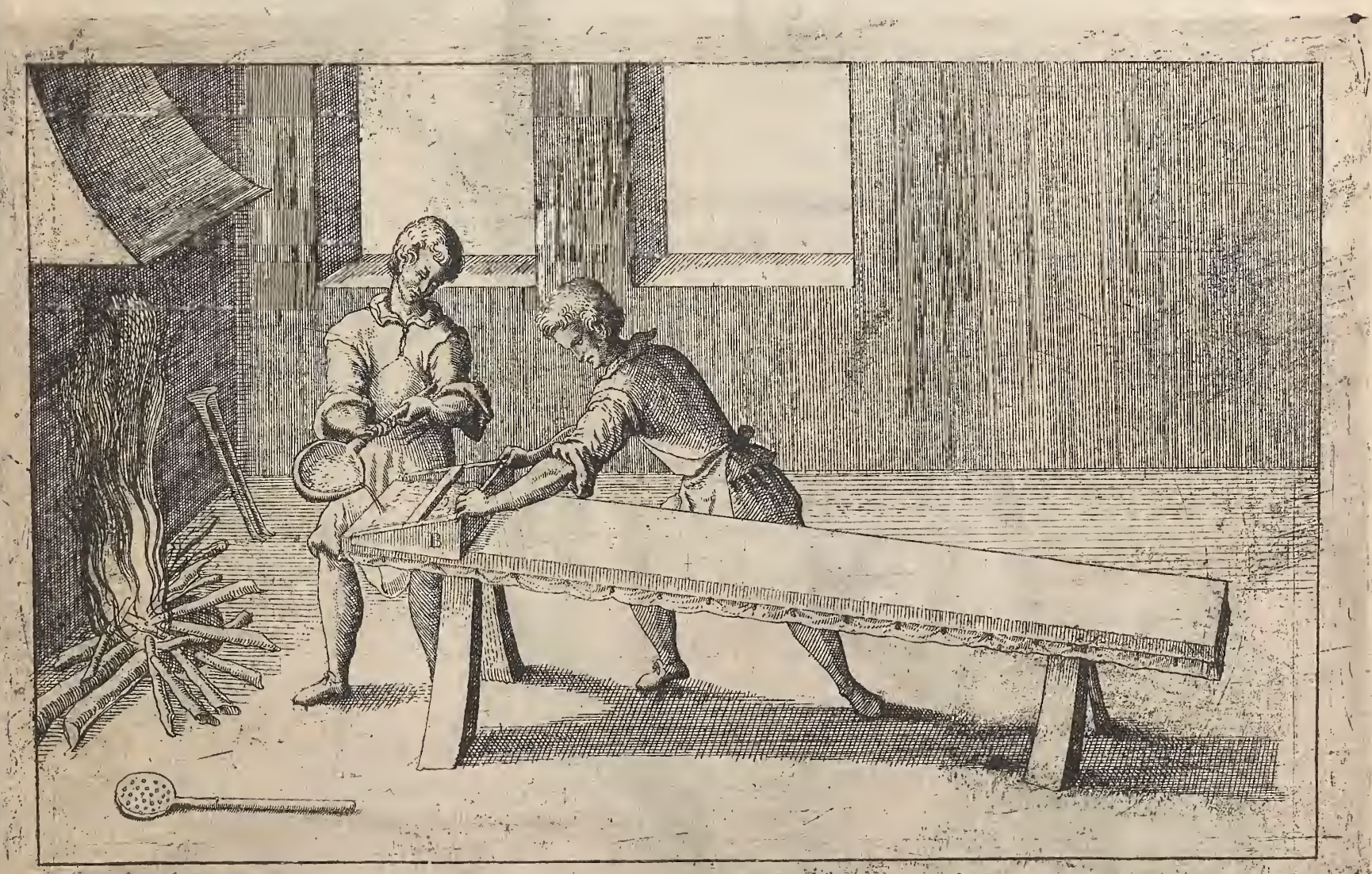




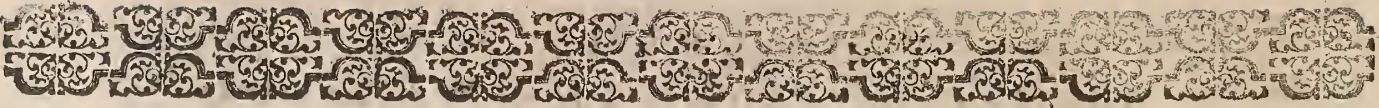

P R O B L E S M E II.

\section{Inftrument par leguiel on fera le plomb ES eftain fort ony Ee d'rine clgale efpeffeur.}

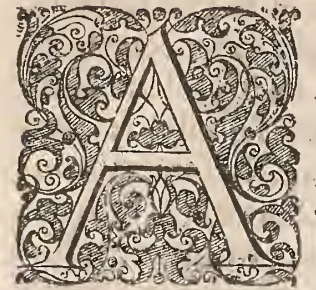

PrES que le plomb \& l'eftain fera jetté en table, lon aura vn inftrument pour le faire vny, fait comme il fe peut voir en la fuiuan. te figure, ou il y aura deux rouleaux de fer ou de cuiure marquees A. B. bien ronds \& vnis de tous coftez \& a laxe de celuy A il y aura vne croifée pour tourner ledit rouleau a force de bras, \& entre lefdits rouleaux l'on mettra la piece de plomb que lon defire faire vnie, \& tournant ladite croifee, le plomb paffera entrelefdits rouleaux, \& fe fera fort vny \& liffé, '\& à celle fin de donner telle efpeffeur au plomb que lon voudra, les deux vis marquees C. $D$. fe tourneront $\&$ poufferont vne piece de cuiure contre laquelle laxe de rouleau, de haut tourne ce qui fe pourra fort bien comprendre par la piece particuliere marquee E. \& tout ainfi comme lon vie du plomb, l'on vera auffi de l'eftain.

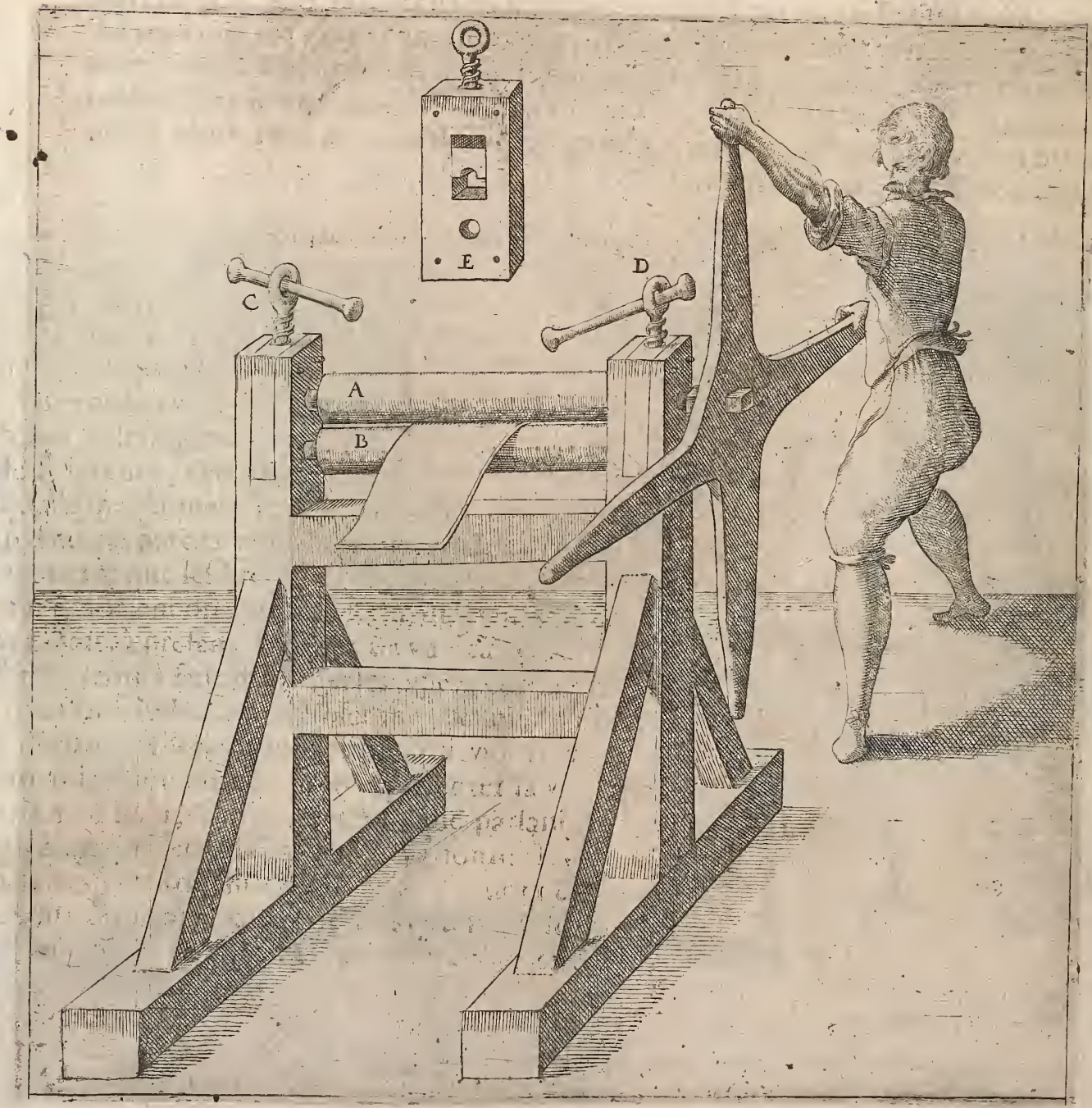




\section{Liure troifiefme,}

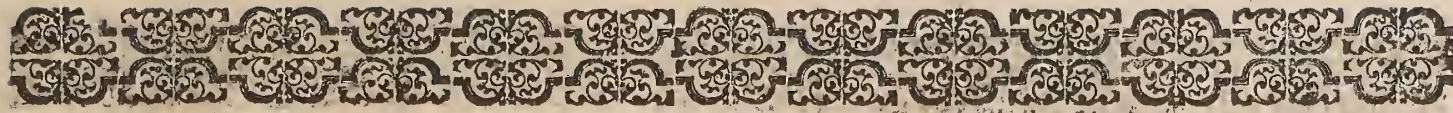

P R O B L E S E III.

\section{Comme il faut donner la mefure au Siftefme communement dit Diapajon.}

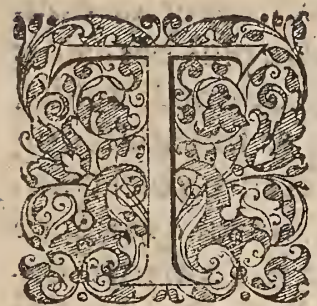

Ortes les Orgues. bien ordonnées font faites en forte que les tiay: aux quifonnent F. FA VT. font de 3. pieds en longueur, ou de fir ou de douze, ou de pied \& demi, la raifon eft, à celle fin d'accom. moder les voix auec lefdits tuyaux, car sils n'auoyent cefte longueur ou bien pres dicelle, lefdites voix feroyent fort contraintes c'eft a dire trop hautes ou trop baffes pour s'accom moder auec, nous commencerons doncques par vn Siftefme d'vn pied \& demi en longueúr marqué F. H. qui fera la longueur du tuyau F. F A V.T. depuis la bouche iafques au bout de haur, apres l'on diuifera toute ladite longueur F. H. en deux parries efgalles au point $f_{0} \&$ ainfi $f_{0} H_{\text {. }}$ fera la longueur du ruyau Diapafon ou octa. ue contre F. H. apres toute la longueur F. H. fera diuifée en trois parties ef

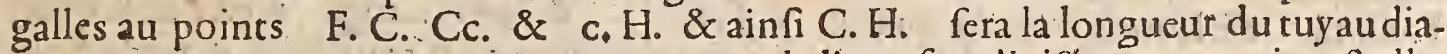
pente ou quinte contre F.H. apres toute la ligne fera diuifée en 4 parties efgalles au points F. B. B.f. f.ff. \& ff. $H_{0}$ \& ainfi B. H. fera diateffaron, ou quate contre F. H. apres toute la ligne fera diuifée en 5 parties efgalles aux points F. A. A. D. D a. a. a a. \& a a. H.. \& ainfi A. H. rera diton contre F.H. apres foit toute la ligne diuifée en 9. efgalles parties \& ainfi G. H. qui contient huit defdites parties fera vn ton maior, plus haut que F. H apres foit la partie D.H. diuifée en 9. parties efgalles, \& E. H. qui contient huit derdites parties fera vn ton maior plus haut que $D . H$. \& apres Ion diuifera la partie $A . H$. en 9 . efgalles parties \& $H$. qui contient huit defdites parties fera vn ton maior plus haut que $A H$. tellement que par cefte diuifion lion aura les longueurs des tuyaux compris foubs le premier Diapafon a fçauoir F. G.A.B. . C.D.E. f. \& auff partie des autres interualles fuperieures, \& pour auoir le refte lón diuifera celles de bas par moirié \& mettant la. dite moirié au deffus de $f$. fe fera toufiours l'octaue de celle de bas \& ponrauoir les interualles au deffus de ff. lon diuifera celles dupremier Diapafon en 4. ou du fecond en $2 . \&$ ainfi lon les mettra au deffus de $f f$. refte pourauoir la mefure des feintes, premierement celle entre C. \&D. qui doibt eftre vn diton contre A. c'eft pourquoy diuifant la partie A. H. en 5 . parties efgalles C. H. qui contient 4 defdites parties ce fera la feinte entre C. \&D apres pour auoir la feinte entre D.\& E. I'on diuifera la partie de ligne $\exists \mathrm{H}$. en 5 . efgalles parties \& 4 . d'icelles marquées D. $\times \mathrm{H}$. fera la feinte, apres pour auoir la feinte encre G. \& $\mathrm{A}$. l'on diuifera la partie C. * H. en 3. parties \& adiouftant encores vne defdites parties audits 3. l'on aura la partic G. $\nless \mathrm{H}$. qui eft la feinte entre G.\&A. apres pour auoir la feinte entre

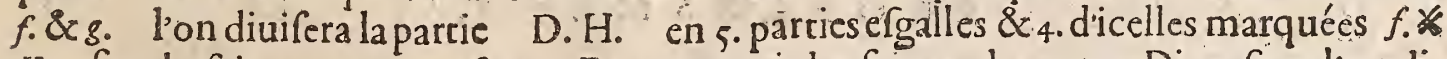
H. fera la feinte entre f. \& g. Er pourauoir les feintes des autres Diapafons l'on diuifera ceux cy en deux pour les metre au deux fiefme \& en quatre pour troifiefme \& ainfi l'on aura les 43. mefures de tuyaux depuis. F. iufques à c c c.

Apres pour auoir les largeurs defdits tuyaux, premierement l'on diuifera la longeur -F: H. en s. parties efgalles \& 2 . d'icelles feront pour la circonference du tuyau $F$. c'eft pourquoy l'on metra ladite ligne de la circonferencè à droit augle fur $F_{0}, \&$ Rera marquée F. N. apres lon tirera fur le point ccc. vne ligne ccc. P. efgallè̀ Ccc. H. \& apres l'on tirera vne ligne-P.N fur laquelle feront tirées toutes les pararelles de tous les points des longueurs \& ainfi toutes lefditeslignes monftreront les circonferences de cous les tuyaux. 


\section{Liure troifiefme,}

PROB L E S M E I V:

\section{Pour donner la mefure aux autres Sifte mes bouschees.}

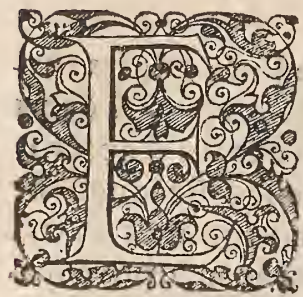

T quand lon voudra faire vn Siftefme, vn Octaue plus bas que le precedent, il faudra que tous les tuyaux foyent iuftement de dous. ble longueur, \& fi on le veut auoir vne quinziefme plus bas, alors il faudra que chafun tuyau foit 4. fois auffi long comme le fufdit, \& fi on le veut vn 22. plus bas, alors.il le faudra 8. fois auffi long me lon y procedera, pour la doubler, il faudra faire vn quarré de la ligne F. N. marqué icy G. F.E. H. apres il faudra prendre le diamettre dudit quarré F. H. lequel feruira pour vn des coftez du quarré. A. B. C. D. \& ainfí les quatre lignes des coftés du quarré A.B.C. D. eftant iointes enfemble fera la circonference du tuyau F. de trois pieds bouché ce qui fe demonftre, dautant que ledit quarré A. B.C.D. eftiuftement doubleà celuy E.F.G.H. car le triangle F. G. H. eft la moirié dudit quarré E. F.G.H. \& ledit triangle, n'eft que le quart du grand quarré, \& fi l'on defire auoir la circonference d'vn regiftre de 6 . pieds bouché, l'on doublera encores ledit quarré A. B. C. D. \& peur la circonference d'vn de iz.pieds, on la quadruplera, \& ainfi iufques à linfini, lon pourra auoir des tuy aux grands ou petits.

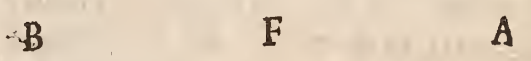

$\mathbf{E}$

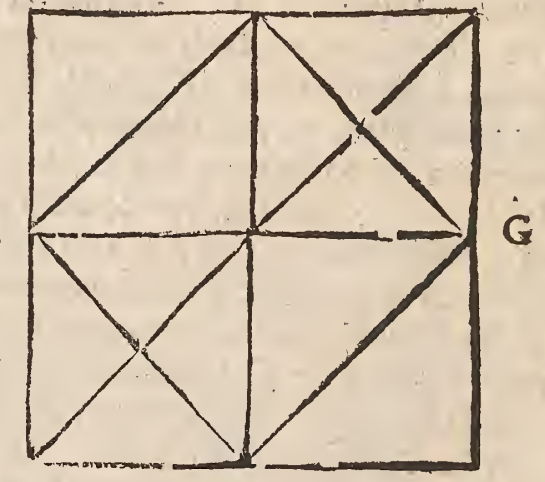

D

$\mathrm{H}$

C

\section{PROBLESME V. \\ Pour faire les Siftefmes de tuy aux ouwerts.}

4ค19:

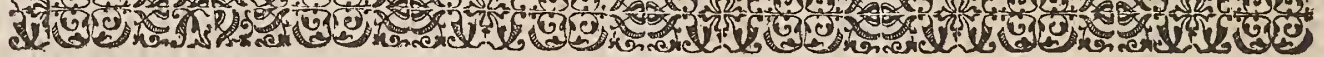

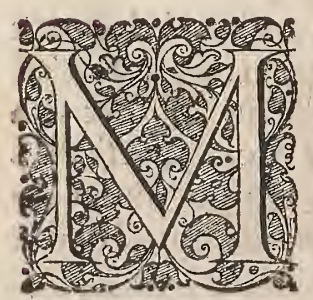

A rs fi lion veut faire des tuyaux ouuerts l'on diuifera iuftement la moitié de la largeur F. N. au point R. \& autant a ccc. P. au point Q. \& ainfi l'on tirera vne ligne $R . P_{x}$ trauerfante toutes les pararelles tellement que cefte ligne donnera toutes les circonferences des tuyaux, \& fi le Sifteme eft plus grand on fera la femblable diuifion. 


\section{Liure troifeerme,}

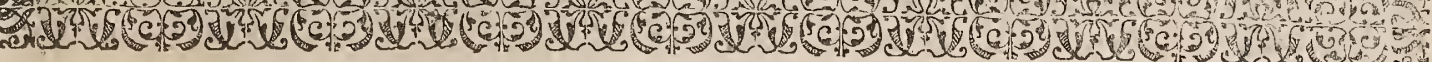
P R O B L E S M E VI.

\section{Pour faire les Sistefmes de tuy aux acheminee.}

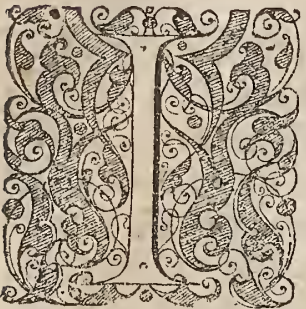

L fe fait encores d'vne autre forte de tuyaux nommées ordinaire. ment tuyaux acheminée, lefquels font vn peu plus forts de fon, que tuyaux bouchez, mais au refte ils ont la mefme harmoniela forme d'vn diceux eft icy fuiuant, \& la mefure des Siftefmes defdits tuyaux ne fe peut pas donner fi iuftes, comme les precedents, mais voici commelon procedera, lon prendra la longueur \& largeur du tuyau D. SOL RE. lequel eft vne tierce minor plus bas que F. FA, VT. \& ladite mefure feruira pour F. FA, VT. \& A A . Seruira pour C C . \& ainfi entre ces deux diftances l'on compofera toutes les autres lignes pour les autres tuyaux, \& pour la mefure de la cheminée, l'on prendra le quare de longueur de la circonference de chafcun tuyau pour faire la circonference defdites cheminees \& la moitié de la circonference de chafcun tuyau fera la longueur de ladite cheminće,

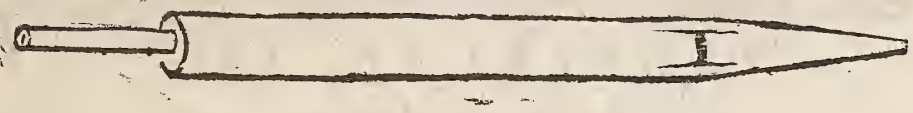

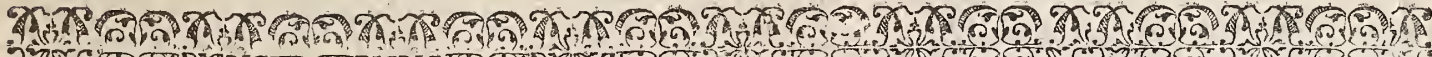

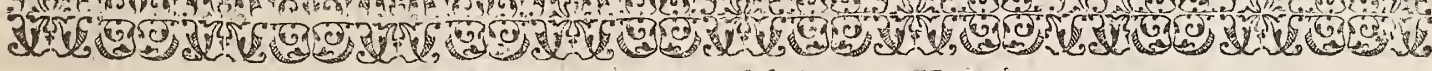
P R O B L E S E E V I I.

De la proportion de la bouche des tuy aux.

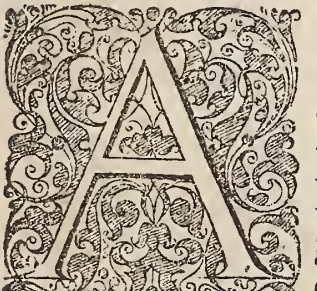

PRES que les tuyaux font taillez en la longueur \& largeur, il fera befoin de tailler la bouche, laquelle fe fera fumant la force quelon defre que les ruyaux fonnent, mais la façon la meilleure $\epsilon$ ft de di. uifer la largeur du tuyau en 4 . parties \& vne d'icelle metre au mil. lieu de la largeur du tuyau comme il fe peut voir aux fuiuantes figures, A.B.C.D. ou B.C. eft le quart de A.D. \& quand c’eft pourvn tuyaubouchéladitelargeur B.C. Re diuifera en quatre parties pour faire la largeur de louuerture B.E. F. C. mais quand ceft pour des tuyaux ouuerts ladite largeur de l'ouuerture, fe diuifera en s. parties \& vne dicelle fera a largeur de l'ou uerture, \& fi l'on veut faire fonner lefdits tuyaux plus haut il faudra faire ladite ouuerture plus large.

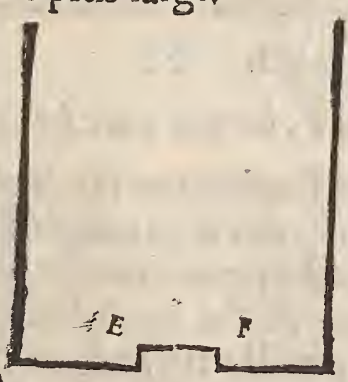

A

B C

L'ounerture pourles tuyaux outuerts.

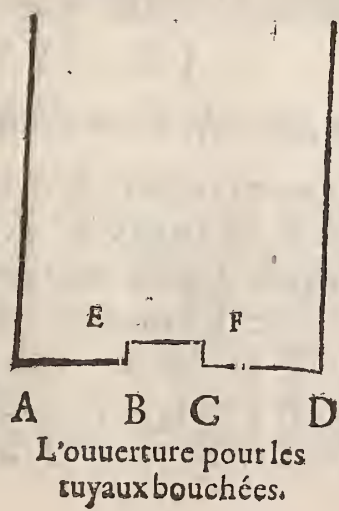




\section{Liure troifiefme.}

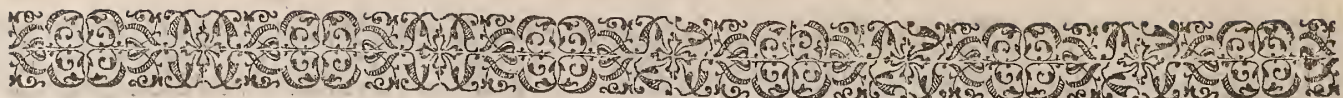

PROBLESMES. VIII.

\section{De la proportion de la languette des twy aux.}

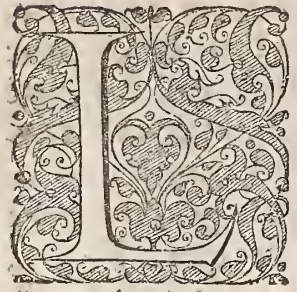

A languette des tuyaux eft vne platinelaquelle fe foude entrele pied du tuyau \& le corps \& léfpeffeur deladite platine fe fera de la troifiefme partie dela largeur de la bouche du tuyau, \& fe taillera en lendroit de louuerture vn peu én tallus pardehors affauoir le quart d'vn angle droit, \& fera bon dauoir vn petit triangle de cuiure pour voir que la taillede ladite languette foit au plus pres fuiuant icelle, \& faut aufi que ladite raille foit nettement faite, \& ferabon que le plomb dequoy fonctaite lefdites languettes, foit meflé d'vn peud'eftain f̧̧au oir deffus vingt liures de plöb $5_{\text {, }}$ liure d'eftain pour les rendre vn peu plus fermes, \& lon fera vne table dudic plomb a propos pour c'eft effect affez efpaiffe, \& l'on paffera les pieces par dedans l'inftrument demonftré auz, problefme felon l'efpeffeurque l'on les veut auoir.

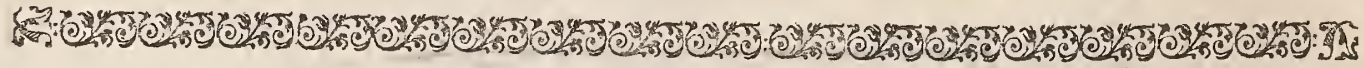

$$
\begin{aligned}
& \text { P R O B LES M E I X. } \\
& \text { Pour faire lepied des twy aux. }
\end{aligned}
$$

E pied destuyaux,tantaux ounerts comme aux bouchés fe taillera auec

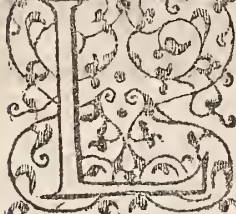
le corps du cuyau toure d'vne piece, \& deuant que le couper \& feparerledit piedd'auecle corps, l'on marquera auecla pointe d'vn cou. fteau les mefures dela bouche, commeil re peut voir à la figure fuiuan. te, \& auffil'on taillera le pied en cone comme il fe peut voir en ladite fi_ gure, \& 2 l'ounerrure par ou doibt entrer le vent, fe fera affez perite car apres que les tuyaux font pofés fur le fommier, s'yls n'ont affez de vent par ladite ouuerture elle fe pourra agrandirfacillement.

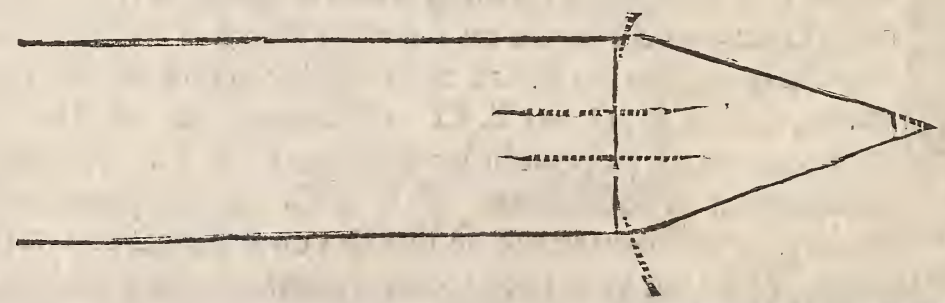

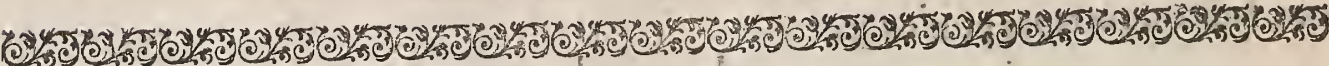
P R O B L E S M E X.

Pour accorder les twy aux d'orgues, les vns anec les autres.

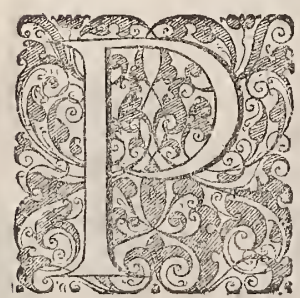

REMIER EMENT faut auoir le fondement fur le plus grand túyau du Siftefme qui eft F. FA, uT. \& s'il y a nombrede regiftrelon acordera celuy de trois pieds bouché premi erement, \& ainfi lon acordera toutes les notes qui fonten $\mathrm{F}_{+} \mathrm{FA}_{\mathrm{A}}$ ut. dudit regiftres ce qui fera aifé a faire prenant garde que les tuyaux ne fonnent point plus fore l'vn que lautre, \& que quand on fonne auec deux tuyaux enfemble a vne octaue, l'vin de lautre quill femble qu'il 
niena qu'vn, \& pour hauffer ou baiffer le fon des tuyaux bouchez, l.on foudera des petites platin es de plomb dites oreilles des deux cofte $z$ de la bouche defdits tuyaux, \& ferrantlefdites oreilles letuyau fonnera plus bas, \& les eflargiffant, il fonnera plus haut apres auoir acordéles F. FA. uT. il faudra acorderlesquintes defdits F. $F_{A}$ uт. qui foncles c. SOL. FA, uT. \& prendrecoufiours bien garde qu'vn tuyaune fonne point plus que l'autre, carc'eft vn grand defaut aux orgues quand aucuns des tuyaux fonnent plus fort les vns queles autres, apres l'on acordera les G. sol. R. ur. qui font vne quintechafcun plushauts queles C. sol. FA. uT. \& ainfi de quinte en quintelon acordera tout vn regiftre, mais il faut bien garder de ne faire lefdites quintes trop hautes ce qui arriue fouuent faute d'experience, \& apres qu'vn regiftre fera bien d'accord, il fera fort facle d'acorder tous les autres par octaues plus hauts, ou plus bas ouurans les bouts de haut defdirs tuyaux pour rendrele fon plus aigu, ou le reployant en dedans pourlerendreplus graue.

\section{5. PR O B L E S M E X I.}

De la conuenance quiont les rogistres les wns auec les autres.

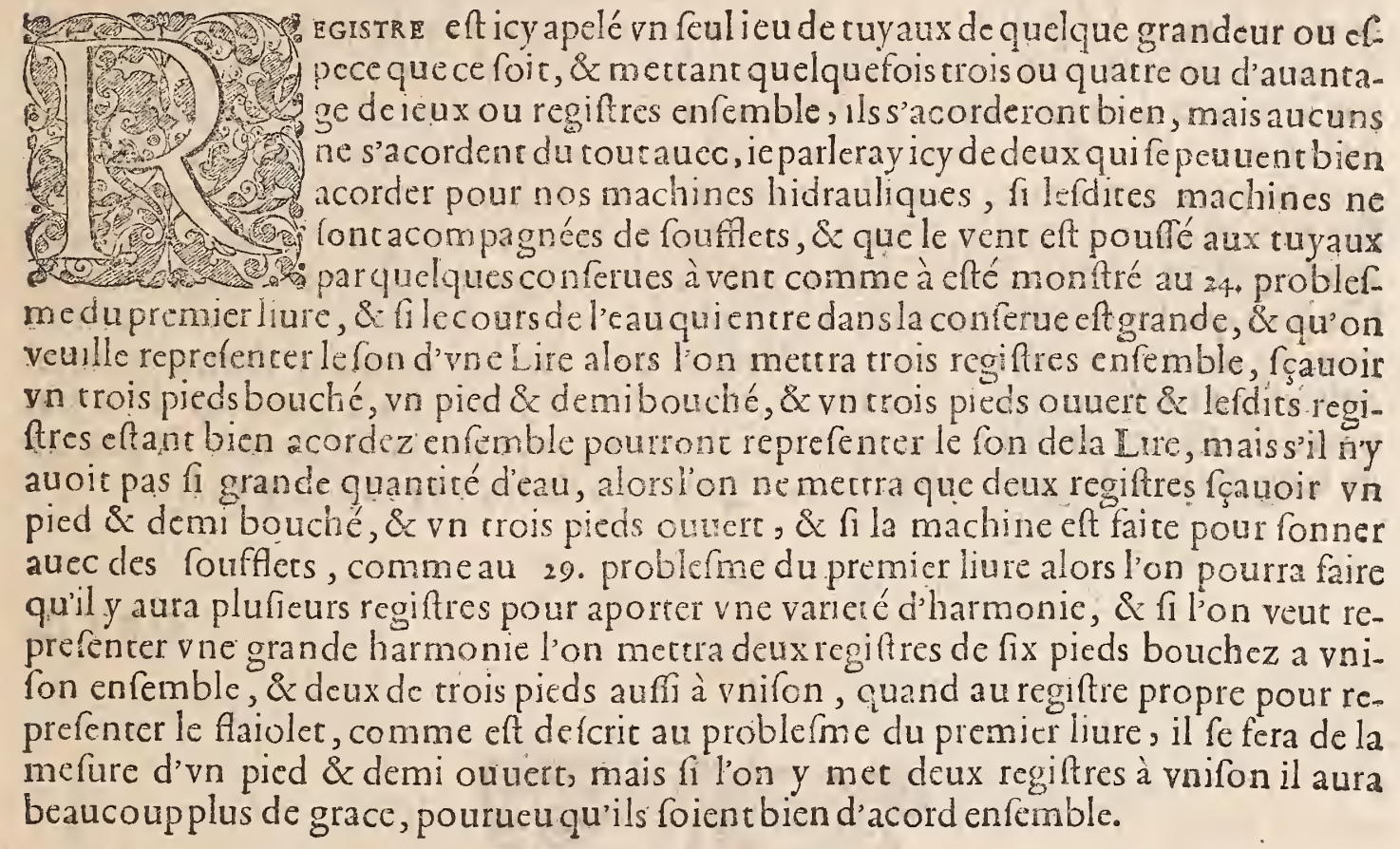

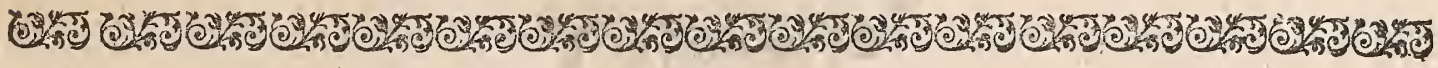

P R O B L E S M E X II.

Des Pedalles.

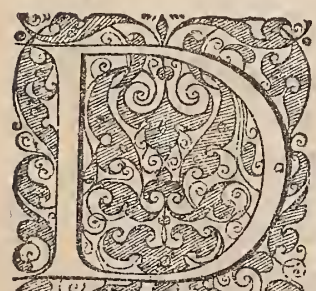

EPvis quelque temps en çàl'on a commencé à vfer de pedalles aux orgues, qui font tuyaux au deffoubs de F. FA uT. pour fonner vne octaue plus bas que les plus baffes comprifes au clauier, \& font apelées pedalles à caure que l'on ioüc du pied furleclauier defdits tuyaux i’en ay veu ou ily en auoit douzè̀ fçauoir C.D.E.F.æ. . G.A.B. P.C. D. E. autres n'en on tque troisà fçauoir C. D. E. les mefu. 6. 


\section{Liure troifiefme.}

\section{PROBLES M E XIII.}

\section{Du Sommier.}

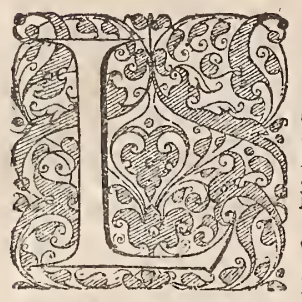

Efommier, d'aucuns apeléfecret, eft ce que Vitruue nöme canon mu. fical, lecjuel eft fait comme vn coffre ou eft enferré le vent qui vient des foufflets, \& dedans iceluy fontles foupapes, léquelles quand el. les font pouffées ouuertes, levent vient aux tuyaux \& les fait fonner, il faut que ledit fommier foit fait de bois de chefne fort fec $\&$ bien de droit fil, dont la partie de la figure fera ici reprefentée au plan perA. B.C.D. \& pareillement aux deux de lortografie, \& ingnografie, les pieces marquées $M$. fonc les foupapes lefquelles feront bien doublees de cuir bien doux \& vni à celle fin que quand elles font ferrez le vent ne paffe entre deux, la piece en l'ortografie marquée $H$. eft vn des refors de cuiure qui cien. nent lefdits foupapes ferrées, ce qui eft notté de la lettre E: en lingnografie font les graueures qui iont pourrées onuertes, ce qui eft noté de la letrre L. font les touches du clauier, lefquelles quand elles font abaiffées pas la force des doigts ou des cheuilles pofées en la rouë mufiqualle des machines hidrauliques, ouurent les foupapes, par le moyen d'vn perit fil de cuiure, qui les abaiffe, ce qui eft marqué en l'ortografie de la lettre F. eft vn des trous rond qui porte le vent depuis la graueure $E_{\text {* iuf- }}$ ques au porteuents marqués de la lettre G. \& eft befoing que ledit fommier foit fair auec grande diligence, \& que les foupapes foyent colées par vne queuë de cuir qui furpaffera le bout d'icelle en forte quelle puiffe ouurir \& ferrer bien iuftement $8 x$ fera bon que les refors de cuiure $H$. foyent forts aux fommiers qui doiuent feruir pour les machines hidrauliques, car eftans foibles comme ceux.des fommiers ordinairesil y a toufiours quelque chofe à raccommoder, quand à la grandeur de la graueure elle fera au moins demy pouce de large vn pouce de profondeur \& fix pouces de long, \& pour les gros tuyaux lefdites graueures feront vn peu plus grandes.

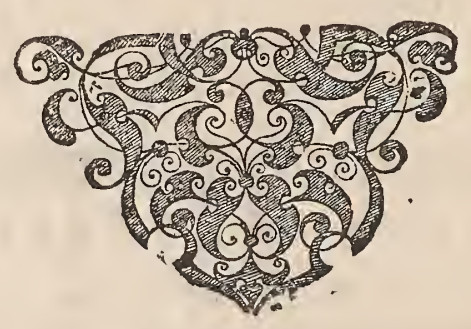




\section{Traitant de la Fabrique des Orgues.}
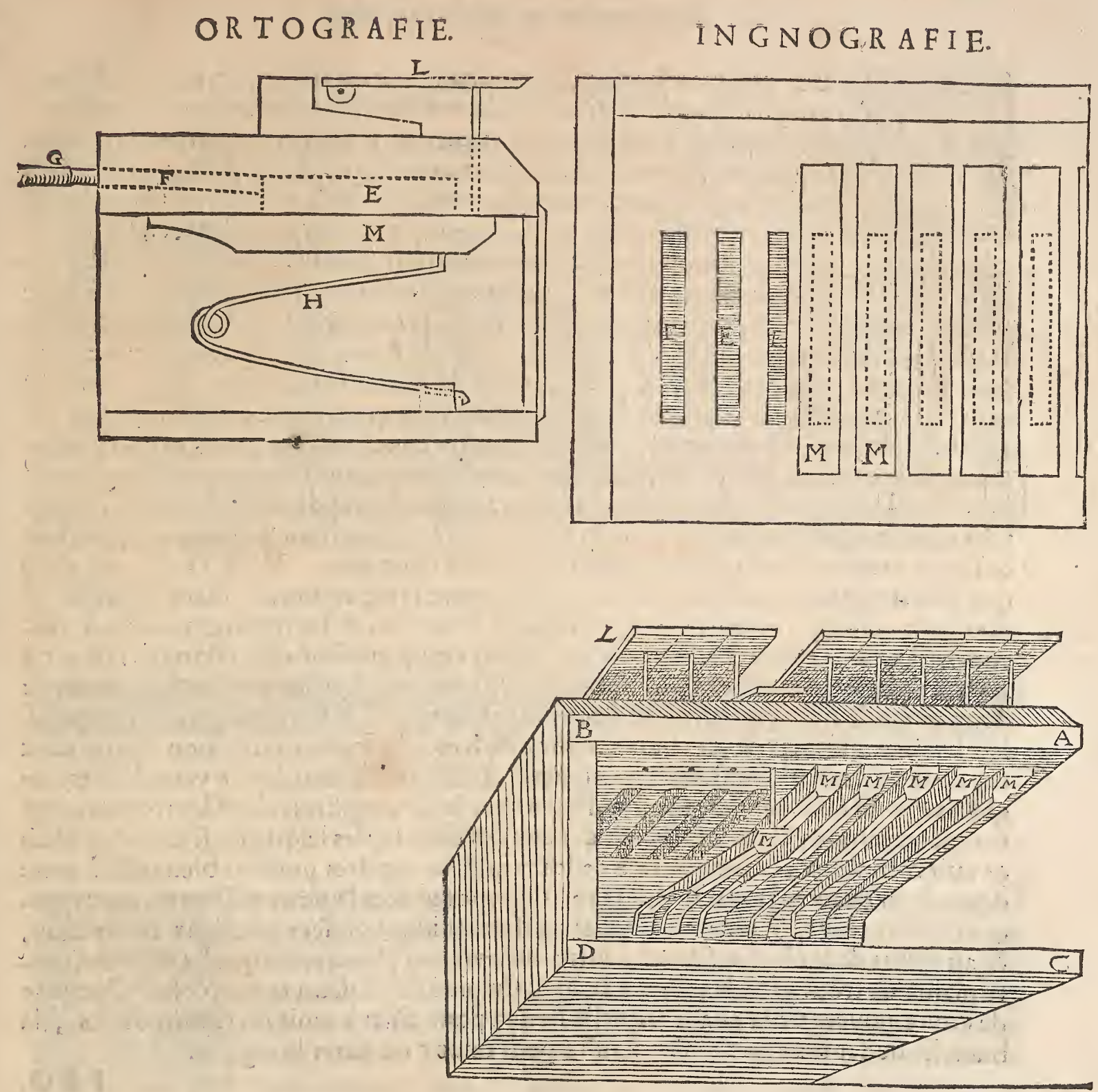


\section{Liure troifiefme,}

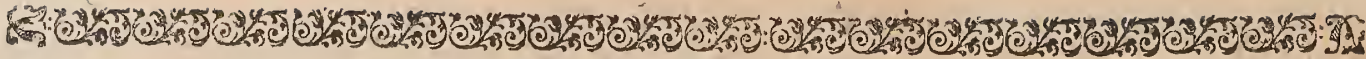
PRO B LE S M E X I V.

\section{Du fommier ou font les.regiftres.}

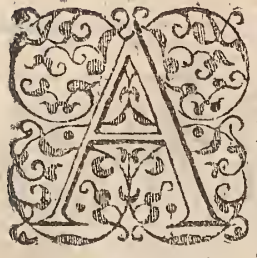

Ux orgues ordinaires, les tuyaux femettent fur le fommier, c'eft pourquoy il eft apelé ainfi, d'autant qu'il fouftient lefdits tuyaux, mais aux machines hidrauliques il fera bon que le fommier foit diuifé en deux, fçauoir vn, auquel fera les foupapes duquel à efté parlé au precedene problefme, \& l'autre duquel noưs parlerons à prefent, ou feront les rey puiffe auoir vne muraille ou quelgue feparation entre lefdits fommiers, à celle fin de noüir le bruit du mouuement de la machine, \& le vént fera communiqué à ce prefent fommier de l'autre, par des porteuents, la grandeur dudit fommier fera felon la groffeur des suyaux que lon à de poffer deflus, la forme d'iceluy fera comme la figure fuiuante marqquée A.B.C.D. la table de:deffoubs marquée I. L. fera de bois de chefne bien fec, de deux pouces d'efpais, \&fera percée de cofté (auec vne tarelle bien droite) à vn pouce pres du bout, fçauoir en ceftuy-ciêz. trous, \&rsil y a d'a. vantage de touches, l'ony mettra d'auantage de trous, puis l'on mettra des reigles de bois bien droites autant comme lon voudra auoir defortes de ieux', lefquelles reigles font apelées regiftres marqueés E. F. G. H. alants d'vn bout à lautre du fommier \& feront areftées àdes petites chenillettes de fer marquées M. N. O.P. en forte que lefdits regiftres puiffent gliffer entre vne autre table nommée chape laquelle eft marquée R. Q. apres lon percera la rable de deflus, \& les regiftres iufques à ren. contrer les trous trauerfans, en forte que lefdits trous puiffent eftre eflongnés de 3, ou 4. pouces felon la groffeur des tuyaux, \& fera bon que chafcun regiftre foit percé de deux rengs de trous, comme il fe peut voir en la figure, \& faut que quand l'on pouf. fera lefdits regiftres, queles trous qui font en iceux, re rencontrent, non contre ceux des deux tables, | mais iuftement en tredeux; à celle fin de boucher le vent defdits regiftres quand l'on voudra, \& quandl'on tirera lefdits regiftres, alors les trous d.iceux 帒 rencontreront viftement vis ausis deceux des deux tables lefquelles feront dou blées de cuir bien doux, \& bien colé à celle fin que les regiftres puiffent bien gliffer entre deux, \& en outre faut que lefdites tables foyent fermées l'vne auec l'autre, auec quelques vis ou dous, en forte que lefdits regiftres puiffent gliffer entre deux facillement, $\&$ au deffus de la chappe fera vne autre table vn peu plusefpaiffe que les regiftres, toute plaine de trous grands comme le haut du pied de chafcun tuyau, \& fera eflongnée de 6. oư 8. pouces de la chape laquelle feruira pour aider à tenir les tuyaus droits, $\mathrm{I}_{2}$ branche de fer marquéé T. feruira pour outxir ou ferrer le regiftre.

PRO.

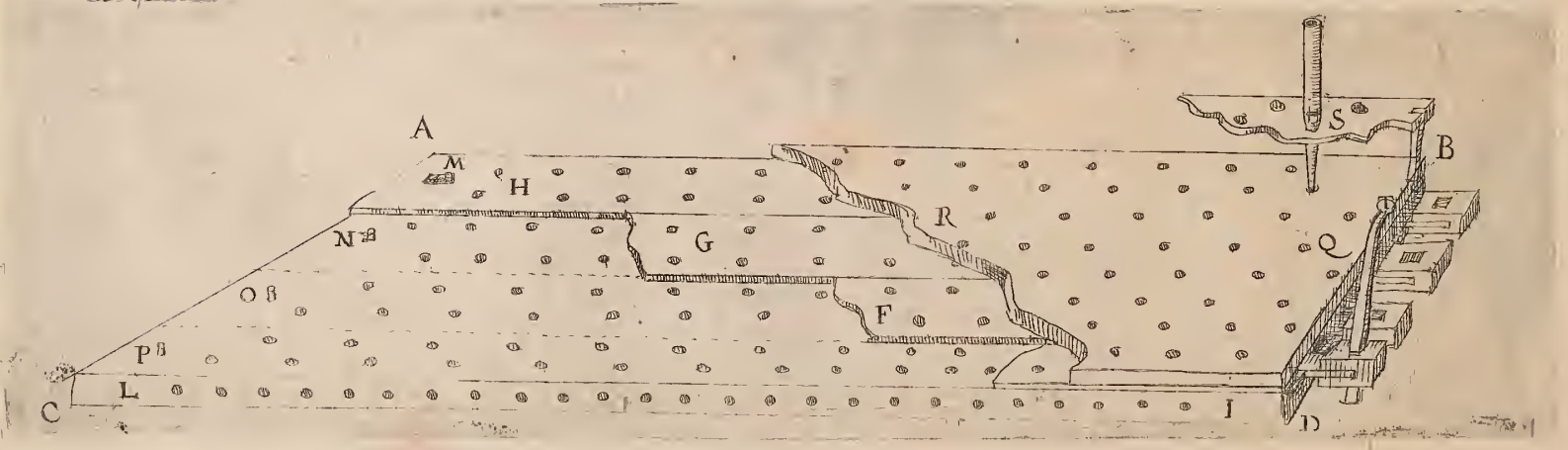




\section{Traitant de la Fabrique des Orgues.}

\section{PROBLES ME XV.}

Desparteuents.

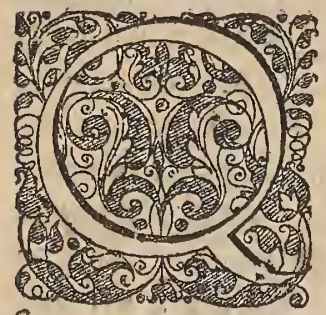

VAND aux porteuents ilsfe feront de plomb ou de cuiure, \& sil y a s. ou 6. pieds de diftance entre les deux. fommiers, \& qu'il y aye 3. ou 4. regiftres pour fonner enfemble, on fera lefdits porteuents d'vn pouce en diamettre par dedans \& feront bien ioints, dans les trous defdits fommiers, \& s'il y a des tuyaux qui furpaffent 3 . pieds à ceux la on les fera plus grands, il eft bien vray qu'il y a fort peu d'orgues, ou les porteuents foyent fi grands, mais auffi c'eft vne faute ordinaire de les auoir fi petits, \& cela eft caufe quill faut vn grand pois fur les foufflets, \& auffi les tuyaux n'en fonnent pas fi nettement, \& s'il fe peut faire que les foupapes foyent fort grandes/, \& generallement toutes les graueures \& conduits pour conduire le vent aux tuyaux, car par ce moyen les foufflets nauront que, faire deftre fi forts chargez de pois, \& auffi les tuyaux aurone le fon beaucoup plus net, \& a ceux ou le vent viendra trop fort il faudra ferrer le bout du cuyau autant quil faue pour le faire fonner en fa nature.

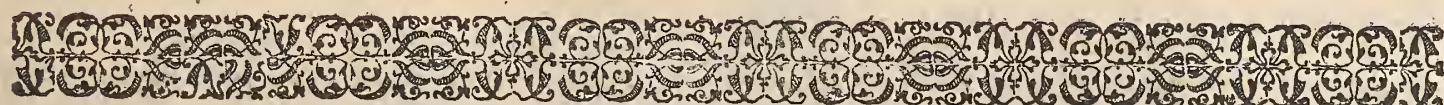

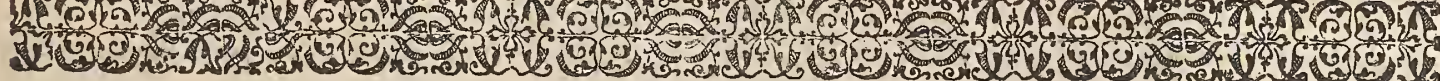

PROBLESME XVI.

$$
\text { Des fouffets. }
$$

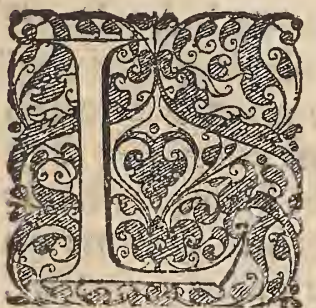

Es fouffets feront felon la proportion des tuyaux \& regiftes, sil y a trois ou quatre regiftres, \& que le plus grand tuyau ne foit que de trois pieds, les foufflets auront au moins cinq pieds de long \&e deux \& demi de large, \& feront au nombre de 4 . fi ceft qu'ils doiuent eftre leuez par la force de l'eau comme à efté monftré au 29. problefme du premier liure, autrement fi on les léue auecla for. giftres, \& auffi qu'il y aye de plus grands tuyaux, l'on fera les foufflets plus grands \& en plus grand nombre, car le plus qu'il y en a, fera le meilleur, \& aufif le plus pres qu'ils feront du fommier, \& les faut faire en forte qu'ils puiffent tenir bien le vent, en doublant bien toutes les fueilles de bois par dedans, \& auffi le cuir qui ioine lefdites pieces enfemble de parchemin bien collé, car le vent paffe tant à trauers le bois comme auffi du cuir sil n’eft doublé de la façon. 


\section{Liure troifiefme,}

10.6. ron P R O B L E S M E X.VII.

\section{Du tremblant.}

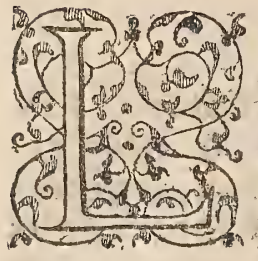

E tremblanteft vne petite feneftre, laquelle eft dans le porteuent entre les foufflets \& le fommier, \& eftant abaifféc caufe le vent a fortir par fauts, qui fait faire vn tremblementaux tuyaux fort agreablea loüie, 12 figure dudit tremblant eft icy reprefentée par la lettre: A. \& au deffus du porteuent, il y aura vn trou quarré marqué B. \& par iceluy lon pourra mettre autant de pois qu'il fera conuenable pour faire le-

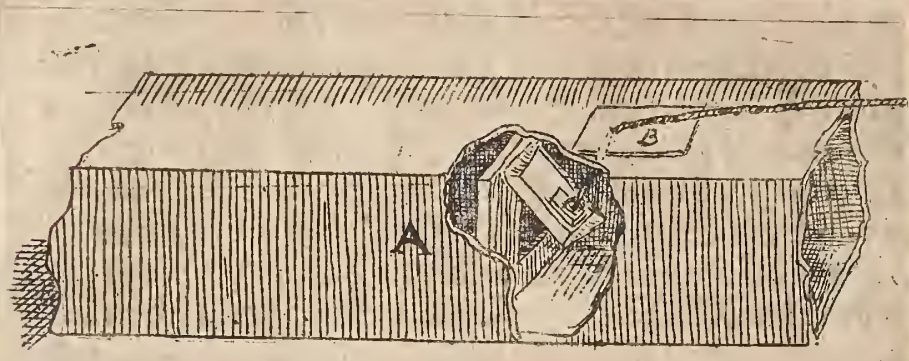
dittremblant trembler à propos, \& fuiuant la grandeur \& pefanteur qu'ily a fur les foufflett le porteuent fera grand fçauoir sils ont quatre pieds de long \& deux de!large, ledit tramblant aura demipied delong \& quatrepouces de large, \& s'ils ont fix pieds de long, \& rrois de large, il aura 8.pouces de long, $\&$ fix de large, \& faudra qu'il y aye vn petit anneau deffus pour ypaffer autant de platines de plomb, comme il fera befoing pour le faire trembler àpropos.

M. dU - Aucunes $\mathbb{R}$ cigles en general, poser la fabrigue des Hidrauliques.

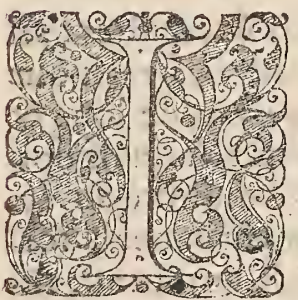

L y a plufreurs chofes remarquables en la fabrique deshidrauliques que la practique enfeigne, \& dont on fe trouueroit empefché d'en rendre raifon, quand lon veut faire fonner deux regiftres à vinifon l'vn de l'aurre ou a l'octaue fi lefdits regiftres font eflongnez l'vn de lautre de trois pieds ou enuiron, lls fonneront bien plus hauts enfemble, que sils eftoyent ioingnants l'vn de l'autre, comme on fait ordinairement, ceft pourquoy quand la placele peut permettre, il fautellongner lefdits regiftes aucunement l'vn de l'autre, \& ne faut pas auffi les mertre fil loings, car la longueur des porteuents empefche, \& eft caufe qua il faut vn plus gramd pois deffus les foufflets, \& fi la machine eft faite en forte que le vent vienne aux tuyaux, d'vne conferue à vent, alors il fera: bon que les tuyaux foyent de cuive, \&fpecialement les petits, \& les grands auront les languettes, \& le pied de cuiure, ic refte pourra eftre de plomb, \& ced'autant quel'air qui procede de la conferue, eftextremement humide, \& eftcau fe de gafter le plomb \& l'eftain \& y engendrer de la cerufe qui bouche quelquefois l'ouucrure de la bouche, \&le pied des tuyaux, ce quiles empefche de fonner, quand aux foupapes des machines qui iouënt auec les foufflets, elles feront larges au moins d'vn pouce, \& fix ou fept de long, qui eft plus qu'ordinairement on ne donne aux orgues de moyenne grandeur, mais aux ordinaires, le clauier eftabarfé fort bas, ce quicaufe les foupapes de s'ourir fort larges, mais aux hidrauliques, quand 


\section{Traitant de la Fabrique des Orgues.}

ce font des crochets, ou demis crochets, (à caufe de la viteffe du mouuement) lefdites foupapes ne fe peuuent pas beaucoup ouurir, c'eft la raifon pourquoy il les faudra faire vn peu plus grandes, a celle fin d'auoir la graueure plus large, il y a auffivne chofe fort a confiderer, c'eft que fil'on defire fe feruir de fouffets, dedans quelquegrote, il faut que le licu ou ils feront, foit forr fec \& non humide, comme aufi le refte du mouuement, \& en oultre, il fera bon que lefdits fouffers foient enclos dans vne perite chambrette bien clofede planches, y laiffans feulement vne peciteouuerture, pourlaif fer entrer l'air dedans pour lefdits foufflers, \& fi la place eft fort humide, alors l'on fera la machine auec des robinets \& vne conferue a vent, commeàeftéenfeigné en la fin du premier liure, laquelle inuencion eft plus rare, \& exquife que l'autre, mais aufi elle eft plus difficile, \& eftant vne fo is bien faite elle peut eftre de longue durée \& aporter vn grand plaifir, ic mettray fin pour le prefent à ce troifiefme liure efperant auecle temps d'en faire encores vn ou feront monftrées quelques machines fort rares, \& que ie tiens fort fecrettes, \& entre les autres, vnequi reprefentera vre mufique plus parfaictequaucune humaine creature ne peut faire, foitauecles voix ou inftruments $m$ anuels 


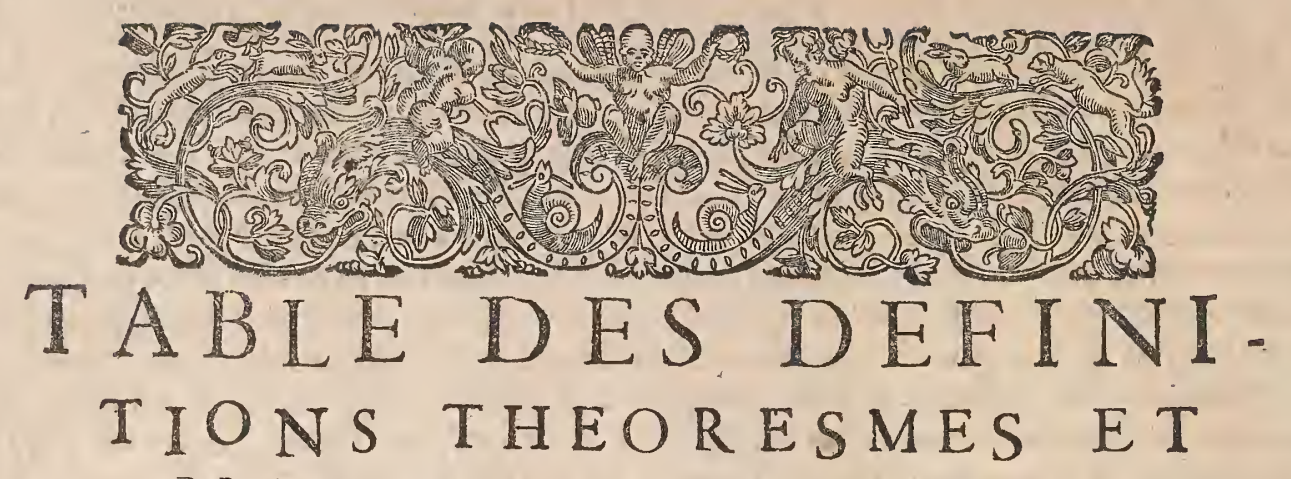

\section{PROBLES MES CONTENVS AVX} trois precedents liures.

\section{DEFINITIONS.}

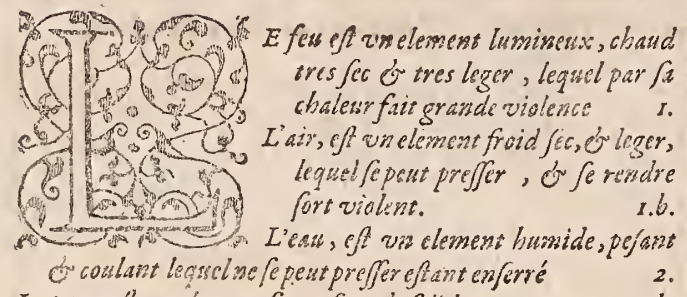

Laterre, eft wrselement fec, pefaint é Solid:

\section{THEORESMES.}

I espariesdes eloments femeng nt enfemble pour in semps, L puischafcurn diceuteretousne en fon licu.

Ilniy a rien a nouscogneu de vaide.

Autre devnonfration couchant le vuide.

Quandleau monte par faute de vacuité, cefte pour difcendire plusbas que fon minesu.

L'eau ne peut monflerpar fos propre moyon. ficen'eftpour des. ccridre plus bes que fon niucau.

L'eatw montera par aidedu feu plus baut que fon niwean.

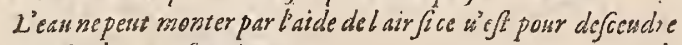
plus bas que fon niueaus.

Domonftration de la bautcur que la wachine de Heronepessi fairemonier leatu.

L'ean peue monter en baut par diuer fes machines cosnduites par fa force mefme, out astre que ce foit

Aux machines proprespeurl'eaus, lapefanteur de ladite eau 5 e mefureparfahauteur.

L'air paffè trawers lear quand il eft preffé.

La force ducontrepois qui fait mounoir vne balanceeft proportionsee fuivant foreflangrement du point de grauité. s.6.

si run des bouts de la furddite balance ou fleaus eft abaijsé lautre fe Lenera e toucesles findites parties mouneront, en proportion dupoint de grauitê.

Le temps de la motion sacorde auec le mounement du contre-

pois
Lemounemert du levier s'acorde auec celuy de la balance
6.6 .6 . Aux macbinesquiffor fort ativer fardeaux par le moyen despoulies,filaforce eft double, Ton tivera 20. pieds de corde, pour faire lester le far deau yo. pieds.

Aux roue deratelees, Si vn pignon fait s. tours, pour faire mousnoir vne roue dentelee vn tour ó que laxe de ladite roue foit en diamettre comme ledit pignon, ladite axe lewern s. fois antant queledit pignon
Parla maltipliontion de la force, on leucra vn fardeas quelpoCant quillfoit.

De la force du piznon a vis.

7.0.

La force de hi geffea vis. 80 8.6 .

\section{- PR O B L E S M E S.}

Pourfaire fluer beaup pr le courane d'vne riwiere, ó ho form Autremoyen de leuer leau par le moyen divn ruiffeau: Pour efeuer vne eau de fource ou de riwiere par la force des sheHaux.

Plan de l'orrographie de la precedente macbine 13. Pour faire elescr partie de l'eas d' one fource, cing ouf fox pied's baut Machine fort fabtillepar lequelle les vayßeaux de la precedess. ce s'ourent of ferreut de cux mejmes, par le moyen de teat. is.

Pour faire vnorloge auec le cours d" vne fonsaine naturelle th-

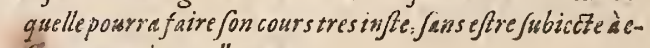
fire montee iourssellement. 15. Autre maniere d'orologe d'eau. 17. Powr faire vn waifferu, anquel metzant do leau par force, or ortirs pris apres auec grande violence is Pour contrefaire la voix des perits oifeaux par le moyen de leau d lair.

is.

Pour adioufter aufindit moustement vn cigas, ou quelave autre oifeau, lequel botra autant deaucomme on luy donners is Pour faire vne machine, laquelle aura mounenoens de foy mefme Machine fort fubtille par laquelle on pourra faire efeuer vne caudormante. 20. Machine par laguelle lon pourra augmenter la force de la precedente

$2 r$.

Maniere defaire le ciment pour cimenter les verres aux vaiffeaux, en forte que lair n'en puiffe fortir $\quad 2 R$ Autre maniere pour augmenter la force de la fontaine presedente

22

Pour faire monter licau par le mayen despompes of d'vne roue à eart.

Orthographie de laprecedentemachine. $\quad 24$

Machine par laquelle l'on pourra par la force d'vne roue àeau, faire fier du bois, auee grande promptitude is crachine de grand feruice, propre pour pereer des pipesde bois.

Machind 


\section{Table.}

Macbine fort wecefsire par laquelle lon pert donner grond fecours aux maifons qui feroyent enflambees.

Macbine fort fubtile pour tourner en oualle guelque chofe que Se foit.

Pour faire reprefenter le chant d' us oifean en fon natsurel, par le moyen de leau.

Pour faire reprefenter plufeurs difeaux le guels chanteron diuer fement quand vnechouette fe tournera vers iceux, \& quandladice chouette fe recournera, ils cefferont de chanter. $3 T$.

Machine par laquelle lon reprefentera one Galatee qui fera trainee fur l'eaw par deux Daufins, allansen ligne droite, of fe retournant d'elle mefme cependani quivn Ciclope ione deffus on flaiolet.

Machine par laquelli lon reprefentera le fon d' wn fuiolet auec le cours del eau.

Plan Ingnografique dé la grotede la Galatee deforipte au ving. troifiefme proble ime, or celuy aufie, pour faire vouer le flaiol. let defirit au 2 ax. problefme
Cuachisepar laquelle fer a reprefenté von Nepiune, lequel tournera circulairement, a lentour dive roche, auec quelques austres figures, le Guelles ictteront de leain en tournant. 36 Machine par laquellel'on fera fonner un ieu d'orgues, parle moyen de l'eau.

Machine par laquelle les fouffets de la precedente, fe pourront baufer pour conner le vent aux tuy.zux d orgucs $3 s$ Reprefcntation de la rouc mufiqualle, enplus grandeforme, pour fercuir au proble fine 28 . 39. Machine hidraulique, par laquelle dés orgues pourront fonnier anec leau fans aide de fouffets.

Autre defeing de la precedente machine.

Plan Ingnografique, de ls precedintemachine. Commeilfaut construire la conferue a vent pour les machines hidrauliques.

Pour faive vue machine admirable, laguelle eftanipofee as pied d'vnefigure, icttera unfon wis lesser du Solvil. of griatd le Soleildonnera deffus, en forte giu il femibler a que laditefigure face ledit for.

\section{2.}

\section{T A B LE DV SECOND LIVRE.}

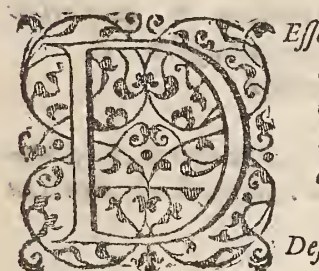

delear.

Deffeing de la font aine du cupidon, ou il y arra vine tourterelle guiboira autant d'eau, comme on luy donnera.

Deffeing d'vne font aine d'ordrerufigue

Defeiug d vane voliere a oifeasu auec quelques grotes de 4. Autre deffeing d vne autre voliere a oifeauxplus grande, acompagneed'vn parillon au millieu.

Plan perpectif duprecedent deffeing.

Defjeing dvine montaigne au milliew dvn iardin auec quelques grotes dedans.

TO.

Deffiing d vno haute terraffe acompagnee de quelques grotcs pour inetre dans vniardin.

Defeing daf frontipice de la grote fatnee forr la terraffe duptrece dent deffing 12.

Diffeing d'vn mont parnafe, ou lon pourr a fuire quelques groresdedans

Deffeing drue figure grande reprefentante le mont Tmolises. 14.

Deffeing de la grote de Tmollus. Is.

Defferng d voue grande figure reprefintant vn fleure 16. Deffing d'one grote d'Orfee quife pourra faire dans la figure precederste.

Deffeing d" rne zimphe qui ione des orgues à laquelle on $E f$ : chorepond.

Deffing drinc fontaine propre pour on Iardin

Pour la condute des eaux de fortaines. 20.

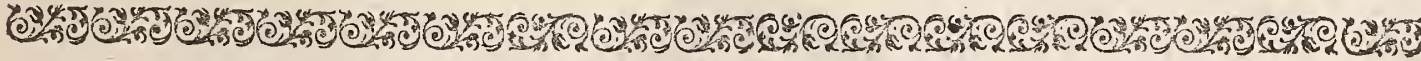

\section{TABLE DV TROISIESME LIVRE.}

$\mathrm{D}$ IF linuention des machines bidrauliques Ce qui eft requis pour la fabrique des orgues Comme il faut ietter le plomb of estain pour la fabrique des orgues.

Infrument pour faire le plomb fort wny. Comme il faut donner la me fure au Diapafon Pour donner la mejure aux Siftefmes bouchees Pour faire les Sifte mes de ruyaux ounerts pour faire les Siftefmes de tuyaux a cheminee De la proportion de la bouche destuyaux De laproportios de la languette destuyaux.

\section{f.r. | Pour faire lepied des tuyaux} Pour acorder lestuyaux d'orgues les ins auec les autres 4.6 De la conuenance des regiftres les uns aue les autres S. Des Pedalles

2.6. Du Sommier

3. Du fommier ou font les regiftres

3.6 Desportenerts

3.6 Desfouffets

4. Du tremblant 7.6

4. Lucunes reiglesengener al pour la fabrigue des bidrauliques 7.6 gesengeneral pour lafabrique des hidrauligues

FIN. 


\section{Faütes à corriger.}

Sueillet 1. ligne is. car rowt antre fen ou chaleur, eft fubiecte a nourriture, \& ce qui eft fubsect à perir.

Fueillet 3. ligne 2. qu'ilfepeut voir que l'eau s'estoit efuaporee. Fueillet 3. b. ligme 8. pour laiffer paffer entre ledit bout Fueillet $\sigma$. ligne 18. \& foit le milieu de ladite ligne, le point de grauitémarqué $C$.

ligne 28. conrepondant

Fueillet 9. ligne 4. Joupages

Fueillet 12. b. ligne 22. bareils

Fueillet 14.6. ligne 27. quand à la recourbeure du tuyas

Fueillet 18. b. ligneg. de faire des ousres perpetwelles Le mefme, ligne is. cefte difpofition naturelle Auproblefme 26.ily doit auoir

Fueillet 39.6.trenciefme robinet

Fueillet 43. 6.ligne 27. pones en dismettre
Lifez car tout autre few on chalesur of fubiecte à nourriture occe qui eft fubiect à nourrisure eft fubiect à perir.

Lifez qu'ulfepeut voir que leaw qui s'eftois efusporee.

Lilez pour laiffer paffer l' ean entre ledit bout.

Cela eft imprimé deux fois of le faut lire jewlement whe

Lifez correpondant.

Lilez foupape.

Lifez barils

Lifez quand a larecourbeure du tuyas $K$. elle ef faite pour empelcher quel'eaus du vaiffeaw $A$. quand il est" plain, ne iombe par iceluy tuyass au vaiffesu $B$.

Lifez diffairedesaunesperpetuelles

I ifez ceftediproportion

Plan ingrogr afigue de la grote de la 3 alatee def crite an 24. proble ine, \& celuy aufjpour faircioner leflaiolet deforit an 25. problefme.

Lifez troifiefmerobinet

Lifez poucesendiamertre

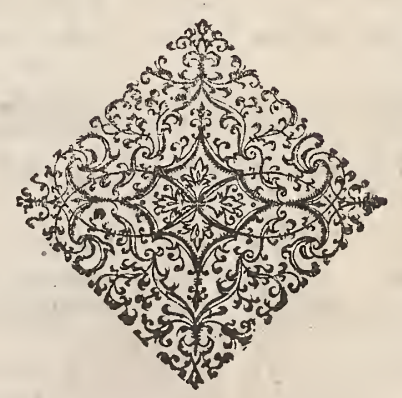

Ici fuit le Siftelme ou Diapafon defcrit au 3. problefme. 







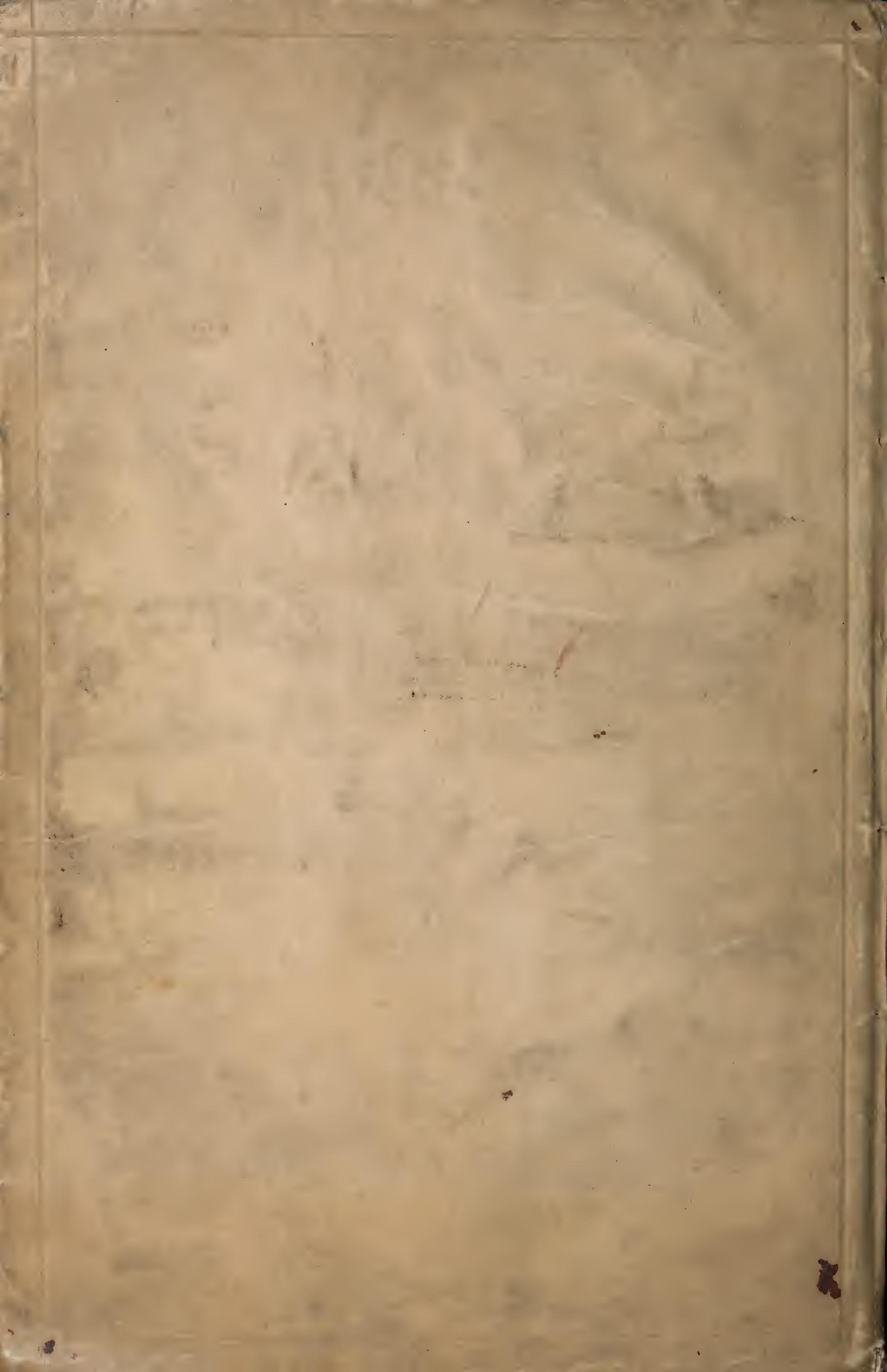

